\title{
Finsler Transnormal functions and singular foliations of codimension 1
}

Hengameh Raeisidehkordi

\author{
Thesis SubMitTeD \\ TO \\ INSTITUTE OF MATHEMATICS AND STATISTICS \\ $\mathrm{OF}$ \\ University OF SÃo PAUlo \\ FOR \\ THE DEGREE \\ $\mathrm{OF}$ \\ Doctor of Philosophy in Mathematics
}

Program: PhD in Mathematics

Advisor: Prof. Marcos M. Alexandrino

During the progress of this work the author received financial aid from CAPES and CNPq

São Paulo, March of 2018 


\section{Funções transnormais Finsleriana e folheações singulares de codimensão 1}

\footnotetext{
Esta versão da dissertação/tese contém as correções e alterações sugeridas pela Comissão Julgadora durante a defesa da versão original do trabalho, realizada em 09/03/2018. Uma cópia da versão original está disponível no Instituto de Matemática e Estatística da Universidade de São Paulo.
}

Comissão Julgadora:

- Prof. Dr. Marcos Martins Alexandrino da Silva (orientadora) - IME-USP

- Prof. Dr. Paolo Piccione - IME-USP

- Prof. Dr. Dirk Toben - UFSCar - Externo

- Prof. Dr. José Barbosa Gomes - UFJF - Externo

- Dr. Benigno Oliveira Alves - Externo 


\section{Acknowledgment}

I would like to dedicate my $\mathrm{PhD}$ thesis to my beloved husband, Dr Majid Forghani, for affectionately standing by my side, for his unwavering belief in me and his patience during those years. I can not thank you enough for your encouragement throughout this work. I also would like to thank professor Marcos Alexandrino for his counsel and advice. Your advice on both research as well as your support have been invaluable. A special thanks to all of the professors in IME who generously shared their knowledge with me and helped me as I was a foreign student with no knowledge of Portuguese. Words can not express how grateful I am to Professors Rosa Chaves, Pedro Salomão, Claudio Gorodski, Pedro Zühlke and all of the friends that helped me accomplish my PhD. 


\section{Resumo}

Raeisidehkordi, H. funções transnormais finsler e folheações singulares de codimensão 1. 2018. 123 f. Tese (Doutorado) - Instituto de Matemática e Estatística, Universidade de São Paulo, São Paulo, 2010.

As funções transnormais são a generalização da função de distância e este tópico tem algumas aplicações em Física e no mundo real. Neste trabalho, alguns resultados do caso riemanniana para o Finsler são generalizados. Alem disso, alguns fenômenos novos que ocorrem apenas nos espaços de Finsler são discutidos. Para ter uma melhor compreensão, são fornecidos certos exemplos com base nos resultados mencionados nos espaços de Randers. Além disso, algumas aplicações sobre propagação de ondas de fogo e água são introduzidas.

Palavras-chave: Funções transnormais, variedades Finsler, variedades Randers, foliação Finsler. 


\section{Abstract}

Raeisidehkordi, H. Finsler Transnormal functions and singular foliations of codimension 1. 2018. 126 pg. Thesis (PhD) - Institute of Mathematics and Statistics, University of São Paulo, São Paulo, 2018.

Transnormal functions are generalization of distance function and this topic has some applications in Physics and real world. In this work, some results are generalized from Riemannian case to the Finsler one. Moreover certain new phenomena that happen only in Finsler spaces are discussed. To have a better understanding, certain examples based on the mentioned results in Randers spaces are provided. Moreover, some applications on propagation of waves of fire and water are introduced.

Keywords: Transnormal functions, Finsler manifolds, Randers manifolds, Finsler foliation. 


\section{Contents}

$\begin{array}{ll}\text { Introduction } & 1\end{array}$

1 Preliminaries $\quad 5$

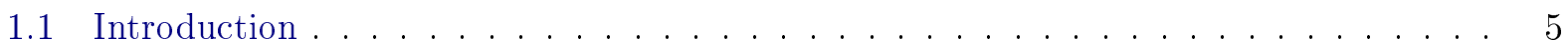

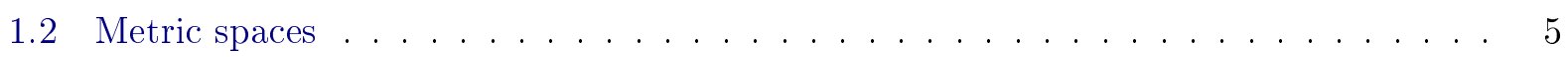

1.2 .1 Minkowski spaces . . . . . . . . . . . . . . . . 5

1.2 .2 Finsler Spaces . . . . . . . . . . . . . . . . . . . . 7

1.2 .3 Randers spaces . . . . . . . . . . . . . . . . . . . 9

1.2 .4 Length Structure . . . . . . . . . . . . . . . . . . . . . . . . . . . . . . . . . . . . .

1.3 Geometric Objects . . . . . . . . . . . . . . . . . . . . 11

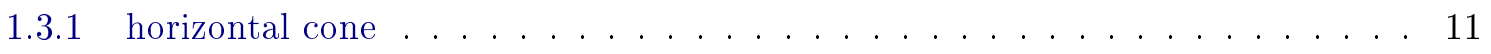

1.3 .2 Chern connection . . . . . . . . . . . . . . . . . . 12

1.3.3 Geodesic and exponential map . . . . . . . . . . . . . . . . . 14

1.3.4 Jacobi field and conjugate and focal points . . . . . . . . . . . . 15

1.3.5 Gradient and Transnormal Finsler functions . . . . . . . . . . . . . . . 19

1.3.6 Morse-Bott functions and Transnormality . . . . . . . . . . . . . 21

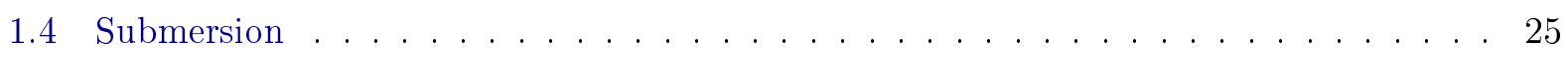

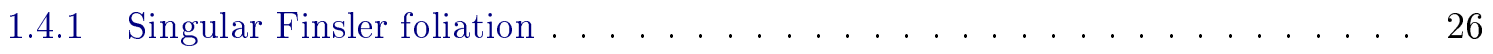

1.4 .2 Parallelism . . . . . . . . . . . . . . . . 27

1.4.3 Tubular neighborhoods and geometric cylinders . . . . . . . . . . . . . 29

1.5 The past metric $\tilde{F}(v)=F(-v)$ and whose geometric objects . . . . . . . . . 34

2 Randers spaces $\quad 39$

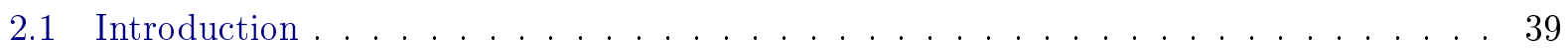

2.2 Zermelo navigation and Randers metric . . . . . . . . . . . . . . 39

2.2.1 The explicit expression of $F$ and some geometric objects . . . . . . . . . . . 40

2.2.2 Some results on Randers transnormality . . . . . . . . . . . . . . . 43

2.2 .3 Geodesics in Randers spaces . . . . . . . . . . . . . . . . . . . . . . 47

2.3 Randers-Minkowski space . . . . . . . . . . . . . . . . . . . . . . . . . . . . . . 47

2.4 On Finsler foliation in Randers spaces . . . . . . . . . . . . . . . . . . . . . 49

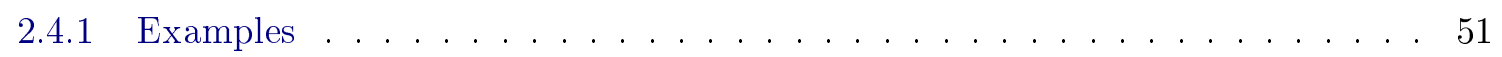

$\begin{array}{lll}3 & \text { Some results on Finsler foliations } & 67\end{array}$

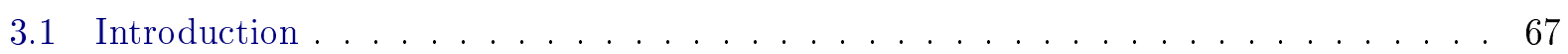

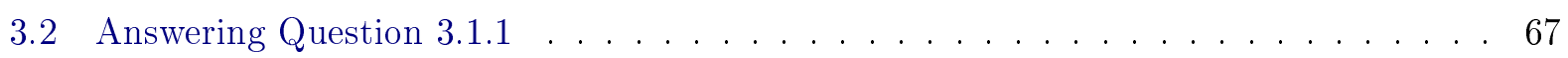




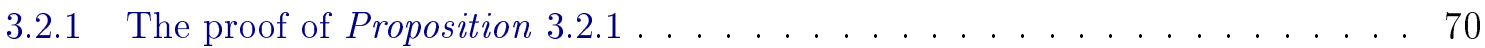

3.2 .2 Examples . . . . . . . . . . . . . . . . . . 73

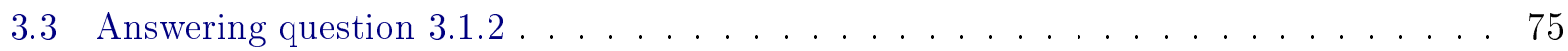

3.3 .1 Theorem and proof . . . . . . . . . . . . . . 75

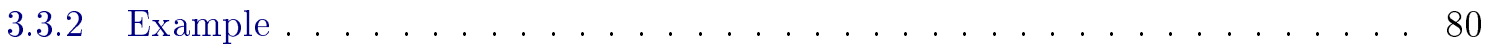

3.4 Answering Question $3.1 .3 \ldots \ldots \ldots \ldots \ldots \ldots \ldots$

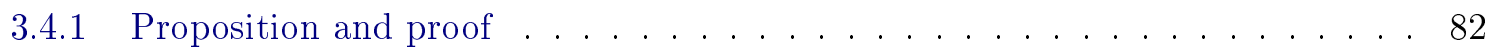

3.4 Examples . . . . . . . . . . . . . . . . . . . . 88

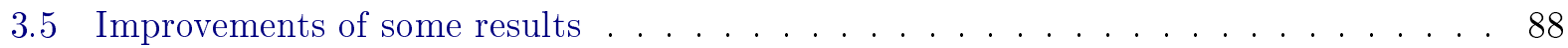

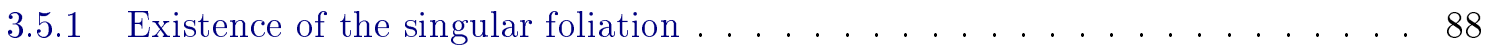

3.5.2 The distance between the leaves . . . . . . . . . . . . . . . . . . 92

4 Applications $\quad 101$

4.1 Introduction . . . . . . . . . . . . . . . . . . . 101

4.2 Huygens's principle . . . . . . . . . . . . . . . . . . . . . 101

4.2.1 The translation of Huygens' principle into the language of mathematics . . . 104

4.3 A mathematical modeling for wildfire . . . . . . . . . . . . . . . . . . . . 104

4.3 .1 A special case . . . . . . . . . . . . . . . . . . . . 108

4.4 the propagation of water waves $\ldots \ldots \ldots \ldots \ldots \ldots$

5 some questions and suggestions for future works $\quad 115$

$\begin{array}{lr}\text { A Pictures } & 117\end{array}$

A.0.1 Geodesics in a special Randers space . . . . . . . . . . . . . . . . . . 117

$\begin{array}{ll}\text { Bibliography } & 119\end{array}$ 


\section{Introduction}

Finsler geometry is a classical branch of differential geometry started by P. Finsler in 1918 . Formally, a Finsler metric on a smooth manifold $M$ is a function $F: T M \rightarrow \mathbb{R}_{\geq 0}$ such that its restriction $F_{\mid T_{x} M}$ to every tangent space is a Minkowski norm. For example, a Riemannian metric $g_{i j}$ could be viewed as a Finsler metric with the function $F(x, y)=\sqrt{\sum_{i, j=1}^{n} g_{i j}(x) y^{i} y^{j}}$. There are many other important and well-studied classes of Finsler metric including the so-called Berward metric, Randers metric, $(\alpha, \beta)$-metric, Hilbert metric, and Funk metric [BCS12].

Recently, some efforts have been done to generalize some results from Riemannian spaces to the Finslerian ones. One of the interesting results in the study of transnormal functions and singular Riemannian foliations is due to Q. M. Wang [Wan87]. This topic is a classical subject in the Riemannian geometry, and lots of studies have been done in the Riemannian case [AT08, Ale04, Ale06, Ale10, ABT13, AR16b, AR16a, Miy13]. Indeed, the foliation in Riemannian manifolds is much better understood than that of the Finsler manifolds. In this work we try to generalize some of the results from the Riemannian case to the Finsler. In fact, we combine the classical methods in Finsler spaces obtained by the geometers with the Riemannian results to study this foliation. Roughly speaking, we start with the results in the Riemannian case and then using the tools in the Finsler space we generalize these results to the Finsler one. In recent years new tools in Finsler spaces appear that we apply some of them to achieve our goal. For example, Miguel [JS14], Robles [Rob07], and Shen [She01b] provided some powerful tools.

Definition. Let $f:(M, F) \longrightarrow \mathbb{R}$ be a smooth function. If there exists a continuous function $\mathfrak{b}: f(M) \longrightarrow \mathbb{R}$ such that

$$
F^{2}(\nabla f)=\mathfrak{b} \circ f
$$

then $f$ is called a Finsler transnormal (shortly F-transnormal) function.

Recall that a function $f: M \longrightarrow \mathbb{R}$ on a Riemannian manifold $(M, g)$ is called transnormal if there exists a continuous function $\mathfrak{b}: f(M) \longrightarrow \mathbb{R}$ such that $\|(\nabla f)\|^{2}=\mathfrak{b} \circ f$. In fact, the transnormal functions are the generalization of the distance functions. Observe that indeed the gradient of the function in the Finsler case cannot be calculated as much as easy the Riemannian case. Even in the Randers-Minkowski case it demands some efforts to be calculated. So one needs to use some other techniques to show that a function in $F$-transnormal. In this work some of the methods to calculate the norm of the gradient in some special cases are introduced.

On Riemannian transnormal functions, Wang [Wan87] proved that

Wang's Theorem. Let $M$ be a connected, complete, smooth Riemannian manifold and $f$ be a transnormal function on $M$. In other word $\|\nabla f\|^{2}=\mathfrak{b} \circ f$. We also assume that $\mathfrak{b}$ is of class $C^{2}$ on $f(M)$. Then 
the critical sets of $f$ are smooth submanifolds of $M$,

each regular level set of $f$ is a cylinder over either of the critical sets, and

the regular leaves are equifocal.

To have a better understanding of Wang's theorem see Picture 1 that exemplifies it. In this figure $f^{-1}(a)$ and $f^{-1}(b)$ are the critical sets.

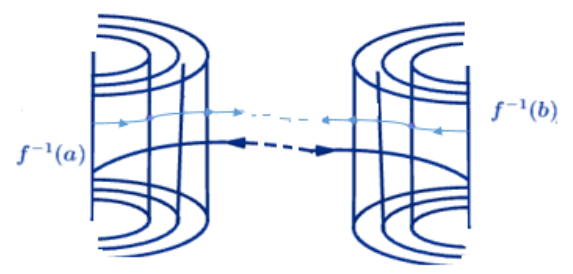

Figure 1: Wang's Theorem

In this work, we illustrated that this result is not valid in the Finsler case as it is in the Riemannian one. To generalize this result, we put some new natural hypothesis on it and then proved some version of this theorem for the Finsler space, in Chapter 3. In this chapter, we also proposed and proved some new theorems on foliation in Finsler spaces. Some examples to have a better understanding of the results are provided. In other word, in this work the following questions are answered:

Question 0.0.1. Given a Finsler manifold $(M, F)$, are the (regular) level sets of a F-transnormal function $f: M \longrightarrow \mathbb{R}$ parallel? If yes, is the distance between $f^{-1}(c)$ and $f^{-1}(d)$ described by $\int_{c}^{d} \frac{1}{\sqrt{\mathfrak{b}(s)}} d s$ as it was in the Riemannian case?

Question 0.0.2. Under which conditions the level sets of a transnormal function are equidistant?

Question 0.0.3. There are some conditions under which the level sets of a transnormal function on a Finsler manifold are level sets of singular Riemannian foliation for some Riemannian metric?

To answer these questions we prove the following results.

Proposition. Let $f: M \longrightarrow \mathbb{R}$ be a F-transnormal function with $f(M)=[a, b]$. If $c<d \in f(M)$ then

For every $q \in f^{-1}(d)$

$$
d_{F}\left(f^{-1}(c), q\right)=d_{F}\left(f^{-1}(c), f^{-1}(d)\right)=\int_{c}^{d} \frac{d s}{\sqrt{\mathfrak{b}(s)}}=L(\alpha)
$$

where $\alpha$ is a reparametrization of (the extension of) the integral curve of $\nabla f$.

(-) In particular $f^{-1}(c) \| f^{-1}(d)$.

Theorem. Let $f: M \longrightarrow \mathbb{R}$ be a F-transnormal and analytic function on a compact Finsler manifold $M$ with $f(M)=[a, b]$. Suppose that the level sets are connected and $a$ and $b$ are the only critical values and $\mathcal{F}=\left\{f^{-1}(c)\right\}_{c \in[a, b]}$ is a singular foliation. Then 


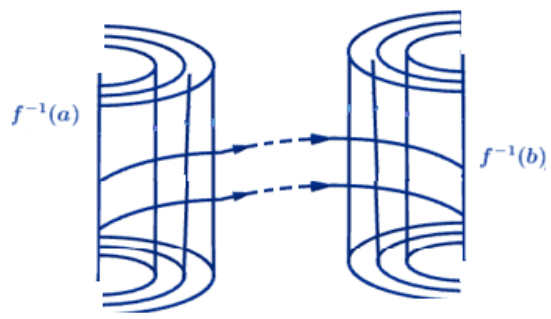

Figure 2: Proposition

there exist $r_{c, a}^{ \pm}, r_{c, b}^{ \pm}>0$ such that $f^{-1}(c)=C_{r_{c, a}^{ \pm}}^{ \pm}\left(f^{-1}(a)\right)=C_{r_{c, b}^{ \pm}}^{ \pm}\left(f^{-1}(b)\right)$, for every $c \in(a, b)$. In particular, for every $c, d \in(a, b), f^{-1}(c)$ is equidistant to $f^{-1}(d)$, i.e. $f^{-1}(c) \| f^{-1}(d)$ and $f^{-1}(d) \| f^{-1}(c)$. In other words, $\mathcal{F}=\left\{f^{-1}(c)\right\}_{c \in[a, b]}$ is a Finsler transnormal partition.

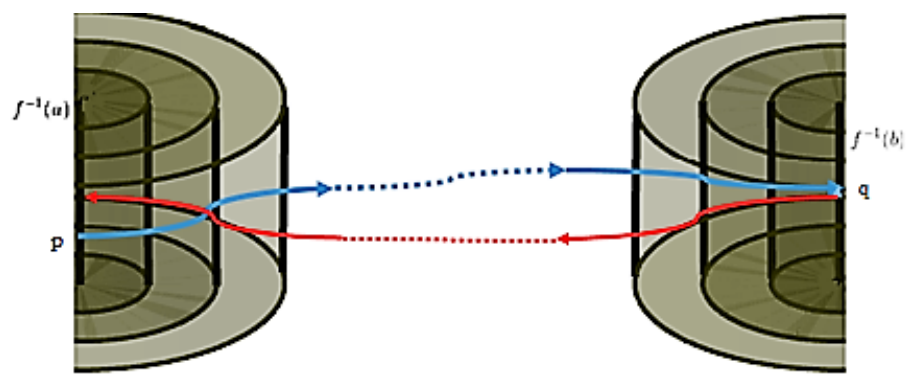

Figure 3: Theorem

Theorem. Let $f: M \longrightarrow \mathbb{R}$ be a F-transnormal and analytic function on a compact Finsler manifold $M$ with $f(M)=[a, b]$. Suppose that the level sets are connected and $a$ and $b$ are the only critical values and also the restriction of $f$ on the regular part is a Finsler foliation. Then

each regular level set $f^{-1}(c)$ is equifocal,

the critical level sets $f^{-1}(a)$ and $f^{-1}(b)$ are embedded submanifolds.

Proposition. Let $(M, F)$ be a compact Finsler manifold and $f: M \longrightarrow[a, b]$ be a F-transnormal function with $F^{2}(\nabla f)=\mathfrak{b}(f)$, where $\mathfrak{b}$ is a $C^{1}$ function on $[a, b]$, such that

(a) $\mathcal{F}=\left\{f^{-1}(c)\right\}_{c \in[a, b]}$ is a singular Finsler foliation,

(b) $a$ and $b$ are the only singular values at $[a, b]$,

(c) $\mathfrak{b}^{\prime}(a) \neq 0 \neq \mathfrak{b}^{\prime}(b)$

(d) and each of $f^{-1}(a)$ and $f^{-1}(b)$ has codimension greater than one.

Then there exists a Riemannian metric on $M$ such that $\mathcal{F}=\left\{f^{-1}(c)\right\}_{c \in[a, b]}$ is a singular Riemannian foliation.

In Chapter 1, we start with the necessary definitions and results that we need to accomplish the proofs and examples. Besides this the concepts of horizontal cone and geometrical cylinders are introduced and some properties of them are investigated. 
A particular case of Finsler metrics are Randers metrics with interesting applications in other sciences, like magnetic field [GW11], wildfire [Mar16], Zermelo's navigation problem [CG12], seismological studies [ABS03], etc. [Got09, CJS14, BRS ${ }^{+}$04, Voi11, PW12, Kri10, Jav12, BM07]. In Chapter 2, some properties of these spaces are studies and some results for the Randers transnormal functions are proved. Moreover, we equipped this chapter with some examples on the behavior of certain geodesics in the Randers spaces which together with the provided results will be used in the next chapters to clarify the results.

In Chapter 3, where the three listed questions above are answered, we state and prove the main results of this work.

In Chapter 4, some applications of certain results of Chapter 3 in Physics (Propagation of wildfire and water waves) are given.

In the last chapter, Chapter 5, some suggestions are provided to continue this work. 


\section{Chapter 1}

\section{Preliminaries}

\section{$1.1 \quad$ Introduction}

The goal of this chapter is to provide the necessary and elementary definitions and concepts that one needs to understand and accomplish the results. In the case it is of some interest, the proof of the results can be found in [BCS12, She01b, BR04, AIM13, She13]. Moreover the concepts of horizontal cone and geometric cylinders together with some properties of these concepts are provided in this chapter. Throughout this chapter we assume that $M$ is a smooth manifold.

\section{$1.2 \quad$ Metric spaces}

In this Section we cite three types of Finsler spaces including Randers and Minkowski spaces that we most deal with in this work.

\subsubsection{Minkowski spaces}

We start with Minkowski spaces which are the simplest regular flat spaces and are well known as a generalization of Euclidean spaces.

Definition 1.2.1. Given a vector space $V$, a non-negative function $F: V \longrightarrow[0, \infty)$ is called a Minkowski norm if it satisfies the following properties:

1. Fis smooth on $V \backslash\{0\}$

2. $F$ is positive homogeneous of degree 1 , that is $F(\lambda y)=\lambda F(y)$ for every $\lambda>0$

3. for each $y \in V \backslash\{0\}$, the fundamental tensor $g_{y}$ which is a symmetric bilinear form defined as follows is positive definite on $V$,

$$
g_{y}(u, v):=\left.\frac{1}{2} \frac{\partial^{2}}{\partial t \partial s}\left[F^{2}(y+t u+s v)\right]\right|_{s=t=0} .
$$

The pair $(V, F)$ is called a Minkowski space.

Observe that the forth condition above is equivalent saying that once we fix the direction $0 \neq y \in V, g_{y}$ is an inner product or in other words we have an inner product in $V$ which depends on the direction of $y$. In fact in Minkowski spaces one needs to take the direction into account. 
Remark 1.2.2. If for a Minkowski space we have that $F(y)=F(-y)$ it does not mean that this space is Euclidean. As an example consider $F: \mathbb{R}^{n} \longrightarrow \mathbb{R}$ with

$$
F(y)=\sqrt{\sum_{i=1}^{n}\left(y^{i}\right)^{2}+\sqrt{\sum_{i=1}^{n}\left(y^{i}\right)^{4}}}
$$

It is easy to check that it is a Minkowski type of the Finsler metric that is reversible which is not Euclidean.

Lemma 1.2.3. $F$ is convex on $V$, that is $F(u+v) \leq F(u)+F(v)$ for $u, v \in V$ and equality holds if and only if $u=\lambda v$ for some $\lambda \geq 0$

Definition 1.2.4. Let $\mathfrak{U} \subset \mathbb{R}^{n}$ be an open subset. A function $f: \mathfrak{U} \longrightarrow \mathbf{R}$ is positively homogeneous of degree $r$ at $p$ if and only if

$$
f(\lambda y)=\lambda^{r} f(y), \lambda>0 .
$$

Theorem 1.2.5 (Euler theorem). A $C^{1}$ function $f$ on $\mathfrak{U}$ is homogeneous of degree $r$ at $p$ if and only if

$$
\frac{\partial f}{\partial y_{i}} y^{i}=r f(y)
$$

Lemma 1.2.6. Assume that $f$ is a $C^{1}$ function in $\mathfrak{U}$. If $f$ is $r$-homogeneous at $p$, then $\frac{\partial f}{\partial y_{i}}$ is $(r-1)-$ homogeneous at $p$.

Corollary 1.2.7. If $f$ is homogeneous of degree 1 , we have $y^{i} f_{y^{i}}(y)=f(y)$ and by partial derivative of this relation one gets $\delta_{j}^{i} f_{y^{i}}+y^{i} f_{y^{i} y^{j}}=f_{y^{j}}$ and so

$$
y^{i} f_{y^{i} y^{j}}=0
$$

Remark 1.2.8. Observe that the condition of being positive homogeneous of degree 1 is necessary for the length of a curve to be independent of the positive reparametrization.

\section{Geometric properties}

We will proceed with some results that we accept most of them without any proof. For whom might be interested, the proof of these results are given in [Jav13] and [She01b].

Proposition 1.2.9. Given a Minkowski space $(V, F)$ and $y \in V$, the fundamental tensor $g_{y}$ is positive homogeneous of degree 0 , that is, $g_{\lambda y}=g_{y}$ for $\lambda>0$. Furthermore, $g_{y}(y, y)=F^{2}(y)$ and

$$
g_{y}(y, u)=\left.\frac{1}{2} \frac{\partial}{\partial s}\left[F^{2}(y+s u)\right]\right|_{s=0} .
$$

Proof. According to Euler theorem and Corollary 1.2.7, positive homogeneity of degree 0 of $g_{y}$ is clear. Now let's proof the other properties.

$$
\begin{aligned}
g_{y}(y, y) & =\left.\frac{1}{2} \frac{\partial^{2}}{\partial s \partial t}\left[F^{2}(y+s y+t y)\right]\right|_{s=t=0}=\left.\frac{1}{2} \frac{\partial^{2}}{\partial s \partial t}\left[(1+s+t)^{2} F^{2}(y)\right]\right|_{s=t=0} \\
& =F^{2}(y)
\end{aligned}
$$


which is one of the desired relations. Let's prove the last equation.

$$
\begin{aligned}
g_{y}(y, u) & =\left.\frac{1}{2} \frac{\partial^{2}}{\partial s \partial t}\left[F^{2}(y+s y+t u)\right]\right|_{s=t=0}=\left.\frac{1}{2} \frac{\partial^{2}}{\partial s \partial t}\left[(1+s)^{2} F^{2}\left(y+\frac{t}{1+s} u\right)\right]\right|_{s=t=0} \\
& =\left.\frac{1}{2} \frac{\partial}{\partial s}\left[(1+s)^{2} \frac{\partial}{\partial t} F^{2}(y+z u)\right]\right|_{s=t=0}=\left.\frac{1}{2} \frac{\partial}{\partial s}\left[(1+s) \frac{\partial}{\partial z} F^{2}(y+z u)\right]\right|_{s=z=0} \\
& =\left.\frac{1}{2} \frac{\partial}{\partial z} F^{2}(y+z u)\right|_{z=0},
\end{aligned}
$$

where $z=\frac{t}{1+s}$ and $\frac{\partial z}{\partial s}=-\frac{z}{1+s}$.

Lemma 1.2.10. (Cauchy-Schwarz inequality) Let $(V, F)$ be a Minkowski space. For any non zero $y \in V, g_{y}(y, v) \leq F(y) F(v)$ for every $v \in V$ and equality holds if and only if $v=\lambda y$ for some $\lambda \geq 0$.

Lemma 1.2.11. Let $(V, F)$ be a Minkowski space. If for non zero vectors $y, v \in V$ we have the equality $g_{y}(y, w)=g_{v}(v, w)$ for every $w \in V$, then $y=v$.

Proof. Taking $w=v$ in above equality and using Cauchy-Schwarz inequality one gets

$$
F^{2}(v)=g_{v}(v, v)=g_{y}(y, v) \leq F(y) F(v)
$$

As $F(v)>0$ it implies that $F(v) \leq F(y)$. Now by the same calculation for $w=y$ one gets

$$
F^{2}(y)=g_{y}(y, y)=g_{v}(v, y) \leq F(v) F(y)
$$

As $F(y)>0$ it implies that $F(y) \leq F(v)$. Hence $F(y)=F(v)$ which yields $g_{y}(y, v)=F(v) F(v)=$ $F(v) F(y)$ and finally by Cauchy-Schwarz $y=\lambda v$ for some $\lambda>0$ which concludes that $y=v$.

\subsubsection{Finsler Spaces}

Let us start with the definition of an indicatrix in a Finsler space. Observe that by the indicatrix at a point $p \in M$ we mean choosing the unit vectors in every direction at the origin of the space $T_{p} M$. The locus of the end points of all the unit lengths vector is called the indicatrix at $p$. In Euclidean geometry this locus is a (hyper-) sphere around 0. A formal definition is provided below.

Definition 1.2.12. Given a Finsler space $(M, F)$, the indicatrix of $F$ at a point $p \in M$ is the subset

$$
\Sigma^{F}=\left\{v \in T_{p} M \mid F(v)=1\right\}
$$

that is the unitary geometric sphere. Observe that the indicatrix does not look necessarily like a sphere. See Picture 1.1.

In Riemannian geometry the indicatrix is a quadratic surface around $0_{p}$, with coefficients equal to the fundamental tensor $h_{i j}$ which already exists in each point by the definition of the Riemannian metric [Ran41].

Roughly speaking by a Finsler metric we mean a norm on each tangent space $T_{p} M$ such that the indicatrix in $T_{p} M$ is a strictly convex submanifold which depends differentiably on the foot point. A more formal definition is given below. 

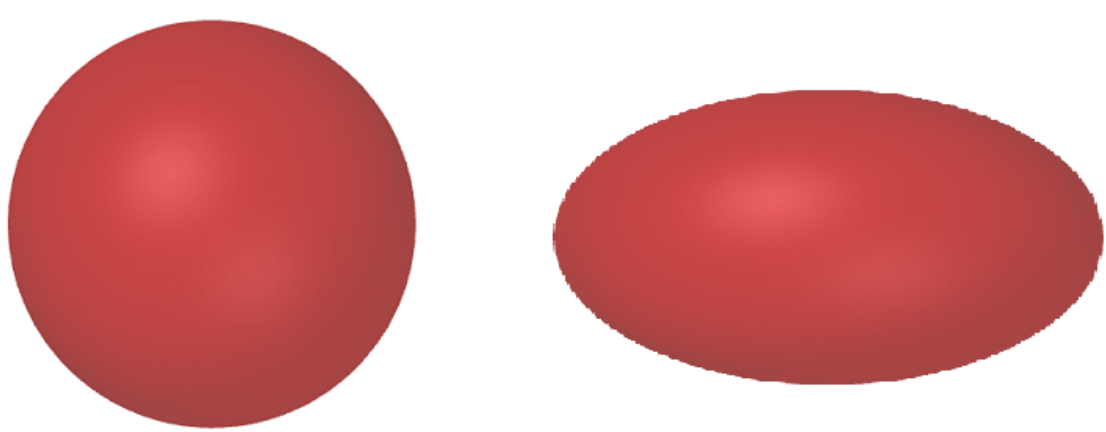

Figure 1.1

Definition 1.2.13. A function $F: T M \longrightarrow[0, \infty)$ is called a Finsler metric if it enjoys the following properties:

1. F is smooth on $T M \backslash\{0\}$

2. For each $x \in M, F_{x}:=\left.F\right|_{T_{x} M}$ is a Minkowski norm on $T_{x} M$.

The pair $(M, F)$ is called a Finsler space. $F$ is said to be a reversible (symmetric) Finsler metric if for every $y \in T M$ we have $F(-y)=F(y)$. Otherwise it is called non-reversible (non-symmetric). Note that not each reversible Finsler metric is a Riemannian one. As we saw that not each reversible Minkowski space is Euclidean.

It is worth mentioning that a Finsler metric is an object which lives on $T M$, not just on $M$. It means $F$ depends on both the foot point $x \in M$ and the direction $y \in T_{x} M$. Actually if we fix a point $x$ then $\left(T_{x} M, F_{x}\right)$ is a Minkowski space and if we fix a direction we have a Riemannian metric. Therefore if we fix both the foot point and the direction we have an Euclidean space.

Example 1.2.14. (Riemannian metric as a special type of Finsler metric) Given a Riemannian space $(M, g)$, one can get a Finsler metric by

$$
F_{x}(y)=\sqrt{g_{x}(y, y)}
$$

As each $g_{x}$ is an inner product in $T_{x} M, F_{x}$ is an Euclidean norm. The family of Euclidean norms $F=\left\{F_{x}\right\}_{x \in M}$ is a Finsler metric on $M$. Such a Finsler metric is called a Riemannian metric, namely $\left.F\right|_{T_{x} M}$ does not depend on the direction.

\section{Okubo technique}

Let $S$ be some smooth hypersurface in $\mathbb{R}^{n}$ that is defined by an equation $P(v)=0$. Suppose we want to find a function $F$ on $\mathbb{R}^{n}$ that has the constant value 1 on $S$. $F(v)$ is characterized by the equation

$$
P\left[\frac{v}{F(v)}\right]=0 .
$$

Occasionally, such an equation can be solved to give an explicit formula for $F(v)$. This method is known as Okubo's technique [BCS12]. It is useful when the indicatrix $S$ is specified by physical or geometric concerns, and one wants to recover the candidate Minkowski norm $F$ from the specified $S$. 
Lemma 1.2.15. Let $F_{1}$ and $F_{2}$ be two Finsler metrics on $M$ and $\Sigma^{F_{1}}(x)$ and $\Sigma^{F_{2}}(x)$, resp., the indicatrices at point $p \in M$ with respect to $F_{1}$ and $F_{2}$. If for every $x \in M$,

$$
\Sigma^{F_{1}}(x)=\Sigma^{F_{2}}(x)
$$

then the metrics are the same.

Proof. According to the fact

$$
\left\{y \in T_{x} M: F_{1}(y)=1\right\}=\Sigma^{F_{1}}(x)=\Sigma^{F_{2}}(x)=\left\{y \in T_{x} M: F_{2}(y)=1\right\},
$$

we have that the metrics coincide on the indicatrices. Hence for each $y \in T_{x} M$ we can write

$$
\begin{aligned}
F_{1}(y) & =F_{1}\left(F_{2}(y) \cdot \frac{y}{F_{2}(y)}\right)=F_{2}(y) F_{1}\left(\frac{y}{F_{2}(y)}\right) \\
& =F_{2}(y) F_{2}\left(\frac{y}{F_{2}(y)}\right)=F_{2}\left(F_{2}(y) \frac{y}{F_{2}(y)}\right)=F_{2}(y) .
\end{aligned}
$$

According to this lemma that is enough to find the Finsler metric on the indicatrix. In other words, if the restrictions of two Finsler metrics on the indicatrix coincide, the metrics are the same.

\subsubsection{Randers spaces}

In this section we just mention the definition of Randers spaces and as this matter is one of our main concern, we discuss about the details more in the next chapter.

Definition 1.2.16. Let $\alpha=\sqrt{a}$ and $\beta=a(., \mathcal{B})$ be a Riemannian metric and a 1-form on $M$, respectively. Here $\mathcal{B}$ is a vector field. If $a(\beta, \beta)<1$ then

$$
F=\alpha+\beta
$$

is a Randers metric.

Randers metrics are a special case of non-reversible Finsler metrics. Indeed from the relation $F(y)=\sqrt{a(y, y)}+a(y, \mathcal{B})$, it can be easily checked that $F(y) \neq F(-y)$. To have a better understanding of the definition note that a Randers metric is obtained by adding a linear form to a Riemannian metric and the relation $a(\beta, \beta)<1$ assures that the resulting Minkowski norm $F_{x}:=\left.F\right|_{T_{x} M}$ is positive and the fundamental tensor $g_{y}$ is positive definite. Picture 1.2 shows the relations between the above-mentioned metrics.

\subsubsection{Length Structure}

Given a Finsler metric $F: T M \longrightarrow \mathbb{R}^{+}$, we define the length of a piecewise smooth curve $\gamma:[a, b] \longrightarrow M$ with respect to $F$ as

$$
L_{F}(\gamma)=\int_{a}^{b} F\left(\gamma(t), \gamma^{\prime}(t)\right) d t
$$




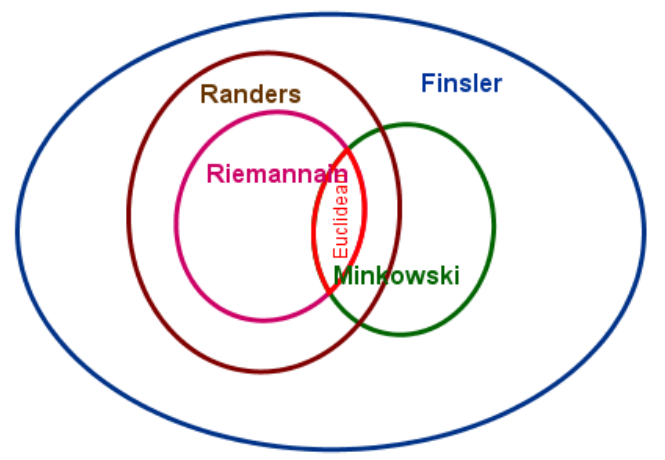

Figure 1.2: The metrics

Lemma 1.2.17. Suppose that we have a change of parametrization $t=\phi(s)$ for which $\frac{d \phi}{d s}>0 . L_{F}$ is independent of the choice of parameter if and only if

$$
F(x, \lambda y)=\lambda F(x, y), \lambda>0 .
$$

Proof. Let $\gamma(t)=(x(t))$. As the length is invariant under the reparametrization,

$$
\int_{a}^{b} F\left(x, x^{\prime}(t)\right) d t=\int_{a}^{b} F\left(x, x^{\prime}(s) \frac{d s}{d t}\right) d t=\int_{a}^{b} F\left(x, x^{\prime}(s)\right) \frac{d s}{d t} d t
$$

Now, by the continuity of $F$, we get

$$
F\left(x, x^{\prime}(s) \frac{d s}{d t}\right)=F\left(x, x^{\prime}(s)\right) \frac{d s}{d t}
$$

which means $F(x, \lambda y)=\lambda F(x, y), \lambda>0$. The reverse direction has a straightforward proof.

Consider the distance function $d_{x}(z):=d_{F}(x, z), z \in M$. Thus $d_{x}^{2}$ is smooth nearby $x$ and $C^{1}$ at $x$. Also for $z$ nearby $x$,

$$
d_{z}(x)=F\left(x, \exp _{x}^{-1}(z)\right),
$$

which implies that $d_{x}$ is smooth nearby $x$. Observe that in a Riemannian space $(M, h)$, the distance function $d_{x}(z):=d_{h}(x, z)$ is smooth every where on a local neighborhood except in $x$. However $d_{x}^{2}$ is smooth every where [She01b].

Proposition 1.2.18. [She01b] Let $(M, F)$ be a Finsler space. Suppose that $d_{x}^{2}$ is $C^{2}$ at $x$, then $F_{x}$ is Euclidean at $x$. Thus $d_{x}^{2}$ is $C^{2}$ for all $x \in M$ if and only if $F$ is Riemannian.

Similar to the Riemannian space, the distance from a point $p \in M$ to another point $q \in M$ in the Finsler space $(M, F)$ is defined by

$$
d_{F}(p, q):=\inf _{\gamma} \int_{0}^{1} F\left(\gamma(t), \gamma^{\prime}(t)\right) d t
$$

where the infimum is taken over all piecewise smooth curves $\gamma:[0,1] \longrightarrow M$ joining $p$ to $q$. Observe that this is a non-symmetric distance, namely, the distance from $p$ to $q$ may not be equal to the distance from $q$ to $p$. If for each pair $(p, q)$ these distances are the same, then the metric must be Riemannian. 


\subsection{Geometric Objects}

In this part we talk about the geometric objects that we need in the next chapters to prove the results. Some concepts are new and so some of their necessary properties are given.

\subsection{1 horizontal cone}

Here we introduce the concept of the horizontal cone $\mathfrak{C}_{p}$ that is a generalization of the orthogonal space to a submanifold $L \subset M$ at $p \in L$ from Riemannian spaces to the Finslerian ones.

Definition 1.3.1. Let $L$ be a submanifold of $M$ and $p \in L$. The horizontal cone to $L$ at $p$ is defined as

$$
\mathfrak{C}_{p}(L)=\left\{0 \neq v \in T_{p} M: g_{v}(v, u)=0, \text { for every } u \in T_{p} L\right\} .
$$

Namely $v \in \mathfrak{C}_{p}(L)$ if it is orthogonal to the submanifold $L$ at the direction of itself.

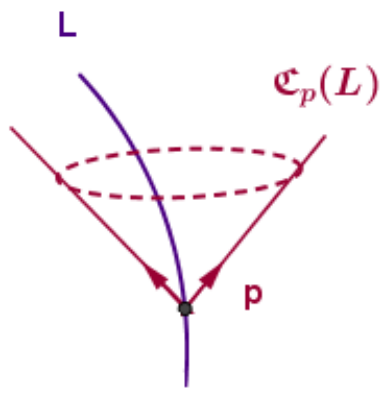

Figure 1.3: the horizontal cone $\mathfrak{C}_{p}(L)$

As by definition of Finsler spaces $g_{v}$ is an inner product and by Proposition 1.2.9 it is positive homogeneous of degree zero we have that for every $v \in \mathfrak{C}_{p}, u \in T_{p} L$, and $\lambda>0$,

$$
g_{\lambda v}(\lambda v, u)=\lambda g_{v}(v, u)=\lambda .0=0,
$$

which means $\lambda v \in \mathfrak{C}_{p}$. This is why we use the expression of cone to indicate the orthogonal set to a submanifold and notice that for $v \in \mathfrak{C}_{p},-v$ may not be in $\mathfrak{C}_{p}$ (it can be seen in example 2.4.1). Hence, in the general case $\mathfrak{C}_{p}$ is not a vector space however we will see under some conditions it is.

Lemma 1.3.2. Assume that $(M, F)$ is a Finsler manifold of dimension n. Given a k-dimensional submanifold $L$ of $(M, F)$, for every $p \in L$,

1. $\mathfrak{C}_{p}(L)$ is a submanifold of $T_{p} M$ of dimension $n-k$ and in particular $\mathfrak{C}(L)=\cup_{p \in L} \mathfrak{C}_{p}(L)$ is a submanifold of $T M$ of dimension $n$,

2. let $\mathfrak{C}_{p}^{1}(L)=\mathfrak{C}_{p}(L) \cap \Sigma^{F}(p)$, then $\mathfrak{C}_{p}^{1}(L)$ is a submanifold of $\mathfrak{C}_{p}(L)$ of dimension $n-k-1$ and in particular $\mathfrak{C}^{1}(L)=\cup_{p \in L} \mathfrak{C}_{p}^{1}(L)$ is a submanifold of dimension $n-1$.

Proof. Fix $p \in L$. Let us first prove that $\mathfrak{C}_{p}(L)$ is a $(n-k)$-dimensional submanifold of $T_{p} M$. Consider a basis $\left\{u_{1}, \ldots, u_{k}\right\}$ of $T_{p} L$ and define the application

$$
\begin{aligned}
\lambda: T_{p} M & \backslash 0 \rightarrow \mathbb{R}^{k} \\
v & \mapsto\left(g_{v}\left(v, u_{1}\right), \ldots, g_{v}\left(v, u_{k}\right)\right) .
\end{aligned}
$$


Observe that $\lambda$ is smooth and $\lambda^{-1}(0)=\mathfrak{C}_{p}(L)$. Let us show that 0 is a regular value of $\lambda$. That is enough to show that $d \lambda_{v}: T_{v}\left(T_{p} M \backslash 0\right) \longrightarrow \mathbb{R}^{k}$ is surjective for every $v \in \mathfrak{C}_{p}(L)$. Given $v \in \mathfrak{C}_{p}(L)$, for every $l \in\{1, \ldots, k\}$, we have

$$
\begin{aligned}
d \lambda_{v}\left(u_{l}\right) & =\left.\frac{d}{d t} \lambda\left(v+t u_{l}\right)\right|_{t=0}=\left(\left.\frac{d}{d t} g_{v+t u_{l}}\left(v+t u_{l}, u_{1}\right)\right|_{t=0}, \ldots,\left.\frac{d}{d t} g_{v+t u_{l}}\left(v+t u_{l}, u_{k}\right)\right|_{t=0}\right) \\
& =\left.\frac{d}{d t}\left(\frac{1}{2} \frac{d}{d s} F^{2}\left(v+t u_{l}+s u_{1}\right), \ldots, \frac{1}{2} \frac{d}{d s} F^{2}\left(v+t u_{l}+s u_{k}\right)\right)\right|_{s=t=0} \\
& =\left(g_{v}\left(u_{l}, u_{1}\right), \ldots, g_{v}\left(u_{l}, u_{k}\right)\right) .
\end{aligned}
$$

Consider the set $\left\{d \lambda_{v}\left(u_{1}\right), \ldots, d \lambda_{v}\left(u_{k}\right)\right\}$. Now as the vectors $\left\{u_{1}, \ldots, u_{k}\right\}$ are linearly independent, the Gramian determinant

$$
G\left(u_{1}, \ldots, u_{k}\right)=\left|\begin{array}{cccc}
\left\langle u_{1}, u_{1}\right\rangle & \left\langle u_{1}, u_{2}\right\rangle & \ldots & \left\langle u_{1}, u_{k}\right\rangle \\
\left\langle u_{2}, u_{1}\right\rangle & \left\langle u_{2}, u_{2}\right\rangle & \ldots & \left\langle u_{2}, u_{k}\right\rangle \\
\vdots & \vdots & \ddots & \vdots \\
\left\langle u_{k}, u_{1}\right\rangle & \left\langle u_{k}, u_{2}\right\rangle & \ldots & \left\langle u_{k}, u_{k}\right\rangle
\end{array}\right|
$$

where $\langle.,\rangle=.g_{v}(.,$.$) , is nonzero. It means \left\{d \lambda_{v}\left(u_{1}\right), \ldots, d \lambda_{v}\left(u_{k}\right)\right\}$ is a linearly independent set in $\mathbb{R}^{k}$ and so a basis of it. In particular it implies that $d \lambda_{v}$ is surjective. So according to Implicit Function Theorem $\mathfrak{C}_{p}(L)$ is an embedded submanifold of dimension $n-k$. Now as $\mathfrak{C}(L)$ can be locally seen as a product of $L \times \mathfrak{C}_{p}(L)$, it is a submanifold of dimension $(n-k)+k=n$.

Let us show that $\mathfrak{C}_{p}^{1}(L)$ is a submanifold. Define

$$
\begin{aligned}
\beta: \mathfrak{C}_{p}(L) \longrightarrow \mathbb{R} \\
v \mapsto F(v) .
\end{aligned}
$$

Then $\beta^{-1}(1)=\mathfrak{C}_{p}^{1}(L)$. According to Implicit Function Theorem, that is sufficient to show that 1 is a regular value of $\beta$. That is $d \beta_{v}: T_{v}\left(\mathfrak{C}_{p}(L)\right) \longrightarrow \mathbb{R}$, for every $v \in \mathfrak{C}_{p}^{1}(L)$, is surjective. Note that

$$
d \beta_{v}(v)=\left.\frac{d}{d t} \beta(v+t v)\right|_{t=0}=\left.\frac{d}{d t} F(v+t v)\right|_{t=0}=\left.\frac{d}{d t}(1+t) F(v)\right|_{t=0}=F(v)=1 .
$$

Here we used the positive homogeneity of $F$. So $d \beta_{v}$ is surjective and consequently $\mathfrak{C}_{p}^{1}(L)$ is an embedded submanifold of $\mathfrak{C}_{p}(L)$ of dimension $n-k-1$. As $\mathfrak{C}^{1}(L)$ can be locally seen as a product of $L \times \mathfrak{C}_{p}^{1}(L)$, it is a submanifold of dimension $(n-k-1)+k=n-1$.

\subsubsection{Chern connection}

In this section we introduce the concepts of Chern connection, Cartan tensor and covariant derivative, based on [JS14] and then mention some useful properties that we use later. In Finsler geometry, in order to define the connection, one needs to differentiate the metric three times from which the Cartan tensor is obtained that is defined as the following trilinear form: 


$$
C_{v}\left(w_{1}, w_{2}, w_{3}\right)=\left.\frac{1}{2} \frac{\partial}{\partial s} g_{v+s w_{1}}\left(w_{2}, w_{3}\right)\right|_{s=0}=\left.\frac{1}{4} \frac{\partial^{3}}{\partial s_{3} \partial s_{2} \partial s_{1}} F^{2}\left(v+\sum_{i=1}^{3} s_{i} w_{i}\right)\right|_{s_{1}=s_{2}=s_{3}=0}
$$

for every $v, w_{1}, w_{2}, w_{3} \in T_{\pi(v)} M$ with $v \neq 0$.

Lemma 1.3.3. For every $v \in T_{p} M \backslash 0$, the Cartan tensor has the following properties:

1. $C_{v}$ is symmetric;

2. $C_{v}$ is positive homogeneous of degree -1 , that is, $C_{\lambda v}=\frac{1}{\lambda} C_{v}$, for every $\lambda>0$;

3. $C_{v}(v, u, w)=C_{v}(u, v, w)=C_{v}(u, w, v)=0, u, w \in T_{p} M$.

Definition 1.3.4. Let $(M, F)$ be a Finsler space and $V$ a smooth vector field defined on an open $\mathcal{U} \subset M$ such that $V$ does not have any singularities in $\mathcal{U}$. Consider a linear connection $\nabla^{V}$ in the tangent bundle TU. We say that

1. $\nabla^{V}$ is torsion-free if $[X, Y]=\nabla_{X}^{V} Y-\nabla_{Y}^{V} X$ for every $X, Y \in \mathfrak{X}(\mathcal{U})$,

2. $\nabla^{V}$ is almost $g$-compatible if $X g_{V}(Y, Z)=g_{V}\left(\nabla_{X}^{V} Y, Z\right)+g_{V}\left(Y, \nabla_{X}^{V} Z\right)+2 C_{V}\left(\nabla_{X}^{V} V, Y, Z\right)$ for every $X, Y, Z \in \mathfrak{X}(\mathcal{U})$,

where $g_{V}$ and $C_{V}$ are, respectively, the fundamental tensor and the Cartan tensor of $F$ evaluated in the vector field $V$.

Proposition 1.3.5. Given a smooth vector field $V$ defined on an open $\mathcal{U} \subset M$ which has no singularities on $\mathcal{U}$, there exists a unique affine connection $\nabla^{V}$ that is almost g-compatible and torsion-free. This connection is called the Chern connection and is determined by a Koszul formula as

$$
\begin{aligned}
2 g_{V}\left(\nabla_{X}^{V} Y, Z\right) & =X g_{V}(Y, Z)-Z g_{V}(X, Y)+Y g_{V}(Z, X) \\
& +g_{V}([X, Y], Z)+g_{V}([Z, X], Y)-g_{V}([Y, Z], X) \\
& +2\left(-C_{V}\left(\nabla_{X}^{V} V, Y, Z\right)-C_{V}\left(\nabla_{Y}^{V} V, Z, X\right)+C_{V}\left(\nabla_{Z}^{V} V, X, Y\right)\right) .
\end{aligned}
$$

Remark 1.3.6. The Chern connection have the following properties

1. $\nabla^{\lambda V}=\nabla^{V}$ for every $\lambda>0$, that is $\nabla^{V}$ is positive homogeneous of degree zero in $V$,

2. for every $p \in \mathcal{U}, \nabla^{V(p)}$ just depends on $v=V(p)$.

Corollary 1.3.7. Given a Finsler space $(M, F)$ and whose Chern connection $\nabla^{V}$, let $\hat{\nabla}$ be the Levi-Civita connection of the Riemannian metric $\hat{g}:=g_{V}$. Then

$$
\nabla_{V}^{V} V=\hat{\nabla}_{V} V
$$

In particular, a regular curve $\gamma:[a, b] \rightarrow M$ is a geodesic of $F$ (see Definition 1.3.10) if and only if it is a geodesic of Riemannian metric $\hat{g}$, for every local extension and without singularity $V$ of $\gamma^{\prime}$. 
Proof. According to Koszul formulas, in the Finsler and Riemannian cases we have

$$
\begin{aligned}
2 g_{V}\left(\nabla_{V}^{V} V, Z\right) & =V g_{V}(V, Z)-Z g_{V}(V, V)+V g_{V}(Z, V) \\
& +g_{V}([V, V], Z)+g_{V}([Z, V], V)-g_{V}([V, Z], V) \\
& =2 g_{V}\left(\hat{\nabla}_{V} V, Z\right),
\end{aligned}
$$

for every $Z \in \mathfrak{X}(\mathcal{U})$. In other words we have $\hat{g}\left(\nabla_{V}^{V} V, Z\right)=\hat{g}\left(\hat{\nabla}_{V} V, Z\right)$ for every $Z \in \mathfrak{X}(\mathcal{U})$ completing the proof.

Definition 1.3.8. Given a smooth curve $\gamma:[a, b] \rightarrow \mathcal{U}$, for a vector field $V \in \mathfrak{X}(M)$ along $\gamma$ there exists a unique application $D_{\gamma}^{V}: \mathfrak{X}(\gamma) \rightarrow \mathfrak{X}(\gamma)$ given by $X \mapsto D_{\gamma}^{V} X$ such that

1. $D_{\gamma}^{V}(X+Y)=D_{\gamma}^{V}(X)+D_{\gamma}^{V}(Y)$,

2. $D_{\gamma}^{V}(f X)=f^{\prime}(t) X(t)+f(t) D_{\gamma}^{V} X$

3. $D_{\gamma}^{V} X=\nabla_{\gamma^{\prime}}^{V} X$,

for every $X, Y \in \mathfrak{X}(\gamma), t \in[a, b]$, and $f \in \mathcal{F}([a, b])$.

Definition 1.3.9. A vector field $X$ along a regular curve $\gamma:[a, b] \rightarrow(M, F)$ is said parallel if

$$
D_{\gamma}^{\gamma^{\prime}} X=0
$$

Given $v \in T_{\gamma(a)} M \backslash\{0\}$ there exists a unique vector field $X$ with $X(a)=v$ which is parallel along $\gamma$. Furthermore the parallel transport of $v$ along $\gamma$, that is the application which associates to each $(t, v) \in[a, b] \times T_{\gamma(a)} M$ the vector $X_{v}(t) \in T_{\gamma(t)} M$, is well defined.

\subsubsection{Geodesic and exponential map}

Given a Finsler space $(M, F)$, the geodesics of $F$ are the time minimizing curves. So one should find the extremal of the functional 1.2 and this leads us to a geodesic of $F$.

Definition 1.3.10. A smooth curve $\gamma:[a, b] \rightarrow M$ is a geodesic of $(M, F)$ if

$$
D_{\gamma}^{\gamma^{\prime}} \gamma^{\prime}=0
$$

on $[a, b]$.

Definition 1.3.11. A Finsler manifold $(M, F)$ is said to be forward (resp. backward) geodesically complete if every geodesic $\gamma:[0,1) \longrightarrow M$, parametrized to have constant Finsler speed, can be extended to a geodesic defined on $\gamma:[0,+\infty) \longrightarrow M$ (resp. $(-\infty, 0])$.

Lemma 1.3.12. Let $\gamma:[a, b] \rightarrow M$ be a geodesic and $X$ and $Y$ be vector fields along $\gamma$. Then

$$
\frac{d}{d t} g_{\gamma^{\prime}}(X, Y)=g_{\gamma^{\prime}}\left(D_{\gamma}^{\gamma^{\prime}} X, Y\right)+g_{\gamma^{\prime}}\left(X, D_{\gamma}^{\gamma^{\prime}} Y\right)
$$

which means the Chern connection is g-compatible in the direction of the geodesic. 
Note that if $\gamma$ is a geodesic, the curve $\tilde{\gamma}(t)=\gamma(-t)$ may not be a geodesic anymore. It means the geodesic joining a point $A$ to a point $B$ may not coincide with the geodesic joining $B$ to $A$.

Let us define the energy functional associated to a Finsler space $(M, F)$ for any piecewise smooth curve $\gamma:[a, b] \longrightarrow M$ as

$$
E(\gamma)=\frac{1}{2} \int_{a}^{b} F^{2}\left(\gamma^{\prime}\right) d t
$$

Denote by $C(P, Q)$ the subset of piecewise smooth curves $\gamma:[a, b] \longrightarrow M$ joining the submanifold $P$ to the submanifold $Q$. Namely

$$
C(P, Q)=\{\gamma:[a, b] \longrightarrow M: \gamma(a) \in P \text { and } \gamma(b) \in Q\}
$$

Lemma 1.3.13. [JS14] Let $\gamma \in C(P, Q)$. Then $\gamma$ is a critical point of the energy functional $\left.E\right|_{C(P, Q)}$ if and only if $\gamma$ is a geodesic $g_{\gamma^{\prime}}$-orthogonal to $P$ and $Q$.

Lemma 1.3.14. [BCS12] Assume that $(M, F)$ is a Finsler space. Then for every $v \in T_{p} M \backslash\{0\}$ with $F(v)<\epsilon$, there exists a unique geodesic $\gamma_{v}:(-2,2) \rightarrow M$ such that $\gamma_{v}^{\prime}(0)=v$.

Suppose we are given a geodesic $\gamma_{v}:(-2,2) \rightarrow M$ and any positive constant $\lambda$. The chain rule allows us to claim that the curve $\gamma_{v}:\left(\frac{-2}{\lambda}, \frac{2}{\lambda}\right) \rightarrow M$ is also a geodesic which passes through $\pi(v)$ at time 0 , but the velocity at that moment is $\lambda v$. By uniqueness, the said curve must be $\gamma_{\lambda v}(t)$. So one can write

$$
\gamma_{\lambda v}(t)=\gamma_{v}(\lambda t)
$$

Definition 1.3.15. Define the exponential as

$$
\exp _{x}(v)=\left\{\begin{array}{cc}
\gamma_{v}(1) & , \quad v \neq 0 \\
x & , \quad v=0
\end{array}\right.
$$

where $\gamma_{v}(t)$ is the unique geodesic introduced in Lemma 1.3.14. In particular like the Riemannian case one can write

$$
\exp _{x}(t v)=\gamma_{v}(t)
$$

Definition 1.3.16. The geodesic sphere of radius $r$ at point $p \in M$ is defined by

$$
\mathcal{S}_{r}(p)=\exp _{p}\left(\Sigma_{r}^{F}(p)\right)
$$

where $\Sigma_{r}^{F}(p)=\left\{v \in T_{p} M \mid d_{F}\left(0_{p}, v\right)=r\right\}$.

Proposition 1.3.17. [She01b] Let $(M, F)$ be a Finsler space. The exponential map exp is $C^{1}$ on the zero sections of $\pi: T M \longrightarrow M$ and for $x \in M, d\left(\exp _{x}\right)_{0}: T_{x} M \longrightarrow T_{x} M$ is the identity map.

According to this Proposition $\exp _{x}$ is $C^{\infty}$ on $T_{x} M \backslash\{x\}$ and $C^{1}$ at $0 \in T_{x} M$. Indeed $\exp _{x}$ is a diffeomorphism on a neighborhood of $x$ in $T_{x} M \backslash\{x\}$.

\subsubsection{Jacobi field and conjugate and focal points}

In this part, which is written based on [JS14], we give some necessary definitions and facts about the Jacobi fields in Finsler spaces. Let us prepare the minds with some concepts associated to submanifolds. 
Given a Finsler space $(M, F)$ and a submanifold $L \subset M$, denote the tangent bundle of $L$ as $T L$ and consider the horizontal cone $\mathfrak{C}(L)$ of $L$ which contains the vectors $v \in T M \backslash\{0\}$ such that $\pi(v) \in L$ and $v \in \mathfrak{C}_{\pi(v)}(L)$. Since we already have mentioned it, the horizontal cone at $\pi(v)$, i.e. $\mathfrak{C}_{\pi(v)}(L)$ may not be a vector space and so the horizontal cone $\mathfrak{C}(L)$ may not be a fiber bundle. Indeed what we have is a submersion

$$
\pi: \mathfrak{C}(L) \rightarrow L
$$

in which $\pi$ is the natural projection of $T M$ restricted on $\mathfrak{C}(L)$. Let us denote the smooth sections of $\pi: T L \rightarrow L$ with $\mathfrak{X}(L)$ and the smooth sections of $\pi: \mathfrak{C}(L) \rightarrow L$ with $\mathfrak{X}(L)^{\perp}$. Moreover, given $\xi \in \mathfrak{X}(L)^{\perp}$ we denote by $\mathfrak{X}(L)_{\xi}^{\perp}$ the smooth sections of $\pi: T M \rightarrow L$, such that for every $p \in L$, $W(p)$ is $g_{\xi(p)}$-orthogonal to $T_{p} L$, i.e. $g_{\xi}(W(p), u)=0$, for every $u \in T_{p} L$. Let us denote this space with $\mathfrak{C}_{p}^{\xi}(L)$. That is

$$
\mathfrak{C}_{p}^{\xi}(L)=\left\{w \in T_{p} M \mid g_{\xi}(w, u)=0, \forall u \in T_{p} L\right\} .
$$

In order to prevent any confusion let us give some explanation about $\mathfrak{C}_{p}^{\xi}(L)$ and $\mathfrak{C}_{p}(L)$. We say that $\xi(p) \in \mathfrak{C}_{p}(L)$ if it is orthogonal to $L$ with respect to the inner product $g_{\xi(p)}$. Indeed, the inner product depends on $\xi(p)$ and it changes when $\xi(p)$ changes. However, $W(p) \in \mathfrak{C}_{p}^{\xi}(L)$ if it is orthogonal to $L$ with respect to the special inner product $g_{\xi(p)}$. Indeed we first fix the inner product and then we start working with it. Observe that in particular $\mathfrak{C}_{p}^{\xi}(L)$ is closed under addition and scalar multiplication and so it is a vector space. In addition $\xi \in \mathfrak{X}(L) \frac{\perp}{\xi}$. See Picture 1.4. In the case

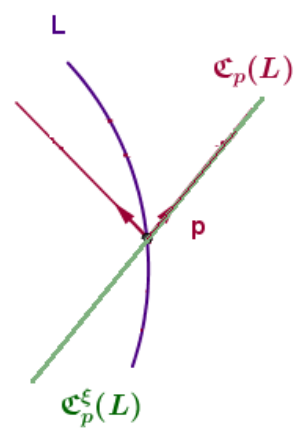

Figure 1.4

that $\left.g_{\xi}\right|_{T_{p} L \times T_{p} L}$ is non-degenerate we have the following decomposition.

$$
T_{p} M=T_{p} L \oplus \mathfrak{C}_{p}^{\xi}(L)
$$

Hence for every smooth sections $V$ of $\pi: T M \rightarrow L$, we can define $\tan _{\xi}(V)$ and $\operatorname{nor}_{\xi}(V)$ as the vector fields in $\mathfrak{X}(L)$ obtained in every $p \in L$ projecting $V_{p}$ to $T_{p} L$ and $\mathfrak{C}_{p}^{\xi}(L)$, resp., through the decomposition 1.6.

Definition 1.3.18. Let $V$ be a non-zero vector field defined in an open subset $\mathcal{U} \subset M$. Being $\nabla^{V}$ the Chern connection having $V$ as a reference vector field, the curvature $R^{V}$ associated to the affine connection $\nabla^{V}$ which is a $(1,3)$ tensor in $\mathcal{U}$ is defined as

$$
R^{V}(X, Y) Z=\nabla_{X}^{V} \nabla_{Y}^{V} Z-\nabla_{Y}^{V} \nabla_{X}^{V} Z-\nabla_{[X, Y]} Z
$$

for every $X, Y, Z \in \mathfrak{X}(\mathcal{U})$.

Definition 1.3.19. Fix $\xi \in \mathfrak{X}(L)^{\perp}$ and suppose that $\left.g_{\xi}\right|_{T_{p} L \times T_{p} L}$ is non-degenerate for every $p \in L$. 
The normal second fundamental form $S_{\xi}: \mathfrak{X}(L) \longrightarrow \mathfrak{X}(L)$ is defined as

$$
S_{\xi}(\mathcal{U})=\tan _{\xi} \nabla_{\mathcal{U}}^{\xi} \xi
$$

Definition 1.3.20. Given a geodesic $\gamma:[a, b] \longrightarrow M$, a Jacobi field along $\gamma$ is a vector field $J$ along $\gamma$ satisfying

$$
J^{\prime \prime}=R^{\gamma}\left(\gamma^{\prime}, J\right) \gamma^{\prime}
$$

Moreover, for a submanifold $L \subset M$ such that $\gamma(a) \in L$ and $\gamma^{\prime}$ is $g_{\gamma^{\prime}(a)}$ - orthogonal to $L$, a Jacobi field $J$ is said to be $L$-Jacobi field if $\tan _{\gamma^{\prime}} J^{\prime}(a)=S_{\gamma^{\prime}}(J(a))$. Similar to the Riemannian case an instant $t_{0} \in(a, b]$ is

(i) conjugate if there exists a Jacobi field $J$ along $\gamma$ such that $J(a)=J\left(t_{0}\right)=0$.

(ii) $L$-focal if there exists a $L-J a c o b i$ field $J$ such that $J\left(t_{0}\right)=0$.

Lemma 1.3.21. [She01b] Let $(M, F)$ be a Finsler space and $\gamma:[0, l] \rightarrow M$ a geodesic of it. Given a variation $f:(-\epsilon, \epsilon) \times[0, l] \rightarrow M$ of $\gamma$, namely for each $s \in(-\epsilon, \epsilon), t \rightarrow f(s, t)$ is a geodesic and in particular $f(0, t)=\gamma(t)$, let

$$
J(t)=\frac{\partial f}{\partial s}(0, t)
$$

Then $J$ is a Jacobi field along $\gamma$ which means it satisfies EQ. 1.8. Conversely for every vector field $J$ along $\gamma$ satisfying the Jacobi $E Q .1 .8$, there is a geodesic variation $f(s, t)$ of $\gamma$ whose variation field is equal to $J(t)$.

Lemma 1.3.22. Let $\gamma:[a, b] \rightarrow M$ be a geodesic of $(M, F)$ with $\gamma(a)=p$. Then for any $v, w \in T_{p} M$, there exists a unique Jacobi field $J$ such that $J(a)=v$ and $J^{\prime}(a)=w$.

Lemma 1.3.23. Let $\gamma:[a, b] \rightarrow M$ be a geodesic of $(M, F)$ with $\gamma(a)=p$. Assume that $v \in T_{p} M$ belongs to the domain of $\exp _{p}$. Then for any $w \in T_{v}\left(T_{p} M\right)$, we have

$$
d\left(\exp _{p}\right)_{v} w=J(1)
$$

where $J$ is the Jacobi field along $\gamma$ with $J(0)=0$ and $J^{\prime}(0)=w$.

Lemma 1.3.24. consider a vector field $J$ along a geodesic. If $J$ is a Jacobi field then

(i) $J$ is tangent to $\gamma$ if and only if $J(t)=(a t+b) \gamma^{\prime}$

(ii) the following statements are equivalent.

(a) $g_{\gamma^{\prime}}\left(\gamma^{\prime}, J\right)=0$

(b) there exists a,b such that $g_{\gamma^{\prime}}\left(\gamma^{\prime}(a), J(a)\right)=g_{\gamma^{\prime}}\left(\gamma^{\prime}(b), J(b)\right)=0$

(c) there exists a such that $g_{\gamma^{\prime}}\left(\gamma^{\prime}(a), J(a)\right)=g_{\gamma^{\prime}}\left(\gamma^{\prime}(a), J^{\prime}(a)\right)=0$

(iii) if $\gamma$ is non-null, i.e. $F\left(\gamma^{\prime}\right) \neq 0$, then $J$ is a Jacobi field if and only if nor $\gamma_{\gamma^{\prime}} J$ and $\tan _{\gamma^{\prime}} J$ are Jacobi fields.

Let us continue this part with a Lemma which is the goal of this part and it will be used to prove on of the main results of the work. 
Lemma 1.3.25. Given a Finsler space $(M, F)$ and a submanifold $L$ of $M$, let $\exp ^{\perp}$ denote the restriction of $\exp$ to $\mathfrak{C}(L)$. Then $q \in M$ is a L-focal point if and only if it is a critical value of $\exp ^{\perp}$.

Proof. Notice that as $q$ is a $L$-focal point, according to Definition 1.3.20, there exists a geodesic $\gamma:[0, l] \rightarrow M$ that is $g_{\gamma^{\prime}}$-orthogonal to $L$ with $\gamma(0) \in L$ and $\gamma(l)=q$ and a variation $f:$ $(-\epsilon, \epsilon) \times[0, l] \rightarrow M$ of $\gamma$ with the following properties

(1) for each $s \in(-\epsilon, \epsilon), t \rightarrow f(s, t)$ is a geodesic and in particular $f(0, t)=\gamma(t)$,

(2) $f(s, 0)=\alpha(s) \in L$ and $\frac{\partial f}{\partial t}(s, 0) \in \mathfrak{C}_{\alpha(s)}(L)$, for every $s \in(-\epsilon, \epsilon)$, where $\alpha$ is a smooth curve contained in $L$,

such that $J(t)=\frac{\partial f}{\partial s}(0, t)$ is a Jacobi field along $\gamma$ and $J(l)=0$. Observe that in particular we have

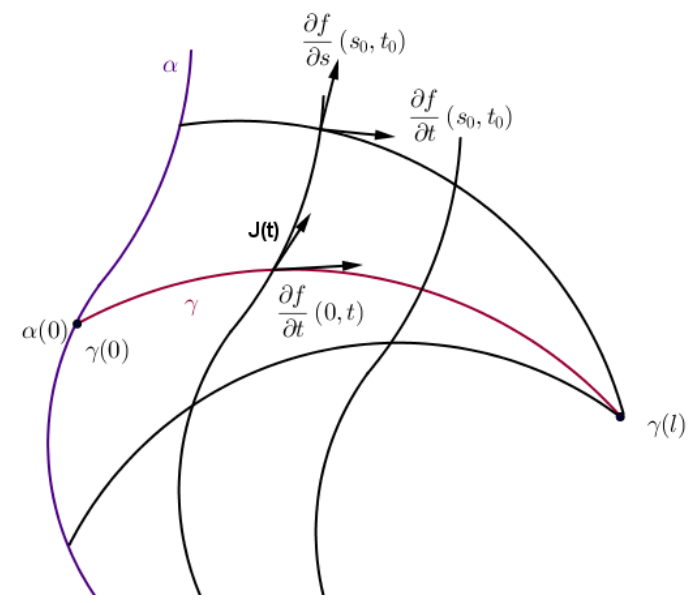

Figure 1.5

$\gamma(0)=\alpha(0)$ and $\gamma^{\prime}(0) \perp \alpha^{\prime}(0)$. For each $t \in[0, l]$, consider the curve $s \mapsto w(s)=\left(\alpha(s), t \frac{\partial f}{\partial t}(s, 0)\right)$ in $\mathfrak{C}(L)$ such that

$$
\exp ^{\perp}(w(s)):=\exp _{\alpha(s)}\left(t \frac{\partial f}{\partial t}(s, 0)\right)=f(s, t)
$$

Then for each $t \in[0, l]$,

$$
J(t)=\left.\frac{\partial}{\partial s} \exp ^{\perp}(w(s))\right|_{s=0}=d\left(\exp _{w(0)}^{\perp}\right) w^{\prime}(0)
$$

Then $J(l)=0$ if and only if $w^{\prime}(0) \in \operatorname{ker} d \exp _{w(0)}^{\perp}$ if and only if $w(0)$ is a critical point of $\exp ^{\perp}$ if and only if $\exp ^{\perp} w(0)=\exp _{\gamma(0)}\left(l \gamma^{\prime}(0)\right)=\gamma(l)=q$ is a critical value of $\exp ^{\perp}$ providing the assertion of Lemma. Note that we used the fact that $\exp ^{\perp}(p, v)=\exp _{p}(v)$.

Lemma 1.3.26 (Gauss). [BCS12] Fix $v \in T_{p} M \backslash 0$ and suppose that $\exp _{p}(v)$ is defined. Fix any instant $t \in[0,1]$ and take any vector $w \in T_{t v}\left(T_{p} M \backslash 0\right)$. Let $T(t)=d\left(\exp _{p}\right)_{t v} v$. Then

$$
g_{T(t)}\left(d\left(\exp _{p}\right)_{t v} w, T(t)\right)=g_{t v}(w, v) .
$$

Definition 1.3.27. Given a Finsler manifold $(M, F)$ and a connected immersed submanifold $L$ of codimension 1 of $M, L$ is called equifocal if for each parallel normal field $\xi$ on a neighborhood $U \subset L$, the derivative of the map $\eta_{\xi}: U \longrightarrow M$ defined by $\eta_{\xi}(x)=\exp _{x}(\xi)$ has constant rank. 


\subsubsection{Gradient and Transnormal Finsler functions}

Definition 1.3.28. For any smooth function $f:(M, F) \longrightarrow \mathbb{R}, \nabla f_{p}$ is called the gradient of $f$ at $p$ that is defined by

$$
d f_{p}(v)=g_{\nabla f_{p}}\left(\nabla f_{p}, v\right)
$$

where $v \in T_{p} M$.

Using Lemma 1.2.11, in the special case that $f=F$, one has the following lemma about the relation between $\nabla F(y)$ and $\frac{y}{F(y)}$ in a Minkowski space $(V, F)$, for every $y \in V$.

Lemma 1.3.29. Given a Minkowski space $(V, F)$, for every $0 \neq y \in V$,

$$
\nabla F(y)=\frac{y}{F(y)}
$$

Namely the gradient is a unitary vector that is in the direction of the position vector.

Proof. For every $u \in V$, with respect to Proposition 1.2.9 we have that

$$
g_{\frac{y}{F(y)}}\left(\frac{y}{F(y)}, u\right)=\left.\frac{1}{2} \frac{\partial}{\partial s} F^{2}\left(\frac{y}{F(y)}+s u\right)\right|_{s=0}=F\left(\frac{(y)}{F(y)}\right) \cdot d F(u)=\frac{F(y)}{F(y)} \cdot d F(u)=g_{\nabla F}(\nabla F, u) .
$$

Here we used the definition of the gradient of $F$ and the positive homogeneity of $F$. By Lemma 1.2.11, we conclude that

$$
\nabla F(y)=\frac{1}{F(y)} y
$$

Example 1.3.30. Consider the function $f: \mathbb{R}^{2} \longrightarrow \mathbb{R}$ by $y \longrightarrow f(y):=d_{F}(0, y)$ in the RandersMinkowski space $\left(\mathbb{R}^{2}, F\right)$ in which

$$
F(y)=\frac{2}{3}\left[\sqrt{3 y_{1}^{2}+4 y_{2}^{2}}-y_{2}\right]
$$

Let's find the gradient of $f$ at nonzero points. Observe that as in the Minkowski spaces the geodesics are the straight lines, for each $y$ the line $\gamma(t)=t y$ is the shortest curve joining the origin to $y$. So, with respect to $E Q .1 .3$, one can write

$$
f(y)=d_{F}(0, y)=F(y)=\frac{2}{3}\left[\sqrt{3 y_{1}^{2}+4 y_{2}^{2}}-y_{2}\right]
$$

Now according to Lemma 1.3.29 we have $\nabla f(y)=\nabla F(y)=\frac{y}{F(y)}$, for every $y \in \mathbb{R}^{2} \backslash 0$.

Proposition 1.3.31. [She01b] Given a Finsler space $(M, F)$, let $\mathcal{U}$ be an open subset of $M$ and $f$ be a smooth function on $\mathcal{U}$ with $d f \neq 0$. Put $\hat{g}:=g_{\nabla f}$ and $\hat{F}:=\sqrt{\hat{g}}$. Then

$$
\nabla f=\hat{\nabla} f
$$

where $\hat{\nabla} f$ denotes the gradient of $f$ with respect to $\hat{F}$. Moreover

$$
F(\nabla f)=\hat{F}(\hat{\nabla} f)
$$


In other words the gradients of $f$ with respect to both metrics $F$ and $\hat{F}$ coincides with the same norms.

Proof. According to the definition of gradient with respect to the metrics $F$ and $\hat{F}$ we have:

$$
\hat{g}(\hat{\nabla} f, w)=d f(w)=g_{\nabla f}(\nabla f, w)=\hat{g}(\nabla f, w), \forall w \in T \mathcal{U}
$$

Now as $\hat{g}$ is a Riemannian metric, equation (1.11) means that $\hat{\nabla} f=\nabla f$.

Now for the norms that is enough to replace $w=\hat{\nabla} f$ in (1.11). So

$$
\hat{F}(\hat{\nabla} f)=\sqrt{\hat{g}(\hat{\nabla} f, \hat{\nabla} f)}=\sqrt{\hat{g}(\nabla f, \nabla f)}=\sqrt{g_{\nabla f}(\nabla f, \nabla f)}=F(\nabla f)
$$

completing the proof.

Lemma 1.3.32. [She01b] Given a Finsler space $(M, F)$, let $\mathcal{U}$ be an open subset of $M$ and $f$ be a smooth function on $\mathcal{U}$ with $d f \neq 0$. Then $n:=\left.\frac{\nabla f}{F(\nabla f)}\right|_{f^{-1}(c)}$ is orthogonal to $f^{-1}(c)$ with respect to $g_{n}$, namely $n \in \mathfrak{C}\left(f^{-1}(c)\right)$.

Proof. As $\left.f\right|_{f^{-1}(c)}$ is constant, for very $w \in T f^{-1}(c)$ we have

$$
g_{n}(n, w)=\frac{1}{F(\nabla f)} g_{\nabla f}(\nabla f, w)=\frac{1}{F \nabla f} d f(w)=\left.\frac{1}{F \nabla f} \frac{d}{d t} f(\alpha(t))\right|_{t=0}=0,
$$

where $\alpha$ is a curve with $w=\alpha^{\prime}(0)$. Here we used the homogeneity of degree zero of the second fundamental form 1.2.9 and definition of the gradient 1.3.28.

Definition 1.3.33. Let $f:(M, F) \longrightarrow \mathbb{R}$ be a smooth function. If there exists a continuous function $\mathfrak{b}: f(M) \longrightarrow \mathbb{R}$ such that

$$
F^{2}(\nabla f)=\mathfrak{b} \circ f
$$

then $f$ is called a Finsler transnormal (shortly F-transnormal) function.

Note that according to this definition, the function $\mathfrak{b}$ is smooth on $f\left(M^{0}\right)$ where $M^{0}$ is the regular part of $M$. Let us recall the definition of transnormal in the case of Riemannian.

Definition 1.3.34. Let $f:(M, h) \longrightarrow \mathbb{R}$ be a smooth function, where $h$ is a Riemannian metric. If there exists a $C^{2}$ function $\mathfrak{b}: f(M) \longrightarrow \mathbb{R}$ such that

$$
\|\nabla f\|^{2}=\mathfrak{b} \circ f
$$

then $f$ is called a Riemannian transnormal (h-transnormal) function.

Remark 1.3.35. Considering these two definitions and Proposition 1.3.31, one can say that on the regular part $M^{0}, f$ is transnormal with respect to $F$ if and only if it is transnormal with respect to $\hat{g}$. 


\subsubsection{Morse-Bott functions and Transnormality}

Let $f: M \longrightarrow \mathbb{R}$ be a smooth function. Then $p \in M$ is a critical point of $f$ if $d f_{p}=0$.

Definition 1.3.36. [BH10] The Hessian of $f$ at a critical point $p$ is a symmetric linear form Hess $f_{p}$ such that for every $v, w \in T_{p} M$,

$$
\text { Hess } f_{p}(v, w)=V_{p}(W(f))
$$

where $V, W$ are the extensions of $v$ and $w$ to vector fields such that $V_{p}=v$ and $W_{p}=w$.

Let the critical set $C r(f)$ of $f$ contains a submanifold $C$. Put a Riemannian metric on $M$ and for every $p \in C$ consider the decomposition of $T_{p} M$ as

$$
T_{p} M=T_{p} C \oplus T_{p}^{\perp} C
$$

where $T_{p}^{\perp} C$ is the normal fiber. Let $v \in T_{p} C$ and $w \in T_{p} M$. Then

$$
\operatorname{Hess} f_{p}(v, w)=v_{p}(W(f))=0 \text {. }
$$

Since $v \in T_{p} C$, for any extension of $w$ to a vector field $W$ we have $\left.d f(W)\right|_{C}=0$. To show this, let $\gamma:(-\epsilon, \epsilon) \rightarrow C$ represent the tangent vector $v$. That is, $\gamma(0)=p$, and $\dot{\gamma}(0)=v$. Then we have

$$
W_{\gamma(t)}(f)=0
$$

for every $t \in(-\epsilon, \epsilon)$, as $\gamma(t)$ is a critical point. By the definition of the Hessian, it now follows immediately that $\operatorname{Hess} f_{p}(v, w)=0$. Hence the normal Hessian induces a symmetric bilinear form $\operatorname{Hess}^{\perp} f_{p}:=\left.\operatorname{Hess} f_{p}\right|_{T_{p}^{\perp} C}$ as

$$
\begin{aligned}
& \operatorname{Hess}^{\perp} f_{p}: T_{p}^{\perp} C \times T_{p}^{\perp} C \longrightarrow \mathbb{R} \\
&(v, w) \mapsto \operatorname{Hess} f_{p}(V, W) .
\end{aligned}
$$

Definition 1.3.37. A smooth function $f: M \longrightarrow \mathbb{R}$ is said to be Morse-Bott or a generalized Morse function if:

(i) the critical set $C r(f)$ is a finite disjoint union of connected submanifolds $C \subset C r(f)$;

(ii) the normal Hessian Hess ${ }^{\perp} f_{p}$ is non-degenerate, for every $p \in C$.

The index $\lambda_{p}$ of a critical point $p \in C$ is defined to be the maximal dimension of a subspace of $T_{p}^{\perp} C$ on which Hess ${ }^{\perp} f_{p}$ is negative definite. We mention a result from [QT15] that will be used in chapter 3 .

Theorem 1.3.38. Let $M$ be a compact smooth manifold, and $f: M \longrightarrow \mathbb{R}$ a Morse-Bott function with $C(f)=M_{+} \sqcup M_{-}$, where $M_{+}$and $M_{-}$are both close connected submanifolds of co-dimensions bigger than 1. Then there exists a Riemannian metric on $M$ so that $f$ is transnormal. In fact, the metric can be chosen so that $M_{+}$and $M_{-}$are both totally geodesics. 
Definition 1.3.39. Let $f:(M, F) \longrightarrow \mathbb{R}$ be a smooth function on a Finsler manifold $M$ and $\mathcal{U}=\left\{x \in M: \nabla f_{x} \neq 0\right\}$. We define Hess $f$ on $\mathcal{U}$ as

$$
\begin{aligned}
\text { Hess } f: \mathfrak{X}(\mathcal{U}) \times \mathfrak{X}(\mathcal{U}) \longrightarrow \mathbb{R} \\
(X, Y) \mapsto g_{\nabla f}\left(\nabla_{Y}^{\nabla f} \nabla f, X\right)
\end{aligned}
$$

Lemma 1.3.40. Let $f:(M, F) \longrightarrow \mathbb{R}$ be a smooth function on a Finsler manifold $M$ and $\mathcal{U}=$ $\left\{x \in M: \nabla f_{x} \neq 0\right\}$. Then

$$
\text { Hess } f(X, Y)=X(Y(f))-d f\left(\nabla_{Y}^{\nabla f} X\right)
$$

Proof. By the almost $g$-compatibility of the connection and the definition of the gradient we have

$$
\begin{array}{r}
g_{\nabla f}\left(\nabla_{Y}^{\nabla f} \nabla f, X\right)+g_{\nabla f}\left(\nabla_{Y}^{\nabla f} X, \nabla f\right)+2 C_{\nabla f}\left(\nabla_{Y}^{\nabla f} \nabla f, \nabla f, X\right)= \\
Y\left(g_{\nabla f}(\nabla f, X)\right)=Y(d f X)=Y(X(f)) .
\end{array}
$$

So according to item $(c)$ of Lemma 1.3.3 and the definition of the gradient we have

$$
g_{\nabla f}\left(\nabla_{Y}^{\nabla f} \nabla f, X\right)=Y(X(f))-g_{\nabla f}\left(\nabla_{Y}^{\nabla f} X, \nabla f\right)=X(Y(f))-d f\left(\nabla_{Y}^{\nabla f} X\right) .
$$

In this Lemma, as $\nabla$ is homogeneous of degree zero, one can write Hess $f(X, Y)_{\gamma(t)}=X(Y(f))_{\gamma(t)}-$ $d f\left(\nabla_{Y}^{\gamma^{\prime}(t)} X\right)_{\gamma^{\prime}(t)}$, where $\gamma$ is the integral curve of $\frac{\nabla f}{\|\nabla f\|}$ and we know that it can be extended to the critical level sets. That is $\gamma(0) \in f^{-1}(f(q))$, for the critical point $q$. Now one has

$$
\operatorname{Hess} f(X, Y)_{q}=\lim _{t \mapsto 0} \operatorname{Hess} f(X, Y)_{\gamma(t)}=\lim _{t \mapsto 0}\left[X(Y(f))_{\gamma(t)}-d f\left(\nabla_{Y}^{\gamma^{\prime}(t)} X\right)\right] \text {. }
$$

As in the critical points $d f=0$, the second term above vanishes and so we have Definition 1.3.36. Indeed in the critical points the metric does not play any role and the Hessian is just a topological object.

Lemma 1.3.41. Let $f:(M, F) \longrightarrow \mathbb{R}$ be a smooth function which is $F$-transnormal with $F^{2}(\nabla f)=$ $\mathfrak{b} \circ f$ and $\mathcal{U}=\left\{x \in M: \nabla f_{x} \neq 0\right\}$. Then on $\mathcal{U}$ we have

$$
\text { Hess } f(\nabla f, \nabla f)=\frac{1}{2} \mathfrak{b}^{\prime}(f) \mathfrak{b}(f) .
$$

In particular, one can write Hess $f\left(\frac{\nabla f}{F(\nabla f)}, \frac{\nabla f}{F(\nabla f)}\right)=\frac{1}{2} \mathfrak{b}^{\prime}(f)$.

Proof. According to Definition 1.3.39 and the hypothesis we have

$$
\text { Hess } \begin{aligned}
f(\nabla f, \nabla f) & =g_{\nabla f}\left(\nabla_{\nabla f}^{\nabla f} \nabla f, \nabla f\right)=\frac{1}{2} \nabla f g_{\nabla f}(\nabla f, \nabla f) \\
& =\frac{\nabla f}{2}\left(F^{2}(\nabla f)\right)=\frac{\nabla f}{2}(\mathfrak{b} \circ f)=\frac{1}{2} d(\mathfrak{b} \circ f)(\nabla f) \\
& =\frac{1}{2} \mathfrak{b}^{\prime}(f) d f(\nabla f)=\frac{1}{2} \mathfrak{b}^{\prime}(f) g_{\nabla f}(\nabla f, \nabla f) \\
& =\frac{1}{2} \mathfrak{b}^{\prime}(f) F^{2}(\nabla f)=\frac{1}{2} \mathfrak{b}^{\prime}(f) \mathfrak{b}(f) .
\end{aligned}
$$


In above calculations, in the second equality, we used the almost $g$-compatibility of the Chern connection as well as item $(c)$ of Lemma 1.3.3. In the next equality we used the equality $F^{2}(\nabla f)=$ $g_{\nabla f}(\nabla f, \nabla f)$ that comes from Proposition 1.2.9. In order to prove the last assertion observe that

$$
\operatorname{Hess} f\left(\frac{\nabla f}{F(\nabla f)}, \frac{\nabla f}{F(\nabla f)}\right)=\frac{1}{F^{2}(\nabla f)} \operatorname{Hess} f(\nabla f, \nabla f)=\frac{1}{\mathfrak{b}(f)} \operatorname{Hess} f(\nabla f, \nabla f)=\frac{1}{2} \mathfrak{b}^{\prime}(f) .
$$

Definition 1.3.42. Given a vector space $V$, the kernel of a symmetric bilinear form $Q: V \times V \longrightarrow$ $\mathbb{R}$ is the set

$$
\operatorname{ker}(Q)=\{v \in V \mid Q(v, w)=0 \text { for every } w \in V\}
$$

Also equivalently one can write

$$
\operatorname{ker}(Q)=\left\{v \in V \mid Q\left(v, u_{i}\right)=0\right\}
$$

where $u_{i} \cdots, u_{n}$ are the basis vectors.

Observe that given a manifold $M$ and a smooth function $f: M \longrightarrow \mathbb{R}$ the Hessian matrix $H=D^{2} f(q)$, where $q$ is a critical point, is symmetric and so it is characterized by its eigenvalues and eigenvectors. In fact we have the following theorem.

Theorem 1.3.43 (Spectral Theorem). Assume that $H$ is a symmetric matrix. Then:

a. $H$ has all real eigenvalues $\lambda_{i} \in \mathbb{R}$, for $i=1, \ldots, n$;

b. there exists an orthonormal basis for $\mathbb{R}^{n}$ (and so for $T_{q} M$ ) composed of eigenvectors of $H$;

c. the matrix $H$ is diagonal when expressed in the basis of eigenvectors. More specifically, if $U$ is the matrix whose columns are the eigenvectors $u_{i}$, then $H U=U \wedge$ where $\wedge=\operatorname{diag}\left(\lambda_{1}, \ldots, \lambda_{n}\right)$.

According to item $(c)$ of Spectral Theorem we have that $H u_{i}=\lambda_{i} u_{i}$ and so $u_{i}^{T} H u_{i}=\lambda_{i}$. observe that for every vector $v$, if we express $v$ in terms of he eigenvectors $v=\sum_{i=1}^{n} c_{i} u_{i}, c_{i} \in \mathbb{R}$ then we have

$$
v^{T} H v=\sum_{i, j=1}^{n} c_{i} c_{j} u_{i}^{T} H u_{i}=\sum_{i=1}^{n} c_{i}^{2} \lambda_{i} .
$$

Note that the second derivative in a specific direction represented by a unit vector $u_{i}$ is given by $u_{i}^{T} H u_{i}$ which is indeed equal to $\lambda_{i}$. Because, actually, we have

$$
\text { Hess } f_{q}(X, Y)=X_{q}(Y(f))=\sum_{i, j=1}^{n} X_{i} Y_{j} \frac{\partial^{2} f}{\partial x^{i} \partial x^{j}}(q)=X_{q}^{T} H Y_{q} \text {, }
$$

where $\left(x^{1}, \ldots, x^{n}\right)$ is the coordinate system.

Lemma 1.3.44. Given a manifold $M$ of dimension $n$, assume that $f: M \longrightarrow \mathbb{R}$ is a smooth function and $b \in \mathbb{R}$ a critical value of it. Also we assume that $f^{-1}(b)$ is an embedded submanifold of $M$. Then for every $q \in f^{-1}(b)$, 
a. $T_{q} f^{-1}(b) \subset$ ker Hess $f_{q}$, that is for every $v \in T_{q} f^{-1}(b)$ one has Hess $f_{q}(v, w)=0$, for every $w \in T_{q} M$;

b. one can choose coordinates $\left(x^{1}, \ldots, x^{k}, y^{1}, \cdots, y^{l}\right): U \subset M \longrightarrow \mathbb{R}^{n}$, where $l+k=n$ and $l=\operatorname{dim} f^{-1}(b)$, on some neighborhood $U$ of $q$ such that

$$
\text { Hess } f_{q}=\left(\begin{array}{cccccc}
\frac{\partial^{2} f}{\partial x_{1}^{2}} & \cdots & \frac{\partial^{2} f}{\partial x_{1} \partial x_{k}} & 0 & \cdots & 0 \\
\vdots & \ddots & \vdots & \vdots & \ddots & \vdots \\
\frac{\partial^{2} f}{\partial x_{1} \partial x_{k}} & \cdots & \frac{\partial^{2} f}{\partial x_{k}^{2}} & 0 & \cdots & 0 \\
0 & \cdots & 0 & 0 & \cdots & 0 \\
\vdots & \ddots & \vdots & \vdots & \ddots & \vdots \\
0 & \cdots & 0 & 0 & \cdots & 0
\end{array}\right) \text {. }
$$

Proof. In order to prove item (a), by Definition 1.3.42, it is sufficient to show that for every $v \in T_{q} f^{-1}(b)$, Hess $f_{q}(v, w)=0$, for every $w \in T_{q} M$. So given $v \in T_{q} f^{-1}(b)$ and $w \in T_{q} M$, let $\alpha:(-\epsilon, \epsilon) \longrightarrow f^{-1}(b)$ represent the integral curve of $v$ and $W$ be any extension of $w$ to a neighborhood of $q$. Then we have

$$
W_{\alpha(t)}(f)=d f_{\alpha(t)}(W)=0
$$

for every $t \in(-\epsilon, \epsilon)$, as $\alpha(t)$ is a critical point. By Definition 1.3.36, it now follows immediately that Hess $f_{p}(v, w)=0$ which implies that $v \in \operatorname{ker} \operatorname{Hess} f_{q}$. Observe that from equality $\operatorname{Hess} f_{p}(v, w)=0$, for every $w \in T_{q} M$, in particular we have that

$$
\text { Hess } f_{p}(v, w)=0
$$

for every $w \in T_{q} f^{-1}(b)$.

To prove item $(b)$, as $f^{-1}(b)$ is an embedded submanifold, one can choose coordinates $\left(x^{1}, \cdots, x^{k}, y^{1}, \cdots, y^{l}\right): U \subset M \longrightarrow \mathbb{R}^{n}$ on some neighborhood $U \ni q$ such that $U \cap f^{-1}(b)=$ $\{(x, y) \in U \mid x=0\}$. According to the fact that Hess $f_{q}(v, w)=0$, for every $w \in T_{q} f^{-1}(b)$, we have that

$$
\frac{\partial^{2} f}{\partial y^{i} \partial y^{j}}=0
$$

$i, j=1, \cdots, l$. Moreover from Hess $f_{q}(v, w)=0$, for every $v \in T_{q} f^{-1}(b)$ and $w \in T_{q}^{\perp} f^{-1}(b)$ we have

$$
\frac{\partial^{2} f}{\partial x^{i} \partial y^{j}}=0
$$

$i=1, \cdots, k, j=1, \cdots, l$. Here we are considering the decomposition

$$
T_{q} M=T_{q} f^{-1}(b) \oplus T_{q}^{\perp} f^{-1}(b),
$$


for a given Riemannian metric $h$. To sum up,

$$
\operatorname{Hess} f_{q}=\left(\begin{array}{cccccc}
\frac{\partial^{2} f}{\partial x_{1}^{2}} & \cdots & \frac{\partial^{2} f}{\partial x_{1} \partial x_{k}} & 0 & \cdots & 0 \\
\vdots & \ddots & \vdots & \vdots & \ddots & \vdots \\
\frac{\partial^{2} f}{\partial x_{1} \partial x_{k}} & \cdots & \frac{\partial^{2} f}{\partial x_{k}^{2}} & 0 & \cdots & 0 \\
0 & \cdots & 0 & 0 & \cdots & 0 \\
\vdots & \ddots & \vdots & \vdots & \ddots & \vdots \\
0 & \cdots & 0 & 0 & \cdots & 0
\end{array}\right)_{n \times n}
$$

Observe that this matrix has at least $l$ eigenvalues equal to 0 .

Remark 1.3.45. This is necessary to emphasize that $f^{-1}(b)$ is an embedded submanifold. For example consider $f: \mathbb{R}^{2} \longrightarrow \mathbb{R}, f(x, y)=x y$. The origin of $\mathbb{R}^{2}$ is a critical point for $f$. However the level set $f^{-1}\{0\}$ is not a submanifold in any neighborhood of the origin, due to self-intersection. We could consider the submanifold $N=\left\{(x, y) \in \mathbb{R}^{2}: y=0\right\}$, but observe that

$$
\operatorname{Hess}_{(0,0)} f((1,0),(0,1))=1 \neq 0
$$

even if $(1,0) \in T_{(0,0)} N$.

\subsection{Submersion}

Let us in this section remind and introduce the concept of Riemannian [BCO16] and Finsler [ÁPD01] submersions.

Definition 1.4.1. Let $M$ and $N$ be Riemannian manifolds. A smooth surjective map $\pi: M \longrightarrow N$ is called a Riemannian submersion if $d \pi_{p}:\left(\operatorname{ker} d \pi_{p}\right)^{\perp} \longrightarrow T_{\pi(p)} N$ is a linear isometry, for all $p \in M$.

The distribution $\nu$ on $M$ defined by $q \longrightarrow \operatorname{ker}\left(d \pi_{q}\right)$ is called vertical distribution and its orthogonal complement $\mathcal{H}$ is called horizontal distribution. Indeed at each point $p \in M$ one can write

$$
T_{p} M=\nu_{p} \oplus \mathcal{H}_{p}
$$

The vertical distribution is always integrable. Because any leaf of the vertical distribution is a connected component of the fiber, i.e., the preimage by $\pi$ of an element in $N$. Let $\alpha: I \longrightarrow N$ be a smooth curve. A curve $\tilde{\alpha}: I \longrightarrow M$ is called horizontal lift of $\alpha$ if $\pi(\tilde{\alpha})=\alpha$ and $\tilde{\alpha}$ is a horizontal curve, i.e. $\tilde{\alpha}^{\prime}(t) \in \mathcal{H}_{\tilde{\alpha}(t)}$, for every $t \in I$. If $\alpha: I \longrightarrow M, t_{0} \in I$ and $q \in \pi^{-1}\left(\alpha\left(t_{0}\right)\right)$, then there exists a unique horizontal lift $\tilde{\alpha}: I \longrightarrow M$ of $\alpha$ defined on a neighborhood of $t_{0}$ such that $\tilde{\alpha}\left(t_{0}\right)=q$.

Definition 1.4.2. Given a manifold $M$, a smooth vector field $X \in \mathfrak{X}(M)$ is called projectable if it is $\pi$ related to some vector field $Y \in \mathfrak{X}(N)$, i.e.

$$
d \pi_{p}(X)=Y(\pi(p)),
$$


for all $p \in M$. It is standard to show that $X$ is projectable if and only if

$$
d \pi_{p}(X)=d \pi_{q}(X)
$$

when $\pi(p)=\pi(q)$.

Definition 1.4.3. A surjective linear map $\pi: V_{1} \longrightarrow V_{2}$ between two normed spaces $\left(V_{1},\|\cdot\|_{1}\right)$ and $\left(V_{2},\|.\|_{2}\right)$ is said to be an isometric submersion if the image of the closed ball on $V_{1}$ under the map $\pi$ equals the closed unit ball on $V_{2}$.

Definition 1.4.4. A submersion $\pi: M \longrightarrow N$ between Finsler manifolds is said to be isometric if for every point $p \in M$ the differential $d \pi_{p}: T_{p} M \longrightarrow T_{\pi(p)} N$ is an isometric submersion of Minkowski spaces.

\subsubsection{Singular Finsler foliation}

In this section we want to talk about singular Finsler foliation (for short SFF). To make a comparison with, we first state the singular Riemannian foliation (for short SRF) [AJ11] and then carry on with the definition of the Finsler case.

Definition 1.4.5. Given a manifold $M$, let $\mathcal{F}$ be a partition of $M$ into smooth, complete, and injectively immersed submanifolds. Each such a submanifold is called a leaf and $\mathcal{F}$ is called a foliation of $M$. We denote a leaf through $p$ with $L_{p} . \mathcal{F}$ is called a singular Riemannian foliation (SRF) if:

(i) it is Singular, i.e. for every $v \in T_{p} L_{p}$ there exists $X \in \mathfrak{X}(U)$ in which $U$ is an open neighborhood of $p$ such that $X_{p}=v$ and $X_{q} \in T_{q} L_{q}$, for every $q \in U$;

(ii) it is Transnormal, i.e. for each geodesic $\gamma:(-\epsilon, \epsilon) \longrightarrow M$ such that $\gamma^{\prime}(0) \in \mathcal{H}_{\gamma(0)}$ we have $\gamma^{\prime}(t) \in \mathcal{H}_{\gamma(t)}$. That is if a geodesic is perpendicular to a leaf, it remains perpendicular to all the leaf it meets.

The tangent to a leaf at the point $p$ is called the vertical space which is $\nu_{p}=T_{p} L_{p}$. Once we have a Riemannian space $(M, h)$ we have the decomposition

$$
T_{p} M=\nu_{p} \oplus \mathcal{H}_{p}
$$

where $\mathcal{H}_{p}=\left(T_{p} L_{p}\right)^{\perp}$ and it is called the horizontal space (indeed orthogonal to the leaf with respect to $h$ ).

Definition 1.4.6. Let $\mathcal{F}$ be a SRF. Then a leaf L (and each point of it) is called regular if its dimension is maximal; otherwise it is called singular. The co-dimension of a SRF is defined as the co-dimension of its regular leaves.

Now, in the case of a Finsler space $(M, F)$ in order to define the singular Finsler foliation, first remember that we say that $v \in T_{p} M$ is orthogonal to a leaf $L_{p}$ (see 1.3.1) if it belongs to the cone $\mathfrak{C}_{p}\left(L_{p}\right)$. Observe that the vertical space $\nu_{p}$ and the leaf $L_{p}$ are both geometric objects which are not dependent on the metric, however $\mathcal{H}_{p}$ does. In the sequel the definition of the singular Finsler foliation is given. 
Definition 1.4.7. Given a Finsler space $(M, F)$ with a singular foliation $\mathcal{F}$, the triple $(M, F, \mathcal{F})$ is called a singular Finsler foliation if for a geodesic $\gamma:(-\epsilon, \epsilon) \longrightarrow M$ with $\gamma^{\prime}(0) \in \mathfrak{C}_{\gamma(0)}\left(L_{\gamma(0)}\right)$ we have that

$$
\gamma^{\prime}(t) \in \mathfrak{C}_{\gamma(t)}\left(L_{\gamma(t)}\right)
$$

for every $t$.

It is equivalent to saying that if $\gamma^{\prime}(0)$ is orthogonal to the leaf $L_{\gamma(0)}$ at the direction of itself then $\gamma^{\prime}(t)$ for every $t$ is orthogonal to the leaf $L_{\gamma(t)}$ at the direction of itself.

Example 1.4.8. Consider the Randers-Minkowski space $\left(\mathbb{R}^{2}, F\right)$ in which

$$
F(y)=\frac{2}{3}\left[\sqrt{3 y_{1}^{2}+4 y_{2}^{2}}-y_{2}\right]
$$

with the vertical projection $\pi: \mathbb{R}^{2} \longrightarrow \mathbb{R}$ with $\pi\left(y_{1}, y_{2}\right)=y_{1}$. Here in following picture two geodesics passing through the leaf $L=\pi^{-1}(0)$ are shown. It is not difficult to see that

$$
\mathcal{F}=\left\{\pi^{-1}(t)\right\}_{t \in \mathbb{R}}
$$

is a SFF. In fact, first notice that in Minkowski space the geodesics are straight lines. In the next chapter we will see that for a unit speed geodesic $\gamma$ we have

$$
\gamma^{\prime}(t) \in \mathfrak{C}_{\gamma(t)} \text { if and only if } \gamma^{\prime}(t)-W \in \mathcal{H}_{\gamma(t)}
$$

where $\mathcal{H}_{\gamma(t)}$ is the horizontal space with respect to the Euclidean metric. In our case we have that

$$
\gamma^{\prime}(0)-W \in \mathcal{H}_{\gamma(0)} \text { if and only if } \gamma^{\prime}(t)-W \in \mathcal{H}_{\gamma(t)},
$$

for every $t$. It guarantees that if $\gamma$ is orthogonal to a leaf at some point, it remains orthogonal to all the leaves it meets.

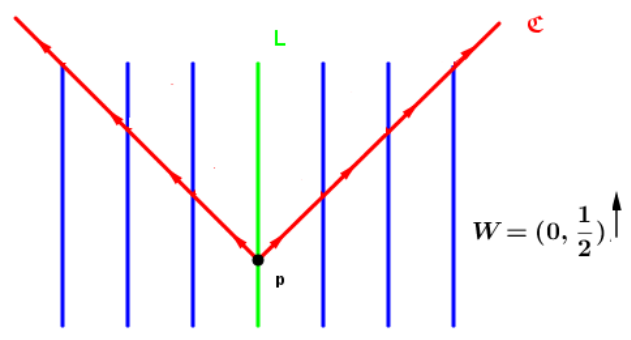

Figure 1.6: Cone

In the next chapter Example 2.4.1 is provided which is a non Finslerian foliation.

\subsubsection{Parallelism}

Definition 1.4.9. Given a Finsler space $(M, F)$ and closed subsets $C$ and $D$ of it we say that $C$ is parallel to $D$ if for every $x, y \in C$ we have

$$
d_{F}(x, D)=d_{F}(y, D)
$$


To simplify, by $C \| D$ we mean $C$ is parallel to $D$ and by $C \nVdash D$ we mean $C$ is not parallel to $D$. Observe that, equivalently, one can check if for every $x, y \in D$ the equality $d_{F}(C, x)=d_{F}(C, y)$ satisfies or not.
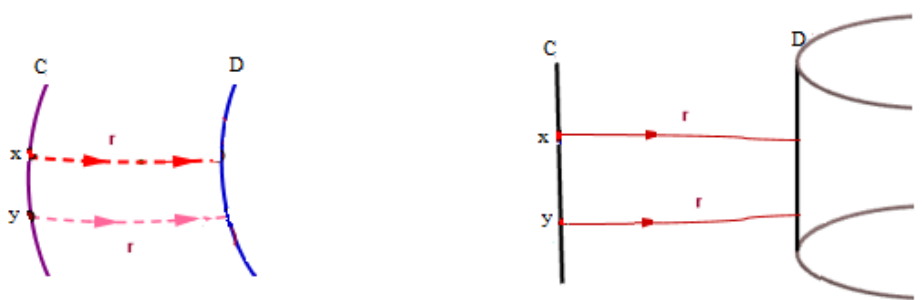

Figure 1.7

Remark 1.4.10. Note that if for every $x \in C, d_{F}(x, D)=r$ we simply write $d_{F}(C, D)=r$. Also in this definition $C \| D$ does not imply that $D \| C$ (It can be seen in the following example.). Even though both $C$ and $D$ are parallel to each other it does not mean that the distances must be equal.

Definition 1.4.11. We say that $C$ is equidistant to $D$ if $C \| D$ and $D \| C$ in which the distances may not be the same.

Example 1.4.12. Consider the Randers-Minkowski space $\left(\mathbb{R}^{2}, F\right)$ in which

$$
F(y)=\frac{2}{3}\left[\sqrt{3 y_{1}^{2}+4 y_{2}^{2}}-y_{2}\right]
$$

and the indicatrices $\Sigma^{F}$ and $\Sigma_{2}^{F}$ where

$$
\Sigma_{r}^{F}:=\left\{y \in \mathbb{R}^{2} \mid d_{F}(0, y)=r\right\}
$$

and $\Sigma^{F}:=\Sigma_{1}^{F}$. According to Lemmas 1.3.32 and 1.3.29, for every $y \in \Sigma^{F}$ we have that $y \in \mathfrak{C}_{p}\left(\Sigma^{F}\right)$. Note that in fact $\Sigma_{r}^{F}=F^{-1}(r)$ or in other words each indicatrix is a leaf associated to the function $F$. Since in the Minkowski spaces the geodesics are straight lines, each straight line $\gamma(t)=y t$ is a geodesic such that $\gamma^{\prime}(1) \in \mathfrak{C}_{y}\left(\Sigma^{F}\right)$ and $\gamma^{\prime}(2) \in \mathfrak{C}_{\gamma(2)}\left(\Sigma_{2}^{F}\right)$. Now by Lemma 1.3.13, $\gamma$ minimizes the distance from $y$ to $\Sigma_{2}^{F}$ which means

$$
d_{F}\left(y, \Sigma_{2}^{F}\right)=L\left(\left.\gamma\right|_{[1,2]}\right)=\int_{1}^{2} F\left(\gamma^{\prime}\right) d t=\int_{1}^{2} d t=1
$$

Because for every $y \in \Sigma^{F}$ we have the same distance, it is concluded that

$$
\Sigma^{F} \| \Sigma_{2}^{F}
$$

See Picture 1.8. Now let's calculate $d_{F}\left(y, \Sigma^{F}\right)$ for two special points $y=(0,-1)$ and $y=(0,3)$ which are in $\Sigma_{2}^{F}$. Notice that according to Table 2.2 the geodesics $\gamma_{1}(t)=t\left(0, \frac{1}{2}\right)$ and $\gamma_{2}(t)=t\left(0,-\frac{1}{2}\right)$ are orthogonal to the leaf $\Sigma_{1}^{F}$, at each point they meet this leaf (Note that each intersects the leaf at exactly two points.). So according to Lemma 1.3.13, $\gamma_{1}$ minimizes the distance from $\gamma_{1}(-2)=$ 
$(0,-1) \in \Sigma_{2}^{F}$ to $\Sigma^{F}$. Hence

$$
d_{F}\left((0,-1), \Sigma^{F}\right)=L\left(\left.\gamma_{1}\right|_{[-2,-1]}\right)=\int_{-2}^{-1} F\left(\gamma_{1}^{\prime}(t)\right) d t=\int_{-2}^{-1} \frac{1}{3} d t=\frac{1}{3} .
$$

Also, by the same Lemma (1.3.13), $\gamma_{2}$ minimizes the distance from $\gamma_{2}(-6)=(0,3) \in \Sigma_{2}^{F}$ to $\Sigma^{F}$. Therefore

$$
d_{F}\left((0,3), \Sigma^{F}\right)=L\left(\left.\gamma_{2}\right|_{[-6,-3]}\right)=\int_{-6}^{-3} F\left(\gamma_{2}^{\prime}(t)\right) d t=\int_{-6}^{-3} d t=3 .
$$

By Definition 3.3.4, it means

$$
\Sigma_{2}^{F} \nmid \Sigma^{F}
$$

Indeed this metric comes from an Euclidean metric that is changed under the influence of the wind $W=\left(0, \frac{1}{2}\right)$. As it could be foreseen the movement at the direction of the wind makes the movement faster than a movement that makes a non zero angle with the wind. Specially moving along a direction which makes the angle $\pi$ with the wind (i.e. at the opposite direction of the wind) takes longer.

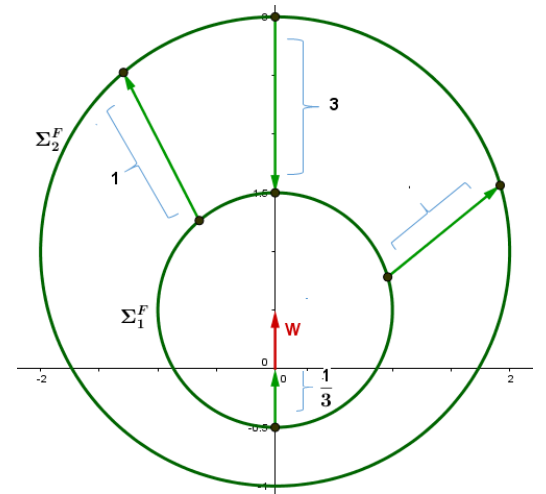

Figure 1.8: Example 1.4.12

\subsubsection{Tubular neighborhoods and geometric cylinders}

Let $P$ be a precompact (a subset whose closure is compact) submanifold of a Finsler manifold $(M, F)$; for example $P$ could be a plaque (i.e. it is relatively compact) of a singular foliation. Then there exists $r>0$ such that the exponential map from

$$
\mathfrak{C}(P) \cap B(r):=\cup_{p \in P} \mathfrak{C}_{p} \cap B_{p}(r)
$$

into its image is a diffeomorphism. Here $B_{p}(r)$ is the ball of radius $r$ centered at $p$. This image is called a tubular neighborhood of $P$ [AJ17]. One of the benefits of existence of such a neighborhood is that for each point contained in a tubular neighborhood of $P$, there exists a unique minimizing geodesic from $P$ to that point. We also will show that the geometric cylinders which are contained in tubular neighborhoods are not degenerated. we give the formal definitions as below.

Definition 1.4.13. Given a precompact submanifold $P$ of $M$, the future tubular neighborhood of $P$ is an open subset as

$T u b_{r}^{+}(P):=\left\{\gamma(s) \mid \gamma:[0, r] \longrightarrow M\right.$ is a unique minimizing geodesic with $\left.\gamma^{\prime}(0) \in \mathfrak{C}_{\gamma(0)}(P) \cap \Sigma^{F}\right\}$, 
similarly one can define the past tubular neighborhood as follows:

$T u b_{r}^{-}(P):=\left\{\gamma(s) \mid \gamma:[0, r] \longrightarrow M\right.$ is a unique minimizing geodesic with $\left.\gamma^{\prime}(r) \in \mathfrak{C}_{\gamma(r)}(P) \cap \Sigma^{F}\right\}$.

Here $s \in(0, r)$.

Definition 1.4.14. Given a precompact submanifold $P$ of a Finsler manifold $(M, F)$, a future (geometric) cylinder of radius $r$ and the axis $P$ (as it is expected) is defined as

$$
C_{r}^{+}(P):=\left\{x \in M: d_{F}(P, x)=r\right\} .
$$

By an analogue way the past (geometric) cylinder of radius $r>0$ and the axis $P$ is defined as

$$
C_{r}^{-}(P):=\left\{x \in M: d_{F}(x, P)=r\right\} .
$$

Here, to get some intuition, the past and future cylinders for the cases in which $P$ is a 1dimensional submanifold Figure 1.9 of a manifold $M$ of dimension 3 and a 2-dimensional submanifold Figure 1.10 of a manifold $M$ of dimension 3 are given.

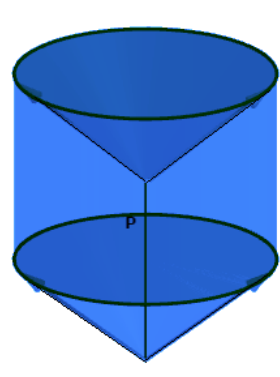

$C_{r}^{+}(P)$

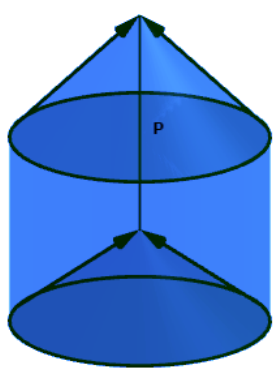

$C_{r}^{-}(P)$

Figure 1.9: Future and past cylinders

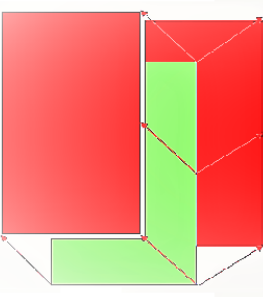

$C_{r}^{+}(P$

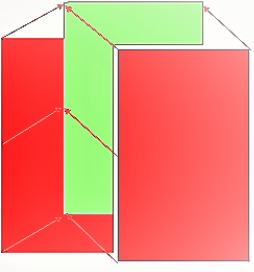

$C_{r}^{-}(P)$

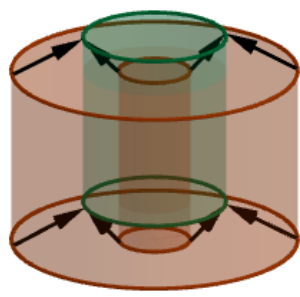

$C_{r}^{-}(P)$

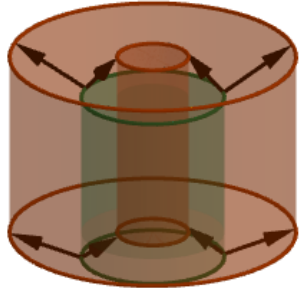

$C_{r}^{+}(P)$

Figure 1.10: Future and past cylinders

Definition 1.4.15. [She01b] For a given compact subset $A \subset M$, we define $\rho_{+}: M \longrightarrow \mathbb{R}$ with $\rho_{+}(x)=d_{F}(A, x)$ and $\rho_{-}: M \longrightarrow \mathbb{R}$ with $\rho_{-}(x)=d_{F}(x, A)$.

Remark 1.4.16. For $\rho_{+}$and for every $x, y \in M$ we have the following relation

$$
-d_{F}(y, x) \leq \rho_{+}(y)-\rho_{+}(x) \leq d_{F}(x, y) .
$$

There is the similar relation for $\rho_{-}$. 
Remark 1.4.17. $\rho_{+}$and $\rho_{-}$are locally Lipschitz continuous on $M$ and so they are almost differentiable everywhere.

Lemma 1.4.18. For a Finsler space $(M, F)$ with $\rho_{+}$and $\rho_{-}$defined as 1.4.15, if for every $p, q \in M$ there exists a shortest unit speed curve from $p$ to $q$, then

$$
F\left(\nabla \rho_{-}\right)=1=F\left(\nabla \rho_{+}\right)
$$

Remark 1.4.19. With the assumptions of this lemma $\rho_{+}$and $\rho_{-}$are transnormal with respect to F with

$$
\mathfrak{b}_{+} \equiv 1 \equiv \mathfrak{b}_{-},
$$

where $\mathfrak{b}_{+}: \mathbb{R}^{+} \longrightarrow \mathbb{R}$ and $\mathfrak{b}_{-}: \mathbb{R}^{-} \longrightarrow \mathbb{R}$

Lemma 1.4.20. Given a precompact submanifold $P \subset M$, consider the map $\rho_{+}: U \backslash P \longrightarrow \mathbb{R}$ with $\rho_{+}(x)=d_{F}(P, x)$, where $U:=T u b_{r}^{+}(P)$ for some $r>0$. Then the followings hold:

(1) $\rho_{+}$is smooth

(2) for each point $x \in U$ there exists a unique unit speed minimizing geodesic $\gamma$ such that

$$
\begin{aligned}
& -\gamma^{\prime}(0) \in \mathfrak{C}_{\gamma(0)}(P) \\
& -\gamma(s)=x, \text { for some } s<r \\
& -L(\gamma)=\rho_{+}(x)=s
\end{aligned}
$$

(3) there exits a neighborhood $W$ in $\mathfrak{C}(P)=\cup_{p \in P}\left\{\mathfrak{C}_{p} P \backslash 0\right\}$ such that $\exp ^{\perp}: W \backslash P \longrightarrow U \backslash P$ is a diffeomorphism.

Corollary 1.4.21. In Lemma 1.4.20, if for some neighborhood $U$ of $P, \rho_{+}$is smooth, then the items (2) and (3) hold.

Lemma 1.4.22. There exists a smooth canonical projection $\pi: U \backslash P \longrightarrow P$ with $\pi(x)=\gamma(0)$, where $\gamma$ is the geodesic in the second item of Lemma 1.4.20 and $U=T u b_{r}^{+}(P)$.

Lemma 1.4.23. Given a precompact submanifold $P$ of $M$, let $T u b_{r}^{+}(P)$ and $T u b_{r}^{-}(P)$ be the future and past tubular neighborhoods, respectively, of $P$. Then for every $s \in(0, r]$,

(a) $C_{s}^{+}(P)=\left\{\gamma(s) \mid \gamma:[0, r] \longrightarrow M\right.$ is a unique minimizing geodesic with $\left.\gamma^{\prime}(0) \in \mathfrak{C}_{\gamma(0)}^{1}(P)\right\}$;

(b) $C_{s}^{-}(P)=\left\{\gamma(0) \mid \gamma:[0, r] \longrightarrow M\right.$ is a unique minimizing geodesic with $\left.\gamma^{\prime}(s) \in \mathfrak{C}_{\gamma(s)}^{1}(P)\right\}$;

(c) $C_{s}^{+}(P)$ and $C_{s}^{-}(P)$ are closed and embedded submanifolds each of codimension 1;

(d) If $\gamma$ is a unitary speed geodesic with $\gamma^{\prime}(0) \in \mathfrak{C}_{\gamma(0)}^{1}(P)$, then $\gamma^{\prime}(t) \in \mathfrak{C}_{\gamma(t)}\left(C_{t}^{+}(P)\right)$;

(e) If $\gamma$ is a unitary speed geodesic with $\gamma^{\prime}(0) \in \mathfrak{C}_{\gamma(0)}^{1}\left(C_{t}^{-}(P)\right)$, then $\gamma^{\prime}(t) \in \mathfrak{C}_{\gamma(t)}(P)$;

(f) If $\gamma$ is a geodesic with $\gamma^{\prime}(0) \in \mathfrak{C}_{\gamma(0)}^{1}(P)$ and $x=\gamma(s) \in C_{s}^{+}(P)$, then

$$
T_{x} C_{s}^{+}(P)=\left\{v \in T_{x} M: g_{\gamma^{\prime}(s)}\left(\gamma^{\prime}(s), v\right)=0\right\} ;
$$


(g) If $\gamma$ is a geodesic with $\gamma^{\prime}(0) \in \mathfrak{C}_{\gamma(0)}^{1}(P)$ and $x=\gamma(-s) \in C_{s}^{-}(P)$, then

$$
T_{x} C_{s}^{-}(P)=\left\{v \in T_{x} M: g_{\gamma^{\prime}(-s)}\left(\gamma^{\prime}(-s), v\right)=0\right\} .
$$

Proof. To prove (a), first notice that according to the definition of the future cylinder 1.4.14,

$$
C_{s}^{+}(P) \subset \overline{T u b_{r}^{+}(P)}
$$

So for every $x \in C_{s}^{+}(P)$ there exists a unique unit speed minimizing geodesic $\gamma$ with $\gamma^{\prime}(0) \in \mathfrak{C}_{\gamma^{\prime}(0)}(P)$ and $\gamma(s)=x$. It implies the inclusion $\subseteq$. The other inclusion $(\supseteq)$ obtains just by putting $x=\gamma(s)$ and then considering the definition of the future cylinder.

By an analogue way one can prove item $(b)$.

Let us prove $(c)$. Consider $\rho_{+}: T u b_{r}^{+}(P) \longrightarrow \mathbb{R}, x \mapsto d_{F}(P, x)$. That is $\rho_{+}$as it is in 1.4 .15 and then restrict it on $T u b_{r}^{+}(P)$. Note that as $x \in T u b_{r}^{+}(P)$, there exists a unique minimizing geodesic of the unit speed

$$
\gamma:\left[-d_{F}(P, x), 0\right] \longrightarrow T u b_{r}^{+}(P)
$$

joining $P$ to $x$ such that $\gamma(0)=x$ and $L\left(\left.\gamma\right|_{\left[-d_{F}(P, x), 0\right]}\right)=d_{F}(P, x)$. In particular for every $t_{0}, t_{1} \in$ $\left[-d_{F}(P, x), 0\right]$,

$$
d_{F}\left(\gamma\left(t_{0}\right), \gamma\left(t_{1}\right)\right)=\int_{t_{0}}^{t_{1}} F\left(\gamma^{\prime}\right) d t
$$

So according to EQ. 1.13 for some small $t<0$ one has

$$
\begin{aligned}
\frac{\rho_{+}(\gamma(t))-\rho_{+}(\gamma(0))}{t} & \geq-\frac{d_{F}(\gamma(t), x)}{t} \\
& =-\frac{\int_{t}^{0} F\left(\gamma^{\prime}\right) d t}{t}=1
\end{aligned}
$$

and so

$$
d \rho_{+}\left(\gamma^{\prime}(0)\right)=\left.\frac{d}{d t} \rho_{+}(\gamma(t))\right|_{t=0} \geq 1
$$

which means $d \rho_{+}(x) \neq 0$ or in other words each $x \in T u b_{r}^{+}(P)$ is a regular point of $\rho_{+}$. So by Implicit Function Theorem

$$
\rho_{+}^{-1}(s)=C_{s}^{+}(P)
$$

where $\rho_{+}(x)=s$, is an embedded and closed submanifold of codimension 1. By an analogue argument $C_{s}^{-}(P)$ is an embedded and closed submanifold of codimension 1 .

To prove $(d)$, with respect to the definition of the future cylinder we have that

$$
P \| C_{t}^{+}(P) \text { and } d_{F}\left(P, C_{t}^{+}(P)\right)=t
$$

which means $\gamma$ minimizes the distance from $P$ to $C_{t}^{+}(P)$. Now by Lemma 1.3 .13 we have that $\gamma(t)$ is also orthogonal to the cylinder.

The proof of $(e)$ can be done similarly.

For the last two items, considering the point that $T_{x} C_{s}^{+}(P) \subset T_{x} M$ and item $(d)$ the proof of item $(f)$ is straightforwardly and the proof of $(g)$ is similarly. 
Remark 1.4.24. Observe that in Lemma 1.4.23, as an equivalent relation to item (a) one can write

$$
C_{s}^{+}(P)=\left\{\exp _{p}(s v) \mid p \in P \text { and } v \in \mathfrak{C}_{p}^{1}(P)\right\}
$$

Remark 1.4.25. According to the definitions of the cylinders and tubular neighborhoods and Lemma 1.4.23 one can have

$$
C_{r}^{+}(P)=\partial T u b_{r}^{+}(P) \text { and } C_{r}^{-}(P)=\partial T u b_{r}^{-}(P) .
$$

Moreover far from $P$ the (possibly degenerated) cylinders $\hat{C}_{s}^{+}(P)$ and $\hat{C}_{s}^{+}(P)$ might be ugly in the sense that the distance function $d_{F}$ may not be smooth or given a point $x$ far from $P$, there exist two separated minimizing geodesics joining $P$ to $x$.

Remark 1.4.26. Note that according to Lemma 1.4.23, another equivalent definition of the future (past) cylinder would be using the item (a) (item (b)) of this Lemma.

Lemma 1.4.27. Given submanifolds $C, D$ of a Finsler manifold $(M, F)$, the following three assertions are equivalent:

a) $C \| D$;

b) $C \subset C_{r}^{-}(D)$;

c) $D \subset C_{r}^{+}(C)$.

Proof. First let us prove the equivalence of assertions (b) and (c). Suppose that $D \subset C_{r}^{+}(C)$. Given $x \in C$, by the definition of the future cylinder,

$$
d_{F}(x, D)=r
$$

which by the definition of the past cylinder implies $x \in C_{r}^{-}(D)$. By the same argument one can prove the return.

Now let us show that $C \| D$ if and only if $C \subset C_{r}^{-}(D)$. If $C \| D$, by Definition 1.4.9, we have that

$$
d_{F}(x, D)=r,
$$

for every $x \in C$. From this equality and the definition of the past cylinder one gets that $x \in C_{r}^{-}(D)$. It means $C \subset C_{r}^{-}(D)$. Now in order to prove the converse, $C \subset C_{r}^{-}(D)$ points out that for every

$$
x \in C, d_{F}(x, D)=r .
$$

Comparing this equality with Definition 1.4 .9 gives implies $C \| D$.

Proposition 1.4.28. Let $(M, F)$ be a Finsler manifold and $P$ a precompact submanifold of it. Then

(a) Given a foliation by future cylinders $\left\{C_{s_{i}}^{+}(P)\right\}_{s_{i} \in\left(0, r^{+}\right)}$in $T u b_{r^{+}}^{+}(P)$, for $i<j$, we have that

$$
C_{s_{i}}^{+}(P) \| C_{s_{j}}^{+}(P)
$$


(b) Given a foliation by past cylinders $\left\{C_{s_{i}}^{-}(P)\right\}_{s_{i} \in\left(0, r^{-}\right)}$in $T u b_{r^{-}}^{-}(P)$, for $i<j$, we have that

$$
C_{s_{j}}^{+}(P) \| C_{s_{i}}^{+}(P)
$$

Proof. Because the proofs of both items follows the same strategies, we will be contented with only providing the proof of item $(a)$. For every $x \in C_{s_{j}}^{+}(P)$, there exists a unique unit speed minimizing geodesic $\gamma_{x}$ such that

$$
\gamma_{x}^{\prime}(0) \in \mathfrak{C}_{\gamma_{x}(0)}(P)
$$

and $\gamma_{x}\left(s_{j}\right)=x$. As we have a foliation by the future cylinders and $s_{i}<s_{j}$ then $\gamma_{x}\left(s_{i}\right) \in C_{s_{i}}^{+}(P)$. It means that the piece of $\gamma_{x}$ which joins $C_{s_{i}}^{+}(P)$ to $C_{s_{j}}^{+}(P)$ is of length $s_{j}-s_{i}$. Notice that $s_{i}$ and $s_{j}$ do not depend on $x$ and so

$$
C_{s_{i}}^{+}(P) \| C_{s_{j}}^{+}(P)
$$

with $d_{F}\left(C_{s_{i}}^{+}(P), C_{s_{j}}^{+}(P)\right)=s_{j}-s_{i}$.

\subsection{The past metric $\tilde{F}(v)=F(-v)$ and whose geometric objects}

Lemma 1.5.1. Let $(M, F)$ be a Finsler space and $\nabla^{V}$ the Chern connection, where $V$ is a non-zero vector field on an open $\mathcal{U} \subset M$. Define $\tilde{F}: T M \longrightarrow \mathbb{R}$ with

$$
\tilde{F}(y):=F(-y)
$$

Then $(M, \tilde{F})$ is a Finsler space whose fundamental tensor is given by

$$
\tilde{g}_{V}=g_{-V}
$$

Proof. First one needs to check the conditions 1 and 2 in Definition 1.2.13. Because of smoothness of $F$ on $T M \backslash 0$ and being positively homogeneous of degree 1, one can straightforwardly check those properties for $\tilde{F}$. Let us by using relation 1.1 find $\tilde{g}_{V}$.

$$
\begin{aligned}
\tilde{g}_{V}(X, Y) & =\left.\frac{1}{2} \frac{\partial^{2}}{\partial s \partial t}\left[\tilde{F}^{2}(V+s X+t Y)\right]\right|_{s=t=0}=\left.\frac{1}{2} \frac{\partial^{2}}{\partial s \partial t}\left[F^{2}(-V-s X-t Y)\right]\right|_{s=t=0} \\
& =g_{-V}(-X,-Y)=g_{-V}(X, Y)
\end{aligned}
$$

where $X, Y \in \mathfrak{X}(\mathcal{U})$. EQ. 1.14 means that $\tilde{g}_{V}=g_{-V}$ which also guarantees that $\tilde{g}_{V}$ is a positive definite symmetric bilinear form as $g_{V}$ is so. According to the definition of the Finsler metric, $\tilde{F}$ is a Finsler metric and so $(M, \tilde{F})$ is a Finsler space.

Lemma 1.5.2. Let $\tilde{C}_{V}$ be the Cartan tensor of the Finsler metric $\tilde{F}$. Then it is equal to

$$
\tilde{C}_{V}=-C_{-V}
$$

where $C_{V}$ is the Cartan tensor of F. 
Proof. According to EQ. 1.4 we have

$$
\begin{aligned}
\tilde{C}_{V}(X, Y, Z) & =\frac{1}{4} \frac{\partial^{3}}{\partial s_{3} \partial s_{2} \partial s_{1}}\left[\tilde{F}^{2}\left(V+s_{1} X+s_{2} Y+s_{3} Z\right)\right]_{s_{i}=0} \\
& =\frac{1}{4} \frac{\partial^{3}}{\partial s_{3} \partial s_{2} \partial s_{1}}\left[F^{2}\left(-V-s_{1} X-s_{2} Y-s_{3} Z\right)\right]_{s_{i}=0} \\
& =C_{-V}(-X,-Y,-Z)=-C_{-V}(X, Y, Z)
\end{aligned}
$$

for $X, Y, Z \in \mathfrak{X}(\mathcal{U})$.

Lemma 1.5.3. Let $\tilde{\nabla}^{V}$ and $\nabla^{V}$ be the Chern connections of $\tilde{F}$ and $F$, respectively. Then

$$
\tilde{\nabla}^{V}=\nabla^{-V}
$$

Proof. Because of the unicity of the Chern connection that is enough to show that $\tilde{\nabla}^{V}$ and $\nabla^{-V}$ both solve the same equation. That is, according to Definition 1.3.4, that is enough to prove that $\nabla^{-V}$ is torsion-free and almost $\tilde{g}$-compatible. As $\nabla^{V}$ is torsion-free, in particular for $-V$, we have

$$
\nabla_{X}^{-V} Y-\nabla_{Y}^{-V} X=[X, Y]
$$

which provides the torsion-freeness of $\nabla^{-V}$. Let us show that $\nabla^{-V}$ is almost $\tilde{g}$-compatible.

$$
\begin{aligned}
X\left(\tilde{g}_{V}(Y, Z)\right)=X\left(g_{-V}(Y, Z)\right) & =g_{-V}\left(\nabla_{X}^{-V} Y, Z\right)+g_{-V}\left(Y, \nabla_{X}^{-V} Z\right)+2 C_{-V}\left(\nabla_{X}^{-V}(-V), Y, Z\right) \\
& =\tilde{g}_{V}\left(\nabla_{X}^{-V} Y, Z\right)+\tilde{g}_{V}\left(Y, \nabla_{X}^{-V} Z\right)+2 \tilde{C}_{V}\left(\nabla_{X}^{-V} V, Y, Z\right) .
\end{aligned}
$$

So $\nabla^{-V}$ is the Chern connection of $\tilde{F}$ which means $\nabla^{-V}=\tilde{\nabla}^{V}$

Lemma 1.5.4. Consider two piecewise smooth curves $t \longrightarrow \gamma(t)$ and $t \longrightarrow \tilde{\gamma}(t):=\gamma(-t)$ in $M$. Then $\gamma$ is a geodesic with respect to the Finsler metric $F$ if and only if $\tilde{\gamma}$ is a geodesic with respect to the Finsler metric $\tilde{F}$. In particular they have the same velocity.

Proof. First of all notice that for $\gamma:[0, a] \longrightarrow M$ we have $\tilde{\gamma}:[-a, 0] \longrightarrow M$. As $\tilde{\gamma}(t)=\gamma(-t)$ we have $\tilde{\gamma}^{\prime}(t)=-\gamma^{\prime}(-t)$. Now according to Lemma 1.5.3, we have

$$
\tilde{\nabla}_{\tilde{\gamma}^{\prime}}^{\tilde{\gamma}^{\prime}} \tilde{\gamma}^{\prime}=\nabla_{\tilde{\gamma}^{\prime}}^{-\tilde{\gamma}^{\prime}} \tilde{\gamma}^{\prime}=\nabla_{\gamma^{\prime}}^{\gamma^{\prime}} \gamma^{\prime}
$$

In particular we have $\gamma$ is a geodesic with respect to $F$ if and only if $\tilde{\gamma}$ is a geodesic with respect to $\tilde{F}$. For the last assertion, note that

$$
\tilde{F}\left(\tilde{\gamma}^{\prime}\right)=F\left(-\tilde{\gamma}^{\prime}\right)=F\left(\gamma^{\prime}\right)
$$

Lemma 1.5.5. Given a submanifold $L \subset M$, let $\mathfrak{C}_{p}(L)$ and $\tilde{\mathfrak{C}}_{p}(L)$ be the cones, resp., with respect to $F$ and $\tilde{F}$. Then, given $v \in T_{p} M, v \in \mathfrak{C}_{p}(L)$ if and only if $-v \in \tilde{\mathfrak{C}}_{p}(L)$. 
Proof. Using the Definition 1.3.1 and Lemma 1.5.1, for every $u \in T_{p} L$ and given some $v \in T_{p} M$, one can write $v \in \mathfrak{C}_{p}(L)$ if and only if $g_{v}(v, u)=0$ if and only if $\tilde{g}_{-v}(-v, u)=0$ if and only if $-v \in \tilde{\mathfrak{C}}_{p}(L)$.

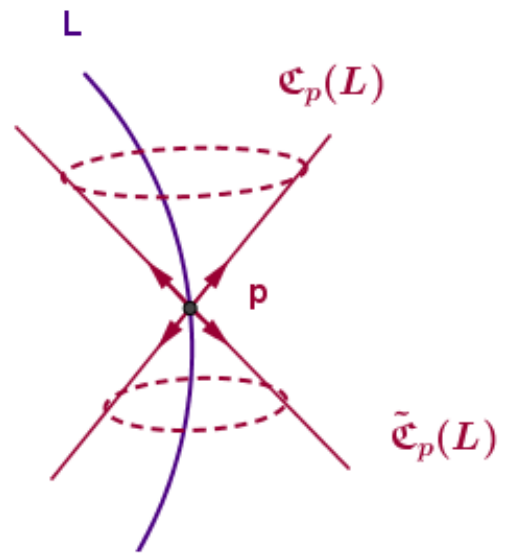

Figure 1.11: the cones $\mathfrak{C}_{p}(L)$ and $\tilde{\mathfrak{C}}_{p}(L)$

Lemma 1.5.6. Given a submanifold $L \subset M$, let $C_{r}^{ \pm}(L)$ and $\tilde{C}_{r}^{ \pm}(L)$ be, resp., the future and past cylinders of radius $r$ and axis $L$ with respect to $F$ and the future and past cylinders of radius $r$ with respect to of $\tilde{F}$ and axis $L$. Then

$$
C_{r}^{+}(L)=\tilde{C}_{r}^{-}(L) \text { and } C_{r}^{-}(L)=\tilde{C}_{r}^{+}(L)
$$

Proof. Let $p \in M$ and $\gamma:[0, r] \longrightarrow M$ be a piecewise smooth curve with $\gamma(0) \in L$ and $\gamma(r)=p$. Consider $\tilde{\gamma}(t):=\gamma(t)$. Using the items $(a)$ and $(b)$ of Lemma 1.4.23, Lemma 1.5.4, and Lemma 1.5.5, we have the following equivalences. $p=\gamma(r) \in C_{r}^{+}(L)$ if and only if $\gamma$ is the unique geodesic with $\gamma^{\prime}(0) \in \mathfrak{C}_{\gamma(0)}^{1}(L)$ if and only if $\tilde{\gamma}$ is the unique geodesic with $\tilde{\gamma}^{\prime}(0) \in \mathfrak{C}_{\tilde{\gamma}(0)}^{1}(L)$ if and only if $p=\tilde{\gamma}(-r) \in C_{r}^{-}(L)$. It provides the equality

$$
C_{r}^{+}(L)=\tilde{C}_{r}^{-}(L)
$$

and by the same argument, mutatis mutandis, one can prove the equality

$$
C_{r}^{-}(L)=\tilde{C}_{r}^{+}(L)
$$

Lemma 1.5.7. Let $\exp _{x}$ and $\exp _{p}$ be the exponential maps related to the metrics $\tilde{F}$ and $F$, respectively. Then

$$
\exp _{x}(t(-v))=\exp _{x}(-t v)
$$

Proof. According to Lemma 1.5.4, assume that $\tilde{\gamma}_{-v}(t)$ and $\gamma_{v}(t)$ are the geodesics such that $\tilde{\gamma}_{-v}(0)=$ $\gamma_{v}(0)=x$ and $\tilde{\gamma}_{-v}^{\prime}(0)=-v=-\gamma_{v}^{\prime}(0)$. Then by the Definition of the exponential map 1.3.15, we have

$$
\exp _{x}(t(-v))=\tilde{\gamma}_{-v}(t)=\gamma_{v}(-t)=\exp _{x}(-t v)
$$


Observe that here $t<0$ and so $-t>0$.

Lemma 1.5.8. Let us recall Lemma 1.3.21. Given a geodesic $\gamma:[0, l] \longrightarrow M$ and a variation $f:(-\epsilon, \epsilon) \times[0, l] \longrightarrow M$ along $\gamma$ with whose field of variation $J(t)$, let $\tilde{f}(s, t):=f(s,-t)$. Then

$$
\tilde{J}(t)=J(-t),
$$

where $\tilde{J}$ is the variational field associated to $\tilde{f}$. In particular $r$ is a J-conjugate instant if and only if $-r$ is a $\tilde{J}$-conjugate instant.

Proof. Observe that $\tilde{f}:(-\epsilon, \epsilon) \times[-l, 0] \longrightarrow M$ is a variation of the geodesic $\tilde{\gamma}:[-l, 0] \longrightarrow M$. Then

$$
\tilde{J}(t)=\frac{\partial \tilde{f}}{\partial s}(0, t)=\frac{\partial f}{\partial s}(0,-t)=J(-t) .
$$

The last assertion is a direct result of this equation.

Lemma 1.5.9. Given a submanifold $L$ of a Finsler manifold $(M, F)$, assume that $\gamma:[0, l] \longrightarrow M$ is a geodesic such that $\gamma(0) \in L$ and $\gamma^{\prime}$ is $g_{\gamma^{\prime}}$-orthogonal to $L$ and $J$ is a $L$-Jacobi field along $\gamma$. Then for the geodesic $\tilde{\gamma}(t)=\gamma(-t), \tilde{J}(t)$ is a L-Jacobi field along $\tilde{\gamma}$. Moreover, $r$ is a L-focal instant for $F$ if and only if $-r$ is a $L$-focal instant for $\tilde{F}$

Proof. Let us recall Definition 1.3.20. Then according to Lemma 1.5.8, properties of the tan projection, and the hypothesis,

$$
\begin{aligned}
\tan _{\tilde{\gamma}^{\prime}} \tilde{J}^{\prime}(0) & =\tan _{\gamma^{\prime}}\left(-J^{\prime}(0)\right)=-\tan _{\gamma^{\prime}} J^{\prime}(0)=-S_{\gamma^{\prime}}(J(0)) \\
& =-\tan _{\gamma^{\prime}} \nabla_{J(0)}^{\gamma^{\prime}} \gamma^{\prime}=-\tan _{\tilde{\gamma}^{\prime}} \tilde{\nabla}_{J(0)}^{\tilde{\gamma}^{\prime}}\left(-{\tilde{\gamma^{\prime}}}^{\prime}\right)=\tan _{\tilde{\gamma}^{\prime}} \tilde{\nabla}_{J(0)}^{\tilde{\gamma}^{\prime}} \tilde{\gamma}^{\prime} \\
& =\tilde{S}_{\tilde{\gamma}^{\prime}}(J(0))=\tilde{S}_{\tilde{\gamma}^{\prime}}(\tilde{J}(0)) .
\end{aligned}
$$

In calculations we also used EQ. 1.7 two times. As a consequence one has that $\tilde{J}$ is a $L$-Jacobi field. The last assertion is a direct result from the equality $J(r)=\tilde{J}(-r)$. 


\section{Chapter 2}

\section{Randers spaces}

\subsection{Introduction}

As Randers spaces provide us some nice examples that give one better understanding of this work we devote a chapter to this type of Finsler metrics. Let us recall Definition 1.2.16. From Finslerian geometry's point of view, Randers metric is a metric with a simple form which is obtained by a Riemannian metric $\alpha$ together with a 1 -form $\beta$ (provided that whose dual norm is less than 1, i.e. $\alpha(\beta)<1)$. Because of this the study of this metric, in particular in some special cases, is easier than the other classes of Finsler metrics. Furthermore, this class of Finsler metric has a variety of applications in other sciences, like magnetic field [GW11], wildfire [Mar16], Zermelo's navigation problem [CG12], seismological studies [ABS03], etc. [Got09, CJS14, BRS ${ }^{+}$04, Voi11, PW12, Kri10, Jav12, BM07]. Indeed Shen in [She01a] showed that given a Riemannian space $(M, h)$ and a vector field on it such that $\|W\|<1$, then there exists a type of Finsler metric which is called Randers metric whose norm is given by Proposition 2.2.2. Moreover in $\left[\mathrm{BRS}^{+} 04\right]$ it is shown that every Randers metric arises as the solution to a Zermelo's problem of navigation associated to the data $(M, h, W)$.

\subsection{Zermelo navigation and Randers metric}

Zermelo aims to find the paths of shortest travel time in a Riemannian manifold $(M, h)$, under the influence of a time independent wind which is represented by a vector field $W\left[\mathrm{BRS}^{+} 04\right]$. To get some intuition about this matter, a brief explanation is given below. Afterward it is explained how to find a Randers metric associated to a navigation problem.

Let $(M, h)$ be a Riemannian space. For every $y \in T_{x} M$ think of $\|y\|$ as the taken time to travel from $0_{x}$ to the tip of $y$. Now assume that we have a mild wind $W$ on $M$, namely $h(W, W)<1$ or in other words its velocity is less than 1 . Before having this wind the locus of all unitary vectors with the foot point $0_{x}$ at $t=1$ was the geometric sphere. That is, the journey with the beginning point $x$ along any unitary direction $u$ takes 1 second. Note that the presence of the wind affects the taken time to travel from the foot point of a unitary vector and also the locus. So one needs to manipulate the metric and as the first consequence the Riemannian geodesics are not the shortest paths in this new ambient any more. In this new ambient the taken time to travel from a point $x$ depends on the direction and in the general case depends on the both components of the initial point and the direction of the travel. So the Riemannian metric $h$ does not give the travel time and 
so we need a new metric $F$ which considers the foot point and the direction of the travel. It means $F$ is a function that lives in the tangent bundle $T M$. With this new metric the locus of the unitary vectors from the foot point $x \in M$ which is called an "indicatrix" and was mentioned in Chapter 1,

$$
\Sigma^{F}=\left\{u \in T_{x} M: F(u)=1\right\},
$$

does not coincide with the unitary geometric sphere associated to the metric $h$, unless for some special winds $W$. See Picture 2.1 in which some of the geometric spheres before the wind $W=\frac{1}{2}$ and after it are shown.

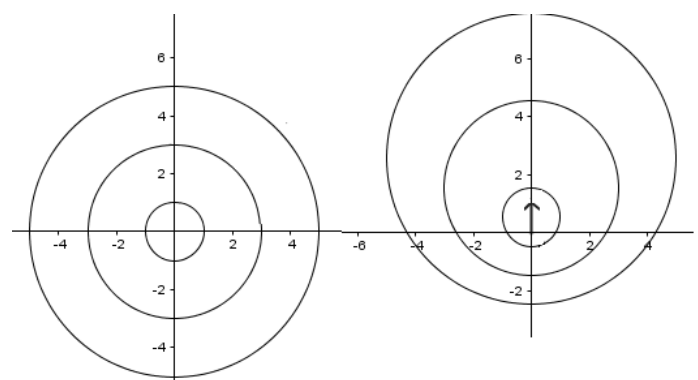

Figure 2.1: A sample of geometric spheres

Convention. By the Zermelo's data $(M, h ; W)$, we mean a Riemannian space $(M, h)$ with a wind $W$ on $M$ such that $\|W\|<1$. Also we show the associated Randers metric with $F$.

\subsubsection{The explicit expression of $F$ and some geometric objects}

Given the Zermelo's data $(M, h ; W)$, we will find the associated Randers metric $F$.

Proposition 2.2.1. [BRS $\left.S^{+} 04\right]$ A strongly convex Finsler metric $F$ is of Randers type if and only if it solves the Zermelo's navigation problem on a Riemannian manifold $(M, h)$, under the influence of a wind $W$ which satisfies $h(W, W)<1$. Also, $F$ is Riemannian if and only if $W=0$.

Now according to this proposition let's calculate the Randers metric $F$ associated to the Zermelo's data $(M, h ; W)$.

Proposition 2.2.2. Consider the Zermelo's data $(M, h ; W)$. Then its associated Randers metric is given by

$$
F(y)=\left[\frac{\sqrt{h^{2}(W, y)+\lambda h(y, y)}}{\lambda}-\frac{h(W, y)}{\lambda}\right]
$$

in which $\lambda=1-h(W, W)$.

Proof. Let's start with a unitary vector $u$, i.e. $h(u, u)=1$. When the wind affects on it we get the consequent vector $v=u+W$ and so $u=v-W$. Now the plan is to find the new metric $F$ for which $F(v)=1$.

$$
\begin{aligned}
h(u, u)=h(v-W, v-W) & =h(v, v)+h(W, W)-2 h(v, W) \\
& =\|v\|^{2}+\|W\|^{2}-2\|v\| .\|W\| \cos \theta=1 .
\end{aligned}
$$

Putting $\lambda=1-\|W\|^{2}$, one gets

$$
\|v\|^{2}-(2|| W|| \cos \theta)\|v\|-\lambda=0 .
$$


Now as $\|W\|<1$ we have $\|v\| \neq 0$ and so

$$
\|v\|=\|W\| \cos \theta+\sqrt{\|W\|^{2} \cos ^{2} \theta+\lambda}
$$

Denoting $p=\|W\| \cos \theta$ and $q=\sqrt{\|W\|^{2} \cos ^{2} \theta+\lambda}$, eventually the following expression of $F$ for the unitary vectors is obtained

$$
F(v)=1=\frac{\|v\|}{p+q}=\|v\| \cdot \frac{q-p}{q^{2}-p^{2}}=\frac{\sqrt{h^{2}(v, W)+\lambda h(v, v)}}{\lambda}-\frac{h(v, W)}{\lambda} .
$$

Before proceeding with the expression of the metric for each arbitrary non-zero vector, we shall mention a useful lemma that we take benefit of.

Claim 2.2.3. Given $x \in M$, suppose that

$$
\Sigma_{r}^{F}=\left\{v \in T_{x} M: F(v)=r\right\} \text { and } \Sigma_{1}^{F}=\left\{v \in T_{x} M: F(v)=1\right\} .
$$

Then

$$
\Sigma_{r}^{F}=r \Sigma_{1}^{F}
$$

Proof. Observe that if $y \in r \Sigma_{1}^{F}=\left\{r v \in T_{x} M: r F(v)=r\right\}$, then $y=r v \in \Sigma_{r}^{F}$. Moreover for each $y \in \Sigma_{r}^{F}$ one can write $y=r v$ where $r=F(y)$ and $v=\frac{y}{F(y)}$. Then $y=r v \in r \Sigma_{1}^{F}$.

According to Claim 2.2.3, $v \in \Sigma_{1}^{F}$ if and only if $r v \in \Sigma_{r}^{F}$, for every $r>0$. Also for every non-zero vector $y$ belonging to the tangent bundle we have

$$
F(y)=F(r v)=r F(v)
$$

where $r=F(y)$ and $v=\frac{y}{F(y)}$. Eventually using above relations and explanations, the expression of the metric $F$ is given as follows:

$$
\begin{aligned}
& F(y)=F(y) \cdot F\left(\frac{y}{F(y)}\right)=F(y) \cdot\left[\frac{\sqrt{h^{2}\left(W, \frac{y}{F(y)}\right)+\lambda h\left(\frac{y}{F(y)}, \frac{y}{F(y)}\right)}}{\lambda}-\frac{h\left(W, \frac{y}{F(y)}\right)}{\lambda}\right] \\
& F(y)=\left[\frac{\sqrt{h^{2}(W, y)+\lambda h(y, y)}}{\lambda}-\frac{h(W, y)}{\lambda}\right]
\end{aligned}
$$

Lemma 2.2.4. Given $x \in M$, suppose that

$$
\Sigma_{r}^{F}=\left\{v \in T_{x} M: F(v)=r\right\} \text { and } \Sigma_{r}^{h}=\left\{v \in T_{x} M:\|v\|=r\right\}
$$

are the indicatrices of radii $r$ related to the metrics $F$ and $h$, respectively. Then

$$
\Sigma_{r}^{h}+r W(x)=\Sigma_{r}^{F}
$$

Proof. According to calculations in Proposition 2.2.2, $F(v)=1$ if and only if $h(v-W, v-W)=1$ which means $\Sigma_{1}^{F}$ is obtained by a translation of $\Sigma_{1}^{h}$ along $W(x)$. Namely, we have $\Sigma_{1}^{F}=\Sigma_{1}^{h}+W(x)$ 
and so

$$
r \Sigma_{1}^{F}=r \Sigma_{1}^{h}+r W(x)
$$

Now by Claim 2.2.3 and the of the geometric sphere we get $r \Sigma_{1}^{F}=\Sigma_{r}^{F}$ and $r \Sigma_{1}^{h}=\Sigma_{r}^{h}$ with which the desired equation is obtained.

As a direct consequence of Lemma 2.2.4 we have the following corollary.

Corollary 2.2.5. For an arbitrary vector $y \in T M \backslash\{0\}$, we have

$$
\left\|\frac{y}{F(y)}-W\right\|=1 \text { and } F\left(\frac{y}{\|y\|}+W\right)=1 .
$$

Lemma 2.2.6. Consider the Zermelo's data $(M, h ; W)$ with the associated Randers metric $F$. Then,

1. the fundamental tensor is given as

$$
g_{y}(v, u)=\left(\frac{a(u, y)}{\alpha(y)}+\beta(u)\right)\left(\frac{a(v, y)}{\alpha(y)}+\beta(v)\right)+\frac{F(y)}{\alpha(y)}\left[a(v, u)-\frac{a(v, y)}{\alpha(y)} \frac{a(u, y)}{\alpha(y)}\right]
$$

2. In particular

$$
g_{y}(y, u)=F(y)\left[\frac{a(u, y)}{\alpha(y)}+\beta(u)\right]=\frac{F(y)}{\lambda \alpha(y)} h(u, y-F(y) W) .
$$

Here $\lambda=1-h(W, W)$

Proof. According to Proposition 2.2.2,

$$
F(y)=\sqrt{\frac{h^{2}(y, W)+\lambda h(y, y)}{\lambda^{2}}}-\frac{h(y, W)}{\lambda} .
$$

Put $F(y)=\alpha(y)+\beta(y)$ with $\alpha(y)=\sqrt{a(y, y)}$ and let us calculate $a(u, y)$ and $\beta(y)$. Using the polarization identity for the real vector spaces and considering $a(y, y)=\frac{h^{2}(y, W)+\lambda h(y, y)}{\lambda^{2}}$ one gets

$$
a(u, y)=\frac{1}{\lambda^{2}}[h(u, W) h(y, W)+\lambda h(u, y)] \text { and } \beta(y)=h\left(y,-\frac{W}{\lambda}\right) .
$$

Now, let's calculate the fundamental tensor. According to EQ. 1.1

$$
\begin{aligned}
g_{y}(v, u) & =\left.\frac{1}{2} \frac{\partial^{2}}{\partial s \partial t}\left[F^{2}(y+t v+s u)\right]\right|_{s=t=0}=\left.\left.\frac{1}{2} \frac{\partial}{\partial s}\right|_{s=0} \frac{\partial}{\partial t}\right|_{t=0}\left[F^{2}(y+t v+s u)\right] \\
& =\left.\frac{1}{2} \frac{\partial}{\partial s}\right|_{s=0}\left[\left.2 F(y+s u) \frac{\partial}{\partial t}\right|_{t=0} F(y+s u+t v)\right] \\
& =\left.\frac{\partial}{\partial s}\right|_{s=0}\left[(\alpha(y+s u)+\beta(y+s u)) \times\left.\frac{\partial}{\partial t}\right|_{t=0}(\alpha(y+s u+t v)+\beta(y+s u+t v))\right] .
\end{aligned}
$$

First let's calculate

$$
\left.\frac{\partial}{\partial t}\right|_{t=0} \alpha(y+s u+t v)=\left.\frac{\partial}{\partial t}\right|_{t=0} \sqrt{a(y+s u+t v, y+s u+t v)}=\frac{a(v, y+s u)}{\sqrt{a(y+s u, y+s u)}} .
$$

Now,

$$
\begin{aligned}
\left.\frac{\partial}{\partial s}\right|_{s=0} \frac{a(v, y+s u)}{\sqrt{a(y+s u, y+s u)}} & =\frac{1}{\alpha^{2}(y)}\left[a(v, u) \alpha(y)-a(v, y) \cdot \frac{a(y, u)}{\alpha(y)}\right] \\
& =\frac{1}{\alpha(y)}\left[a(v, u)-\frac{a(v, y)}{\alpha(y)} \frac{a(u, y)}{\alpha(y)}\right] .
\end{aligned}
$$


As 2.4, one has

$$
\left.\frac{\partial}{\partial s}\right|_{s=0} \alpha(y+s u)=\frac{a(u, y)}{\alpha(y)} .
$$

Replacing above calculations in 2.3 we get

$$
\begin{aligned}
g_{y}(v, u) & =\left(\frac{a(u, y)}{\alpha(y)}+\beta(u)\right)\left(\frac{a(v, y)}{\alpha(y)}+\beta(v)\right)+\frac{(\alpha(y)+\beta(y))}{\alpha(y)}\left[a(v, u)-\frac{a(v, y)}{\alpha(y)} \frac{a(u, y)}{\alpha(y)}\right] \\
& =\left(\frac{a(u, y)}{\alpha(y)}+\beta(u)\right)\left(\frac{a(v, y)}{\alpha(y)}+\beta(v)\right)+\frac{F(y)}{\alpha(y)}\left[a(v, u)-\frac{a(v, y)}{\alpha(y)} \frac{a(u, y)}{\alpha(y)}\right] .
\end{aligned}
$$

as well as

$$
g_{y}(y, u)=F(y)\left[\frac{a(u, y)}{\alpha(y)}+\beta(u)\right]
$$

which are two of the desired relations in the expression of Lemma. Now replacing $a(u, y)$ and $\beta(u)$ obtained from the polarization identity at this equality we finally get

$$
\begin{aligned}
g_{y}(y, u) & =F(y)\left[\frac{h(u, W) h(y, W)+\lambda h(u, y)}{\lambda^{2} \alpha(y)}-h\left(\frac{W}{\lambda}, u\right)\right] \\
& =\frac{F(y)}{\lambda \alpha(y)}\left[h\left(u, \frac{W}{\lambda}\right) h(y, W)+h(u, y)-h(\alpha(y) W, u)\right] \\
& =\frac{F(y)}{\lambda \alpha(y)}\left[h\left(u, \frac{W}{\lambda} h(y, W)+y-\alpha(y) W\right)\right] \\
& =\frac{F(y)}{\lambda \alpha(y)}\left[h\left(u,\left(\frac{1}{\lambda} h(y, W)-\alpha(y)\right) W+y\right)\right] \\
& =\frac{F(y)}{\lambda \alpha(y)}[h(u, y-F(y) W)] .
\end{aligned}
$$

As we desired

Here we state a useful corollary which, for a nonzero vector $y$, helps us to find the cone $\mathfrak{C}_{y}$, using the Riemannian metric $h$. So we have the following corollary which is a direct consequence of Lemma 2.2.6.

Corollary 2.2.7. With the Zermelo's data $(M, h ; W)$ we have that for every non-zero vectors $u, y \in T_{p} M$,

$$
g_{y}(y, u)=0 \text { if and only if } h\left(u, \frac{y}{F(y)}-W\right)=0,
$$

or in other words, $y$ is in the cone if and only if $\frac{y}{F(y)}-W$ is in the horizontal space with respect to $h$.

\subsubsection{Some results on Randers transnormality}

Observe that in fact the gradient of a function in the Finsler case cannot be calculated as much as easy the Riemannian case. Even in the Randers-Minkowski case it demands some efforts to be calculated. So one needs to use some other techniques to show that a function is $F$-transnormal. In this part we provide some methods that help us to calculate the norm of the gradient in some special cases.

Lemma 2.2.8. Consider the Zermelo's data $(M, h ; W)$ with the associated Randers metric $F$. Let $\mathcal{U}$ be an open subset of Randers manifold $M$ and $f$ a smooth function on $\mathcal{U}$ with $d f \neq 0$. Then

$$
\nabla f-F(\nabla f) W=\lambda_{1} \bar{\nabla} f
$$

where $\lambda_{1}: M \longrightarrow \mathbb{R}>0$ with $\lambda_{1}=\frac{h(W, \nabla f)+\lambda F(\nabla f)}{F(\nabla f)}$ and $\nabla f$ and $\bar{\nabla} f$ are the gradients of $f$ with respect to $F$ and h, respectively. 
Proof. Consider the regular leaf $f^{-1}(c)$, that is $c$ is a regular value. We know that each such a leaf is an embedded submanifold of codimension 1. Note that according to Corollary 2.2.7, for $p \in f^{-1}(c)$ we have $v \in \mathfrak{C}_{p}\left(f^{-1}(c)\right)$ if and only if $v-F(v) W_{p} \in \mathcal{H}_{p}$. Here we are considering the decomposition

$$
\mathcal{H}_{p} \oplus \nu_{p}=T_{p} M
$$

with respect to $h$ where $\mathcal{H}_{p}=\left(T_{p} f^{-1}(c)\right)^{\perp}$ and $\nu_{p}=T_{p} f^{-1}(c)$. According to this relation and Lemma 1.3.2, $\mathcal{H}_{p}$ and $\mathfrak{C}_{p}\left(f^{-1}(c)\right)$ are each of dimensions 1 . Moreover $\bar{\nabla} f(p) \in \mathcal{H}_{p}$ and by Lemma 1.3 .32

$$
\nabla f(p) \in \mathfrak{C}_{p}\left(f^{-1}(c)\right)
$$

Hence from Corollary 2.2.7 the following equality can be posed as the relations between $\nabla f$ and $\bar{\nabla} f$.

$$
\nabla f-F(\nabla f) W=\lambda_{1} \bar{\nabla} f
$$

where $\lambda_{1}: M \longrightarrow \mathbb{R}$. Now let us prove that $\lambda_{1}>0$. First, from $\nabla f-F(\nabla f) W=\lambda_{1} \bar{\nabla} f$ we have

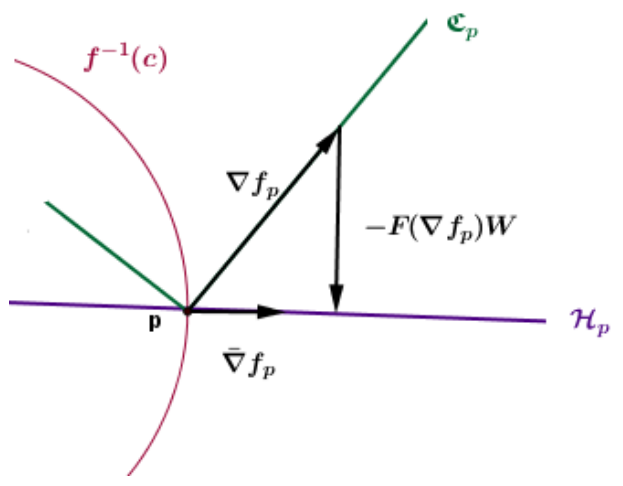

Figure 2.2: $c \in f(M)$

$$
g_{\nabla f}(\nabla f, \nabla f)-F(\nabla f) g_{\nabla f}(\nabla f, W)=\lambda_{1} g_{\nabla f}(\nabla f, \bar{\nabla} f) .
$$

By Cauchy-Schwarz inequality 1.2.10 we have $-g_{\nabla f}(\nabla f, W) \geq-F(\nabla f) F(W)$. Applying this inequality in EQ. 2.6 yields that

$$
\begin{aligned}
F^{2}(\nabla f)(1-F(W)) & =F^{2}(\nabla f)-F(W) F^{2}(\nabla f) \leq \lambda_{1} g_{\nabla f}(\nabla f, \bar{\nabla} f)=\lambda_{1} d f(\bar{\nabla} f) \\
& =\lambda_{1} h(\bar{\nabla} f, \bar{\nabla} f)=\lambda_{1}\|\bar{\nabla} f\|^{2} .
\end{aligned}
$$

In above calculations we also used the definition of gradient 1.3.28 twice. Let's see what is $1-F(W)$. Using relation 2.2.2,

$F(W)=\frac{1}{1-\|W\|^{2}}\left[\sqrt{h^{2}(W, W)+(1-\|W\|)}-h(W, W)\right]=\frac{1}{1-\|W\|^{2}}\left[\|W\|-\|W\|^{2}\right]=\frac{\|W\|}{1+\|W\|}$

which implies that

$$
1-F(W)=\frac{1+\|W\|-\|W\|}{1+\|W\|}=\frac{1}{1+\|W\|}>0 .
$$

So observe that from the relation $F^{2}(\nabla f)(1-F(W)) \leq \lambda_{1}\|\bar{\nabla} f\|^{2}$ in the case of non-singularity (which is also our case) we have $\lambda_{1}>0$. Let's find $\lambda_{1}$. According to Lemma 2.2.6 and definition of 
gradient for every $v$, we have

$$
\begin{aligned}
h(\bar{\nabla} f, v)=d f(v)=g_{\nabla f}(\nabla f, v) & =\frac{F(\nabla f)}{\lambda \alpha(\nabla f)}(h(\nabla f-F(\nabla f) W, v)) \\
& =h\left(\frac{F(\nabla f)}{\lambda \alpha(\nabla f)}(\nabla f-F(\nabla f) W), v\right)
\end{aligned}
$$

which implies that

$$
\bar{\nabla} f=\frac{F(\nabla f)}{\lambda \alpha(\nabla f)}(\nabla f-F(\nabla f) W)=\frac{F(\nabla f)}{\lambda F(\nabla f)+h(W, \nabla f)}(\nabla f-F(\nabla f) W) .
$$

Comparing this equality with relation 2.5 we get what we were looking for. Here in the last equality we used the fact that $F()=.\alpha()-.h\left(\frac{W}{\lambda},.\right)$.

Corollary 2.2.9. Consider the Zermelo's data $(M, h ; W)$ with the associated Randers metric F. Let $\mathcal{U}$ be an open subset of Finsler manifold $M$ and $f$ be a smooth function on $\mathcal{U}$ with $d f \neq 0$. Then

$$
\frac{\nabla f}{F(\nabla f)}-W=\frac{\bar{\nabla} f}{\|\bar{\nabla} f\|}
$$

Proof. Consider $\lambda_{1}$ defined in relation 2.5. Then according to Lemma 2.2.8,

$$
\frac{\nabla f}{F(\nabla f)}-W=\frac{\lambda_{1}}{F(\nabla f)} \bar{\nabla} f
$$

By Corollary 2.2.5 we have $\frac{\nabla f}{F(\nabla f)}-W \in \Sigma^{h}$ which means $\frac{\lambda_{1}}{F(\nabla f)}\|\bar{\nabla} f\|=1$ and so $\lambda_{1}=\frac{F(\nabla f)}{\|\nabla f\|}$.

Lemma 2.2.10. Consider the Zermelo's data $(M, h ; W)$ with the associated Randers metric $F$. Let $\mathcal{U}$ be an open subset of Finsler manifold $M$ and $f$ be a smooth function on $\mathcal{U}$ with $d f \neq 0$. Then $W$ is tangent to the (regular) leaves if and only if

$$
F(\nabla f)=\|\bar{\nabla} f\|
$$

in which $\|\|=.\sqrt{h(., .)}$. In particular

$$
\nabla f-F(\nabla f) W=\bar{\nabla} f
$$

Proof. We first recall Lemma 2.2.8. Also as $W$ is tangent to the leaves, $h(\bar{\nabla} f, W)=0=d f(W)=$ $g_{\nabla f}(\nabla f, W)$. So,

$$
\begin{aligned}
F^{2}(\nabla f) & =g_{\nabla f}(\nabla f, \nabla f)=g_{\nabla f}\left(\nabla f, F(\nabla f) W+\lambda_{1} \bar{\nabla} f\right) \\
& =F(\nabla f) g_{\nabla f}(\nabla f, W)+\lambda_{1} g_{\nabla f}(\nabla f, \bar{\nabla} f) \\
& =\lambda_{1} g_{\nabla f}(\nabla f, \bar{\nabla} f)=\lambda_{1} d f(\bar{\nabla} f)=\lambda_{1} h(\bar{\nabla} f, \bar{\nabla} f) \\
& =\lambda_{1}\|\bar{\nabla} f\|^{2} .
\end{aligned}
$$

Note that according to Corollary 2.2.9, $\lambda_{1}=\frac{F(\nabla f)}{\|\nabla f\|}$. Replacing $\lambda_{1}$ in this equality gives

$$
F^{2}(\nabla f)=\frac{F(\nabla f)}{\|\bar{\nabla} f\|}\|\bar{\nabla} f\|^{2}
$$


which means

$$
F(\nabla f)=\|\bar{\nabla} f\|
$$

and so $\lambda_{1}=1$.

Now assume that $F(\nabla f)=\|\bar{\nabla} f\|$. By Corollary 2.2.9, $\lambda_{1}=1$ and so by Lemma, 2.2.8 $\nabla f=$ $F(\nabla f) W+\bar{\nabla} f$. Therefore

$$
\begin{aligned}
F^{2}(\nabla f)= & g_{\nabla f}(\nabla f, \nabla f)=F(\nabla f) g_{\nabla f}(\nabla f, W)+g_{\nabla f}(\nabla f, \bar{\nabla} f) \\
& =F(\nabla f) g_{\nabla f}(\nabla f, W)+d f(\bar{\nabla} f) \\
& =F(\nabla f) g_{\nabla f}(\nabla f, W)+h(\bar{\nabla} f, \bar{\nabla} f) .
\end{aligned}
$$

Applying the hypothesis in the last equality gives that

$$
F^{2}(\nabla f)=F(\nabla f) g_{\nabla f}(\nabla f, W)+F^{2}(\nabla f)
$$

Since we are in the regular part $F(\nabla f) \neq 0$ and thus $g_{\nabla f}(\nabla f, W)=0$. Accordingly $W$ is tangent to the leaves. See Picture 2.3 and compare it with picture 2.2 .

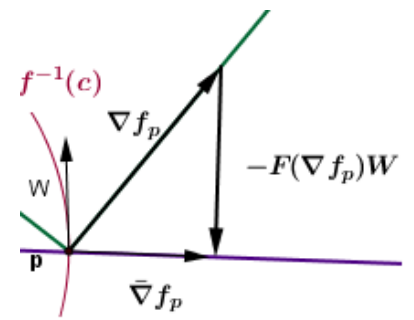

Figure 2.3: $W_{p} \in T_{p} f^{-1}(c)$

Corollary 2.2.11. Consider the Zermelo's data $(M, h ; W)$ with the associated Randers metric $F$. Let $f: M \longrightarrow \mathbb{R}$ be a smooth function and $W$ tangent to the leaves. Then $f$ is h-transnormal with $\|\bar{\nabla} f\|^{2}=\mathfrak{b}(f)$ if and only if it is F-transnormal with $F^{2}(\nabla f)=\mathfrak{b}(f)$ such that $\mathfrak{b} \in C^{2}(f(M))$.

Proof. According to Lemma 2.2.10, as $W$ is tangent to the leaves,

$$
F^{2}(\nabla f)=\|\bar{\nabla} f\|^{2}
$$

Now considering this equation and Definitions 1.3.33 and 1.3.34 the assertion is clear.

Lemma 2.2.12. Given the Zermelo's data $(M, h ; W)$ with the associated Randers metric $F$, let $f: M \longrightarrow \mathbb{R}$ be a smooth function and

$$
h(W, \bar{\nabla} f)=\tilde{\mathfrak{b}} \circ f
$$

for some $\tilde{\mathfrak{b}}: M \longrightarrow \mathbb{R}$.

1. if $f$ is h-transnormal, then it is F-transnormal, 
2. if $f$ is $F$-transnormal with $F(\nabla f)=\mathfrak{b}(f)$ and $\tilde{\mathfrak{b}}, \mathfrak{b} \in C^{2}(M)$, then it is h-transnormal. In particular

$$
\|\bar{\nabla} f\|+\tilde{\mathfrak{b}} \circ f=F(\nabla f) .
$$

Proof. First observe that $\nabla f=0$ if and only if $\bar{\nabla} f=0$. Suppose that we are in the regular part. Using Corollary 2.2.9,

$$
\bar{\nabla} f+\|\bar{\nabla} f\| W=\frac{\|\bar{\nabla} f\|}{F(\nabla f)} \nabla f
$$

Hence,

$$
\begin{aligned}
h(\bar{\nabla} f, \bar{\nabla} f)+\|\bar{\nabla} f\| h(W, \bar{\nabla} f) & =\frac{\|\bar{\nabla} f\|}{F \nabla f} h(\nabla f, \bar{\nabla} f)=\frac{\|\bar{\nabla} f\|}{F(\nabla f)} d f(\nabla f) \\
& =\frac{\|\bar{\nabla} f\|}{F(\nabla f)} g_{\nabla f}(\nabla f, \nabla f)=\frac{\|\bar{\nabla} f\|}{F(\nabla f)} F^{2}(\nabla f) \\
& =\|\bar{\nabla} f\| F(\nabla f) .
\end{aligned}
$$

So

$$
\|\bar{\nabla} f\|+h(W, \bar{\nabla} f)=F(\nabla f) .
$$

Applying the hypothesis we get $\|\bar{\nabla} f\|+\tilde{\mathfrak{b}} \circ f=F(\nabla f)$. Using this equality the proof of both items are at hand.

Remark 2.2.13. Observe that EQ. 2.7 in Lemma 2.2.12 provides us a formula to calculate $F(\nabla f)$ is the case of Randers using the Riemannian metric $h$ and $\bar{\nabla} f$.

\subsubsection{Geodesics in Randers spaces}

Shen in [She01a] showed that given a time independent wind $W$ on a Riemannian space $(M, h)$, the trajectories that minimize the time of travel are exactly the geodesics of the associated Randers metric. Assume that we are given the Zermelo's data $(M, h ; W)$ with the associated Randers metric $F$ such that $W$ is a Killing vector field. It is worth mentioning that in this case there is a relation which related the geodesics in the Randers space $(M, F)$ to the geodesics in the Riemannian space $(M, h)$ which is given below. Indeed, in order to find the $F$-geodesics that is enough to compose the flow of $W$ with the $h$-geodesics. So we have the following lemma that the proof is given in [Rob07].

Lemma 2.2.14. Given a geodesic $\gamma:(-\epsilon, \epsilon) \longrightarrow M$ of $(M, h)$ and a Killing vector field $W$, the unitary geodesics of $(M, F)$ are $\tilde{\gamma}(t)=\varphi(t, \gamma(t))$, where: $\varphi:(-\epsilon, \epsilon) \times U \longrightarrow M$ is the flow of $W$ through $\gamma(0)$.

In Picture 2.4 both geodesics $\gamma(t)$ and $\tilde{\gamma}(t)$ for two special cases are shown. Note that $\tilde{\gamma}(0)=$ $\varphi(0, \gamma(0))=\gamma(0)$ which means both geodesics have the same initial points.

\subsection{Randers-Minkowski space}

Let $\alpha$ be an Euclidean norm on $V$ and $\beta$ be a linear 1-form on $V$. Define $F(y):=\alpha(y)+\beta(y)$, for $y \in V$. Clearly $F$ is smooth on $V \backslash\{0\}$ and $F(\lambda y)=\lambda F(y)$, for every $\lambda>0$. By some calculations one can show that $g_{y}$ defined as 1.1 is linear, symmetric, and positive definite if and only if $\|\mid \beta\|<1$ 

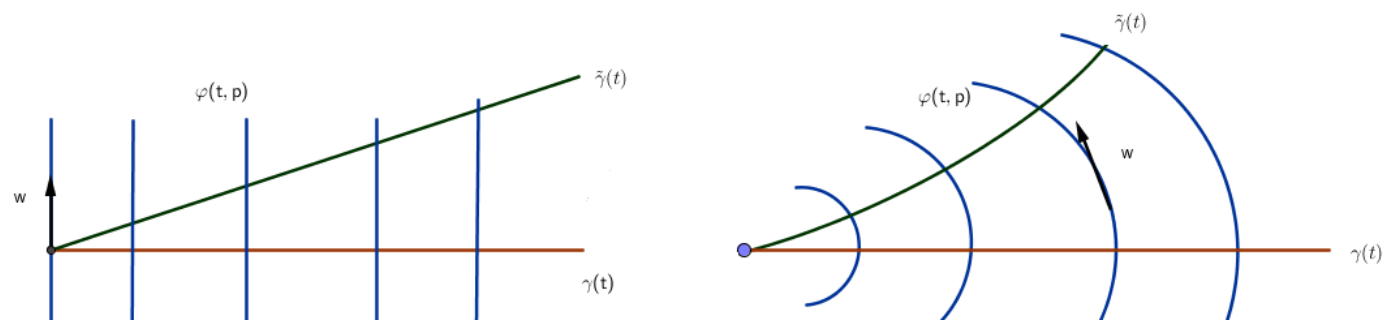

Figure 2.4: the composition of the geodesics with the flow of the wind

in which

$$
\|\beta\|:=\sup _{\alpha(y)=1} \beta(y)=\sqrt{\sum_{i, j=1}^{n} a^{i j} b_{i} b_{j}}
$$

where for a fixed basis $\left\{e_{i}\right\}_{i=1}^{n}$,

$$
y=\sum_{i=1}^{n} y^{i} e_{i}, \quad \alpha(y)=\sqrt{\sum_{i, j=1}^{n} a_{i j} y^{i} y^{j}}, \beta(y)=\sum_{i=1}^{n} b_{i} y^{i}
$$

and

$$
\left(a^{i j}\right)=\left(a_{i j}\right)^{-1} .
$$

This norm is called a Randers-Minkowski norm. A Randers-Minkowski space is a Randers space in which the metric does not depend on the point. So the wind must be constant $(W=$ cte. $)$. Indeed a Randers-Minkowski space is obtained by putting a constant wind on a Minkowski space. As a consequence the indicatrices in a Randers-Minkowski space $(M, F)$ are just translations of the geometric sphere in space $(V, \| .||)$. Moreover, since the geodesics in a Minkowski space are straight lines, according to Lemma 2.2.14, the geodesics in the associate Randers-Minkowski space are still straight lines. Let us see an example of a Randers-Minkowski space.

Example 2.3.1. Consider the canonical metric $h(u, v)=u_{1} v_{1}+u_{2} v_{2}$ on $\mathbb{R}^{2}$. Assume that we have a constant wind $W=\left(0, \frac{1}{2}\right)$ on $\mathbb{R}^{2}$. First, by Proposition 2.2.2 and some straightforward calculations one gets, for $v \in \mathbb{R}^{2}$,

$$
F(v)=\frac{2}{3}\left[\sqrt{3 v_{1}^{2}+4 v_{2}^{2}}-v_{2}\right] .
$$

Also for the sake of curiosity one can also calculate $a(u, v)=\frac{4}{3} u_{1} v_{1}+\frac{16}{9} u_{2} v_{2}$ and $\beta(v)=-\frac{2}{3} v_{2}$. According to Lemma 2.2.14 for a geodesic $\gamma(t)=p+t v$ in the Euclidean space $\mathbb{R}^{2}$ the corresponding $F$-geodesic is $\tilde{\gamma}(t)=p+t(v+W)$. Indeed for $\gamma(t)=\left(x_{0}, y_{0}\right)+t\left(v_{1}, v_{2}\right)$, we have

$$
\tilde{\gamma}(t)=\varphi(t,(\gamma(t)))=\left(x_{0}, y_{0}\right)+t\left(v_{1}, v_{2}+\frac{1}{2}\right) .
$$

Here $\varphi(t,(x, y))=\left(x, \frac{1}{2} t+y\right)$ is the flow of $W=\left(0, \frac{1}{2}\right)$. So the geodesics $\gamma_{1}(t)=p+t(p-W)$ and $\gamma_{2}(t)=p+t(-p+W)$ which coincide in $\mathbb{R}^{2}$ give the geodesics $\tilde{\gamma}_{1}(t)=p+t p$ and $\tilde{\gamma}_{2}(t)=$ $p+t(-p+2 W)$, respectively, which are two different F-geodesics. The geodesics in both spaces for some points are given in Table 2.1. 
Table 2.1: Geodesic

\begin{tabular}{|c|c|c|}
\hline$p$ & $\gamma_{1}, \gamma_{2}$ & $\tilde{\gamma}_{1}, \tilde{\gamma}_{2}$ \\
\hline$\left(1, \frac{1}{2}\right)$ & $\begin{array}{l}\left(1, \frac{1}{2}\right)+t(1,0) \\
\left(1, \frac{1}{2}\right)+t(-1,0) \\
\end{array}$ & $\begin{array}{l}\left(1, \frac{1}{2}\right)+t\left(1, \frac{1}{2}\right) \\
\left(1, \frac{1}{2}\right)+t\left(-1, \frac{1}{2}\right)\end{array}$ \\
\hline$\left(\frac{\sqrt{2}}{2}, \frac{1+\sqrt{2}}{2}\right)$ & $\begin{array}{l}\left(\frac{\sqrt{2}}{2}, \frac{1+\sqrt{2}}{2}\right)+t\left(\frac{\sqrt{2}}{2}, \frac{\sqrt{2}}{2}\right) \\
\left(\frac{\sqrt{2}}{2}, \frac{1+\sqrt{2}}{2}\right)+t\left(-\frac{\sqrt{2}}{2},-\frac{\sqrt{2}}{2}\right)\end{array}$ & $\begin{array}{l}\left(\frac{\sqrt{2}}{2}, \frac{1+\sqrt{2}}{2}\right)+t\left(\frac{\sqrt{2}}{2}, \frac{1+\sqrt{2}}{2}\right) \\
\left(\frac{\sqrt{2}}{2}, \frac{1+\sqrt{2}}{2}\right)+t\left(-\frac{\sqrt{2}}{2}, \frac{1-\sqrt{2}}{2}\right)\end{array}$ \\
\hline$\left(\frac{\sqrt{2}}{2}, \frac{1-\sqrt{2}}{2}\right)$ & $\begin{array}{l}\left(\frac{\sqrt{2}}{2}, \frac{1-\sqrt{2}}{2}\right)+t\left(\frac{\sqrt{2}}{2},-\frac{\sqrt{2}}{2}\right) \\
\left(\frac{\sqrt{2}}{2}, \frac{1-\sqrt{2}}{2}\right)+t\left(-\frac{\sqrt{2}}{2}, \frac{\sqrt{2}}{2}\right)\end{array}$ & $\begin{array}{l}\left(\frac{\sqrt{2}}{2}, \frac{1-\sqrt{2}}{2}\right)+t\left(\frac{\sqrt{2}}{2}, \frac{1-\sqrt{2}}{2}\right) \\
\left(\frac{\sqrt{2}}{2}, \frac{1-\sqrt{2}}{2}\right)+t\left(-\frac{\sqrt{2}}{2}, \frac{1+\sqrt{2}}{2}\right)\end{array}$ \\
\hline$\left(-1, \frac{1}{2}\right)$ & $\begin{array}{l}\left(-1, \frac{1}{2}\right)+t(-1,0) \\
\left(-1, \frac{1}{2}\right)+t(1,0)\end{array}$ & $\begin{array}{l}\left(-1, \frac{1}{2}\right)+t\left(-1, \frac{1}{2}\right) \\
\left(-1, \frac{1}{2}\right)+t\left(1, \frac{1}{2}\right)\end{array}$ \\
\hline$\left(-\frac{\sqrt{2}}{2}, \frac{1+\sqrt{2}}{2}\right)$ & $\begin{array}{l}\left(-\frac{\sqrt{2}}{2}, \frac{1+\sqrt{2}}{2}\right)+t\left(-\frac{\sqrt{2}}{2}, \frac{\sqrt{2}}{2}\right) \\
\left(-\frac{\sqrt{2}}{2}, \frac{1+\sqrt{2}}{2}\right)+t\left(\frac{\sqrt{2}}{2},-\frac{\sqrt{2}}{2}\right)\end{array}$ & $\begin{array}{l}\left(-\frac{\sqrt{2}}{2}, \frac{1+\sqrt{2}}{2}\right)+t\left(-\frac{\sqrt{2}}{2}, \frac{1+\sqrt{2}}{2}\right) \\
\left(-\frac{\sqrt{2}}{2}, \frac{1+\sqrt{2}}{2}\right)+t\left(\frac{\sqrt{2}}{2}, \frac{1-\sqrt{2}}{2}\right)\end{array}$ \\
\hline$\left(-\frac{\sqrt{2}}{2}, \frac{1-\sqrt{2}}{2}\right)$ & $\begin{array}{l}\left(-\frac{\sqrt{2}}{2}, \frac{1-\sqrt{2}}{2}\right)+t\left(-\frac{\sqrt{2}}{2},-\frac{\sqrt{2}}{2}\right) \\
\left(-\frac{\sqrt{2}}{2}, \frac{1-\sqrt{2}}{2}\right)+t\left(\frac{\sqrt{2}}{2}, \frac{\sqrt{2}}{2}\right)\end{array}$ & $\begin{array}{l}\left(-\frac{\sqrt{2}}{2}, \frac{1-\sqrt{2}}{2}\right)+t\left(-\frac{\sqrt{2}}{2}, \frac{1-\sqrt{2}}{2}\right) \\
\left(-\frac{\sqrt{2}}{2}, \frac{1-\sqrt{2}}{2}\right)+t\left(\frac{\sqrt{2}}{2}, \frac{1+\sqrt{2}}{2}\right)\end{array}$ \\
\hline
\end{tabular}

\subsection{On Finsler foliation in Randers spaces}

As it is seen in the definition of a SFF in Chapter 1, we are involved in the cone. In particular in the special case of a Randers metric

$$
F(y)=\sqrt{\frac{h^{2}(y, W)+\lambda h(y, y)}{\lambda^{2}}}-\frac{h(y, W)}{\lambda},
$$

according to Lemma 2.2.6, we have that $g_{y}(y, u)=0$ if and only if $h(u, y-F(y) W)=0$. That is given a submanifold $L \subset M, y \in \mathfrak{C}_{p}$ if and only if $\frac{y}{F(y)}-W$ is orthogonal to the leaf with respect to the Riemannian metric $h$. If also $F(y)=1$ we have $y \in \mathfrak{C}_{p}$ if and only if $y-W$ is orthogonal to $L$ with respect to the Riemannian metric $h$. In Chapter 1 Example 1.4.8 is a SFF. As we promised

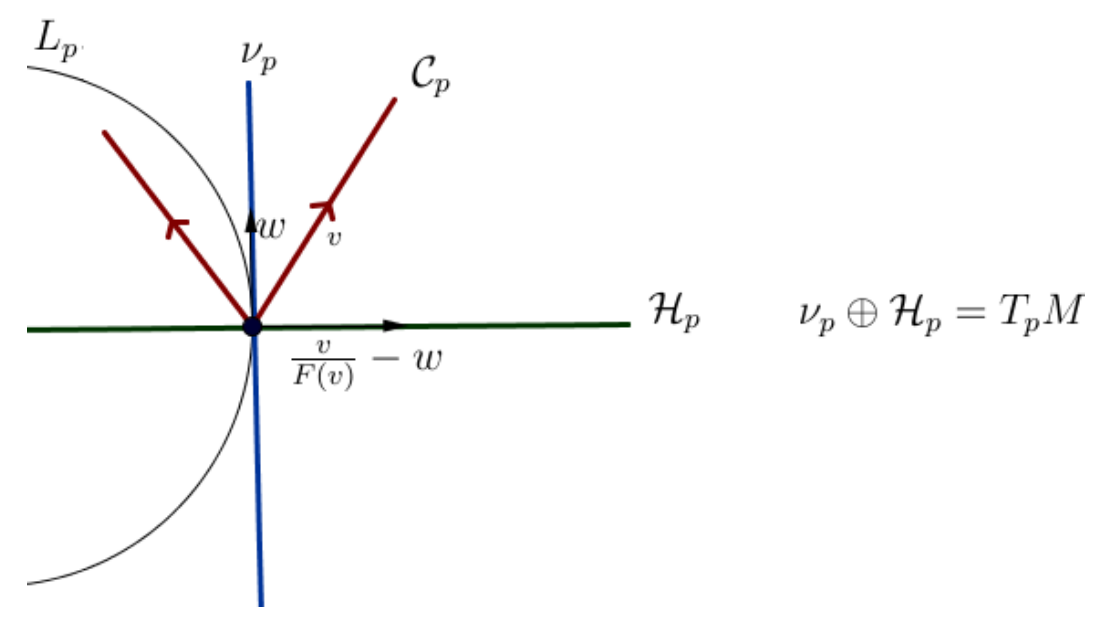

Figure 2.5: Decomposition of the space

we shall continue with an example of a singular foliation that it is not a SFF.

Example 2.4.1. We recall Example 2.3.1 and define $\rho_{+}: \mathbb{R}^{2} \longrightarrow \mathbb{R}$ with $\rho_{+}(p)=d_{F}(0, p)$. Notice that as in the Minkowski spaces the geodesics are the straight lines, for the geodesic $\gamma(t)=p t$ we 
have

$$
d_{F}(0, p)=\int_{0}^{1} F\left(\gamma^{\prime}\right)=F\left(\gamma^{\prime}\right)=F(p)
$$

Hence

$$
\rho_{+}^{-1}(r)=\left\{p \in \mathbb{R}^{2} \mid d_{F}(0, p)=r\right\}=\left\{p \in \mathbb{R}^{2} \mid F(p)=r\right\}=\Sigma_{r}^{F}=\Sigma_{r}^{h}+r W,
$$

where in the last equality we used Lemma 2.2.4. So we have a singular foliation $\mathcal{F}=\left\{\rho_{+}^{-1}(r)\right\}_{r \geq 0}$ by the circles in $\mathbb{R}^{2}$. Indeed

$$
\rho_{+}^{-1}(r)=\left\{p \in \mathbb{R}^{2} \mid p_{1}^{2}+\left(p_{2}-\frac{r}{2}\right)^{2}=r^{2}\right\}
$$

First let us find the cone $\mathfrak{C}_{p}$ for $p \in \Sigma^{F}$. With respect to the explanation before the example and in order to simplify the calculations we confine our attention to $v \in \mathfrak{C}_{p} \cap \Sigma^{F}(p)$, in which $\Sigma^{F}(p)$ is the unitary indicatrix centered at $p$. Hence

$$
v \in \mathfrak{C}_{p} \cap \Sigma^{F}(p) \text { if and only if } h\left(u, v-\left(0, \frac{1}{2}\right)\right)=0
$$

for every $u \in T_{p} \Sigma^{h}$. See the right hand picture of Figure 2.6 and in the left hand picture find the cones in some special points. Also in Table 2.2 some information for these points are provided. Here by $\mathbb{B}$

Table 2.2: Cone

\begin{tabular}{|l|l|l|l|}
\hline$p \in \Sigma^{F}$ & $p-W \in \Sigma^{h}$ & $\mathbb{B}$ & $\mathbb{G}$ \\
\hline$p_{1}:\left(1, \frac{1}{2}\right)$ & $(1,0)$ & $(0,1)$ & $\left(1, \frac{1}{2}\right)\left(-1, \frac{1}{2}\right)$ \\
$p_{2}:\left(\frac{\sqrt{2}}{2}, \frac{1+\sqrt{2}}{2}\right)$ & $\left(\frac{\sqrt{2}}{2}, \frac{\sqrt{2}}{2}\right)$ & $(-1,1)$ & $\left(\frac{\sqrt{2}}{2}, \frac{1+\sqrt{2}}{2}\right),\left(-\frac{\sqrt{2}}{2}, \frac{1-\sqrt{2}}{2}\right)$ \\
$p_{3}:\left(0, \frac{3}{2}\right)$ & $(0,1)$ & $(1,0)$ & $\left(0,-\frac{1}{2}\right),\left(0, \frac{3}{2}\right)$ \\
$p_{4}:\left(\frac{\sqrt{2}}{2}, \frac{1-\sqrt{2}}{2}\right)$ & $\left(\frac{\sqrt{2}}{2},-\frac{\sqrt{2}}{2}\right)$ & $(1,1)$ & $\left(\frac{\sqrt{2}}{2}, \frac{1-\sqrt{2}}{2}\right),\left(-\frac{\sqrt{2}}{2}, \frac{1+\sqrt{2}}{2}\right)$ \\
$p_{5}:\left(-\frac{\sqrt{2}}{2}, \frac{1+\sqrt{2}}{2}\right)$ & $\left(-\frac{\sqrt{2}}{2}, \frac{\sqrt{2}}{2}\right)$ & $(1,1)$ & $\left(-\frac{\sqrt{2}}{2}, \frac{1+\sqrt{2}}{2}\right),\left(\frac{\sqrt{2}}{2}, \frac{1-\sqrt{2}}{2}\right)$ \\
$p_{6}:\left(-1, \frac{1}{2}\right)$ & $(-1,0)$ & $(0,1)$ & $\left(-1, \frac{1}{2}\right)\left(1, \frac{1}{2}\right)$ \\
$p_{7}:\left(-\frac{\sqrt{2}}{2}, \frac{1-\sqrt{2}}{2}\right)$ & $\left(-\frac{\sqrt{2}}{2},-\frac{\sqrt{2}}{2}\right)$ & $(-1,1)$ & $\left(-\frac{\sqrt{2}}{2}, \frac{1-\sqrt{2}}{2}\right),\left(\frac{\sqrt{2}}{2}, \frac{1+\sqrt{2}}{2}\right)$ \\
$p_{8}:\left(0,-\frac{1}{2}\right)$ & $(0,-1)$ & $(1,0)$ & $\left(0,-\frac{1}{2}\right),\left(0, \frac{3}{2}\right)$ \\
\hline
\end{tabular}

we mean the basis of $T_{p} \Sigma^{F}(p)$ and by $\mathbb{G}$ we mean the generator of $\mathfrak{C}_{p}$ that is $\mathfrak{C}_{p}=\mathbb{R}^{+} . \mathbb{G}$. Comparing Table 2.2 with Table 2.1 we see that the velocities of geodesics $\tilde{\gamma}_{1}$ and $\tilde{\gamma}_{1}$ are the generators of $\mathfrak{C}_{p}$. Now observe that for the geodesic $\gamma(t)=\left(1, \frac{1}{2}\right)+t\left(-1, \frac{1}{2}\right)$ with respect to the table we have that

$$
\gamma^{\prime}(0) \in \mathfrak{C}_{\gamma(0)}\left(\Sigma_{1}^{F}\right) .
$$

Also $\gamma(1)=2 W=(0,1)$ and $F(0,1)=\frac{2}{3}$, so $\gamma(1) \in \Sigma_{\frac{2}{3}}^{F}$, but $\gamma^{\prime}(1) \notin \mathfrak{C}_{\gamma(1)}\left(\Sigma_{\frac{2}{3}}^{F}\right)$. In fact, $F\left(\gamma^{\prime}(1)\right)=1$ which means $\gamma^{\prime}(1) \in \Sigma^{F}(\gamma(1))$. Moreover remember that

$$
\gamma^{\prime}(1) \in \mathfrak{C}_{\gamma(1)}\left(\Sigma_{\frac{2}{3}}^{F}\right) \text { if and only if } h\left(u, \gamma^{\prime}(1)-W\right)=0
$$

for every $u \in T_{\gamma(1)-W} \Sigma_{r}^{h}$. However,

$$
h\left(u, \gamma^{\prime}(1)-W\right)=h\left((1,0),\left(-1, \frac{1}{2}\right)-\left(0, \frac{1}{2}\right)\right)=h((1,0),(-1,0))=-1 \neq 0 .
$$



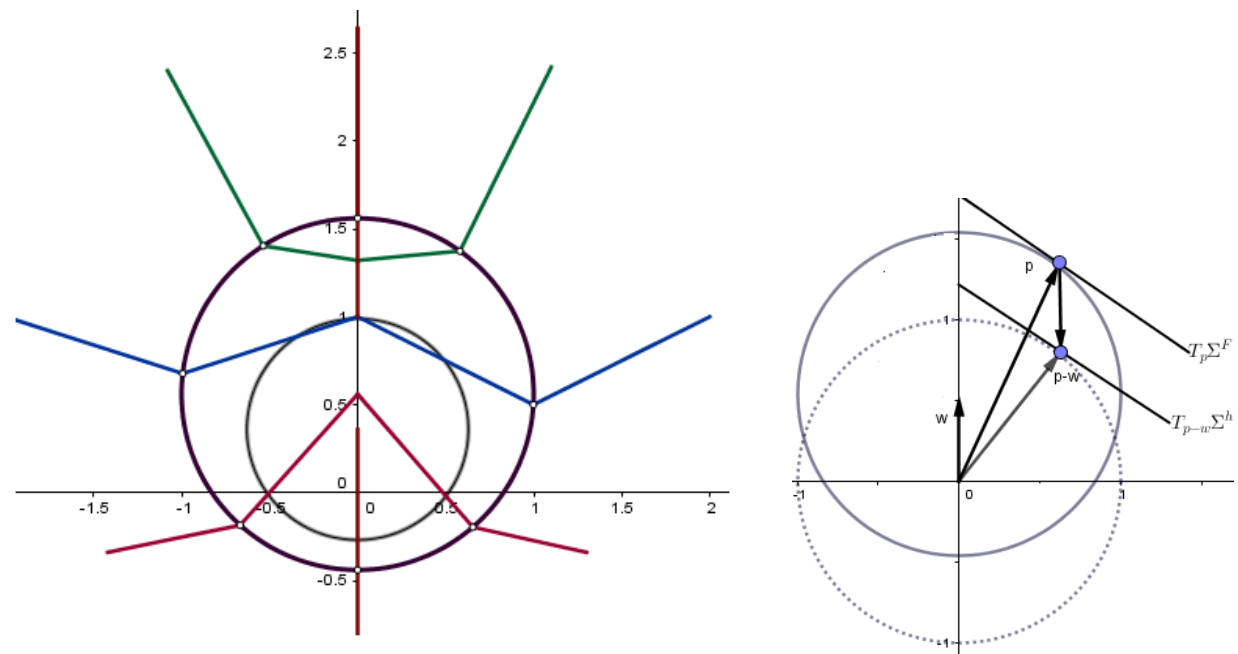

Figure 2.6: Cone and the tangent spaces at $p$ and $p-W$

These calculations mean that $\gamma^{\prime}(1)$ is not orthogonal to the indicatrix (leaf) while $\gamma^{\prime}(0)$ is. Although, generally without doing any calculation, it can be seen by knowing the fact that at the points $p$ belonging to the $y$-axis, the cones coincide with the $y$-axis. While for other points $p$ with the nonzero $x$-component, the orthogonal geodesic emanating from $p$ does not coincide with the $y$-axis at the point they intersect. To sum up, this foliation is not Finslerian.

Lemma 2.4.2. Given the Zermelo's data $(M, h ; W)$ with the associated Randers metric $F$ and a point $p \in L \subset M$, where $L$ is a submanifold,

$$
W_{p} \in \mathcal{H}_{p} \text { if and only if } \mathfrak{C}_{p}=\mathcal{H}_{p}
$$

Namely the wind at the point $p$ is orthogonal to the leaves with respect to $h$ if and only if the cone at this point coincides with the horizontal space. In particular the cone is a vector space.

Proof. Assume that $W_{p} \in \mathcal{H}_{p}$ and $v$ is a unitary vector. Considering the relation

$$
v \in \mathfrak{C}_{p} \text { if and only if } h(u, v-W)=0, \text { for every } u \in \nu_{p},
$$

leads to the fact that $\mathfrak{C}_{p} \equiv \mathcal{H}_{p}$. Now suppose that the cone $\mathfrak{C}_{p}$ coincides with $\mathcal{H}_{p}$ at a point $p$. Again the fact that

$$
v \in \mathfrak{C}_{p} \equiv \mathcal{H}_{p} \text { if and only if } v-W \in \mathcal{H}_{p}
$$

implies that $W \in \mathcal{H}_{p}$.

Corollary 2.4.3. If we have a Randers singular foliation with $W \in \mathcal{H}$, then it is a SRF.

\subsubsection{Examples}

In this part some examples of Randers spaces are provided. The goal is to study the foliation on these spaces. So we first consider a foliation of the spaces. Then putting a wind, the associated Randers metric is calculated. Then we study the geodesics in both, the Riemannian and the associated Randers space and we check if the foliation is a Finslerian one or not. Some of the geometric objects are also calculated. 
Example 2.4.4. Consider the Euclidean space $\left(\mathbb{R}^{n+1}, h\right), n>1$, and let $D_{s}=\left\{(x, y) \in \mathbb{R}^{n+1} \mid\|x\|^{2}+\right.$ $\left.y^{2}<s^{2}\right\}$, for some $s>0$, with the foliation

$$
\mathcal{F}=\left\{(x, y) \in \mathbb{R}^{n} \times \mathbb{R}:\|x\|^{2}=r\right\}
$$

of $D_{s}$ consists of the cylinders of radius $r<s$ around the $y$-axis. Indeed one can define $f: D_{s} \longrightarrow \mathbb{R}$ with $f(x, y)=\|x\|^{2}$. Then $\mathcal{F}=\left\{f^{-1}(r)\right\}_{r \geq 0}$. Now put the wind

$$
W(x, y)=\frac{(x, 0)}{s}
$$

on it and denote the Randers metric associated to it with $F$. We claim that $\left(\mathbb{R}^{n}, F, \mathcal{F}\right)$ is a $\boldsymbol{S F F}$. First as $W_{p} \in \mathcal{H}_{p}$ by Lemma 2.4.2 we have that $\mathfrak{C}_{p} \equiv \mathcal{H}_{p}$. Moreover a geodesic $\gamma(t)$ is orthogonal to a leaf $L_{p}$ at a point $p=(x, y) \in L_{p}$ (with respect to $h$ ) if and only if $\gamma^{\prime}(t)=\lambda(x, 0)$ for some $\lambda \in \mathbb{R}$. Also note that, as the flow of $W$ consists of the lines (orthogonal to $y$-axis), then the geodesics of $\left(\mathbb{R}^{n+1}, F\right)$ are still the straight lines. As a result the geodesics $\gamma$ are orthogonal to all the leaves they meet and so $\mathcal{F}$ is $\boldsymbol{a} \boldsymbol{S F F}$ and also a $\boldsymbol{S R F}$.

For the sake of curiosity, let us calculate the gradient and the Hessian for some special cases. First as $W$ at each point is orthogonal to the leaves, by Lemma 2.2.12 we have

$$
\|\bar{\nabla} f\|+h(W, \bar{\nabla} f)=F(\nabla f) .
$$

With some calculations one gets $\bar{\nabla} f(x, y)=2(x, 0),\|\bar{\nabla} f\|^{2}=4 f$, and $h(\bar{\nabla} f, W)=\frac{2\|x\|^{2}}{s}=\frac{2 f}{s}$ that leads to

$$
F(\nabla f)=2 \sqrt{f}+\frac{2 f}{s}
$$

which also means that $f$ is $F$ - transnormal with $\mathfrak{b}(t)=\left(2 \sqrt{t}+\frac{2 t}{s}\right)^{2}$. Next using 2.2.9,

$$
\nabla f=\left(2 \sqrt{f}+\frac{2 f}{s}\right)\left(\frac{(x, 0)}{s}+\frac{2(x, 0)}{2 \sqrt{f}}\right) .
$$

Furthermore, according to Definition 1.3 .36 and Lemma 1.3.41,

$$
\operatorname{Hess} f\left(0, x_{n+1}\right)=2 I_{n+1} \text { and Hess } f(\nabla f, \nabla f)=\frac{\mathfrak{b}(f) \mathfrak{b}^{\prime}(f)}{2}
$$

which are, resp., the Hess in the critical points and Hess in the regular points and along the gradient vector.

Example 2.4.5. Consider the space $\left(D_{s} \backslash 0, h\right)$ where $D_{s}=\left\{(x, y) \in \mathbb{R}^{2} \mid x^{2}+y^{2}<s^{2}\right\}$ and $h$ is the canonical metric and $f: D_{s} \backslash 0 \longrightarrow \mathbb{R}$ with $f(x, y)=x^{2}+y^{2}$, that is the square of the distance function in $\left(D_{s} \backslash 0, h\right)$. So we have a foliation of $\mathbb{R}^{2} \backslash 0$ as the circles around the origin. Now consider the wind

$$
W(x, y)=\frac{(x, y)}{s} .
$$

Then we have a Randers space $\left(D_{s} \backslash 0, F\right)$. Here $W$ is orthogonal to each leaf with respect to $h$ and so by Lemma 2.4.2, $\mathfrak{C}=\mathcal{H}$. As also the flow of $W$ consists of the lines (passing through the origin), then the geodesics of $\left(D_{s} \backslash 0, F\right)$ are still the straight lines. As the lines passing through the origin intersect all leaves orthogonally we are faced with a SFF which is also a SRF. Let us calculate 
$F(\nabla f)$ and the Hessian in some special cases. Note that $\bar{\nabla} f=2(x, y)$ and so $h(W, \bar{\nabla} f)=\frac{2}{s} f$. Then one can apply Lemma 2.2.12 in which $\tilde{\mathfrak{b}}: \mathbb{R} \longrightarrow \mathbb{R}$ is as $\tilde{\mathfrak{b}}(t)=t$. Using this lemma we have $\|\bar{\nabla} f\|+\sqrt{f}=F(\nabla f)$ which means

$$
2 \sqrt{f}+\frac{2}{s} f=F(\nabla f)
$$

So $f$ is F-transnormal with $\mathfrak{b}(t)=\left(2 \sqrt{f}+\frac{2}{s} f\right)^{2}$. Moreover, according to Lemma 1.3.41 we have

$$
\text { Hess } f(\nabla f, \nabla f)=\left(2 \sqrt{f}+\frac{2}{s} f\right)^{2}\left(\frac{1}{\sqrt{f}}+\frac{2}{s}\right)
$$

One can use Lemma 1.3.41 to obtain the gradient vector and also by Definition 1.3.36,

$$
\operatorname{Hess} f(0)=2 I_{2} \text {. }
$$

Example 2.4.6. [Rob07] We will proceed with providing this type of Randers metrics on the sphere arising as solutions to Zermelo's problem of navigation on the canonical Riemannian sphere $\left(S^{n}, h\right)$, under the influence of a Killing vector field $W$. Some special geodesics on the Riemannian sphere are considered and then putting some special winds, the behavior of the corresponding Randers geodesics are studied. We are interested to see how many geodesic close at each case. Observe that in the Riemannian sphere $\left(S^{n}, h\right)$ each geodesic closes. Although for some Randers spaces it is not the case and there are infinite many geodesics that never close. The Randers metrics on the sphere $S^{n}$ were introduced by Katok in 1973 [Kat73] and then studied by Ziller in 1983 [Zil83]. Let's start constructing our example. A Killing vector field $W$ at each point is expressed, modulo an orthogonal transformation of $\mathbb{R}^{n+1}$, as

$$
W(x)=x \cdot A
$$

where $x=\left(x_{0}, \ldots, x_{n}\right) \in S^{n} \subset \mathbb{R}^{n+1}$ and $A$ is a $(n+1) \times(n+1)$ matrix of the following form

$$
A=\left\{\begin{array}{lll}
a_{1} J \oplus \ldots \oplus a_{k} J \oplus 0 & \text { if } & n=2 k \\
a_{1} J \oplus \ldots \oplus a_{k} J & \text { if } & n=2 k-1 .
\end{array}\right.
$$

Here

$$
J=\left(\begin{array}{cc}
0 & 1 \\
-1 & 0
\end{array}\right)
$$

and $a_{i}$ ordered such that $0 \leq a_{k} \leq \ldots \leq a_{2} \leq a_{1}<1$. The condition $a_{1}$ guarantees that $\|W\|<1$. Observe that the flow at each point is conjugate to a diagonal matrix $\operatorname{Rot}\left(a_{1} t, \ldots, a_{k} t\right)$, where

$$
\operatorname{Rot}\left(a_{1} t, \ldots, a_{k} t\right)=\left\{\begin{array}{lll}
R\left(a_{1} t\right) \oplus \ldots \oplus R\left(a_{k} t\right) \oplus 1 & \text { if } & n=2 k \\
R\left(a_{1} t\right) \oplus \ldots \oplus R\left(a_{k} t\right) & \text { if } & n=2 k-1,
\end{array}\right.
$$

and

$$
R\left(a_{i} t\right)=\left(\begin{array}{cc}
\cos a_{i} t & \sin a_{i} t \\
-\sin a_{i} t & \cos a_{i} t
\end{array}\right)
$$

and so the flow of such a field is given by $\varphi(t, x)=x \cdot \operatorname{Rot}\left(a_{1} t, \ldots, a_{k} t\right)$. Generally on $\left(S^{n}, F\right)$ $(n=2 k$ or $2 k-1)$ at least $2 k$ geodesic close. In the case that each $a_{i}$ is rational, all the 
geodesics of the Randers metric $F$ close. Assume that this is not the case. Set $a_{i}=\frac{a}{p_{i}}$, where $0<a<1$ is irrational and $p_{i}$ are relatively prime integers with $1<p_{1}<p_{2}<\ldots<p_{k}$. Ziller [Zil83] showed that such choices of $a_{i}$ give us Randers metrics for which only $2 k$ geodesics close. Observe that, unlike the Riemannian case, two geodesics that track the same path may be considered distinct. Because they have two different lengths (it will be shown below). Moreover, there exist geodesics that self-intersect however never close. According to Lemma 2.2.14, given a geodesic $\alpha$ of $\left(S^{n}, h\right)$, the corresponding Randers geodesic is given by $\gamma(t)=\varphi(t, \alpha(t))$.

We first confine our attention to those h-geodesics parameterizing the great circles which are invariant under the flow $\varphi(t,$.$) . Note that we have only k$ such geodesics which can be written in the following form

$$
\alpha_{i}=\left\{x \in S^{n} \mid x^{j}=0, \forall j \neq 2 i-2,2 i-1\right\}, i=1, \ldots, k .
$$

Let us rewrite these geodesics and investigate them a bit more. Indeed we have

$$
\begin{aligned}
& \text { If } n=2 k-1:\left\{\begin{array}{l}
\alpha_{1}=\left\{\left(x_{0}, x_{1}, 0, \ldots, 0\right) \in S^{n}\right\} \\
\alpha_{2}=\left\{\left(0,0, x_{2}, x_{3}, 0, \ldots, 0\right) \in S^{n}\right\} \\
\vdots \\
\alpha_{k}=\left\{\left(0, \ldots, 0, x_{2 k-2}, x_{2 k-1}\right) \in S^{n}\right\}
\end{array}\right. \\
& \text { If } n=2 k:\left\{\begin{array}{l}
\alpha_{1}=\left\{\left(x_{0}, x_{1}, 0, \ldots, 0\right) \in S^{n}\right\} \\
\alpha_{2}=\left\{\left(0,0, x_{2}, x_{3}, 0, \ldots, 0\right) \in S^{n}\right\} \\
\vdots \\
\alpha_{k}=\left\{\left(0, \ldots, 0, x_{2 k-2}, x_{2 k-1}, 0\right) \in S^{n}\right\}
\end{array}\right.
\end{aligned}
$$

It means the corresponding F-geodesic $\gamma_{i}$ is simply a reparametrization of $\alpha_{i}$, and of course with the same trace. Although for two geodesics that have the same trace, but different directions, the lengths may not be identical. In fact the geodesic $\gamma_{i}$ that is in the direction of the rotation $R\left(a_{i} t\right)$ has shorter length than $\alpha_{i}$, that is $L\left(\gamma_{i}\right)=\frac{2 \pi}{1+a_{i}}$, and otherwise it has longer length than $\alpha_{i}$, that is $L\left(\gamma_{i}\right)=\frac{2 \pi}{1-a_{i}}$.

4 Let us examine certain of these geodesics on a more familiar case that is $S^{2}$ with the corresponding Randers metric associated to

$$
W_{x}=x \cdot\left(\begin{array}{ccc}
0 & a & 0 \\
-a & 0 & 0 \\
0 & 0 & 0
\end{array}\right)
$$

where $0 \leq a<1$ and whose flow

$$
\varphi(t, x)=x .\left(\begin{array}{ccc}
\cos a t & \sin a t & 0 \\
-\sin a t & \cos a t & 0 \\
0 & 0 & 1
\end{array}\right) .
$$


Consider the geodesics $\alpha_{1}^{i}(t)$ as two different parametrizations of geodesic $\alpha_{1}$ addressed in EQ. 2.10 and whose associated Randers geodesics $\gamma_{1}^{i}(t)$ as following:

$$
\begin{aligned}
& \alpha_{1}^{1}(t)=(\cos t, \sin t, 0) \quad ; \quad \gamma_{1}^{1}(t)=(\cos t \cos a t-\sin t \sin a t, \cos t \sin a t+\sin t \cos a t, 0) \\
& \alpha_{1}^{2}(t)=(\sin t, \cos t, 0) \quad ; \quad \gamma_{1}^{2}(t)=(\sin t \cos a t-\cos t \sin a t, \sin t \sin a t+\cos t \cos a t, 0) .
\end{aligned}
$$

Observe that $\alpha_{1}^{i}$ s are the same geodesics, up to a reparametrization, and so they have the same trace which coincide with the unit circle in the $x y$-plane and so $\gamma_{1}^{i} s$ do. Although the wind $W$ affects the velocity in these geodesics and so the length. Let's be a bit more specific and see the Randers metric for the case in which

$$
W_{x}=x .\left(\begin{array}{ccc}
0 & \frac{1}{2} & 0 \\
-\frac{1}{2} & 0 & 0 \\
0 & 0 & 0
\end{array}\right)
$$

In the following two pictures the geodesics 2.11, for $a=\frac{1}{2}$, are drawn. Also it is shown that after passing $\frac{\pi}{2}$ where each geodesic arrives. As it was mentioned above the geodesic in the same direction of the wind is faster and so shorter and the geodesic in the opposite direction of the wind gives slower and so longer geodesic. Observe that $L\left(\gamma_{1}^{1}\right)=\frac{2 \pi}{1+\frac{1}{2}}$ and $L\left(\gamma_{1}^{2}\right)=\frac{2 \pi}{1-\frac{1}{2}}, L\left(\alpha_{1}^{1}\right)=L\left(\alpha_{1}^{1}\right)=2 \pi$.
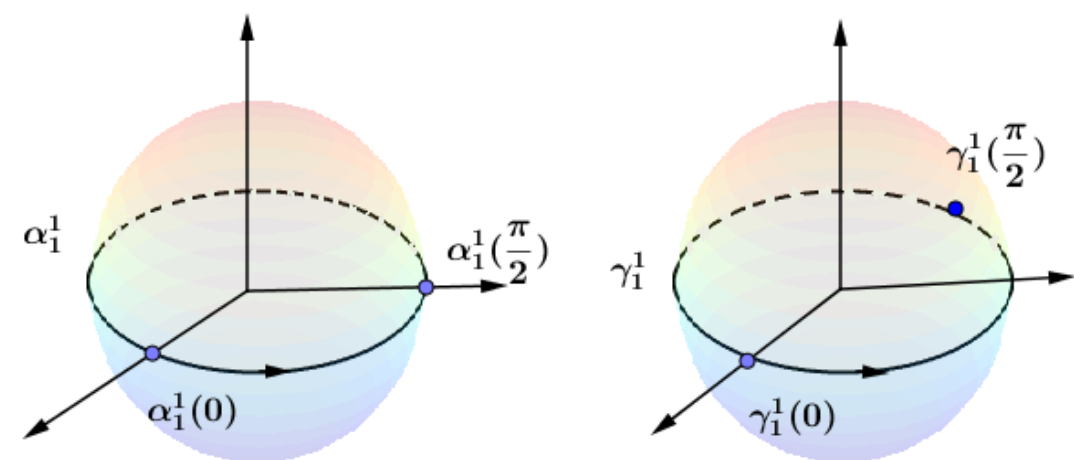

Figure 2.7
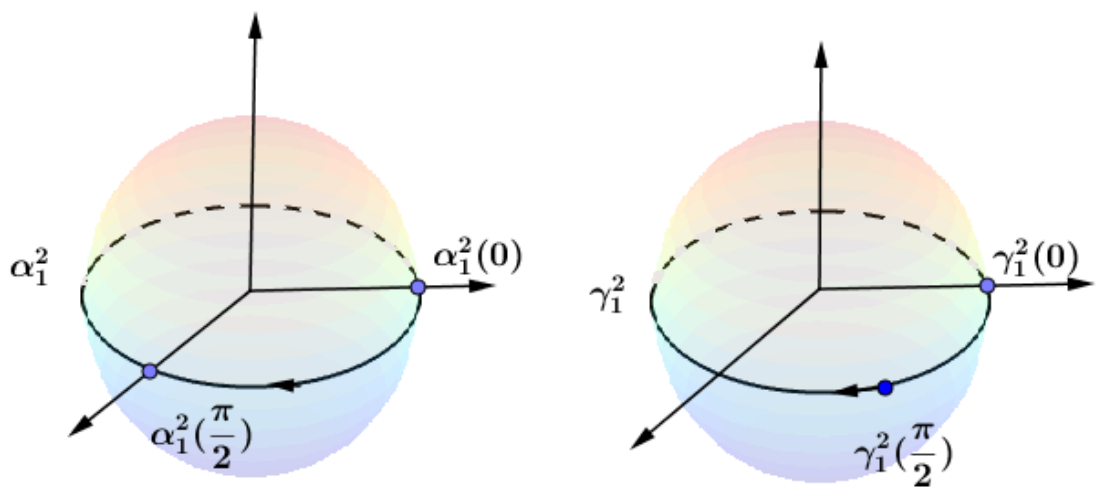

Figure 2.8 
Now consider the the case in which

$$
W_{x}=x\left(\begin{array}{ccc}
0 & \frac{1}{\sqrt{2}} & 0 \\
-\frac{1}{\sqrt{2}} & 0 & 0 \\
0 & 0 & 0
\end{array}\right)
$$

As it was mentioned before, we have just two choices of such geodesics, which are the same hgeodesics, but distinct F-geodesics. Also for geodesics 2.11, with $\frac{1}{\sqrt{2}}$, we have $L\left(\gamma_{1}^{1}\right)=\frac{2 \pi}{1+\frac{1}{\sqrt{2}}}$ and $L\left(\gamma_{1}^{2}\right)=\frac{2 \pi}{1-\frac{1}{\sqrt{2}}}, L\left(\alpha_{1}^{1}\right)=L\left(\alpha_{1}^{2}\right)=2 \pi$.

The other group of geodesics are the geodesics which are not invariant under the flow $\varphi(t,$.$) .$ One case would be the geodesics $\alpha$ and whose reparametrization $\tilde{\alpha}$ with the corresponding F-geodesics as follows

$$
\begin{aligned}
& \alpha(t)=(\sin t, 0, \ldots, 0, \cos t) \quad ; \quad \gamma(t)=\left(\sin t \cos a_{1} t, \sin t \sin a_{1} t, 0, \ldots, 0, \cos t\right) \\
& \tilde{\alpha}(t)=(\cos t, 0, \ldots, 0, \sin t) \quad ; \quad \tilde{\gamma}(t)=\left(\cos t \cos a_{1} t, \cos t \sin a_{1} t, 0, \ldots, 0, \sin t\right)
\end{aligned}
$$

4 Let us see some special cases:

case 1. $a_{1}=\frac{1}{2}$. In this case we have

$$
\left\{\begin{array}{l}
\gamma(0)=\gamma(2 \pi)=\gamma(4 \pi)=(0, \ldots, 0,1) \\
\tilde{\gamma}(0)=\tilde{\gamma}(2 \pi)=\tilde{\gamma}(4 \pi)=(1,0, \ldots, 0) \\
\gamma^{\prime}(0)=\gamma^{\prime}(4 \pi)=(1,0, \ldots, 0) \neq \gamma^{\prime}(2 \pi)=(-1,0, \ldots, 0) \\
\tilde{\gamma}^{\prime}(0)=\tilde{\gamma}^{\prime}(4 \pi)=\left(0, \frac{1}{2}, 0, \ldots, 0,1\right) \neq \tilde{\gamma}^{\prime}(2 \pi)=\left(0,-\frac{1}{2}, 0, \ldots, 0,1\right)
\end{array}\right.
$$

These calculations mean that $\gamma$ first self-intersects at $t=2 \pi$ but does not close. It selfintersects again at time $t=4 \pi$ in which it closes. One can say the same things for $\tilde{\gamma}$. Indeed for every $a_{1}$ rational such a geodesic $\gamma$ closes at time $t=\frac{2 \pi}{a_{1}}$. Notice that each geodesic is associate to a different Randers metric which varies as a varies and so we have infinite many Randers metrics. In Picture 2.9, $\alpha$ and $\gamma$ for $n=2$ are shown. The other geodesics have the same trace but different directions. Picture 2.10 is the top view of $\alpha$ and $\gamma(\tilde{\alpha}$ and $\tilde{\gamma})$. In this case once the geodesic $\gamma$ passes the sphere for the first time, $\alpha$ has passes the sphere 4 times.
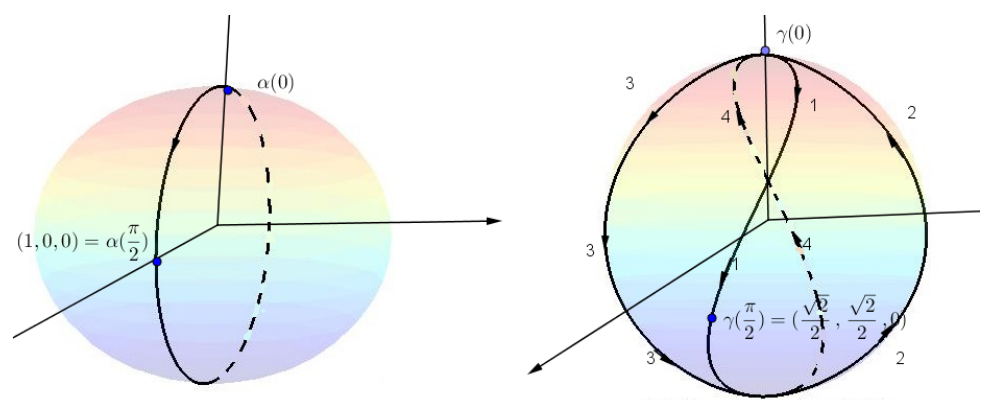

Figure 2.9

case 2. $a_{1}=\frac{1}{4}$. $\gamma$ self-intersects at timest $=0,2 \pi,, 4 \pi, 8 \pi$, although $\gamma^{\prime}(0)=\gamma^{\prime}(8 \pi) \neq \gamma^{\prime}(2 \pi) \neq \gamma^{\prime}(4 \pi)$. The geodesic $\gamma$ for $n=2$, from two different views, is shown in Picture 2.11. In this case once this geodesic passes the sphere for the first time, $\alpha$ has passes the sphere 8 times. 

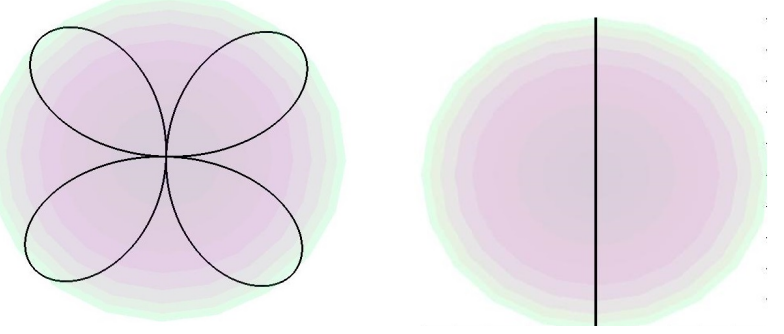

Figure 2.10
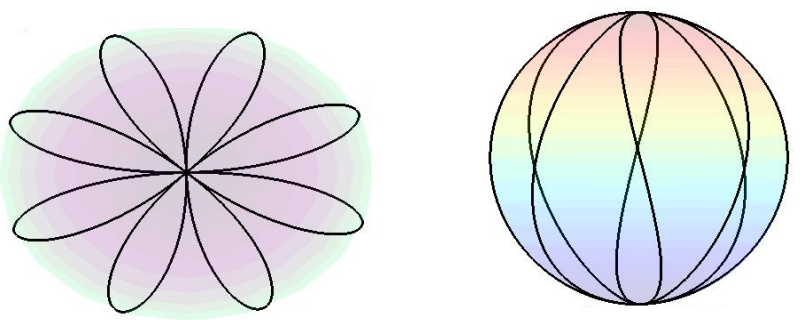

Figure 2.11

case 3. $a_{1}=\frac{2}{5}$. In this case once the geodesic $\gamma$ passes the sphere for the first time, $\alpha$ has passes the sphere 10 times. See Picture 2.12.
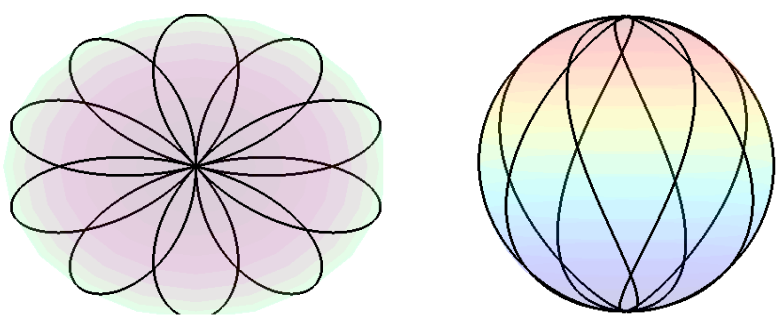

Figure 2.12

case 4. $a_{1}=\frac{7}{8}$. In this case once the geodesic $\gamma$ passes the sphere for the first time, $\alpha$ has passes the sphere 16 times. See Picture 2.13.
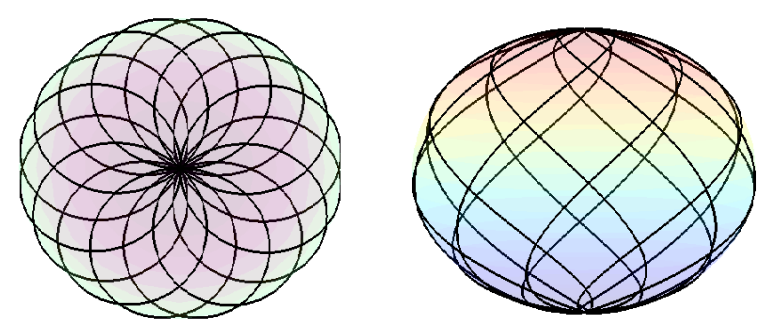

Figure 2.13

case 5. $a_{1}=\frac{1}{50}$. In this case once the geodesic $\gamma$ passes the sphere for the first time, $\alpha$ has passes the sphere 100 times. See Picture 2.14.

case 6. $a_{1}=\frac{1}{\sqrt{2}}$. Observe that in this case for every $l \in Z, \gamma(0)=\gamma(2 \pi l)$ however $\gamma^{\prime}(0) \neq \gamma^{\prime}(2 \pi l)$ which says that this geodesic never closes. See Picture 2.15 in which the geodesic $\gamma$ from the top for $t \in[0,100 \pi]$ (the left hand) and $t \in[0,1000 \pi]$, the right hand, is shown. 

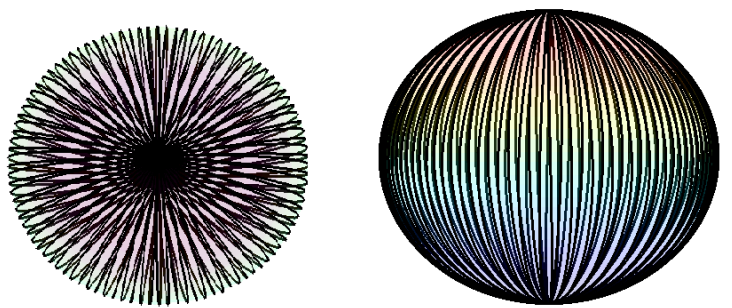

Figure 2.14

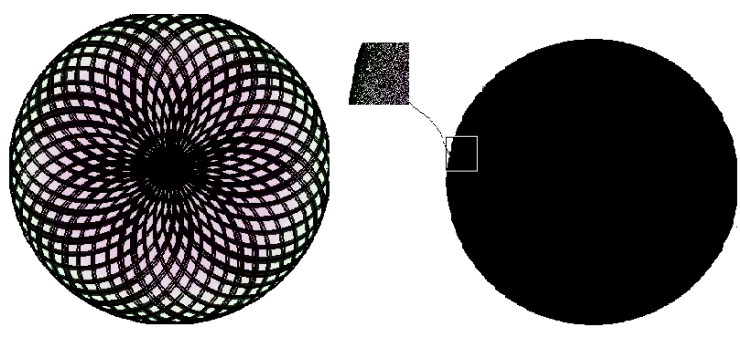

Figure 2.15

Now consider the function $f: \mathbb{R}^{n+1} \longrightarrow \mathbb{R}$ with $f(x)=\left(0, \ldots, 0, x_{n+1}\right)$ and define $\tilde{f}:=\left.f\right|_{S^{n}}$. Let us calculate $F(\nabla \tilde{f})$ in which $\nabla \tilde{f}$ is the gradient of $\tilde{f}$ with respect to $F$. Note that $W$ is tangent to $S^{n}$ and also tangent to the leaves. Because

$$
\operatorname{gradf}=\bar{\nabla} \tilde{f}+\langle\operatorname{gradf}, x\rangle x
$$

in which gradf is the gradient of $f$ with respect to the canonical metric $h, \bar{\nabla} \tilde{f}$ is the gradient of $\tilde{f}$ with respect to $\left.h\right|_{S^{n}}$, and $x \in S^{n}$. Therefore

$$
\bar{\nabla} \tilde{f}=(0, \ldots, 0,1)-\tilde{f} \cdot x
$$

which implies that $\langle\bar{\nabla} \tilde{f}, W\rangle=0$. Now one can apply Lemma 2.2.10 which says $F(\nabla \tilde{f})=\|\bar{\nabla} \tilde{f}\|$ and so it is sufficient to calculate $\|\bar{\nabla} \tilde{f}\|$. Since we wrote gradf as the orthogonal decomposition, we have

$$
\|\operatorname{gradf}\|^{2}=\|\bar{\nabla} \tilde{f}\|^{2}+\langle\operatorname{gradf}, x\rangle^{2}\|x\|^{2}
$$

that implies that $\|\bar{\nabla} \tilde{f}\|=\sqrt{1-\tilde{f}^{2}}$ which means for $\mathfrak{b}=1-t^{2}, F^{2}(\nabla \tilde{f})=\mathfrak{b} \circ \tilde{f}$. Also according to Lemma 2.2.10,

$$
\nabla \tilde{f}=\bar{\nabla} \tilde{f}+\|\bar{\nabla} \tilde{f}\| W=(0, \ldots, 0,1)-\tilde{f} \cdot x+\sqrt{1-\tilde{f}^{2}} W .
$$

It means that $\tilde{f}$ is a F-transnormal function. Note that we have a foliation as

$$
\mathcal{F}=\left.\left\{f^{-1}(r) \cap S^{n}\right\}\right|_{r \in[-1,1]}
$$

which we will see in Example 3.3.12 of Chapter 3, it is a $\mathbf{S F F}$ with some special properties. Observe that according to Lemma 1.3.41,

$$
\operatorname{Hess} \tilde{f}(\nabla \tilde{f}, \nabla \tilde{f})=-\tilde{f}(1-\tilde{f}) \text {. }
$$

In Example 3.4.8 it is shown that $\mathcal{F}$ is a $\boldsymbol{S R \boldsymbol { F }}$. 
Example 2.4.7. Consider the space $\left(\mathbb{R}^{n+1}, h\right)$ where $h$ is the canonical metric and let

$$
D_{s}=\left\{x \in \mathbb{R}^{n+1}:\|x\|<s\right\}
$$

for some $s>0$. Consider $f: \mathbb{R}^{n+1} \longrightarrow \mathbb{R}$ with $f(x)=\|x\|^{2}$, that is the square of the distance function in $\left(\mathbb{R}^{n+1}, h\right)$. So we have a foliation $\mathcal{F}=\left\{\Sigma_{r}^{h}\right\}_{r \geq 0}$, by the geometric spheres $\Sigma_{r}^{h}$ of radius $r<s$. Fix this foliation and let $W$ be a Killing vector field in $\mathbb{R}^{n+1}$. Then $W$ at each point is expressed, modulo an orthogonal transformation of $\mathbb{R}^{n+1}$, as

$$
W(x)=x A
$$

where $x=\left(x_{0}, \ldots, x_{n}\right) \in \mathbb{R}^{n+1}$ and $A$ is a $(n+1) \times(n+1)$ matrix as it is in EQ. 2.8. The flow at each point is conjugate to a diagonal matrix

$$
\operatorname{Rot}\left(a_{1} t, \ldots, a_{k} t\right)
$$

where $\operatorname{Rot}\left(a_{1} t, \ldots, a_{k} t\right)$ is given by EQ. 2.9 with the additional condition that $a_{1}<s$. This condition guarantees that $\|W\|<1$. The flow of such a vector field is as

$$
\varphi(t, x)=x \operatorname{Rot}\left(a_{1} t, \ldots, a_{k} t\right)
$$

Here we have a Randers space $\left(D_{s}, F\right)$, where $F$ is the associated Randers metric to $\left(D_{s}, h ; W\right)$.

4 Observe that in the case that $n+1$ is even there is no geodesics of $h$ invariant under this flow. For instance, consider a group of h-geodesics $\alpha_{i}$ and the corresponding F-geodesics $\gamma_{i}$ (see Lemma 2.2.14), for $i=1, \ldots, n+1$, as following:

$$
\left\{\begin{array}{lll}
\alpha_{1}=(1,0, \ldots, 0) t & ; & \gamma_{1}(t)=t\left(\cos a_{1} t, \sin a_{1} t, 0, \ldots, 0\right) \\
\alpha_{2}=(0,1,0, \ldots, 0) t & ; & \gamma_{2}(t)=t\left(-\sin a_{1} t, \cos a_{1} t, 0, \ldots, 0\right) \\
\vdots & \vdots \\
\alpha_{n}=(0, \ldots, 0,1,0) t & ; & \gamma_{n}(t)=t\left(0, \ldots, 0, \cos a_{k} t, \sin a_{k} t\right) \\
\alpha_{n+1}=(0, \ldots, 0,1) t & ; & \gamma_{n+1}(t)=t\left(0, \ldots, 0,-\sin a_{k} t, \cos a_{k} t\right) .
\end{array}\right.
$$

Here $\alpha_{i}$ coincides with the $x_{i}$-axis and $\gamma_{i}$ is the spiral in the $x_{i} x_{i+1}$ (or $\left.x_{i-1} x_{i}\right)$-plane.

As a special case consider $D_{S} \subset \mathbb{R}^{4}$ and let $a_{1}=\frac{1}{3}$. That is the following geodesics for which $\gamma_{i}$ are drawn for $t \in[0,1000 \pi]$ and $t \in[-1000 \pi, 1000 \pi]$

$$
\begin{cases}\alpha_{1}=(1,0,0,0) t \quad & \gamma_{1}(t)=t\left(\cos \frac{t}{3}, \sin \frac{t}{3}, 0,0\right) \\ \alpha_{2}=(0,1,0,0) t \quad ; \quad \gamma_{2}(t)=t\left(-\sin \frac{t}{3}, \cos \frac{t}{3}, 0,0\right) .\end{cases}
$$

As an interesting fact notice that the geodesics $\alpha_{i}$ intersect just once while $\gamma_{i}$ infinite many times. Here in Picture 2.16, $\gamma_{1}$ is shown with blue and $\gamma_{2}$ with red, where $t \in[0,10 \pi]$ in the left hand picture and $t \in[0,100 \pi]$ in the right hand picture. In the left hand picture of 2.17, $\gamma_{1}$ is shown with blue color and $\gamma_{2}$ with red one where $t \in[0,100 \pi]$. In the right hand picture we have $\gamma_{1}$ for $t \in[-10 \pi, 10 \pi]$. Finally in Picture 2.18, we have $\gamma_{1}$ for $t \in[-100 \pi, 100 \pi]$ and for $t \in[-1000 \pi, 1000 \pi]$.

$\uparrow$ For the case in which $n+1$ is odd, the only h-geodesics invariant under the flow $\varphi$ are 

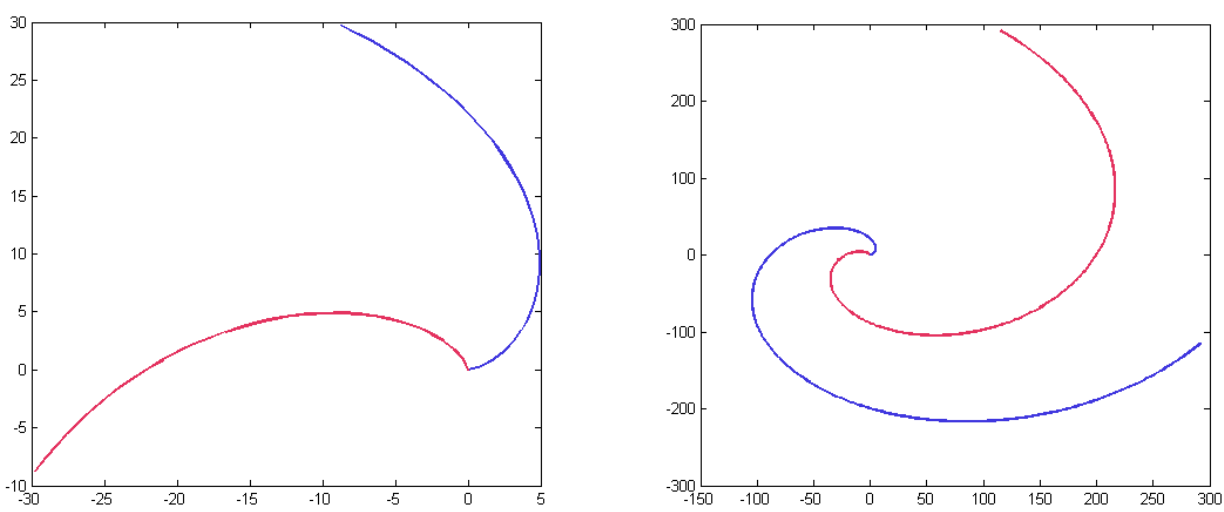

Figure 2.16
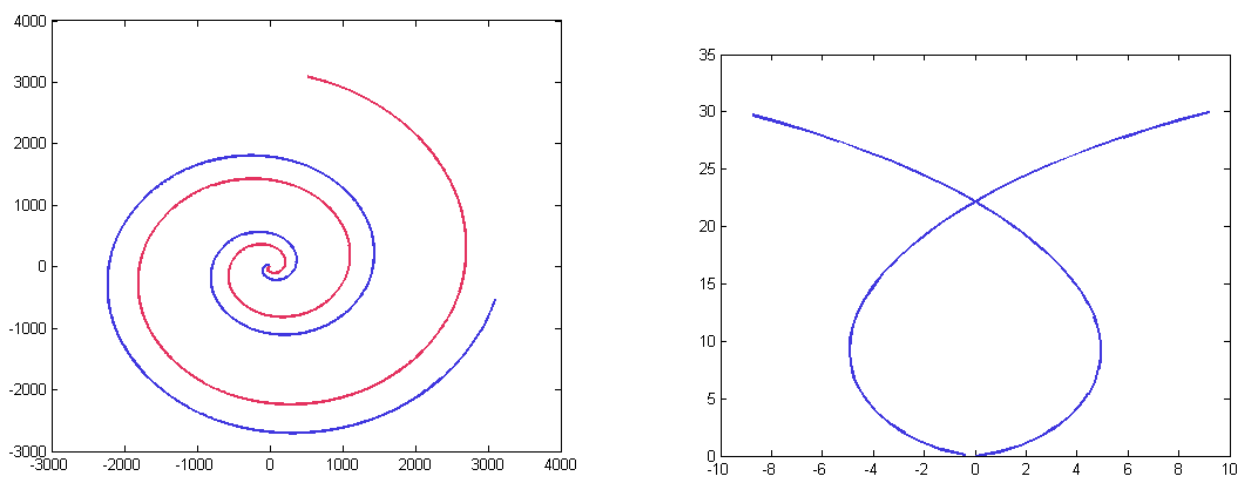

Figure 2.17
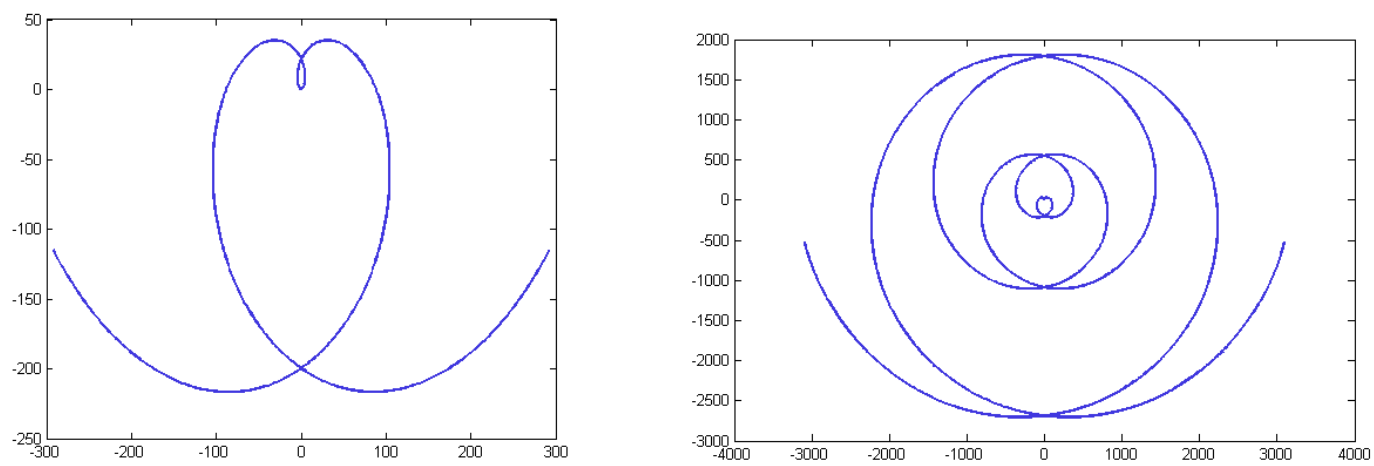

Figure 2.18

reparametrizations of

$$
\alpha_{n+1}(t)=(0, \ldots, 0,1) t
$$

Indeed the associated F-geodesic is $\gamma_{n+1}(t)=\alpha_{n+1}(t) \operatorname{Rot}\left(a_{1} t, \ldots, a_{k} t\right)=\alpha_{n+1}$.

As an interesting case, when $n+1$ is odd, consider the h-geodesics $\alpha(t)$ in which the $(n+1)$-th component and at least one of the $i$-th components, for $i=1, \ldots, n$, are non-zero. Then the corresponding F-geodesic is the hurricane curve

$$
\gamma(t)=\alpha(t) \operatorname{Rot}\left(a_{1} t, \ldots, a_{k} t\right)
$$


As two special cases consider $D_{s} \subset \mathbb{R}^{3}$ with $a=\frac{1}{2}$ with the following $h$ and $F$ geodesics.

$$
\begin{cases}\alpha_{1}=\left(\frac{\sqrt{2}}{2}, 0, \frac{\sqrt{2}}{2}\right) t \quad ; \quad \gamma_{1}(t)=\frac{\sqrt{2}}{2} t\left(\cos \frac{t}{2}, \sin \frac{t}{2}, 1\right), \\ \alpha_{2}=(1,0, t) \quad ; \quad \gamma_{2}(t)=\left(\cos \frac{t}{2}, \sin \frac{t}{2}, t\right) .\end{cases}
$$

See Picture 2.19 where, from the left hand, in the first picture we plot $\gamma_{1}$ for $t \in[0,1000 \pi]$ and in the second one for $t \in[-1000 \pi, 1000 \pi]$. In the right hand picture we have $\gamma_{2}$ for $t \in[0,1000 \pi]$.
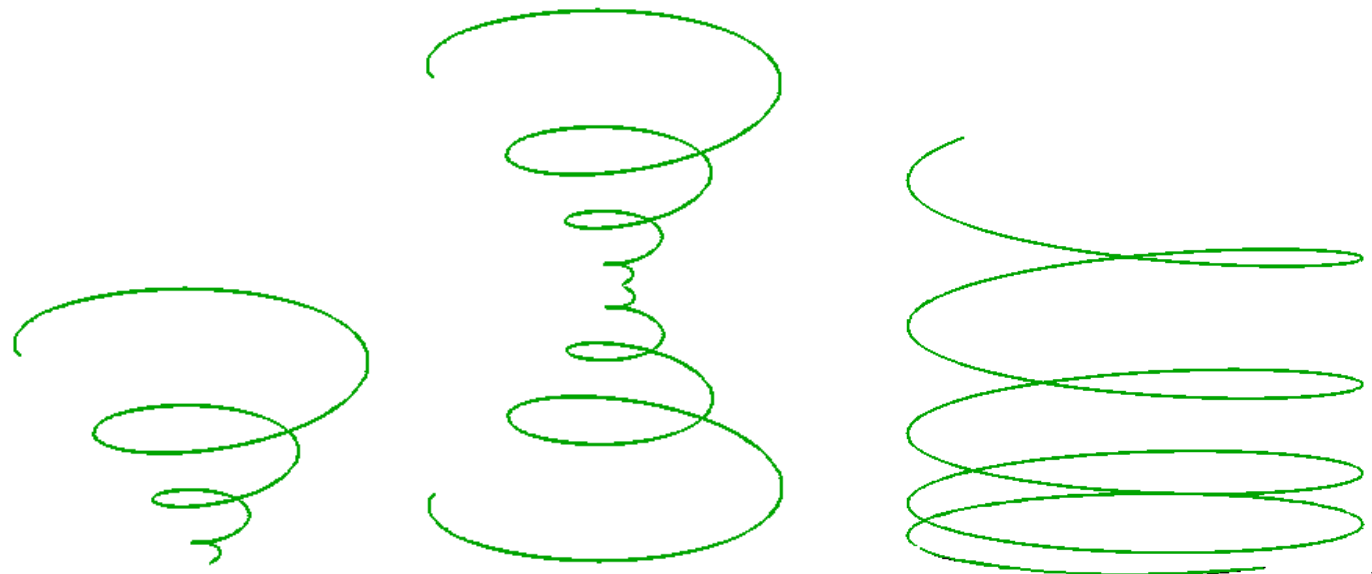

Figure 2.19

To sum up, in these Randers spaces each geodesic is a reparametrization of the following curves

1. If $n+1$ is even, $\gamma(t)$ is a spiral, i.e. $\gamma(t)=\sum_{i=1}^{n+1} r_{i} \gamma_{i}(t)$.

2. If $n+1$ is odd, $\gamma(t)$ is one of the following curves (up to a reparametrization);

- $\gamma(t)$ is a spiral as item (1),

$-\gamma(t)=(0, \ldots, 0, r) t$ whose trace coincides with the $x_{n+1}$ axis where $r \in \mathbb{R} \backslash 0$,

$-\gamma(t)=\sum_{i=1}^{n+1} r_{i} \gamma_{i}(t)+(0, \ldots, 0, r) t$,

where $\gamma_{i}$ is as EQ. 2.12, $r_{i} \in \mathbb{R}$ such that all are not zero, and $r \in \mathbb{R} \backslash 0$. Indeed the wind makes a rotation around the origin for the case of $n+1$ even and a rotation along the $x_{n+1}$-axis for the case of $n+1$ odd.

Let us calculate some of the geometric objects such as the gradient and the Hessian. Note that in this example we have a foliation $\mathcal{F}=\left\{\Sigma_{r}^{h}\right\}_{0 \leq<s}$, by the spheres $\Sigma_{r}^{h}$ of radius $r$ such that $W$ is tangent to the leaves. We first calculate $F(\nabla f)$. By Lemma 2.2.10 we have

$$
F(\nabla f)=\|\bar{\nabla} f\|
$$

Let's calculate $\|\bar{\nabla} f\|$ then. $\bar{\nabla} f=2 x$ and so $F^{2}(\nabla f)=\|\bar{\nabla} f\|^{2}=4 f$. As a result $f$ is $F$-transnormal with $\mathfrak{b}: \mathbb{R} \longrightarrow \mathbb{R}$ as $\mathfrak{b}(t)=4$ t. Observe that this is often so tedious to calculate $\nabla f$, although in this special case in which $W$ is tangent to the leaves by Lemma 2.2.10 we have

$$
\nabla f(x)=2 x+2 \sqrt{f} \frac{x}{\|x\|} A .
$$


Also note that at the critical point 0 , according to Definition 1.3.36, one has Hessf $(0)=2 I_{n+1}$, here by $I_{n+1}$ we mean the identical $(n+1) \times(n+1)$ matrix. Moreover, according to Lemma 1.3.41, in the regular points, we have

$$
\operatorname{Hessf}(\nabla f, \nabla f)=8 f=2 \mathfrak{b} .
$$

In the special case of $D_{s} \subset \mathbb{R}^{2}$, Picture 2.20 shows the foliation and an arbitrary geodesic in the Randers space $\left(D_{s}, F\right)$ for $t \in[0,100 \pi]$ in the left hand picture and for $t \in[-50 \pi, 50 \pi]$ in the right hand picture. As it also can be seen in this picture unlike the original space $\left(D_{s}, h\right)$, in the associated space $\left(D_{s}, F\right)$ a geodesic may intersects itself infinite many times. Although in some cases as that one in Picture 2.19, one geodesic never intersects itself. Consider the geodesics

$$
\gamma(t)=\varphi(t, \alpha(t))=\alpha(t) \operatorname{Rot}\left(a_{1} t, \ldots, a_{k} t\right)
$$

where $\alpha(t)=\frac{p}{\|p\|} t$, for $p \in \Sigma_{r}^{h}$, and $\varphi$ is the flow of $W$. Then

$$
\gamma^{\prime}(t)=\alpha^{\prime}(t) \operatorname{Rot}\left(a_{1} t, \ldots, a_{k} t\right)+\alpha(t) \operatorname{Rot}^{\prime}\left(a_{1} t, \ldots, a_{k} t\right)
$$

Observe that from $\frac{d \varphi}{d t}(t, x)=W(\varphi(t, x))$ one gets

$$
\begin{aligned}
\frac{d \varphi}{d t}(t, \alpha(t))=W(\varphi(t, \alpha(t))) & =\varphi(t, \alpha(t)) A=\alpha(t) \operatorname{Rot}\left(a_{1} t, \ldots, a_{k} t\right) A \\
& =\alpha(t) \operatorname{Rot}^{\prime}\left(a_{1} t, \ldots, a_{k} t\right),
\end{aligned}
$$

from which one has

$$
\gamma^{\prime}(t)=\alpha^{\prime}(t) \operatorname{Rot}\left(a_{1} t, \ldots, a_{k} t\right)+W(\varphi(t, \alpha(t))) .
$$

As a result from EQ. 2.13 and Corollary 2.2.7 one can say that the geodesics $\gamma$ are orthogonal to each leaf. By a similar argument it can be proved that the F-geodesics $\beta(t)$ that are associated to the $h$-geodesics $-\alpha(t)$ are orthogonal to each leaf. Also again from EQ. 2.13, we have that any geodesic which is orthogonal to a leaf, it coincides with $\gamma$ or $\beta$, up to a reparametrization. Because we have that if a unit speed geodesic $\gamma(\beta)$ is orthogonal to a leaf at a point $p$, then $\gamma^{\prime}-W\left(\beta^{\prime}-W\right)$ belongs to the horizontal space, with respect to $h$, at $p$. So $\gamma^{\prime}-W=\frac{p}{\|p\|}\left(\beta^{\prime}-W=-\frac{p}{\|p\|}\right)$.

Observe that as a consequence from above calculations and discussions we have that

$$
\mathcal{F}=\left\{\sum_{r}^{h}\right\}_{0 \leq r<s}
$$

is a SFF. See picture 2.20. We study this example again in Chapter 3.

Example 2.4.8. Consider the open disk $D=\left\{(x, y) \in \mathbb{R}^{2} \mid x^{2}+y^{2}<1\right\}$ as a subset of $\mathbb{R}^{2}$. Put the Killing vector field

$$
W(x, y)=(x, y)\left(\begin{array}{cc}
0 & 1 \\
-1 & 0
\end{array}\right)
$$

on it whose flow is as

$$
\varphi(t,(x, y))=(x, y)\left(\begin{array}{cc}
\cos t & \sin t \\
-\sin t & \cos t
\end{array}\right) .
$$

Let us study some special geodesics in the Riemannian space $(D, h)$ and its associated Randers space $(D, F)$. In fact, for $i=1,2$, we consider two geodesics $\alpha_{i}$ which are the same geodesics in $(D, h)$ and 

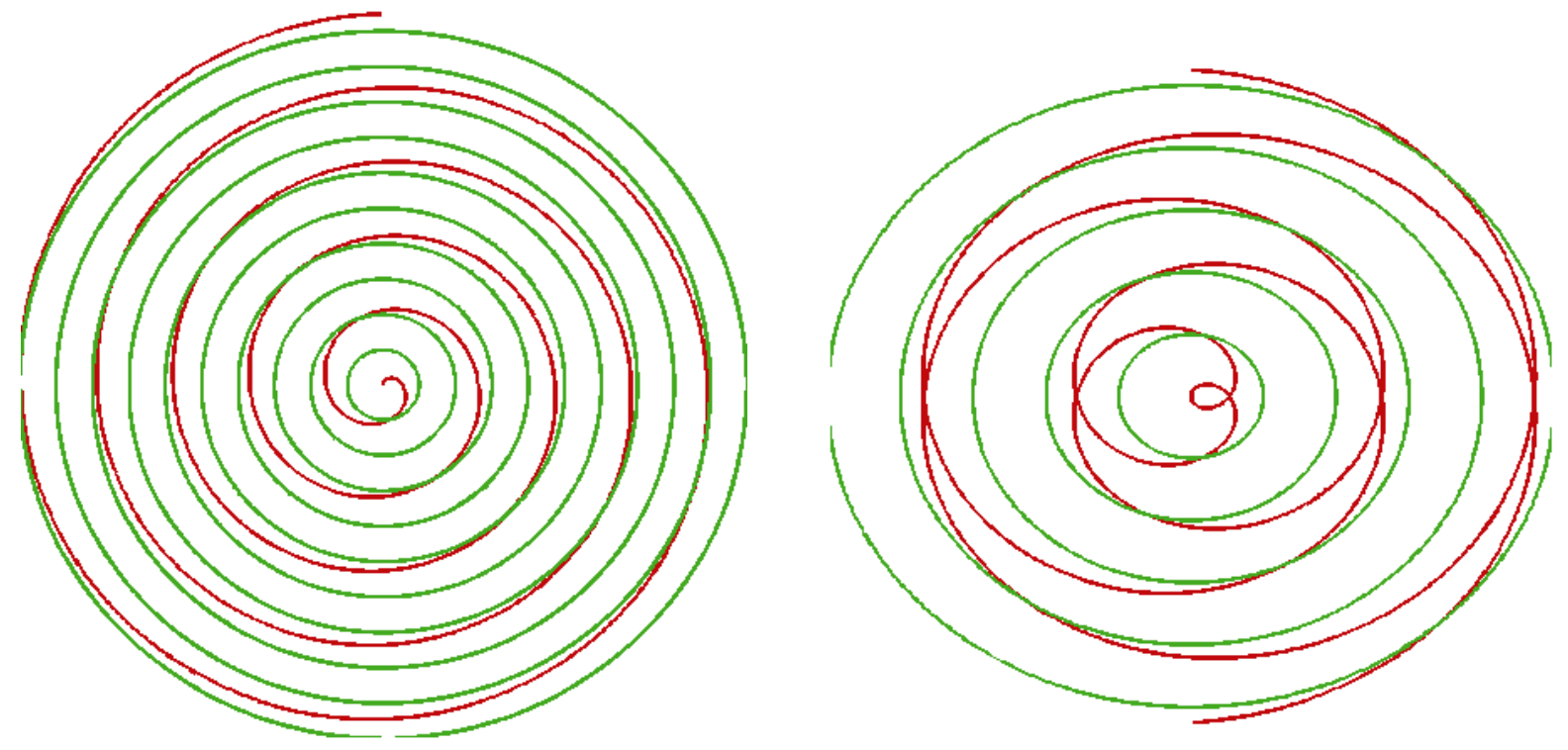

Figure 2.20

then the associated Randers geodesics $\gamma_{i}=\varphi\left(t, \alpha_{i}(t)\right)$ which are two distinct geodesics in $(D, F)$.

case 1. Consider

$$
\begin{cases}\alpha_{1}(t)=(t, 0) & ; \quad \gamma_{1}(t)=t(\cos t, \sin t) \\ \alpha_{2}(t)=\alpha_{1}(-t) \quad ; \quad \gamma_{2}(t)=-\gamma_{1}(t)\end{cases}
$$

Note that $\gamma_{1}$ and $\gamma_{2}$ meet exactly once. See the left hand picture in 2.21.

case 2. Consider

$$
\begin{cases}\alpha_{1}(t)=\frac{t}{2}(\sqrt{2}, \sqrt{2}) & ; \quad \gamma_{1}(t)=\frac{\sqrt{2} t}{2}(\cos t-\sin t, \sin t+\cos t) \\ \alpha_{2}(t)=\alpha_{1}(-t) & ; \quad \gamma_{2}(t)=-\gamma_{1}(t) .\end{cases}
$$

Then $\gamma_{1}$ and $\gamma_{2}$ meet exactly once. See the right hand picture in 2.21.
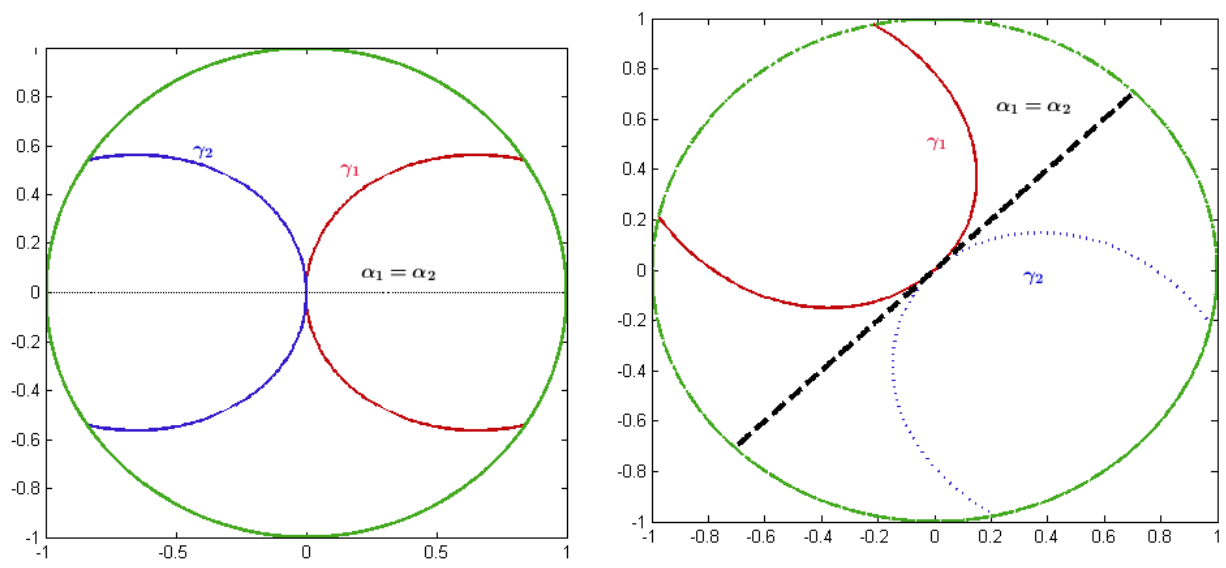

Figure 2.21

case 3. Consider

$$
\begin{cases}\alpha_{1}(t)=\frac{t}{2}(\sqrt{2}, \sqrt{2})+(1,1) & ; \gamma_{1}(t)=\frac{\sqrt{2} t}{2}(\cos t-\sin t, \sin t+\cos t)+(\cos t-\sin t, \sin t+\cos t) \\ \alpha_{2}(t)=\alpha_{1}(-t) & ; \quad \gamma_{2}(t)=-\gamma_{1}(t) .\end{cases}
$$


Then $\gamma_{1}$ and $\gamma_{2}$ meet exactly once. See the left hand picture in 2.22.

case 4. Consider

$$
\begin{cases}\alpha_{1}(t)=\frac{t}{2}(\sqrt{2}, \sqrt{2})+\left(\frac{1}{2}, \frac{1}{2}\right) & ; \gamma_{1}(t)=\frac{\sqrt{2} t}{2}(\cos t-\sin t, \sin t+\cos t)+\frac{1}{2}(\cos t-\sin t, \sin t+\cos t) \\ \alpha_{2}(t)=\alpha_{1}(-t) & ; \gamma_{2}(t)=-\gamma_{1}(t) .\end{cases}
$$

Then $\gamma_{1}$ and $\gamma_{2}$ meet twice. See the right hand picture in 2.22.
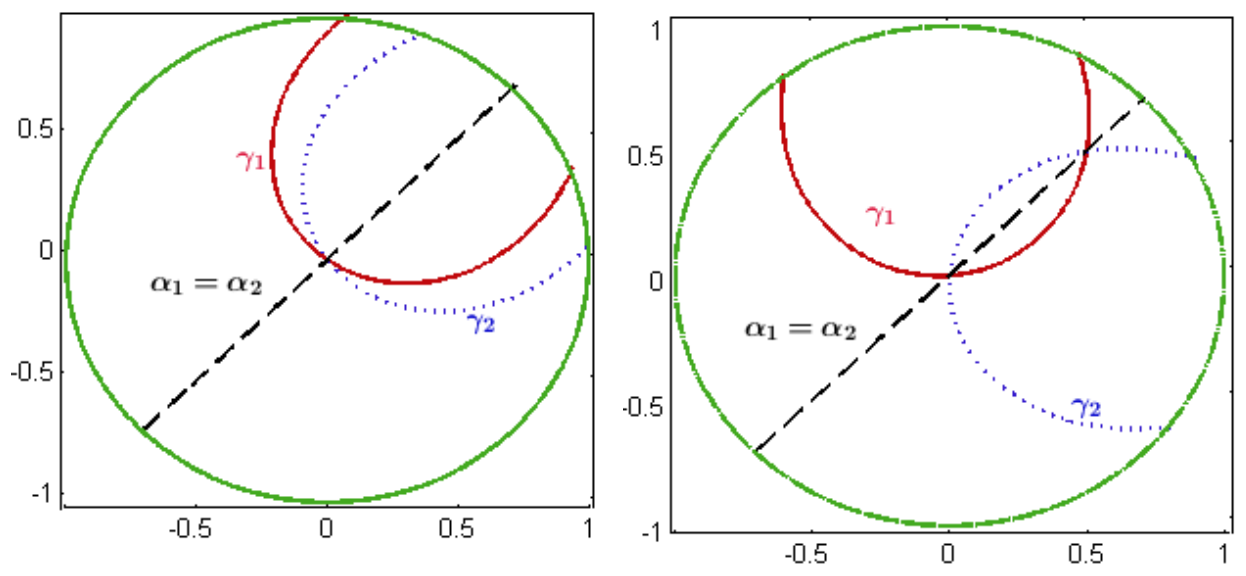

Figure 2.22

case 5. Consider

$$
\begin{cases}\alpha_{1}(t)=(t, 0)+\left(0, \frac{1}{2}\right) & ; \quad \gamma_{1}(t)=t(\cos t, \sin t)+\frac{1}{2}(-\sin t, \cos t) \\ \alpha_{2}(t)=\alpha_{1}(-t) & ; \quad \gamma_{2}(t)=-\gamma_{1}(t) .\end{cases}
$$

Then $\gamma_{1}$ and $\gamma_{2}$ meet exactly once. See the left hand picture in 2.23.

case 6. Consider

$$
\begin{cases}\alpha_{1}(t)=t\left(\frac{\sqrt{2}}{2}, \frac{\sqrt{2}}{2}\right)+\left(\frac{1}{2}, 0\right) & ; \gamma_{1}(t)=\frac{\sqrt{2} t}{2}(\cos t-\sin t, \sin t+\cos t)+\frac{1}{2}(\cos t, \sin t) \\ \alpha_{2}(t)=\alpha_{1}(-t) & ; \gamma_{2}(t)=-\gamma_{1}(t) .\end{cases}
$$

Then $\gamma_{1}$ and $\gamma_{2}$ meet twice. See the right hand picture in 2.23.
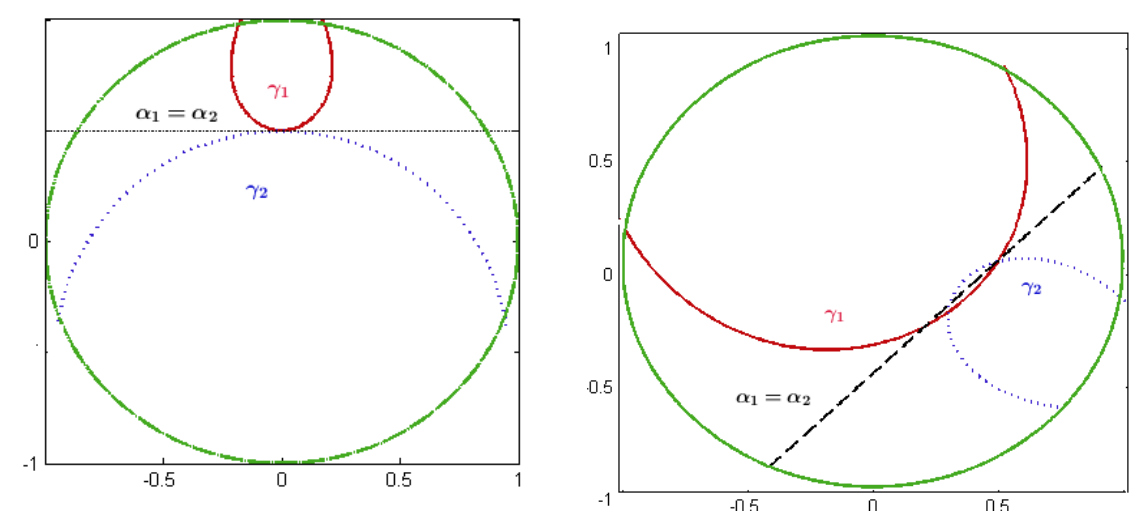

Figure 2.23 
case 7. Consider

$$
\begin{cases}\alpha_{1}(t)=t\left(\frac{\sqrt{2}}{2}, \frac{\sqrt{2}}{2}\right)+\left(\frac{2}{3}, 0\right) & ; \gamma_{1}(t)=\frac{\sqrt{2} t}{2}(\cos t-\sin t, \sin t+\cos t)+\frac{2}{3}(\cos t, \sin t) \\ \alpha_{2}(t)=\alpha_{1}(-t) & ; \gamma_{2}(t)=-\gamma_{1}(t) .\end{cases}
$$

Here $\gamma_{1}$ and $\gamma_{2}$ meet twice. See the picture 2.24.

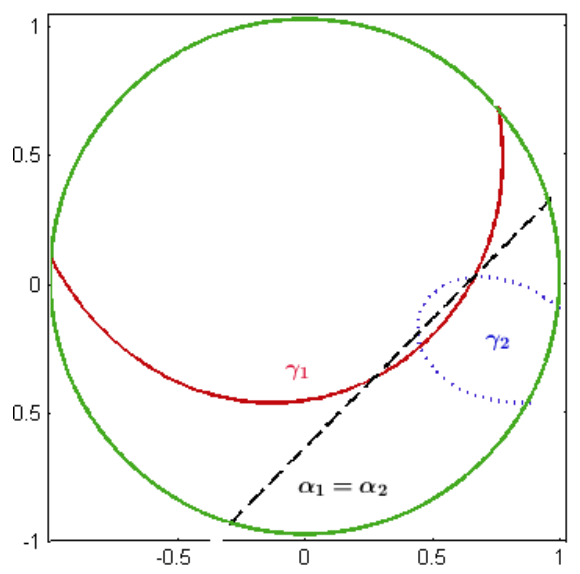

Figure 2.24 
RANDERS SPACES 


\section{Chapter 3}

\section{Some results on Finsler foliations}

\subsection{Introduction}

In this Chapter we show that Wang's Theorem (see Introduction) is not valid in the Finsler case as it is in the Riemannian case. Indeed in the Finsler case we are faced with new phenomena that will be given later in details. One of the main techniques is to use the gradient of $f$. Indeed, a Finsler metric $F$ on $M$ allows one to construct canonically a Riemannian metric $g_{\nabla f}$ on the regular part of the same manifold $M$. Using this metric, it can be checked which properties are inherited to the Finsler space from the Riemannian space. Then, it can be verified which hypothesis are needed in order to prove the results. Moreover, a real function $\mathfrak{b}$ appeared in the definition of the transnormal function allows one to get more information about the foliation. Some of these properties are useful to prove some of the results.

It is natural for one to ask the following three questions in the Finsler case.

Question 3.1.1. Given a Finsler manifold $(M, F)$, are the (regular) level sets of a F-transnormal function $f: M \longrightarrow \mathbb{R}$ parallel? If yes, is the distance between $f^{-1}(c)$ and $f^{-1}(d)$ described by $\int_{c}^{d} \frac{1}{\sqrt{\mathfrak{b}(s)}} d s$ as it was in the Riemannian case?

Question 3.1.2. Under which conditions the level sets of a transnormal function are equidistant?

Question 3.1.3. There are some conditions under which the level sets of a transnormal function on a Finsler manifold are level sets of singular Riemannian foliation for some Riemannian metric?

We first state Proposition 3.2.1 that answers Question 3.1.1 in some direction. Then with an example it will be shown that the answer of this question in the general case is not positive. Next in Theorem 3.3.1 we provide the conditions under which Question 3.1.2 is satisfied. Finally after providing some other related results we will answer Question 3.1.3 positively and provide the necessary conditions.

Through out this Chapter we assume that $(M, F)$ is a connected, forward complete, smooth Finsler manifold and $f: M \longrightarrow \mathbb{R}$ is a smooth function.

\subsection{Answering Question 3.1.1}

As it was mentioned before we mention a theorem which says that the answer of Question 3.1.1 is affirmative in just one direction. In Example 3.2.2 it is shown that one side of this question must be answered negatively. 


\section{Proposition and illustrating example}

Here we state and prove a proposition that is an improvement of Lemma 4.2 of [HYS16]. We also provided an alternative proof for it.

Proposition 3.2.1. Let $f: M \longrightarrow \mathbb{R}$ be a F-transnormal function with $f(M)=[a, b]$. If $c<d \in$ $f(M)$ then

For every $q \in f^{-1}(d)$,

$$
d_{F}\left(f^{-1}(c), q\right)=d_{F}\left(f^{-1}(c), f^{-1}(d)\right)=\int_{c}^{d} \frac{d s}{\sqrt{\mathfrak{b}(s)}}=L(\alpha)
$$

where $\alpha$ is a reparametrization of (the extension of) the integral curve of $\nabla f$.

(). In particular $f^{-1}(c) \| f^{-1}(d)$.

Let's before proceeding with the proof of this proposition, first see an example that illustrates this proposition and shows that one direction of Question 3.1.1 should be answered negatively.

Example 3.2.2. Let us recall Definition 1.4.15 and Example 2.3.1. Let $A=\{(0,0)\}:=0$. We have $\rho_{+}: \mathbb{R}^{2} \longrightarrow \mathbb{R} \geq 0, p \mapsto d_{F}(0, p)$ and it is smooth on $\mathbb{R}^{2} \backslash 0$. Note that by Lemma 1.4.18 this map is F-transnormal and according to Proposition 3.2.1 we must have the parallelism in some direction. Let's study this example more. First note that as we have a Minkowski space the minimizing geodesics joining 0 to $p$ is the straight line $\gamma(t)=p t$. So

$$
d_{F}(0, p)=\int_{0}^{1} F\left(\gamma^{\prime}\right) d t=F(p)
$$

As a consequence,

$\rho_{+}^{-1}(r)=\left\{p \in \mathbb{R}^{2} \mid d_{F}(0, p)=r\right\}=\left\{p \in \mathbb{R}^{2} \mid F(p)=r\right\}=\left\{p \in \mathbb{R}^{2} \mid p_{1}^{2}+\left(p_{2}-\frac{r}{2}\right)^{2}=r^{2}\right\}=\Sigma_{r}^{F}=\Sigma_{r}^{h}+r W$.

So we have a foliation of $\mathbb{R}^{2}$ as $\mathcal{F}=\left\{\rho_{+}^{-1}(r)\right\}_{r \geq 0}$. Since by Lemma 1.4.18, $\rho_{+}$is F-transnormal with $\mathfrak{b}=1$, using Proposition 3.2.1, for $0 \leq c_{0}<c_{1}$, we have $\rho_{+}^{-1}\left(c_{0}\right) \| \rho_{+}^{-1}\left(c_{1}\right)$ with

$$
d_{F}\left(\rho_{+}^{-1}\left(c_{0}\right), \rho_{+}^{-1}\left(c_{1}\right)\right)=\int_{c_{0}}^{c_{1}} d s=c_{1}-c_{0}
$$

Observe that in Definition 1.4 .14 if one lets $P=(0,0)$ then we have $C_{r}^{+}(0)=\rho_{+}^{-1}(r)$. Indeed for

$$
r_{0}<r_{1}, \quad C_{r_{0}}^{+}(0) \| C_{r_{1}}^{+}(0)
$$

with the distance $r_{1}-r_{0}$.

By an analogue calculation we have $\rho_{-}: \mathbb{R}^{2} \longrightarrow \mathbb{R} \geq 0, p \mapsto-d_{F}(p, 0)$ and

$$
\rho_{-}^{-1}(s)=\left\{p \in \mathbb{R}^{2} \mid-d_{F}(p, 0)=s\right\} .
$$

The minimizing geodesic joining $p$ to 0 is the straight line $\gamma(t)=-p t$. So

$$
d_{F}(0, p)=\int_{-1}^{0} F\left(\gamma^{\prime}\right) d t=F(-p)
$$



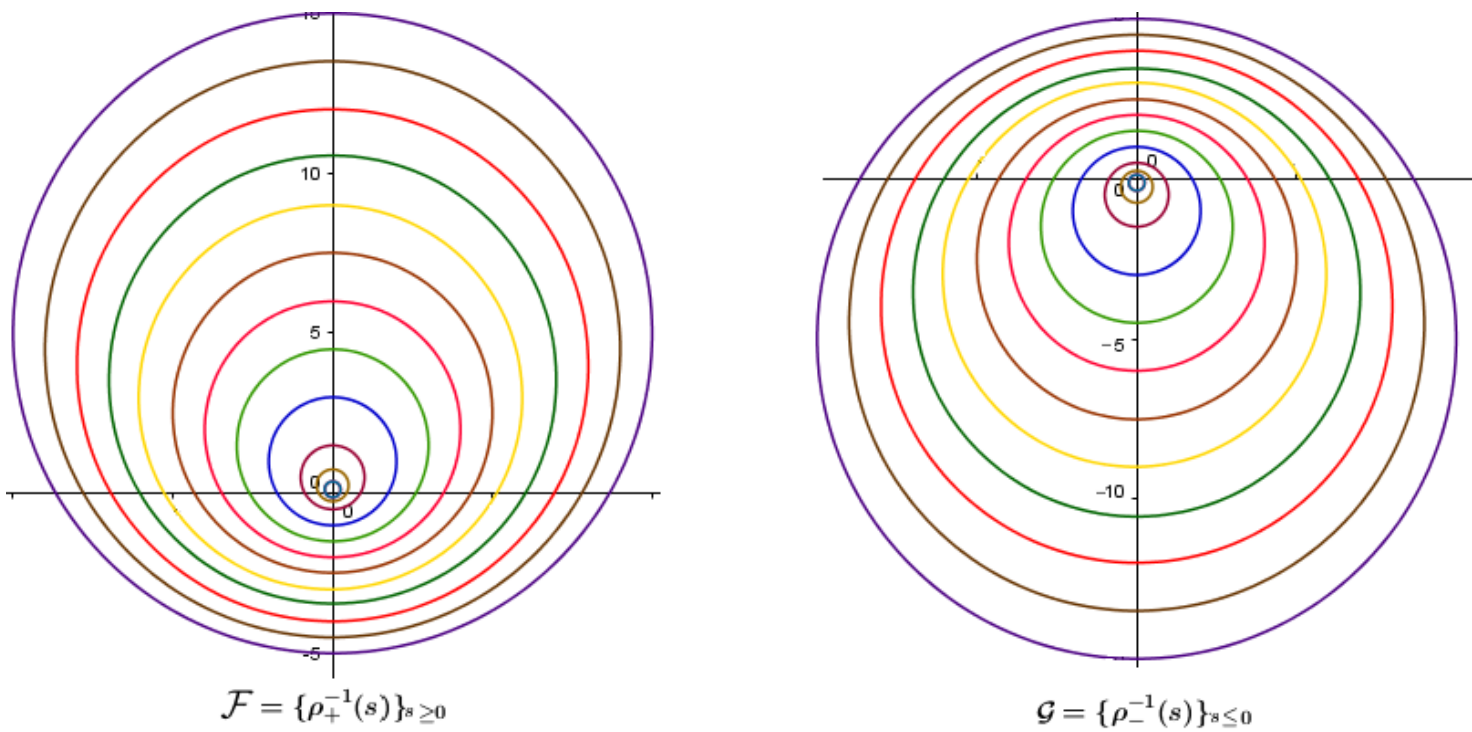

Figure 3.1: Two foliations of $\mathbb{R}^{2}$

and

$$
\rho_{-}^{-1}(s)=\left\{p \in \mathbb{R}^{2} \mid-F(-p)=s\right\}=\left\{p \in \mathbb{R}^{2} \mid p_{1}^{2}+\left(p_{2}-\frac{s}{2}\right)^{2}=s^{2}\right\}=\Sigma_{-s}^{h}+s W .
$$

So we have a foliation of $\mathbb{R}^{2}$ as $\mathcal{G}=\left\{\rho_{-}^{-1}(s)\right\}_{s \leq 0}$ and by Proposition 3.2.1 for $d_{1}<d_{0}<0$, $\rho_{-}^{-1}\left(d_{1}\right) \| \rho_{-}^{-1}\left(d_{0}\right)$ with

$$
d_{F}\left(\rho_{-}^{-1}\left(d_{1}\right), \rho_{-}^{-1}\left(d_{0}\right)\right)=\int_{d_{1}}^{d_{0}} d s=d_{0}-d_{1}
$$

and $C_{-s}^{-}(0)=\rho_{-}^{-1}(s)$. Indeed for $s_{1}<s_{0} \leq 0, C_{-s_{1}}^{-}(0) \| C_{-s_{0}}^{-}(0)$ with the distance $s_{0}-s_{1}$.

In this example we have two foliations for $\mathbb{R}^{2}$ one by the past cylinders and another by the future cylinders that can be seen in Picture 3.1. Observe that these two foliations do not coincide. It is notable that

$$
C_{r_{1}}^{+}(0) \nVdash C_{r_{0}}^{+}(0) \text { and } C_{s_{0}}^{-}(0) \nVdash C_{s_{1}}^{-}(0) \text {. }
$$

The first case is shown in Example 1.4.12 and for the second case, for example consider $C_{\frac{1}{3}}^{-}(0)$ and $C_{\frac{2}{3}}^{-}(0)$. On the one hand, for the point $\left(0, \frac{1}{2}\right) \in C_{\frac{1}{3}}^{-}(0)$, the geodesic $\gamma(t)=\left(0, \frac{1}{2}\right) t$ minimizes the distance from $\left(0, \frac{1}{2}\right)$ to $C_{\frac{2}{3}}^{-}(0)$. So we have that

$$
d_{F}\left(\left(0, \frac{1}{2}\right), C_{\frac{2}{3}}^{-}(0)\right)=\int_{1}^{2} F\left(0, \frac{1}{2}\right)=\frac{1}{3}
$$

While, on the other hand, for point $\left(-\frac{3}{2}, 0\right) \in C_{\frac{1}{3}}^{-}(0)$ the geodesic $\gamma(t)=-(0,3) t$ minimizes the distance from $\left(-\frac{3}{2}, 0\right)$ to $C_{\frac{2}{3}}^{-}(0)$. Hence we have that

$$
d_{F}\left(\left(-\frac{3}{2}, 0\right), C_{\frac{2}{3}}^{-}(0)\right)=\int_{-\frac{1}{2}}^{1} F(-(0,3))=3 .
$$

According to EQs. 3.1 and 3.2 one concludes that

$$
C_{\frac{1}{3}}^{-}(0) \nVdash C_{\frac{2}{3}}^{-}(0) .
$$




\subsubsection{The proof of Proposition 3.2.1}

In this part we prove proposition 3.2.1 through some steps. Firstly, we mention a comment that gives a key about the direction of the gradient vector. Then we restate the expression of proposition.

Comment 3.2.3. Assume that $f: M \longrightarrow \mathbb{R}$ is a smooth function and $\gamma: I \longrightarrow M$ is the integral curve of the gradient, namely $\gamma^{\prime}=\nabla f(\gamma)$. Define $g: I \longrightarrow \mathbb{R}$ with $g(t)=f(\gamma(t))$ and notice that $g$ is an increasing function. Since

$$
g^{\prime}(t)=(f \circ \gamma)^{\prime}(t)=d f(\nabla f)=F^{2}(\nabla f) \geq 0 .
$$

As a consequence $f$ is increasing in the direction of the gradient.

Proposition. Let $f: M \longrightarrow \mathbb{R}$ be a F-transnormal function with $f(M)=[a, b]$. If $c<d \in f(M)$ then

For every $q \in f^{-1}(d)$,

$$
d_{F}\left(f^{-1}(c), q\right)=d_{F}\left(f^{-1}(c), f^{-1}(d)\right)=\int_{c}^{d} \frac{d s}{\sqrt{\mathfrak{b}(s)}}=L(\alpha),
$$

where $\alpha$ is a reparametrization of (the extension of) the integral curve of $\nabla f$.

() In particular $f^{-1}(c) \| f^{-1}(d)$.

Remark 3.2.4. Observe that in this Theorem the values a and $b$ might be even infinity.

As the first step we state and prove a Proposition which is the Theorem in the special case in which there is no critical values in the interval of the image. After that the cases containing the critical values are verifying separately.

Proposition 3.2.5. Suppose that the interval $[c, d] \in f(M)$ does not have any critical value of $f$. Then for every $q \in f^{-1}(d)$

$$
d_{F}\left(f^{-1}(c), q\right)=d_{F}\left(f^{-1}(c), f^{-1}(d)\right)=\int_{c}^{d} \frac{d s}{\sqrt{\mathfrak{b}(s)}}=L(\alpha),
$$

where $\alpha$ is the reparametrization of the integral curve of $\nabla f$ as $\alpha\left(\frac{t}{F(\nabla f)}\right)$.

In particular $f^{-1}(c) \| f^{-1}(d)$.

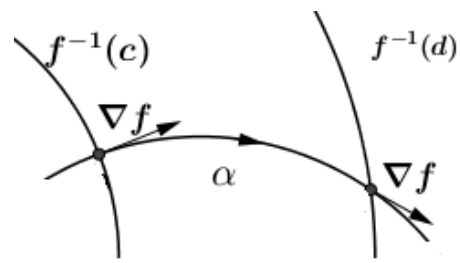

Figure 3.2: Proposition 3.2.5

Proof. First of all, with respect to Proposition 1.3.31, $f$ is a transnormal function with respect to the Riemannian metric $\hat{g}:=g_{\nabla f}$ and

$$
\hat{g}(\hat{\nabla} f, \hat{\nabla} f)=F^{2}(\hat{\nabla} f)=\mathfrak{b} \circ f .
$$


Furthermore given $x \in f^{-1}(c)$, according to the Riemannian case, there exists an integral curve $\gamma$ of $\hat{\nabla} f=\nabla f$ emanating from $x \in f^{-1}(c)$ which is extended to $f^{-1}(d)$ and meets all of the leaves between $f^{-1}(c)$ and $f^{-1}(d)$ orthogonally. Moreover, notice that

$$
t \longrightarrow \gamma\left(\frac{t}{\|\hat{\nabla} f\|}\right)
$$

is a geodesic with respect to $\hat{g}$ and so by Corollary 1.3 .7 it is also a geodesic with respect to $F$.

Now let us prove that $\gamma$ is a minimizing geodesic, up to a reparametrization. Let $x_{0} \in f^{-1}(d)$. If $\beta:\left[0, t_{1}\right] \longrightarrow M$ with $\beta(0) \in f^{-1}(c)$ and $\beta\left(t_{1}\right)=x_{0}$ is a geodesic which minimizes the distance

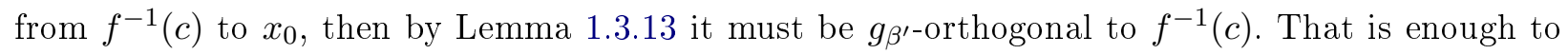
prove that $\beta$ is a reparametrization of the integral curve of $\nabla f$. First notice that $\left.\beta\right|_{\left(0, t_{1}\right)}$ does not intersect $f^{-1}(c)$. Since if on the contrary $\beta\left(t_{0}\right) \in f^{-1}(c)$, for some $t_{0} \in\left(0, t_{1}\right)$, then for $\tilde{\beta}=\left.\beta\right|_{\left[t_{0}, t_{1}\right]}$ we have that $\tilde{\beta}$ joins $f^{-1}(c)$ to $x_{0}$ with $L(\tilde{\beta}) \supsetneqq L(\beta)$ which is absurd (see Picture 3.3). Now as

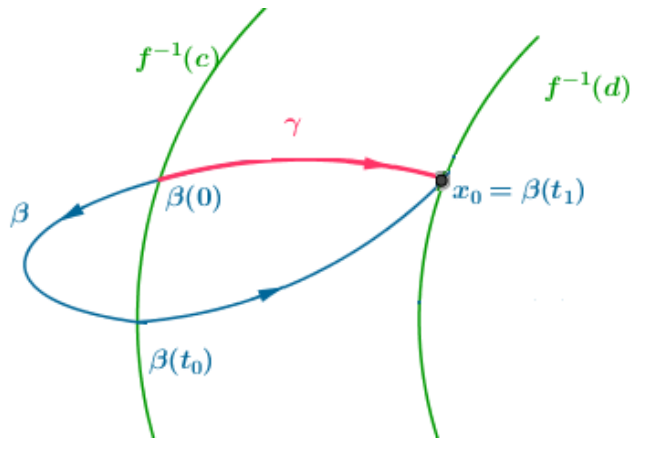

Figure 3.3

$\beta^{\prime}(0) \in \mathfrak{C}_{\beta(0)}\left(f^{-1}(c)\right)$ and $f^{-1}(c)$ has codimension $1, \beta^{\prime}(0)$ is in the direction of $\nabla f$ or on the opposite side of $\nabla f$ with respect to the hyperplane $T_{\gamma(0)} f^{-1}(c)$. The second case implies that $\beta$ must intersect $f^{-1}(c)$ at some time $t_{0} \in\left(0, t_{1}\right)$ which is absurd with respect to above argument. So as a consequence $\beta^{\prime}=\lambda \nabla f$ for some $\lambda>0$. Now as $\gamma$ and $\beta$ are both geodesics emanating from the same point whose velocities are positively proportional to the gradient, by the unicity of the geodesics, they coincide, by a reparametrization.

Finally,

$$
\begin{aligned}
d_{F}\left(f^{-1}(c), x_{0}\right) & =\int_{0}^{l} F\left(\gamma^{\prime}\right) d t=\int_{0}^{l} F(\nabla f(\gamma)) d t=\int_{0}^{l} \hat{F}(\hat{\nabla} f(\gamma)) d t=\int_{0}^{l} \frac{\hat{g}(\hat{\nabla} f, \hat{\nabla} f)}{\hat{F}(\hat{\nabla} f(\gamma))} d t=\int_{0}^{l} \frac{d f(\hat{\nabla} f)}{\sqrt{\mathfrak{b}(f(\gamma))}} d t \\
& =\int_{0}^{l} \frac{(f \circ \gamma)^{\prime}}{\sqrt{\mathfrak{b} \circ f \circ \gamma}} d t=\int_{c}^{d} \frac{d s}{\sqrt{\mathfrak{b}(s)}} .
\end{aligned}
$$

We just proved that $d_{F}\left(f^{-1}(c), x_{0}\right)=\int_{c}^{d} \frac{d s}{\sqrt{\mathfrak{b}(s)}}$. As this integral does not depend on $x_{0} \in f^{-1}(d)$, it can be deduced that for every $q \in f^{-1}(d)$ the same equality holds which closes the proof.

Remark 3.2.6. Observe that as a consequence of Proposition 3.2.5, one can say that for every $x \in f^{-1}(c)$ the reparametrization

$$
t \longrightarrow \gamma\left(\frac{t}{F(\nabla f)}\right)
$$

of the integral curve of $\nabla f$ is the unique minimizing geodesic joining $x$ to $\gamma\left(d_{F}\left(f^{-1}(c), f^{-1}(d)\right)\right) \in$ $f^{-1}(d)$. 
As the second step we write a lemma with the same expression as Lemma 2 given (for the Riemannian case) in [Wan87] whose proof is done in the same way, mutatis mutandis. So we only mention the expression of it.

Lemma 3.2.7. Let $b$ be the only critical value of $f$ in the interval $[c, b]$. Then the improper integral $\int_{c}^{b} \frac{d s}{\sqrt{\mathfrak{b}(s)}}$ converges to $d_{F}\left(f^{-1}(c), f^{-1}(b)\right)$. In other words,

$$
d_{F}\left(x, f^{-1}(b)\right)=\int_{c}^{b} \frac{d s}{\sqrt{\mathfrak{b}(s)}},
$$

for every $x \in f^{-1}(c)$.

In particular, for the case that $f^{-1}(b)$ is a submanifold, $f^{-1}(c) \| f^{-1}(b)$.

Remark 3.2.8. We have the same result for the case in which a is the only critical value of $f$ in the interval $[a, c]$ which means that the improper integral $\int_{a}^{c} \frac{d s}{\sqrt{\mathfrak{b}(s)}}$ converges to $d_{F}\left(f^{-1}(a), f^{-1}(c)\right)$. In other words,

$$
d_{F}\left(x, f^{-1}(c)\right)=\int_{a}^{c} \frac{d s}{\sqrt{\mathfrak{b}(s)}},
$$

for every $x \in f^{-1}(a)$.

In particular, for the case that $f^{-1}(a)$ is a submanifold, $f^{-1}(a) \| f^{-1}(c)$.

As a more general case, from Lemma 3.2.7 and Remark 3.2.8, we have the following lemma.

Lemma 3.2.9. If $a, b$ are the only critical values of $f$ in the interval $[a, b]$, then the improper integral $\int_{a}^{b} \frac{d s}{\sqrt{\mathfrak{b}(s)}}$ converges to $d_{F}\left(f^{-1}(a), f^{-1}(b)\right)$. In other words,

$$
d_{F}\left(x, f^{-1}(b)\right)=\int_{a}^{b} \frac{d s}{\sqrt{\mathfrak{b}(s)}},
$$

for every $x \in f^{-1}(a)$.

In particular, for the case that $f^{-1}(a)$ and $f^{-1}(b)$ are submanifolds, $f^{-1}(a) \| f^{-1}(b)$.

Proof. Choose a regular value $c \in(a, b)$. According to Lemma 3.2.7 and Remark 3.2.8 we have that

$$
d_{F}\left(x, f^{-1}(c)\right)=\int_{a}^{c} \frac{d s}{\sqrt{\mathfrak{b}(s)}}=r
$$

for every $x \in f^{-1}(a)$ and

$$
d_{F}\left(y, f^{-1}(b)\right)=\int_{c}^{b} \frac{d s}{\mathfrak{b}(s)}=s
$$

for every $y \in f^{-1}(c)$. So given $x \in f^{-1}(a)$ there exists a unitary speed geodesic $\gamma$ emanating from $x$ such that

$$
d_{F}\left(x, f^{-1}(c)\right)=L(\gamma)=r .
$$

Also there exists a unitary speed geodesic $\beta$ emanating from $\gamma(r) \in f^{-1}(c)$ such that

$$
d_{F}\left(\gamma(r), f^{-1}(b)\right)=L(\beta)=s
$$


Note that with respect to Lemma 1.3.13, both $\gamma$ and $\beta$ intersect the leaf $f^{-1}(c)$ orthogonally. Hence, in fact, $\beta$ is an extension of $\gamma$ and so $\gamma(r+s)=\beta(s) \in f^{-1}(b)$. So

$$
d_{F}\left(x, f^{-1}(b)\right)=r+s=\int_{a}^{c} \frac{d s}{\sqrt{\mathfrak{b}(s)}}+\int_{c}^{b} \frac{d s}{\sqrt{\mathfrak{b}(s)}}=\int_{a}^{b} \frac{d s}{\sqrt{\mathfrak{b}(s)}}
$$

and as this distance does not depend on $x$ it closes the proof.

Remark 3.2.10. To sum up above results, for the case that $f^{-1}(a)$ and $f^{-1}(b)$ are submanifolds,

$$
f^{-1}(a) \| f^{-1}(c) \text { and } f^{-1}(c) \| f^{-1}(b),
$$

for every $c \in[a, b]$, and each minimizing geodesic from $f^{-1}(a)$ toward $f^{-1}(b)$ meets all of the leaves orthogonally and joins $f^{-1}(a)$ to $f^{-1}(b)$.

Remark 3.2.11. As a result of Proposition 3.2.1, according to Lemma 1.4.27, one can say that for some $r \in \mathbb{R}$ we have

$$
f^{-1}(d) \subset C_{r}^{+}\left(f^{-1}(c)\right) \text { and } f^{-1}(c) \subset C_{r}^{-}\left(f^{-1}(d)\right) .
$$

\subsubsection{Examples}

Example 3.2.12. Let us recall Example 2.4.7. That is for $D_{s} \subset \mathbb{R}^{n+1}$ we have the space $\left(D_{s}, h\right)$ where $h$ is the canonical metric and $f: D_{s} \longrightarrow \mathbb{R}$ with $f(x)=\|x\|^{2}$, that is the square of the distance function. So we have a foliation $\mathcal{F}=\left\{\Sigma_{r}^{h}\right\}_{r \geq 0}$, by the geometrical spheres $\Sigma_{r}^{h}$ of radius $r<s$. Fix this foliation and let

$$
W(x)=x A
$$

where $x=\left(x_{0}, \ldots, x_{n}\right) \in \mathbb{R}^{n+1}$ and $A$ is a $(n+1) \times(n+1)$ matrix of the following form

$$
A=\left\{\begin{array}{lll}
a_{1} J \oplus \ldots \oplus a_{k} J \oplus 0 & \text { if } & n=2 k \\
a_{1} J \oplus \ldots \oplus a_{k} J & \text { if } & n=2 k-1 .
\end{array}\right.
$$

As we discussed in this example, $f$ is a F-transnormal function with $\mathfrak{b}(t)=4 t$. Now using Proposition 3.2.1 one has that for $0<r_{1}<s_{1}, \Sigma_{r_{1}}^{h} \| \Sigma_{s_{1}}^{h}$ with

$$
d_{F}\left(\Sigma_{r_{1}}^{h}, \Sigma_{s_{1}}^{h}\right)=\int_{r_{1}}^{s_{1}} \frac{1}{2 \sqrt{t}} d t=\sqrt{s_{1}}-\sqrt{r_{1}}
$$

Moreover the integral curve of $\nabla f$ is a minimizing geodesic that meets each $\Sigma_{r}^{h}$ orthogonally. As a special case, see the geodesics and leaves in the Randers space associated to $\left(D_{s}, h, W\right)$, where $h$ is the canonical Euclidean metric, $n+1=2$ and

$$
W(x)=x\left(\begin{array}{cc}
0 & \frac{1}{3} \\
-\frac{1}{3} & 0
\end{array}\right)
$$

shown in Picture 3.4 in which two geodesics $\gamma_{1}$ and $\gamma_{2}$ are as:

$$
\left\{\begin{array}{l}
\alpha_{1}(t)=t(1,0) \quad ; \quad \gamma_{1}(t)=t\left(\cos \frac{t}{3}, \sin \frac{t}{3}\right) \\
\alpha_{2}(t)=t(0,1) ; \quad \gamma_{2}(t)=t\left(-\sin \frac{t}{3}, \cos \frac{t}{3}\right) .
\end{array}\right.
$$


Here in the right hand picture we have $\gamma_{1}$ and $\gamma_{2}$ for $t \geq 0$ and in the left hand we have $\gamma_{\text {! for }}$
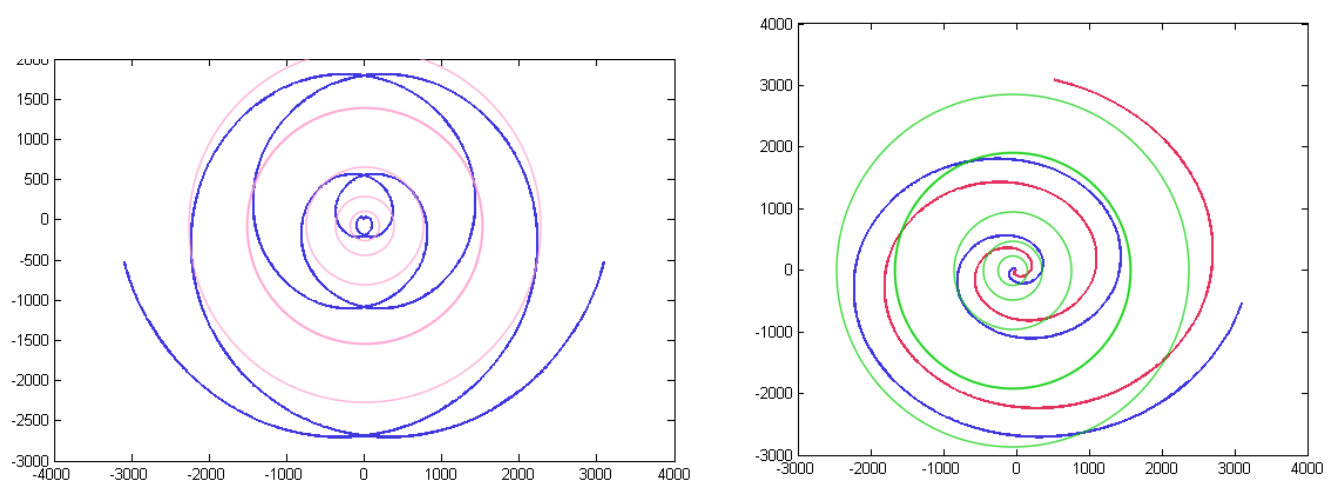

Figure 3.4

$t \in \mathbb{R}$

Example 3.2.13. Consider the Randers metric associated to the data $\left(\mathbb{R}^{2}, h,\left(0, \frac{1}{2}\right)\right)$, where $h$ is the Euclidean canonical metric. Let $f: \mathbb{R}^{2} \longrightarrow \mathbb{R}$ with $f(x, y)=x^{2}$. Then we are faced with a foliation of $\mathbb{R}^{2}$ as the straight lines

$$
\mathcal{F}=\left\{f^{-1}(r)\right\}_{r \geq 0} .
$$

Let's see if $f$ is F-transnormal or not. Observe that according to Lemma 2.2.10, that is enough

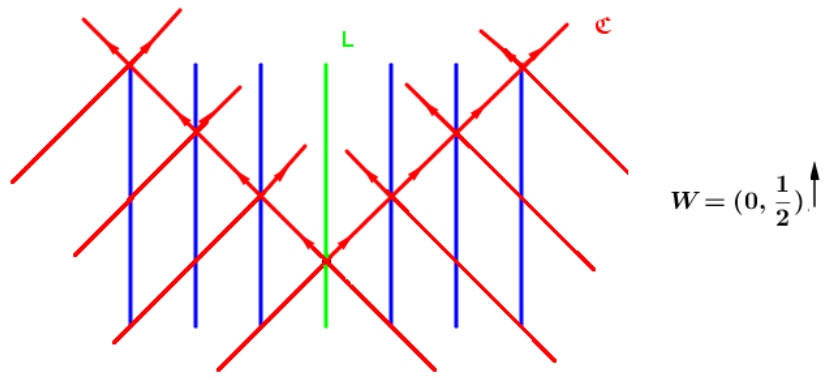

Figure 3.5

to calculate the norm of gradient of $f$ with respect to $h$. So $\bar{\nabla} f=x(2,0)$ and $\|\bar{\nabla} f\|^{2}=4 x^{2}=4 f$. Hence $F^{2}(\nabla f)=4 f$. So with respect to Proposition 3.2.1 we have

$$
f^{-1}(c) \| f^{-1}(d) \text { and } d_{F}\left(f^{-1}(c), f^{-1}(d)\right)=\int_{c}^{d} \frac{d t}{2 \sqrt{t}}=\sqrt{d}-\sqrt{c} .
$$

Notice that in this example each regular leaf is a disconnected submanifold which contains two connected submanifolds in different sides of the singular leaf $\left(f^{-1}(0)=L\right)$. It is not also difficult to show that we are faced with a SFF. Indeed as the h-geodesics $\alpha(t)$ parallel (in the sense of parallel lines in Euclidean space) to $\beta(t)=(0, t)$ are orthogonal to each leaf with respect to $h$, then by Corollary 2.2.7, the F-geodesics

$$
\gamma(t)=\varphi(t, \alpha(t))=\left(0, \frac{1}{2}\right) t+\alpha(t)
$$

where $\varphi(t,$.$) is the flow of W$, are orthogonal to each leaf with respect to $F$. Moreover, again by Corollary 2.2.7, each F-geodesic that is orthogonal to a leaf with respect to $F$ coincides with $\gamma(t)$, 
up to a reparametrization, and so it remains orthogonal to all leaves.

\subsection{Answering question 3.1.2}

In this section we provide the sufficient condition with which the leaves of a partition would be equidistant. In other words we state and prove the following theorem. Afterward some example is provided.

\subsubsection{Theorem and proof}

Theorem 3.3.1. Let $f: M \longrightarrow \mathbb{R}$ be a F-transnormal and analytic function on a compact manifold $M$ with $f(M)=[a, b]$. Suppose that $\mathcal{F}=\left\{f^{-1}(c)\right\}_{c \in[a, b]}$ is a singular foliation, the level sets are connected, $a$ and $b$ are the only critical values and that $f^{-1}(a)$ and $f^{-1}(b)$ are singular leaves. Then there exist $r_{c, a}^{ \pm}, r_{c, b}^{ \pm}>0$ such that

$f^{-1}(c)=C_{r_{c, a}^{ \pm}}^{ \pm}\left(f^{-1}(a)\right)=C_{r_{c, b}^{ \pm}}^{ \pm}\left(f^{-1}(b)\right)$, for every $c \in(a, b)$.

In particular,

() for every $c, d \in[a, b], f^{-1}(c)$ is equidistant to $f^{-1}(d)$, i.e. $f^{-1}(c) \| f^{-1}(d)$ and $f^{-1}(d) \|$ $f^{-1}(c)$.

() $\mathcal{F}$ is a $S F F$.

Proof. From the hypothesis, $f^{-1}(b)$ is a submanifold of codimension greater than 1 . So one is allowed to talk about the past and future cylinders of axis $f^{-1}(b)$ and in particular each is a connected submanifold of codimension 1 . Given $c \in[a, b]$ we divide the proof into the following three lemmas.

Lemma 3.3.2. $f^{-1}(c)=C_{r_{c, b}^{-}}^{-}\left(f^{-1}(b)\right)=C_{r_{c, a}^{+}}^{+}\left(f^{-1}(a)\right)$, where $r_{c, b}^{-}=d_{F}\left(f^{-1}(c), f^{-1}(b)\right)$ and $r_{c, a}^{+}=$ $d_{F}\left(f^{-1}(a), f^{-1}(c)\right)$. In particular, $f^{-1}(b)$ and $f^{-1}(a)$ are embedded submanifolds.

Lemma 3.3.3. $f^{-1}(c)=C_{r_{c, b}^{+}}^{+}\left(f^{-1}(b)\right)$, where $r_{c, b}^{+}=d_{F}\left(f^{-1}(b), f^{-1}(c)\right)$.

Lemma 3.3.4. $f^{-1}(c)=C_{r_{c, a}^{-}}^{-}\left(f^{-1}(a)\right)$, where $r_{c, a}^{-}=d_{F}\left(f^{-1}(c), f^{-1}(a)\right)$.

Proof of Lemma 3.3.2. Firstly, by Theorem 3.2.1, for every $x \in f^{-1}(c)$,

$$
d_{F}\left(x, f^{-1}(b)\right)=\int_{c}^{b} \frac{d s}{\sqrt{\mathfrak{b}(s)}}:=r_{c, b}^{-} .
$$

Secondly, by the definition of the past cylinder, $x \in C_{r_{c, b}^{-}}^{-}\left(f^{-1}(b)\right)$ which means

$$
f^{-1}(c) \subset C_{r_{c, b}^{-}}^{-}\left(f^{-1}(b)\right)
$$

Let us prove the other inclusion. For every $x \in C_{r_{c, b}^{-}}^{-}\left(f^{-1}(b)\right)$, there exists $c_{0} \in[a, b]$ such that $x \in f^{-1}\left(c_{0}\right)$. By Theorem 3.2.1, $f^{-1}\left(c_{0}\right) \subset C_{r_{c, b}^{-}}^{-}\left(f^{-1}(b)\right)$ and also

$$
\int_{c_{0}}^{b} \frac{d s}{\sqrt{\mathfrak{b}(s)}}=d_{F}\left(f^{-1}\left(c_{0}\right), f^{-1}(b)\right)=r_{c, b}^{-}
$$


To summarize we have $\int_{c_{0}}^{b} \frac{d s}{\sqrt{\mathfrak{b}(s)}}=\int_{c}^{b} \frac{d s}{\sqrt{\mathfrak{b}(s)}}$ and as $b$ is the maximum value it implies that $c_{0}=c$. It means

$$
C_{r_{c, b}^{-}}^{-}\left(f^{-1}(b)\right) \subset f^{-1}(c) \text { and so } C_{r_{c, b}^{-}}^{-}\left(f^{-1}(b)\right)=f^{-1}(c) .
$$

Note that from the relation $C_{r_{c, b}^{-}}^{-}\left(f^{-1}(b)\right)=f^{-1}(c)$.

By following the same argument as above one can provide the proof of

$$
f^{-1}(c)=C_{r_{c, a}^{+}}^{+}\left(f^{-1}(a)\right)
$$

Proof of Lemma 3.3.3. To begin with, we state and prove a very useful lemma that we use it several times during the proof of Theorem.

Statement 3.3.5. If $u, v \in \mathfrak{C}^{1}\left(f^{-1}(b)\right)$ then

$$
f(\exp t v)=f(\exp t u)
$$

for every $t>0$.

Proof. Given $u, v \in \mathfrak{C}^{1}\left(f^{-1}(b)\right)$, assume that $\gamma^{u}(t)=\exp (t u)$ and $\gamma^{v}(t)=\exp (t v)$. By Lemma 3.3.2 there exists $\epsilon>0$ such that for every $t \in(-\epsilon, 0)$,

$$
f\left(\gamma^{u}(t)\right)=f\left(\gamma^{v}(t)\right)
$$

Because from this Lemma we have that $f^{-1}(c)=C_{r_{c, b}^{-}}^{-}\left(f^{-1}(b)\right)$, for every $c \in(a, b)$. Now by the definition of the past cylinder, $\gamma^{u}$ and $\gamma^{v}$ are the extensions of the minimizing geodesics joining $f^{-1}(c)$ to $f^{-1}(b)$. So we have that for $t \in\left(-r_{c, b}^{-}, 0\right), f\left(\gamma^{u}(t)\right)=f\left(\gamma^{v}(t)\right)$. Set $g: \mathbb{R} \longrightarrow \mathbb{R}$ with $t \longmapsto f\left(\gamma^{u}(t)\right)-f\left(\gamma^{v}(t)\right)$ and notice that $g$ is analytic. Hence being analytical together with $\left.g\right|_{\left(-r_{a, b}^{-}, 0\right)}=0$ implies that $g \equiv 0$ completing the proof.

Claim 3.3.6. Given $c \in(a, b), C_{r_{c}}^{+}\left(f^{-1}(b)\right) \subset f^{-1}(c)$, where $r_{c}=r_{c, b}^{+}=d_{F}\left(f^{-1}(b), f^{-1}(c)\right)$.

Proof. Consider a unitary speed geodesic $\gamma$ joining $f^{-1}(b)$ to $f^{-1}(c)$ with

$$
L(\gamma)=d_{F}\left(f^{-1}(b), f^{-1}(c)\right)=r_{c} .
$$

Now for every $y \in C_{r_{c}}^{+}\left(f^{-1}(b)\right)$ there exists a geodesic $\gamma_{y}$ such that $\gamma_{y}^{\prime}(0) \in \mathfrak{C}_{\gamma_{y}(0)}^{1}\left(f^{-1}(b)\right)$ and $\gamma_{y}\left(r_{c}\right)=y$. By Statement 3.3.5,

$$
c=f\left(\gamma\left(r_{c}\right)\right)=f\left(\gamma_{y}\left(r_{c}\right)\right)
$$

meaning that $y \in f^{-1}(c)$ and it concludes the proof.

Statement 3.3.7. Given $c \in[a, b]$, if $u \in \mathfrak{C}^{1}\left(f^{-1}(b)\right)$, then $\gamma^{u}(t)=\exp (t u)$, for $t \in\left[0, r_{b, a}^{+}\right]$meets $f^{-1}(c)$ exactly once that happens at time $r_{c}$ where $r_{c}=d_{F}\left(f^{-1}(b), f^{-1}(c)\right)$.

Proof. Define $\quad \mathfrak{R}:\left[c_{0}, c_{1}\right] \longrightarrow \mathbb{R}, a<c_{0}<c_{1}<b$,

$$
c \rightarrow d_{F}\left(f^{-1}(b), f^{-1}(c)\right)=r_{c} \text {. }
$$




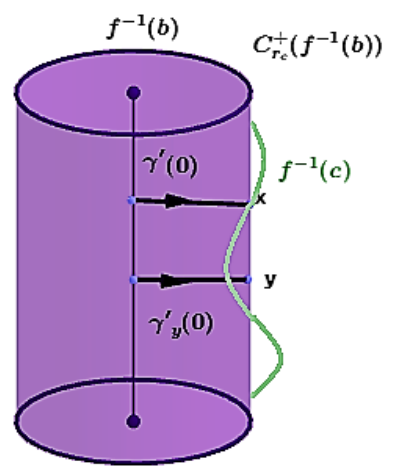

Figure 3.6: Claim 3.3.6

Subclaim 3.3.8. IF $c<\tilde{c}$, then $\mathfrak{R}(c)>\mathfrak{R}(\tilde{c})$.

Proof. For $c$, since $f^{-1}(c)$ and $f^{-1}(b)$ are compact submanifolds, there exists a unitary speed geodesic $\gamma$ joining $f^{-1}(b)$ to $f^{-1}(c)$ with

$$
L\left(\left.\gamma\right|_{\left[0, r_{c}\right]}\right)=d_{F}\left(f^{-1}(b), f^{-1}(c)\right)=r_{c} .
$$

Therefore there exists at least one time $t_{0} \in\left(0, r_{c}\right)$ such that $\gamma\left(t_{0}\right) \in f^{-1}(\tilde{c})$. So

$$
\mathfrak{R}(\tilde{c})=d_{F}\left(f^{-1}(b), f^{-1}(\tilde{c})\right) \leq L\left(\left.\gamma\right|_{\left[0, t_{0}\right]}\right)=t_{0}<\mathfrak{R}(c) .
$$

Consider the geodesic $\gamma$ as it is in Subclaim 3.3.8. According to relation 3.5 and Subclaim 3.3.8 we have the following facts:

$$
d_{F}\left(f^{-1}(b), \gamma\left(r_{\tilde{c}}\right)\right)=r_{\tilde{c}},
$$

for $r_{\tilde{c}} \leq r_{c}$. Because otherwise $d_{F}\left(f^{-1}(b), f^{-1}(c)\right)<r_{c}$. Now EQ. 3.6, Claim 3.3.6, and the definition of future cylinder imply the following equation

$$
\gamma\left(r_{\tilde{c}}\right) \in C_{r_{\tilde{c}}}^{+}\left(f^{-1}(b)\right) \subset f^{-1}(\tilde{c}),
$$

where in the inclusion we used Claim 3.3.6. EQ. 3.7 together with Subclaim 3.3.8 guarantee that $\left.\gamma\right|_{\left[0, r_{c}\right]}$ crosses each leaf $f^{-1}(\tilde{c})$ just once that happens at time $r_{\tilde{c}}$. Observe that as $\left.\gamma\right|_{\left[0, r_{c}\right]}$ minimizes the distance from $f^{-1}(b)$ to $f^{-1}(c)$, by Lemma 1.3.13, we have that $\gamma$ is orthogonal to $f^{-1}(b)$ and $f^{-1}(c)$. As a result from above explanation and Statement 3.3.5 we have that for every $u \in \mathfrak{C}^{1}\left(f^{-1}(b)\right), \gamma^{u}(t)=\exp (t u)$, for $t \in\left[0, r_{c}\right]$, meets each leaf $f^{-1}(\tilde{c})$ exactly once that happens at time $r_{\tilde{c}}$.

Remark 3.3.9. By Statement 3.3.7 for every $u \in \mathfrak{C}^{1}\left(f^{-1}(b)\right)$ the geodesic $\gamma^{u}(t)=\exp (t u)$ meets $f^{-1}(c)$ at time $r_{r}=r_{c, b}^{+}$and so it minimizes the distance between $f^{-1}(b)$ and $f^{-1}(c)$ and consequently by Lemma 1.3 .13 it is orthogonal to $f^{-1}(c)$.

Claim 3.3.10. If $c$ is a regular value then

$$
C_{r_{c}}^{+}\left(f^{-1}(b)\right)=f^{-1}(c),
$$


where $r_{c}=d_{F}\left(f^{-1}(b), f^{-1}(c)\right)$.

Proof. First proof: According to Claim 3.3.6 we have one inclusion. Let us prove the other inclusion. By compactness of $f^{-1}([c, b])$ in $M$, there exists a finite partition of $[c, b]$ by $c=c_{k}<$ $c_{k-1}<\ldots<c_{1}<c_{0}=b$ such that

$$
f^{-1}\left(c_{i+1}\right) \subset T u b_{r_{i}}^{+}\left(f^{-1}\left(c_{i}\right)\right)
$$

for some $r_{i}>0$, where $i=0, \ldots, k-1$. Now for $y \in f^{-1}(c)$, let $\gamma_{k}$ be the unitary speed geodesic such that

$$
L\left(\left.\gamma_{k}\right|_{\left[0, r_{c_{k}}\right]}\right)=d_{F}\left(f^{-1}\left(c_{k-1}\right), y\right)=r_{c_{k}} .
$$

Let $\gamma_{i}$ be the unitary speed geodesic such that

$$
L\left(\left.\gamma_{i}\right|_{\left[0, r_{c_{i}}\right]}\right)=d_{F}\left(f^{-1}\left(c_{i-1}\right), \gamma_{i+1}(0)\right)=r_{c_{i}} .
$$

Observe that by Lemma 1.3.13, $\gamma_{i}^{\prime}(0) \in \mathfrak{C}^{1}\left(f^{-1}\left(c_{i-1}\right)\right)$, for every $i \in(1, k)$. Moreover as $f^{-1}\left(c_{i-1}\right)$ has codimension $1, \gamma_{i}^{\prime}(0)$ is in the opposite side or in the same side of $\nabla f$ with respect to the hyperplane $T_{\gamma_{i}(0)} f^{-1}\left(c_{i-1}\right)$. Since $\left.\gamma_{i}\right|_{\left[0, r_{c_{i}}\right]}$ is minimizing, it must be in the opposite side. In addition, as

$$
f^{-1}\left(c_{i+1}\right) \subset T u b_{r_{i}}^{+}\left(f^{-1}\left(c_{i}\right)\right)
$$

$\left.\gamma_{i}\right|_{\left[0, r_{c_{i}}\right]}$ intersect $f^{-1}\left(c_{i+1}\right)$ just once which happens at time $r_{c_{i}}$. Now consider the concatenation of these geodesic as

$$
\bar{\gamma}=\gamma_{1} * \gamma_{2} * \ldots * \gamma_{k} .
$$

Then $\left.\bar{\gamma}\right|_{[0, r]}$, where $r=\sum_{i=1}^{k} r_{c_{i}}$, is a geodesic that joins $f^{-1}(b)$ to $y=\bar{\gamma}(r)$ and $\bar{\gamma}^{\prime}(0) \in \mathfrak{C}^{1}\left(f^{-1}(b)\right)$. By construction $\left.\bar{\gamma}\right|_{[0, r]}$ crosses each leaf $f^{-1}\left(c_{i}\right)$ just once at time $r_{c_{i}}$. As with respect to Statement 3.3.7, $\left.\bar{\gamma}\right|_{[0, r]}$ meets $f^{-1}(c)$ just once at time $r_{c}$, we must have $r=r_{c}$. Notice that if $\beta$ is another geodesic with

$$
d_{F}\left(f^{-1}(b), y\right)=L\left(\left.\beta\right|_{[0, t]}\right)=t<r_{c},
$$

by Lemma 1.3 .13 we have $\beta^{\prime}(0) \in \mathfrak{C}^{1}\left(f^{-1}(b)\right)$ and then by Claim 3.3.5 we have that $\bar{\gamma}(t) \in f^{-1}\left(c_{k}\right)$ that is a contradiction. Consequently

$$
d_{F}\left(f^{-1}(b), y\right)=L\left(\left.\bar{\gamma}\right|_{\left[0, r_{c}\right]}\right)=r_{c}
$$

that means $y \in C_{r_{c}}^{+}\left(f^{-1}(b)\right)$, providing the other inclusion.

Second proof: Given $c \in(a, b)$, by Claim 3.3.6, $C_{r_{c}}^{+}\left(f^{-1}(b)\right)$ is contained in $f^{-1}(c)$. Also as $c$ is a regular value, $f^{-1}(c)$ is of codimension 1 . So in order to conclude the proof that is enough to show that $C_{r_{c}}^{+}\left(f^{-1}(b)\right)$ is a submanifold of codimension 1 . First choose $c_{1}$ in such a way that

$$
f^{-1}\left(c_{1}\right) \subset T u b_{r_{0}}^{+}\left(f^{-1}(b)\right)
$$

for some $r_{0}>0$. By compactness of $f^{-1}\left(\left[c, c_{1}\right]\right)$ in $M$, there exists a finite partition of $\left[c, c_{1}\right]$ by $c=c_{k}<c_{k-1}<\ldots .<c_{1}<b$ such that

$$
f^{-1}\left(c_{i+1}\right) \subset T u b_{r_{i}}^{+}\left(f^{-1}\left(c_{i}\right)\right),
$$


for some $r_{i}>0$. As $f^{-1}\left(c_{1}\right) \subset T u b_{r_{0}}^{+}\left(f^{-1}(b)\right)$, by Lemma 1.4.23, $C_{r_{c_{1}}}^{+}\left(f^{-1}(b)\right)$ is a submanifold of codimension 1 and so

$$
C_{r_{c_{1}}}^{+}\left(f^{-1}(b)\right)=f^{-1}\left(c_{1}\right) .
$$

Now for $y \in C_{r_{c_{1}}}^{+}\left(f^{-1}(b)\right)$, let $\gamma_{1}$ be the unitary speed geodesic such that

$$
L\left(\left.\gamma_{1}\right|_{\left[0, r_{c_{1}}\right]}\right)=d_{F}\left(f^{-1}(b), y\right)=r_{c_{1}} .
$$

From Statement 3.3 .7 we have $\gamma_{1}^{\prime}\left(r_{c_{1}}\right) \in \mathfrak{C}^{1}\left(f^{-1}\left(c_{1}\right)\right)$. So there exists a unitary vector field $\xi_{1}$ orthogonal to $f^{-1}\left(c_{1}\right)$ with $\xi_{1}(y)=\gamma_{1}^{\prime}\left(r_{c_{1}}\right)$. Define

$$
\begin{aligned}
\eta_{\xi_{1}}^{+}: & f^{-1}\left(c_{1}\right) \longrightarrow f^{-1}\left(c_{2}\right) \\
& x \mapsto \exp \left(r_{c_{2}}-r_{c_{1}}\right) \xi_{1}(x) .
\end{aligned}
$$

First, from statement 3.3.7, this map is well-defined. Moreover, since $f^{-1}\left(c_{2}\right) \subset T u b_{r_{1}}^{+}\left(f^{-1}\left(c_{1}\right)\right)$, this map is a diffeomorphism on its image. Consequently $C_{r_{c_{2}}}^{+}\left(f^{-1}(b)\right)$ is a submanifold of codimension 1 which means

$$
C_{r_{c_{2}}}^{+}\left(f^{-1}(b)\right)=f^{-1}\left(c_{1}\right)
$$

By the same argument, mutatis mutandis, one can define

$$
\begin{aligned}
\eta_{\xi_{i}}^{+}: & f^{-1}\left(c_{i}\right) \longrightarrow f^{-1}\left(c_{i+1}\right) \\
& x \mapsto \exp \left(r_{c_{i+1}}-r_{c_{i}}\right) \xi_{i}(x),
\end{aligned}
$$

where $\xi_{i} \in \mathfrak{C}^{1}\left(f^{-1}\left(c_{i}\right)\right)$, and show that $C_{r_{c_{i+1}}}^{+}\left(f^{-1}(b)\right)$ is a submanifold of codimension 1 providing that

$$
C_{r_{c_{i+1}}}^{+}\left(f^{-1}(b)\right)=f^{-1}\left(c_{i}\right)
$$

Proof of Lemma 3.3.4.

Choose $c$ in such a way that $f^{-1}(c) \subset T u b_{r}^{-}\left(f^{-1}(a)\right)$, for some $r>0$. Firstly, by Claim 3.3.10, $f^{-1}(c)=C_{r_{c}}^{+}\left(f^{-1}(b)\right)$. So for every $x \in f^{-1}(c)$, by Remark 3.3.9

$$
T_{x} f^{-1}(c)=\left\{v \in T_{x} M: g_{\gamma_{x}^{\prime}\left(r_{c}\right)}\left(\gamma_{x}^{\prime}\left(r_{c}\right), v\right)=0\right\},
$$

where $\gamma_{x}^{\prime}(0) \in \mathfrak{C}_{\gamma_{x}(0)}^{1}\left(f^{-1}(b)\right)$ with $\gamma_{x}\left(r_{c}\right)=x$. Observe that we also have that $\gamma_{x}^{\prime}\left(r_{c}\right) \in \mathfrak{C}_{\gamma_{x}\left(r_{c}\right)}^{1}\left(f^{-1}(c)\right)$. Secondly, from $f^{-1}(c) \subset T u b_{r}^{-}\left(f^{-1}(a)\right)$ we have that

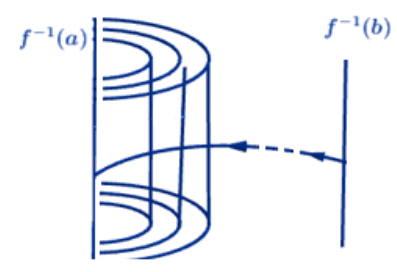

Figure 3.7 


$$
T_{x} C_{\left(r_{a}-r_{c}\right)}^{-}\left(f^{-1}(a)\right)=\left\{v \in T_{x} M: g_{\gamma_{x}^{\prime}\left(r_{c}\right)}\left(\gamma_{x}^{\prime}\left(r_{c}\right), v\right)=0\right\}
$$

Hence $T_{x} C_{\left(r_{a}-r_{c}\right)}^{-}\left(f^{-1}(a)\right)=T_{x} f^{-1}(c)$ and as we have this equality of an arbitrary $x \in f^{-1}(c)$, by unicity of the foliation the leaves coincide.

Now as $f^{-1}(c)$ is parallel to $f^{-1}(d)$ for all values $c, d$ with $c<d$, we get what we desired.

Proving this lemma we accomplished proving the Theorem.

Remark 3.3.11. From Theorem 3.3 .1 one can say that for every point belongs to $f^{-1}(c)$ there exists a minimizing geodesic emanating from that point which is orthogonal to $f^{-1}(c)$ and this geodesic meets each regular leaf twice orthogonally and each singular leaves once orthogonally.

\subsubsection{Example}

Example 3.3.12. Let us recall Example 2.4.6 of Chapter 2. Consider the Euclidean space $\left(\mathbb{R}^{n+1}, h\right)$, in which $h$ is the canonical metric, with the Killing vector field $W$. It is worth mentioning that one can put any other wind $W$ which is projectable. Now consider the function $f: \mathbb{R}^{n+1} \longrightarrow \mathbb{R}$ with $f\left(x_{1}, \ldots, x_{n+1}\right)=x_{n+1}$ and define $\tilde{f}:=\left.f\right|_{S^{n}}$. Observe that $\tilde{f}\left(S^{n}\right)=[-1,1], \tilde{f}$ is analytic and we have a foliation $\mathcal{F}=\left\{f^{-1}(c)\right\}_{c \in[-1,1]} \cap S^{n}$. Let us calculate $F(\nabla \tilde{f})$ in which $\nabla \tilde{f}$ is the gradient of $\tilde{f}$ with respect to $F$. Note that $W$ is tangent to $S^{n}$ and also tangent to the leaves. Because

$$
\operatorname{gradf}=\bar{\nabla} \tilde{f}+\langle\operatorname{gradf}, p\rangle p
$$

in which gradf is the gradient of $f$ with respect to the canonical metric $h, \bar{\nabla} \tilde{f}$ is the gradient of $\tilde{f}$ with respect to $\left.h\right|_{S^{n}}$, and $p \in S^{n}$. Therefore

$$
\bar{\nabla} \tilde{f}=(0, \ldots, 0,1)-\tilde{f} \cdot p
$$

which implies that $\langle\bar{\nabla} \tilde{f}, W\rangle=0$. So one can apply Lemma 2.2.10 which says $F(\nabla \tilde{f})=\|\bar{\nabla} \tilde{f}\|$ and so it is sufficient to calculate $\|\bar{\nabla} \tilde{f}\|$. Since we wrote gradf as the orthogonal decomposition, we have

$$
\|\operatorname{gradf}\|^{2}=\|\bar{\nabla} \tilde{f}\|^{2}+\langle\operatorname{gradf}, p\rangle^{2}\|p\|^{2}
$$

which implies that $\|\bar{\nabla} \tilde{f}\|=\sqrt{1-\tilde{f}^{2}}$ which means for $\mathfrak{b}=1-t^{2}, F^{2}(\nabla \tilde{f})=\mathfrak{b} \circ \tilde{f}$. As a consequence $f$ is $F$-transnormal with $\tilde{f}^{-1}(-1)$ and $\tilde{f}^{-1}(1)$ as the singular leaves of the singular foliation $\mathcal{F}=$ $\left\{f^{-1}(c)\right\}_{c \in[-1,1]} \cap S^{n}$. Here all of the conditions of Theorem 3.3.1 are satisfied and one can apply it here. Moreover note that according to Lemma 2.2.10, in the case of interest, one also can calculated

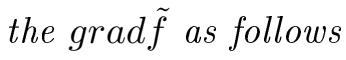

$$
\nabla \tilde{f}=\bar{\nabla} \tilde{f}+\| \bar{\nabla} \tilde{f}|| W=e_{n+1}-\tilde{f} \cdot p+\sqrt{1-\tilde{f}^{2}} W
$$

By Theorem 3.3.1, for $c, d \in[-1,1], f^{-1}(c)$ is equidistant to $f^{-1}(d)$ that is we have $f^{-1}(c) \| f^{-1}(d)$ and $f^{-1}(d) \| f^{-1}(c)$.

Let's see a special case and calculate the distance between two leaves using the orthogonal 
geodesics. Let $n=2$ and

$$
W(p)=p\left(\begin{array}{ccc}
0 & \frac{1}{2} & 0 \\
-\frac{1}{2} & 0 & 0 \\
0 & 0 & 0
\end{array}\right)
$$

Then the flow of $W$ is as

$$
\varphi(t, p)=p\left(\begin{array}{ccc}
\cos \frac{t}{2} & \sin \frac{t}{2} & 0 \\
-\sin \frac{t}{2} & \cos \frac{t}{2} & 0 \\
0 & 0 & 1
\end{array}\right)
$$

By Lemma 2.2.14, given a h-geodesic $\alpha$, the corresponding F-geodesic is as $\gamma(t)=\varphi(t, \alpha(t))$. Now let us be more specific and consider the point $p=(1,0,0)$ and two vectors $v_{1}=(0,0,1)$ and $v_{2}=(0,0,-1)$. Suppose that $\alpha_{1}(t)$ and $\alpha_{2}(t)$ are two geodesics in $S^{2}$ with respect to metric $\left.h\right|_{S^{2}}$ with the initial velocities $v_{1}$ and $v_{2}$ which emanate from $p$. That is $\alpha_{1}(0)=\alpha_{2}(0)=p$ and $\alpha_{1}^{\prime}(0)=v_{1}$ and $\alpha_{2}^{\prime}(0)=v_{2}$. Put $\gamma_{1}(t)=\varphi\left(t, \alpha_{1}(t)\right)$ and $\gamma_{2}(t)=\varphi\left(t, \alpha_{2}(t)\right)$. Observe that

$$
\alpha_{1}(t)=\cos t p+\sin t v_{1}=(\cos t, 0, \sin t), \quad \gamma_{1}(t)=(\cos t, 0, \sin t)\left(\begin{array}{ccc}
\cos \frac{t}{2} & \sin \frac{t}{2} & 0 \\
-\sin \frac{t}{2} & \cos \frac{t}{2} & 0 \\
0 & 0 & 1
\end{array}\right)
$$

and

$$
\alpha_{2}(t)=\cos t p+\sin t v_{2}=(\cos t, 0,-\sin t), \quad \gamma_{2}(t)=(\cos t, 0,-\sin t)\left(\begin{array}{ccc}
\cos \frac{t}{2} & \sin \frac{t}{2} & 0 \\
-\sin \frac{t}{2} & \cos \frac{t}{2} & 0 \\
0 & 0 & 1
\end{array}\right)
$$

At point $p$ we have

$$
W=\left(0, \frac{1}{2}, 0\right), \gamma_{1}^{\prime}(0)=\left(0, \frac{1}{2}, 1\right), \gamma_{2}^{\prime}(0)=\left(0, \frac{1}{2},-1\right) \text { and } F\left(\gamma_{1}^{\prime}(0)\right)=1=F\left(\gamma_{2}^{\prime}(0)\right) .
$$

As for $L_{p}=\tilde{f}^{-1}(0)$ we have $T_{p}\left(L_{p}\right)=\mathbb{R} . u$, where $u=(0,1,0)$, then

$$
h\left(u, \gamma_{1}^{\prime}(0)-W\right)=0=h\left(u, \gamma_{2}^{\prime}(0)-W\right)
$$

Comparing two last equalities with Corollary 2.2.7 we have that $\gamma_{1}^{\prime}(0)$ and $\gamma_{2}^{\prime}(0) \in \mathfrak{C}_{p}\left(L_{p}\right)$ and so according to Theorem 3.3.1 they remain orthogonal to all the leaves. Note that $\gamma_{1}(t)$ coincides with the integral curve of $\nabla \tilde{f}$ as it is expected. According to above explanation, $\gamma_{1}(t)$ and $\gamma_{2}(t)$ are orthogonal to all the leaves and so they are minimizing geodesics. In particular $\gamma_{1}(t)$ minimizes the distance from $L_{p}=\tilde{f}^{-1}(0)$ to $L_{q_{1}}=\tilde{f}^{-1}\left(\frac{\sqrt{2}}{2}\right)$ in which $q_{1}=\gamma_{1}\left(\frac{\pi}{4}\right)$ and $\gamma_{2}(t)$ minimizes the distance from $L_{q_{2}}=\tilde{f}^{-1}\left(\frac{\sqrt{2}}{2}\right)$ to $L_{p}=\tilde{f}^{-1}(0)$ in which $q=\gamma_{2}\left(-\frac{\pi}{4}\right)$. Now according to Theorem 3.3.1, $L_{p}$ and $L_{q_{1}}=L_{q_{2}}$ are equidistant and we have

$$
d_{F}\left(L_{p}, L_{q_{1}}\right)=d_{F}\left(p, q_{1}\right)=\int_{0}^{\frac{\pi}{4}} F\left(\gamma_{1}^{\prime}(t)\right) d t=\frac{\pi}{4}
$$

and

$$
d_{F}\left(L_{q_{2}}, L_{p}\right)=d_{F}\left(q_{1}, p\right)=\int_{-\frac{\pi}{4}}^{0} F\left(\gamma_{2}^{\prime}(t)\right) d t=\frac{\pi}{4} .
$$


Note that as $\gamma_{i}$ is a geodesic, it has constant velocity and for every $t, F\left(\gamma_{i}^{\prime}(t)\right)=F\left(\gamma_{i}^{\prime}(0)\right)=1$. As in this example $S^{2}$ is homogeneous and the vector field of the wind in tangent to the leaves we have that $d_{F}\left(L_{p}, L_{q}\right)=d_{F}\left(L_{q}, L_{p}\right)$. Observe that calculating the distance between two leaves demands

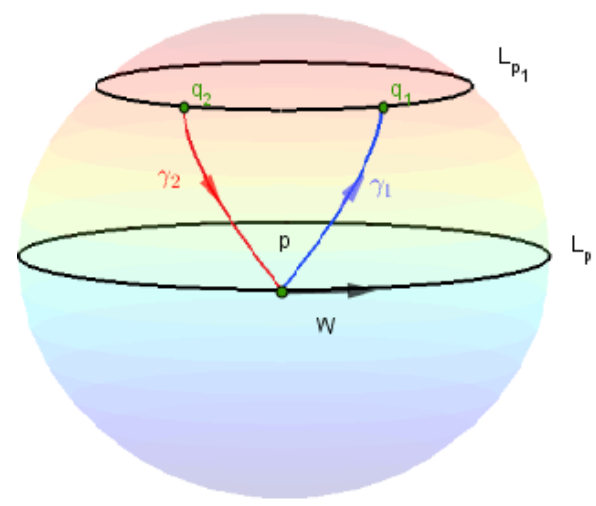

Figure 3.8: The equidistant leaves

some work and sometimes is quite tedious. Because of this this is more reasonable to use the other possible ways. One of them is the way provided by Proposition 3.2.1. That is for the case that $c<d$,

$$
d_{F}\left(\tilde{f}^{-1}(c), q\right)=d_{F}\left(\tilde{f}^{-1}(c), \tilde{f}^{-1}(d)\right)=\int_{c}^{d} \frac{d s}{\sqrt{\mathfrak{b}(s)}} .
$$

Using this formula one has

$$
d_{F}\left(L_{p}, L_{q_{1}}\right)=d_{F}\left(\tilde{f}^{-1}(0), \tilde{f}^{-1}\left(\frac{\sqrt{2}}{2}\right)\right)=\int_{0}^{\frac{\sqrt{2}}{2}} \frac{d s}{\sqrt{1-s^{2}}}=\frac{\pi}{4},
$$

which is the same answer that we already calculated using the minimizing geodesic. To calculate $d_{F}\left(L_{q_{1}}, L_{p}\right)$ there is a formula that will be given in Proposition 3.5.10.

\subsection{Answering Question 3.1.3}

\subsubsection{Proposition and proof}

In this section we answer Question 3.1.3 positively. In this case a proposition is stated that gives the necessary conditions with which a foliation can be Riemannian. Two different proofs are provided for this proposition. The first proof is based on some properties of Hessian. In the second proof we proof the proposition based on another result from [AAJ17]. In both the proofs we use Theorem 1.3.38 from [QT15].

Proposition 3.4.1. Let $(M, F)$ be a compact Finsler manifold and $f: M \longrightarrow[a, b]$ be a Ftransnormal function with $F^{2}(\nabla f)=\mathfrak{b}(f)$, where $\mathfrak{b}$ is a $C^{1}$ function on $[a, b]$, such that

(a) $\mathcal{F}=\left\{f^{-1}(c)\right\}_{c \in[a, b]}$ is a singular foliation,

(b) $a$ and $b$ are the only singular values at $[a, b]$,

(c) $\mathfrak{b}^{\prime}(a) \neq 0 \neq \mathfrak{b}^{\prime}(b)$, 
(d) and each of $f^{-1}(a)$ and $f^{-1}(b)$ has codimension greater than one.

Then there exists a Riemannian metric on $M$ such that $\mathcal{F}=\left\{f^{-1}(c)\right\}_{c \in[a, b]}$ is a SRF.

Remark 3.4.2. Observe that in the hypothesis of this theorem, compactness and items $(b)$ and $(d)$ are the necessary conditions for Theorem 1.3 .38 and we do not use them directly during the proof.

Proof. Given $q \in f^{-1}(a)$ let $S_{q}$ be the slice at $q$ with respect to some Riemannian metric $h$ on $M$. That is

$$
S_{q}=\exp _{q}^{\perp}\left(T_{q}^{\perp} f^{-1}(a)\right)
$$

where $\exp _{q}$ is the exponential map at $q$ with respect to $h$ and $\exp _{q}^{\perp}:=\left.\exp _{q}\right|_{T_{q}^{\perp} f^{-1}(a)}$. Let $\gamma$ be a unit speed geodesic which is orthogonal to $f^{-1}(a)$, that is $\gamma^{\prime}(0) \in \mathfrak{C}^{1}\left(f^{-1}(a)\right)$. Then according to Proposition 3.2.1, $\gamma$ is an extension of the integral curve of the gradient of $f, \nabla f$, up to a reparametrization. Indeed for $t>0, \gamma$ can be written as the curve $t \rightarrow \gamma\left(\frac{t}{F(\nabla f)}\right)$ and so

$$
\gamma^{\prime}=\frac{\nabla f(\gamma)}{F(\nabla f)}
$$

Consider the decomposition

$$
T_{q} M=T_{q} f^{-1}(a) \bigoplus T_{q}^{\perp} f^{-1}(a)
$$

at $q$ given by the Riemannian metric $h$. Then $\gamma^{\prime}(0)=\gamma_{s}^{\prime}(0)+\gamma_{t}^{\prime}(0)$, where $\gamma_{t}^{\prime}(0) \in T_{q} f^{-1}(a)$ and $\gamma_{s}^{\prime}(0) \in T_{q}^{\perp} f^{-1}(a)$. As Hessian is a bilinear and symmetric operator and also using item $(a)$ of Lemma 1.3.44 one has

$$
\text { Hess } \begin{aligned}
f_{q}\left(\gamma^{\prime}(0), \gamma^{\prime}(0)\right) & =\operatorname{Hess} f_{q}\left(\gamma_{s}^{\prime}(0), \gamma_{s}^{\prime}(0)\right)+2 \operatorname{Hess} f_{q}\left(\gamma_{s}^{\prime}(0), \gamma_{t}^{\prime}(0)\right)+\operatorname{Hess} f_{q}\left(\gamma_{t}^{\prime}(0), \gamma_{t}^{\prime}(0)\right) \\
& =\operatorname{Hess} f_{q}\left(\gamma_{s}^{\prime}(0), \gamma_{s}^{\prime}(0)\right) \\
& =\operatorname{Hess}^{\perp} f_{q}\left(\gamma_{s}^{\prime}(0), \gamma_{s}^{\prime}(0)\right),
\end{aligned}
$$

where Hess ${ }^{\perp} f_{q}=\left.\operatorname{Hess} f_{q}\right|_{T_{q}^{\perp} f^{-1}(a)}$ (see EQ. 1.12). Moreover by EQ. 3.10 and Lemma 1.3.41, given $c_{0} \in(a, b)$, for every $t \in\left(0, d_{F}\left(f^{-1}(a), f^{-1}\left(c_{0}\right)\right)\right)$, we have

$$
\operatorname{Hess} f\left(\gamma^{\prime}(t), \gamma^{\prime}(t)\right)=\frac{\operatorname{Hess} f(\nabla f(\gamma), \nabla f(\gamma))}{F^{2}(\nabla f)}=\frac{1}{2} \mathfrak{b}^{\prime}(f(\gamma))
$$

Now by continuity, from EQs. 3.12 and 3.11, we have

$$
\begin{aligned}
\operatorname{Hess}^{\perp} f_{q}\left(\gamma_{s}^{\prime}(0), \gamma_{s}^{\prime}(0)\right) & =\operatorname{Hess} f_{q}\left(\gamma^{\prime}(0), \gamma^{\prime}(0)\right)=\lim _{t \rightarrow 0} \operatorname{Hess} f\left(\gamma^{\prime}(t), \gamma^{\prime}(t)\right)=\lim _{t \rightarrow 0} \frac{1}{2} \mathfrak{b}^{\prime}(f(\gamma)) \\
& =\frac{1}{2} \mathfrak{b}^{\prime}(a) .
\end{aligned}
$$

Applying the hypothesis, from EQ. 3.13 we have

$$
\operatorname{Hess}^{\perp} f_{q}\left(\gamma_{s}^{\prime}(0), \gamma_{s}^{\prime}(0)\right) \neq 0
$$

which means that the normal Hessian is non-degenerate at $q$.

Now given $q \in f^{-1}(b)$, let $\tilde{S}_{q}$ be the associated slice. Let $\gamma$ be a unit speed geodesic which is toward $f^{-1}(b)$ and meets it orthogonally at some time $r$. Then $\gamma$ is an extension of the integral 
curve of the gradient of $f$, up to a reparametrization. Then again by the same argument as above, Mutatis Mutandis, one has

$$
\operatorname{Hess}^{\perp} f_{q}\left(\gamma^{\prime}(r), \gamma^{\prime}(r)\right)=\frac{1}{2} \mathfrak{b}^{\prime}(b) \neq 0
$$

which means that the normal Hessian is non-degenerate at $q$.

With respect to EQs. 3.14 and 3.15, at each critical point $q$, the normal Hessian $\operatorname{Hess}^{\perp} f_{q}$ is non-degenerate and so by Definition 1.3.37, $f$ is a Bott-Morse function. As a result, with respect to Theorem 1.3.38, there exists a Riemannian metric on $M$ so that $f$ is transnormal. Now one can apply Wang's Theorem which says that $\mathcal{F}=\left\{f^{-1}(c)\right\}_{c \in[a, b]}$ is a singular Riemannian foliation.

Before proceeding with the second proof, we shall state a proposition based on the proof will be given.

Proposition 3.4.3 (Slice Theorem). [AAJ17] Let $\mathcal{F}=\{L\}$ be a SFF on a Finsler manifold $(M, F)$. Given $q \in M$, there exists a slice $S_{q}$ transversal to $L_{q}$ (i.e. $T_{q} M=T_{q} S_{q} \oplus T_{q} L_{q}$ ) and a metric $F_{s}$ on $S_{q}$ with the following properties:

(a) the leaves of the slice foliation $\mathcal{F}_{q}=\mathcal{F} \cap S_{q}$ endow $\left(S_{q}, F_{s}\right)$ of a structure of singular Finsler foliation;

(b) in a neighborhood of $q, \mathcal{F}$ and $\mathcal{F}_{q}$ have the same transnormal geometry. In other words, the distance between two leaves of $\mathcal{F}_{q}$ (with respect to $F_{s}$ ) is the same (with respect to $F$ ) as the distance between the two plaques of $\mathcal{F}$ containing such leaves;

(c) $\mathcal{F}_{q}$ is a SFF of $\left(S_{q}, F_{s}\right)$.

Proposition 3.4.4. Let $(M, F)$ be a compact Finsler manifold and $f: M \longrightarrow[a, b]$ be a Ftransnormal function with $F^{2}(\nabla f)=\mathfrak{b}(f)$, where $\mathfrak{b}$ is a $C^{1}$ function on $[a, b]$, such that

(a) $\mathcal{F}=\left\{f^{-1}(c)\right\}_{c \in[a, b]}$ is a $S F F$,

(b) $a$ and $b$ are the only singular values at $[a, b]$,

(c) $\mathfrak{b}^{\prime}(a) \neq 0 \neq \mathfrak{b}^{\prime}(b)$,

(d) and each of $f^{-1}(a)$ and $f^{-1}(b)$ has codimension greater than one.

Then there exists a Riemannian metric on $M$ such that $\mathcal{F}=\left\{f^{-1}(c)\right\}_{c \in[a, b]}$ is a SRF.

Proof. Given $q \in f^{-1}(a)$ let $S_{q}$ be the slice at $q$ with respect to some Riemannian metric $h$ on $M$. That is

$$
S_{q}=\exp _{q}^{\perp}\left(T_{q}^{\perp} f^{-1}(a)\right)
$$

where $\exp _{q}$ is the exponential map at $q$ with respect to $h$ and $\exp _{q}^{\perp}:=\left.\exp _{q}\right|_{T_{q}^{\perp} f^{-1}(a)}$. Note that as $f^{-1}(a)$ has codimension greater than $1, S_{q}$ is a submanifold of dimension greater than 1 . Indeed $\operatorname{dim} S_{q}=\operatorname{codim} f^{-1}(a)$ and $S_{q} \cap f^{-1}(a)=q$. Let $\left.f\right|_{S_{q}}=f_{s}$ and assume that $f_{s}\left(S_{q}\right)=\left[a, c_{0}\right]$, for some regular value $c_{0}$. With respect to Proposition 3.4.3, there exists a Finsler metric $F_{s}$ on $S_{q}$ for which

$$
\mathcal{F}_{s}:=\left\{f_{s}^{-1}(c)\right\}_{c \in\left[a, c_{0}\right]}=\left\{f^{-1}(c) \cap S_{q}\right\}_{c \in\left[a, c_{0}\right]}
$$


is a singular Finsler foliation with respect to $F_{s}$ and

$$
d_{F_{s}}\left(f_{s}^{-1}(c), f_{s}^{-1}(d)\right)=d_{F}\left(f^{-1}(c), f^{-1}(d)\right),
$$

for every $c, d \in\left[a, c_{0}\right]$. Observe that $f_{s}^{-1}(a)=q$ is a singular submanifold (a submanifold of dimension zero) of $S_{q}$ and in particular from EQ. 3.16 we have that each $f_{s}^{-1}(c)$ is in fact a sphere in $S_{q}$ centered at $q$ and of some positive radius.

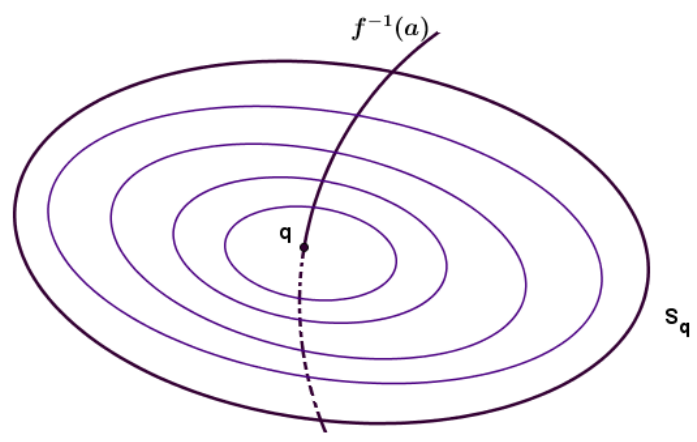

Figure 3.9

Claim 3.4.5. $f_{s}$ is a $F_{s}$-transnormal function on the regular part.

Proof. Given $c, d \in\left(a, c_{0}\right]$ and $x_{i} \in f_{s}^{-1}(c)$, let $\gamma_{x_{i}}$ be the unit speed geodesic for which

$$
d_{F_{s}}\left(x_{i}, f_{s}^{-1}(d)\right)=L\left(\gamma_{x_{i}}\right) .
$$

Then by Lemma 1.3.13 $\gamma_{x_{i}}^{\prime} \in \mathfrak{C}^{1}\left(f_{s}^{-1}(d)\right)$. Since $\mathcal{F}_{s}$ is a singular Finsler foliation, $\gamma_{x_{i}}^{\prime}$ is orthogonal to each leaf it meets. First, as each regular leaf has codimension greater than 1 and, by Comment 3.2.3, $\nabla^{s} f_{s}$ is toward $f_{s}^{-1}\left(c_{0}\right)$ then $\gamma_{x_{i}}$ is a reparametrization of the integral curve of $f_{s}$. That is one has $t \mapsto \gamma_{x_{i}}\left(\frac{\nabla^{s} f_{s}}{F_{s}\left(\nabla^{s} f_{s}\right)}\right)$, where $\nabla^{s} f_{s}$ is the gradient of $f_{s}$ with respect to $F_{s}$. Moreover as the leaves are spheres centered at $q$, then each $\gamma_{x_{i}}$ coincides with some part of the radial geodesic of $S_{q}$ emanating from $q$ and so for every $i, j$ we have

$$
f_{s}\left(\gamma_{x_{i}}(t)\right)=f_{s}\left(\gamma_{x_{j}}(t)\right)
$$

for every $t \in\left(0, d_{F_{s}}\left(f_{s}^{-1}(c), f^{-1}(d)_{s}\right)\right)$. Then

$$
d f_{x_{i}}\left(\frac{\nabla^{s} f_{s}}{F_{s}\left(\nabla^{s} f_{s}\right)}\right)=d f_{x_{j}}\left(\frac{\nabla^{s} f_{s}}{F_{s}\left(\nabla^{s} f_{s}\right)}\right)
$$

which by Definition 1.4.2 means that $\nabla^{s} f_{s}$ is a projectable vector field. Particularly,

$$
F_{s}^{2}\left(\nabla^{s} f_{s}\right)=g_{\nabla^{s} f_{s}}\left(\nabla^{s} f_{s}, \nabla^{s} f_{s}\right)=d f_{x_{i}}\left(\nabla^{s} f_{s}\right)=\mathfrak{b}_{s}\left(f_{s}\right)
$$

where $\mathfrak{b}_{s}:\left(a, c_{0}\right] \longrightarrow \mathbb{R}$ is a smooth function. As a result $f_{s}$ is a $F_{s}$-transnormal function in the regular part.

Claim 3.4.6. $f_{s}$ is $F_{s}$-transnormal on $S_{q}$ and $F_{s}^{2}\left(\nabla^{s} f_{s}\right)=\mathfrak{b}\left(f_{s}\right)$. 


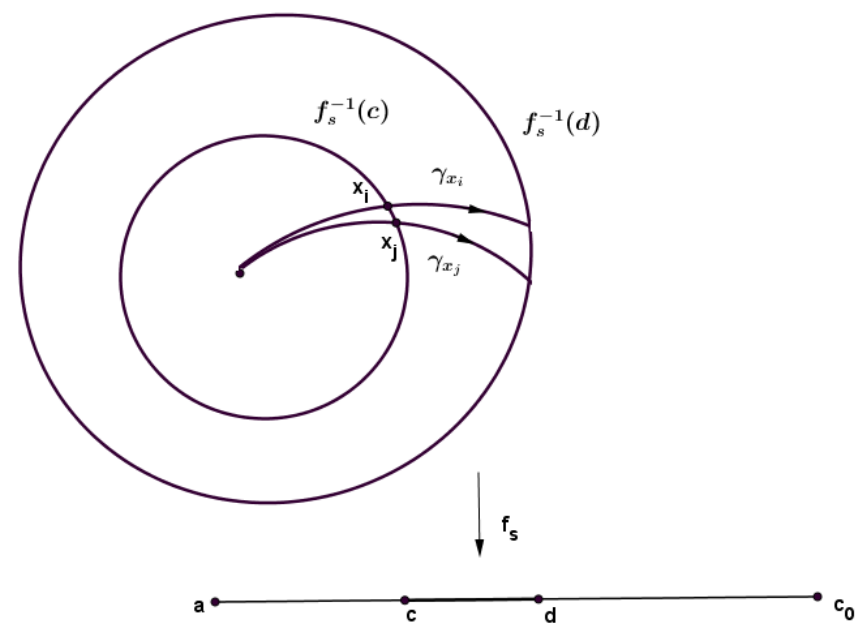

Figure 3.10

Proof. With respect to Claim 3.4.5, $f_{s}$ is $F_{s}$-transnormal on the regular part and $F_{s}^{2}\left(\nabla^{s} f_{s}\right)=\mathfrak{b}_{s}\left(f_{s}\right)$. Here $\mathfrak{b}_{s}$ is continuous on $\left(a, c_{0}\right]$ and smooth on $\left(a, c_{0}\right]$. According to Proposition 3.2.1 and EQ. 3.16, for every $c<d \in\left[a, c_{0}\right]$ we have that

$$
\int_{c}^{d} \frac{d t}{\sqrt{\mathfrak{b}_{s}(t)}}=d_{F_{s}}\left(f_{s}^{-1}(c), f_{s}^{-1}(d)\right)=d_{F}\left(f^{-1}(c), f^{-1}(d)\right)=\int_{c}^{d} \frac{d t}{\sqrt{\mathfrak{b}(t)}}
$$

As a direct consequence of above equality we have that $\mathfrak{b}_{s}(t)=\mathfrak{b}(t)$, for every $t \in\left(0, d_{F_{s}}\left(f^{-1}(a), f^{-1}\left(c_{0}\right)\right)\right]$. Besides this, as $\mathfrak{b}_{s}$ is a continuous function, we also have that $\mathfrak{b}_{s}(0)=\mathfrak{b}(0)$ terminating the proof.

Given a unit vector $v \in T_{q} S_{q} \cap S(1)$, where $S(1)$ is the unit sphere with respect to $F_{s}$ centered at $q$, let $\gamma$ be the geodesic in $S_{q}$ with $\gamma^{\prime}(0)=v$. Then according to Proposition 3.2.1, $\gamma$ is an extension of the integral curve of the gradient of $f_{s}, \nabla^{s} f_{s}$, up to a reparametrization. Indeed for $t>0, \gamma$ can be written as the curve $t \rightarrow \gamma\left(\frac{t}{F_{s}\left(\nabla^{s} f_{s}\right)}\right)$ and so

$$
\gamma^{\prime}=\frac{\nabla^{s} f_{s}(\gamma)}{F_{s}\left(\nabla^{s} f_{s}\right)}
$$

Let Hess ${ }^{\perp} f=$ Hess $\left.f\right|_{T_{q}^{\perp} f^{-1}(a)}$ where we are considering the decomposition $T_{q} M=T_{q} f^{-1}(a) \bigoplus T_{q}^{\perp} f^{-1}(a)$ at $q$ given by the Riemannian metric $h$. By EQ. 3.17 and Lemma 1.3 .41 , for every $t \in\left(0, d_{F_{s}}\left(f^{-1}(a), f^{-1}\left(c_{0}\right)\right)\right)$, we have

$$
\operatorname{Hess}^{\perp} f\left(\gamma^{\prime}, \gamma^{\prime}\right)=\frac{\operatorname{Hess}^{\perp} f_{s}\left(\nabla^{s} f_{s}(\gamma), \nabla^{s} f_{s}(\gamma)\right)}{F_{s}^{2}\left(\nabla^{s} f_{s}\right)}=\frac{1}{2} \mathfrak{b}^{\prime}\left(f_{s}(\gamma)\right) .
$$

Now by continuity, from EQ. 3.18, we have

$$
\begin{aligned}
\operatorname{Hess}^{\perp} f_{s}(v, v) & =\operatorname{Hess}^{\perp} f_{s}\left(\gamma^{\prime}(0), \gamma^{\prime}(0)\right)=\lim _{t \rightarrow 0} \operatorname{Hess}^{\perp} f_{s}\left(\gamma^{\prime}, \gamma^{\prime}\right)=\lim _{t \rightarrow 0} \frac{1}{2} \mathfrak{b}^{\prime}\left(f_{s}(\gamma)\right) \\
& =\frac{1}{2} \mathfrak{b}^{\prime}(a) .
\end{aligned}
$$

Applying the hypothesis, from EQ. 3.19 we have

$$
\operatorname{Hess}^{\perp} f_{s}(v, v) \neq 0
$$


which means that the normal Hessian is non-degenerate at $q$.

Now given $q \in f^{-1}(b)$, let $\tilde{S}_{q}$ be the associated slice. By the same argument as above, Mutatis Mutandis, $f_{\tilde{s}}$ is $F_{\tilde{s}}$-transnormal with $F_{\tilde{s}}^{2}\left(\tilde{\nabla} f_{\tilde{s}}\right)=\mathfrak{b}\left(f_{\tilde{s}}\right)$. Given a unit vector $v \in T_{q} \tilde{S}_{q} \cap \tilde{S}(1)$, where $\tilde{S}(1)$ is the unit sphere with respect to $F_{\tilde{s}}$ in $\tilde{S}_{q}$ centered at $q$, consider $-v$ and let $\gamma$ be the geodesic with $\gamma^{\prime}(0)=-v$. Then $\gamma$ is an extension of the integral curve of the gradient of $f_{\tilde{s}}$, up to a reparametrization. Then again by the same argument as above, Mutatis Mutandis, one has

$$
\operatorname{Hess}^{\perp} f_{\tilde{s}}(v, v)=\operatorname{Hess}^{\perp} f_{\tilde{s}}(-v,-v)=\frac{1}{2} \mathfrak{b}^{\prime}(b)
$$

which means that the normal Hessian is non-degenerate at $q$.

With respect to EQs. 3.20 and 3.21, at each critical point $q$ the normal Hessian $\operatorname{Hess}^{\perp} f_{q}$ is non-degenerate and so by Definition 1.3.37, $f$ is a Bott-Morse function. As a result with respect to Theorem 1.3.38, there exists a Riemannian metric on $M$ so that $f$ is transnormal. Now one can apply Wang's Theorem which says that $\mathcal{F}=\left\{f^{-1}(c)\right\}_{c \in[a, b]}$ is a singular Riemannian foliation.

Remark 3.4.7. The Riemannian metric for which $\mathcal{F}$ is a SRF is the one which is constructed in the proof of Theorem 2.2 in [QT15].

Observe that in the definition of a Finsler transnormal function, the function $\mathfrak{b}$ in

$$
F^{2}(\nabla f)=\mathfrak{b}(f)
$$

is continuous while in the definition of a Riemannian transnormal function, the function $\mathfrak{b}$ in

$$
\|\nabla f\|^{2}=\mathfrak{b}(f)
$$

must be smooth. It means that in previous Theorem $f$ is Riemannian transnormal however the function $\mathfrak{b}$ may not be the same as that one associated to the Finsler metric $F$. Indeed according to the proof of Theorem 2.2 in [QT15], one has $f$ is $h$-transnormal with $\|\bar{\nabla} f\|^{2}=\overline{\mathfrak{b}}(f)$ where

$$
\overline{\mathfrak{b}}(t)= \begin{cases}\frac{4(t-a)}{G^{2}(\sqrt{t-a})} & a \leq t \leq a+\epsilon^{2} \\ 1 & a+\epsilon^{2} \leq t \leq b-\epsilon^{2} \\ \frac{4(b-t)}{G^{2}(\sqrt{b-t})} & b-\epsilon^{2} \leq t \leq b,\end{cases}
$$

for some small $\epsilon>0$ in which

$$
G(r)= \begin{cases}1 & r \leq \frac{\epsilon}{2} \\ 2 r & r \geq \frac{3 \epsilon}{4}\end{cases}
$$

As a consequence we have that for every $c, d \in[a, b]$,

$$
d\left(f^{-1}(c), f^{-1}(d)\right)=d\left(f^{-1}(d), f^{-1}(c)\right)=\int_{c}^{d} \frac{d t}{\sqrt{\overline{\mathfrak{b}}(t)}} .
$$

In particular the Riemannian and Finslerian distances from a leaf $f^{-1}(c)$ to another leaf $f^{-1}(d)$ might be different. Observe that it may happen that for every $c, d \in[a, b]$ the Finslerian distance from $f^{-1}(c)$ to $f^{-1}(d)$ equals the Finslerian distance from $f^{-1}(d)$ to $f^{-1}(c)$, although it does not imply that $F$ is a Riemannian metric. For example see Example 3.3.12. 


\subsubsection{Examples}

Example 3.4.8. Let us recall Example 3.3.12. As it was shown in this example we have a SFF. Since $\mathfrak{b}=1-t^{2}$ is $C^{1}$ and also all of the other hypothesis of Proposition 3.4 .1 are satisfied, there is a Riemannian metric on $S^{n}$ for which $\mathcal{F}=\left\{f^{-1}(c)\right\}_{c \in[-1,1]} \cap S^{n}$ is a singular Riemannian foliation and in particular, by EQs. 3.22 and 3.23, we have

$$
d\left(\tilde{f}^{-1}(0), \tilde{f}^{-1}\left(\frac{\sqrt{2}}{2}\right)\right)=d\left(\tilde{f}^{-1}\left(\frac{\sqrt{2}}{2}\right), \tilde{f}^{-1}(0)\right)=\int_{0}^{\frac{\sqrt{2}}{2}} d s=\frac{\sqrt{2}}{2} .
$$

\subsection{Improvements of some results}

In this section we provide some improvements in Theorem 3.3.1. Observe that in the aforementioned theorem, in hypothesis it was supposed that we are given a singular foliation. In Theorem 3.5.1 below it will be showed that one can even dismiss this assumption. Moreover in Proposition 3.5.10, a formula will be given which together with the formula given in Theorem 3.2.1 one is able to calculate the distances between each two leaves in a SFF $\mathcal{F}=\left\{f^{-1}(c)\right\}_{c \in[a, b]}$.

\subsubsection{Existence of the singular foliation}

Theorem 3.5.1. Let $f: M \longrightarrow \mathbb{R}$ be a F-transnormal and analytic function on a compact Finsler manifold $M$ with $f(M)=[a, b]$ such that the restriction of $f$ on the regular part is a Finsler submersion. Suppose that $a$ and $b$ are the only critical values. Then

(a) each regular level set $f^{-1}(c)$ is equifocal,

(b) the critical level sets $f^{-1}(a)$ and $f^{-1}(b)$ are embedded submanifolds.

Proof.

Proof of item (a). Let us first define the endpoint map and then accomplish the proof through some lemmas. Let $\xi=\frac{\nabla f}{F(\nabla f)}$ be along regular leaves $f^{-1}(c)$. We recall Proposition 3.2.1 and define the endpoint map as $\eta_{r \xi}: f^{-1}(c) \longrightarrow M$ with $x \mapsto \exp _{x}(r \xi)$ where $r \in\left[0, r_{c, b}^{-}\right]$for

$$
r_{c, b}^{-}=d_{F}\left(f^{-1}(c), f^{-1}(b)\right)
$$

Lemma 3.5.2. The map $\eta_{r \xi}: f^{-1}(c) \longrightarrow f^{-1}(d)$ in which $a<c<d<b$ and $r=r_{c, b}^{-}-r_{d, b}^{-}$is $a$ diffeomorphism.

Proof. Firstly, with respect to Proposition 3.2.5, for $r=r_{c, b}^{-}-r_{d, b}^{-}$we have

$$
\eta_{r \xi}\left(f^{-1}(c)\right)=f^{-1}(d)
$$

Next consider $\eta_{-r \xi}^{-}: f^{-1}(d) \longrightarrow M$ with $x \mapsto \gamma(-r)$, where $\gamma(t)=\exp _{x}(t \xi)$. By Proposition 3.2.1, $\eta_{-r \xi}^{-}\left(f^{-1}(d)\right) \subset f^{-1}(c)$. It is straightforwardly checked that

$$
\eta_{-r \xi}^{-} \circ \eta_{r \xi}=\eta_{r \xi} \circ \eta_{-r \xi}^{-}=i d
$$

It deduces that $\eta_{r \xi}$ is a diffeomorphism and $\left(\eta_{-r \xi}^{-}\right)^{-1}=\eta_{r \xi}$. 
Claim 3.5.3. For every $r>r_{c, b}^{-}$, there exists $d \in[a, b]$ such that $\eta_{r \xi}\left(f^{-1}(c)\right) \subset f^{-1}(d)$.

Proof. Observe that given $x \in f^{-1}(c), \eta_{r \xi}(x)=\exp _{x}(r \xi) \in f^{-1}(d)$, for some $d \in[a, b]$. Now set $g: \mathbb{R} \longrightarrow \mathbb{R}$ with $t \mapsto f\left(\eta_{t \xi}(x)\right)-f\left(\eta_{t \xi}(y)\right)$ where $x, y \in f^{-1}(c)$ and $t \in[0, r]$. Note that $g$ is analytic and also by Proposition 3.2.1,

$$
f\left(\eta_{t \xi}(x)\right)=f\left(\eta_{t \xi}(y)\right)
$$

for every $t \in\left[0, r_{c, b}^{-}\right]$. As a result $f\left(\eta_{t \xi}(x)\right)=f\left(\eta_{t \xi}(y)\right)$, for every $t \in\left[r_{c, b}^{-}, r\right]$.

Let $\exp ^{\perp}$ denote the restriction of $\exp$ on $\mathfrak{C}\left(f^{-1}(c)\right)$. We recall that, by Lemma 1.3.25, $z=$ $\exp ^{\perp}\left(r_{0} \xi\right)$ is a $f^{-1}(c)$-focal point with the multiplicity $k$, say $m(z)=k$, if and only if

$$
\operatorname{dim} \operatorname{ker} d\left(\exp ^{\perp}\right)_{r_{0} \xi}=k
$$

One has the following lemma about the $f^{-1}(c)$-focal points.

Lemma 3.5.4. The derivative of map $\eta_{r_{c, b}^{-} \xi}: f^{-1}(c) \longrightarrow f^{-1}(b)$ has constant rank.

Proof. First notice that by Proposition 3.2.1 this map is well-defined and in particular surjective. Now given $p \in f^{-1}(c)$, assume that $\gamma_{p}(t)=\exp _{p}(t \xi)$. Let us first state and prove a claim.

Claim 3.5.5. $\gamma_{p}\left(r_{c, b}^{-}\right)$is a $f^{-1}(c)$-focal point of multiplicity $k$ if and only if $p$ is a critical point of $\eta_{r_{c, b}^{-} \xi}$ and $\operatorname{dim} \operatorname{ker} d\left(\eta_{r_{c, b}^{-}}\right)_{p}=k$.

Proof. Suppose that $w \in \operatorname{ker} d\left(\exp ^{\perp}\right)_{r_{c, b}^{-} \xi}$. So

$$
J\left(r_{c, b}^{-}\right)=d\left(\exp ^{\perp}\right)_{r_{c, b}^{-} \xi}\left(r_{c, b}^{-} w\right)=0 .
$$

Notice that $w \perp \xi=\gamma_{p}^{\prime}(0)$ which means

$$
\operatorname{ker} d\left(\exp ^{\perp}\right)_{r_{c, b}^{-} \xi} \subset \operatorname{ker} d\left(\eta_{r_{c, b}^{-} \xi}\right)_{p}
$$

In other words, $\gamma_{p}\left(r_{c, b}^{-}\right)$is a $f^{-1}(c)$-focal point of multiplicity $k$ if and only if $p$ is a critical point of $\eta_{r_{c, b}^{-} \xi}$ and

$$
\operatorname{dim} \operatorname{ker} d\left(\eta_{r_{c, b}^{-} \xi}\right)_{p}=k
$$

To prove the Lemma, because of the connectedness of the level sets, that is enough to show that for fixed $t$ the rank of $d\left(\eta_{r_{c, b}^{-} \xi}\right)$ is locally constant.

Observe that because $f$ is a Finsler submersion on the regular part, there exists $\epsilon_{p}>0$ such that $\operatorname{dim}\left(\operatorname{ker} d\left(\eta_{t \xi}\right)_{p}\right)=0$ for every $t \in\left[-\epsilon_{p}, r_{c, b}^{-}+\epsilon_{p}\right] \backslash r_{c, b}^{-}$. As the initial conditions of the equations that define the $f^{-1}(c)$-Jacobi fields vary continuously with $p \in f^{-1}(c)$, one can define $\epsilon_{p}$ continuously depending on $p$. So for every $x \in f^{-1}(c)$ there exist open neighborhood $U$ of $x$ in $f^{-1}(c)$ and $\epsilon>0$ such that

$$
\operatorname{dim}\left(\operatorname{ker} d\left(\eta_{t \xi}\right)_{q}\right)=0
$$

for every $q \in U$ and $t \in\left[-\epsilon, r_{c, b}^{-}+\epsilon\right] \backslash r_{c, b}^{-}$. It also means that the maps $d\left(\eta_{t \xi}\right)_{q}$ are injective and consequently isomorphisms. As a result $\eta_{t \xi}$ is locally diffeomorphism. Now the fact that $\eta_{t \xi}$ 
is a diffeomorphism between the regular leaves implies that $\gamma_{x}\left(r_{c, b}^{-}\right)$is the only possibility to be a $f^{-1}(c)$-focal point (see Picture 3.11). Now on the one hand from the linear algebra we know that

$$
\operatorname{dim} \operatorname{ker} d\left(\eta_{r_{c, \xi}^{-}}\right)_{p} \geq \operatorname{dim} \operatorname{ker} d\left(\eta_{r_{c, \xi}^{-} \xi}\right)_{x}
$$

for every $x \in f^{-1}(c)$. On the other hand by Morse Index

$$
m\left(\gamma_{p}\right) \leq m\left(\gamma_{x}\right)
$$

for every $x \in f^{-1}(c)$. Therefore for every $x \in U \subset f^{-1}(c)$,

$$
m\left(\gamma_{x}\right)=m\left(\gamma_{p}\right)=\operatorname{dim} \operatorname{ker} d\left(\eta_{r_{c, b}^{-} \xi}\right)_{p}=k
$$

Note that the domain of $\gamma_{x}$ is as $I=\left[-\epsilon, r_{c, b}^{-}+\epsilon\right]$. As a result $d\left(\eta_{r_{c, b}^{-} \xi}\right)$ has constant rank.

To terminate the proof of item $(a)$, we recall the definition of metric $\tilde{F}$ given in 1.5.1. According to Lemma 1.5.1, $(M, \tilde{F})$ is a Finsler space. Let $\gamma$ be the unit speed minimizing geodesic discussed in the expression of Proposition 3.2.1. That is in the regular part we have

$$
\gamma^{\prime}(t)=\frac{\nabla f(\gamma)}{F(\nabla f)}=\xi
$$

Then according to Lemma 1.5.4, $\tilde{\gamma}(t)=\gamma(-t)$ is a $\tilde{F}$-geodesic. Notice that $\tilde{\gamma}$ and $\gamma$ have the same trace with different directions and by Lemma 1.5.4, both are unit speed geodesics. Actually if $\gamma:[0, l] \longrightarrow M$, then $\tilde{\gamma}:[-l, 0] \longrightarrow M$. So $\tilde{\gamma}$ departs from $f^{-1}(b)$ and meets all of the leaves exactly once and then reaches to $f^{-1}(a)$. By Lemma 1.5.5, $\tilde{\gamma}$ intersects each leaf $\tilde{F}$-orthogonally. Define $\tilde{\eta}_{r_{c, a}^{-}(-\xi)}: f^{-1}(c) \longrightarrow f^{-1}(a), x \mapsto \gamma\left(-r_{c, a}^{-}\right)=\tilde{\gamma}\left(r_{c, a}^{-}\right)$, where

$$
\gamma(t)=\exp _{x}(t \xi)
$$

and note that by Proposition 3.2.1, this map is well-defined.

Lemma 3.5.6. The derivative of map $\tilde{\eta}_{r_{c, a}^{-} \xi}: f^{-1}(c) \longrightarrow f^{-1}(a)$ has constant rank.

Proof. Define $\tilde{\eta}_{r(-\xi)}: f^{-1}(d) \longrightarrow f^{-1}(c), x \mapsto \gamma(-r)=\tilde{\gamma}(r)$, where

$$
\gamma(t)=\exp _{x}(t \xi)
$$

and $r=r_{c, b}^{-}-r_{d, b}^{-}$. Note that by Proposition 3.2.1, this map is well-defined. Comparing $\tilde{\eta}_{r(-\xi)}$ with $\eta_{-r \xi}^{-}$in Lemma 3.5.2, we have that indeed

$$
\tilde{\eta}_{r(-\xi)}=\eta_{-r \xi}^{-}
$$

and consequently $\tilde{\eta}_{r(-\xi)}$ is a diffeomorphism. Also by the same argument given for Lemma 3.5.4, mutatis mutandis, the derivative of the map $\tilde{\eta}_{r_{c, a}^{-}(-\xi)}: f^{-1}(c) \longrightarrow f^{-1}(a), x \mapsto \gamma\left(-r_{c, a}^{-}\right)=\tilde{\gamma}\left(r_{c, a}^{-}\right)$, has constant rank. Indeed one can prove that

$$
\gamma\left(-r_{c, a}^{-}\right)
$$


is a $f^{-1}(c)$-focal point of multiplicity $k$ if and only if $x$ is a critical point of $\tilde{\eta}_{r_{c, a}^{-}(-\xi)}$ and $\operatorname{dim} \operatorname{ker} d\left(\tilde{\eta}_{r_{c, a}^{-}(-\xi)}\right)_{x}=$ $k$. In fact in the case of being a focal point we have

$$
J\left(r_{c, a}^{-}\right)=\tilde{J}\left(-r_{c, a}^{-}\right)=0
$$

where $\tilde{J}$ and $J$ are the Jacobi fields with respect to $\tilde{F}$ along $\tilde{\gamma}$ and with respect to $F$ along $\gamma$, resp., whose relation is given in Lemma 1.5.8 (see Picture 3.11). This closes the proof of Lemma 3.5.6.

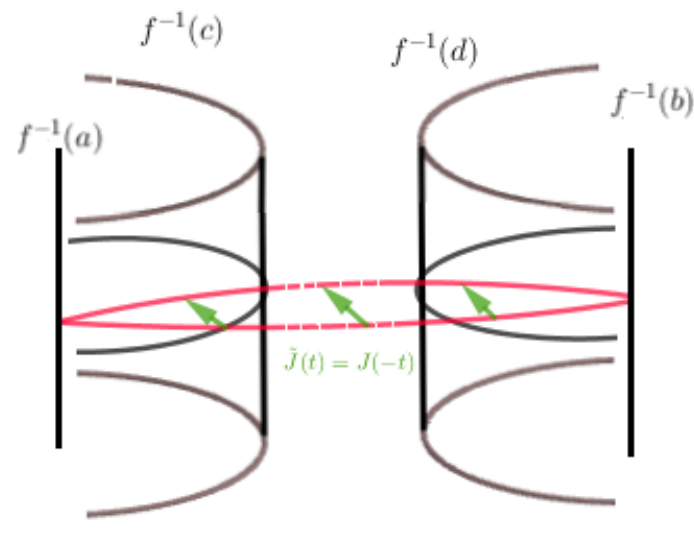

Figure 3.11

From Lemmas 3.5.4 and 3.5.6, one concludes that each regular level set $f^{-1}(c)$ is equifocal which complete the proof of item $(a)$.

Proof of item (b).

As a result of Lemmas 3.5.4 and 3.5.6, one can apply the constant rank theorem to show that $f^{-1}(b)$ and $f^{-1}(b)$ are immersed submanifolds with possible self-intersections. Let's prove that they are embedded, indeed.

Indeed, to prove that $f^{-1}(b)$ is embedded, in the case that $f^{-1}(b)$ has codimension one. Choose $c$ so close to $b$. From Proposition 3.2.1 we know that $f^{-1}(c) \| f^{-1}(b)$ which means

$$
f^{-1}(c) \subset C_{r_{c, b}^{-}}^{-}\left(f^{-1}(b)\right), \text { where } r_{c, b}^{-}=d_{F}\left(f^{-1}(c), f^{-1}(b)\right) .
$$

Let $C^{-}$be the connected component of $C_{r_{c, b}^{-}}^{-}\left(f^{-1}(b)\right)$ containing $f^{-1}(c)$. As $C^{-}$is connected, $f^{-1}(c)$ is closed, and $\operatorname{dim} C^{-}=\operatorname{dim} f^{-1}(c)$ we conclude that $C^{-}=f^{-1}(c)$ which means $f^{-1}(b)$ must be embedded. Because otherwise $C^{-}$must have self-intersections.

Now to show that $f^{-1}(a)$, in the case that it has codimension 1, as by Proposition 3.2.1, $f^{-1}(a) \| f^{-1}(c)$ we have that

$$
f^{-1}(c) \subset C_{r_{c, a}^{+}}^{+}\left(f^{-1}(a)\right) \text { where } r_{c, a}^{+}=d_{F}\left(f^{-1}(a), f^{-1}(c)\right) .
$$

Finally by following the argument provided for $f^{-1}(b)$, mutatis mutandis, one can show that $f^{-1}(a)$ is an embedded submanifold.

If $f^{-1}(b)$ has codimension greater than 1 . In this case the same proof as Lemma 3.3.2 can be applied. 


\subsubsection{The distance between the leaves}

Finally as it was promised we provide a formula that together with the formula given in Proposition 3.2.1 one will be able to calculate the distances between two leaves corresponding to a given singular Finsler foliation. Moreover we show that given a Finsler foliation, the inner product of $\nabla f$ and $\xi \in \mathfrak{C}^{1}\left(f^{-1}(c)\right)$ is constant along each regular leaf $f^{-1}(c)$. Let's first define the angle between two vectors in a Finsler space and then the mentioned result. Observe that there are several definitions for the angle in the case of Finsler space and with respect to the application one can choose which definition is more appropriate. We provide two definitions as follows.

Definition 3.5.7. Given a Finsler space $(M, F)$ and two vectors $v, w \in T M$, then one can define the angle from $v$ to $w$ using the formula

$$
\cos \theta(v, w)=\frac{g_{v}(v, w)}{F(v) \sqrt{g_{v}(w, w)}} .
$$

Definition 3.5.8. Given a Finsler space $(M, F)$ and two vectors $v, w \in T M$, then one can define the angle from $v$ to $w$ using the formula

$$
\cos \tilde{\theta}(v, w)=\frac{g_{v}(v, w)}{F(v) F(w)}
$$

Observe that in neither of these two definitions the angle is not symmetric. That is

$$
\cos \theta(v, w) \neq \cos \theta(w, v) \text { and } \cos \tilde{\theta}(v, w) \neq \cos \tilde{\theta}(w, v)
$$

or in other words, the angle from $v$ to $w$ is not equal the angle from $w$ to $v$.

Remark 3.5.9. Given a smooth function $f:(M, F) \longrightarrow \mathbb{R}$, according to these two definitions, one can write

$$
\cos \theta(\nabla f, \xi)_{p}=\frac{g_{\nabla f}(\nabla f, \xi)}{F(\nabla f) \sqrt{g_{\nabla f}(\xi, \xi)}}(p),
$$

and

$$
\cos \tilde{\theta}(\nabla f, \xi)_{p}=\frac{g_{\nabla f}(\nabla f, \xi)}{F(\nabla f)}(p)
$$

where $\xi \in \mathfrak{C}^{-1}\left(f^{-1}(c)\right)$ which is on the opposite side of $\nabla f$ with respect to the hyperplane $T_{\pi(\xi)} f^{-1}(c)$ and $\theta(\nabla f, \xi)=\varangle(\nabla f, \xi)$, for the regular value $c$.

Note that generally the inner product between the vectors orthogonal to a regular leaf at each point, depends on that point. However in the case in which we have a Finsler foliation, we will see that the inner product between these vectors with respect to the metric $g_{\nabla f}$ is constant along that leaf. Moreover the angle between two vectors orthogonal to a regular leaf is not necessarily constant along that leaf. However we will see that given a Finsler foliation, if one uses Definition 3.5.8, the angle remains constant along each regular leaf.

Proposition 3.5.10. Let $f: M \longrightarrow[a, b]$ be a F-transnormal function with $F^{2}(\nabla f)=\mathfrak{b}(f)$ such that for every $c$ and $d \in[a, b], f^{-1}(c)$ is equidistant to $f^{-1}(d)$ and also each $f^{-1}(c)$ is connected. Suppose that there is not any critical point in $(a, b)$. Let $\tilde{F}(y):=F(-y)$, for $y \in T_{\pi(y)} M$. Then 


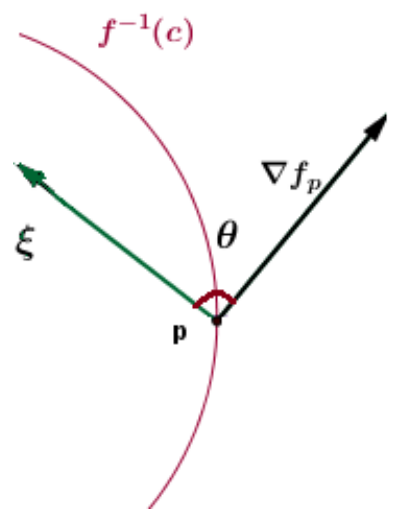

Figure 3.12

a. $f$ is $\tilde{F}$-transnormal with $\tilde{\mathfrak{b}}(f)=\mathfrak{b}(f)\left(g_{\frac{\nabla f}{F(\nabla f)}}\left(\frac{\nabla f}{F(\nabla f)}, \xi\right)\right)^{2}$, where $\xi \in \mathfrak{C}^{1}\left(f^{-1}(c)\right)$ which is on the opposite side of $\nabla f$ with respect to the hyperplane $T_{\pi(\xi)} f^{-1}(c)$, for the regular value $c$;

b. for every $c<d \in[a, b]$ and for every $q \in f^{-1}(c)$,

$$
d_{F}\left(f^{-1}(d), q\right)=d_{F}\left(f^{-1}(d), f^{-1}(c)\right)=\int_{c}^{d} \frac{d s}{\sqrt{\tilde{\mathfrak{b}}(s)}}=L(\tilde{\alpha}),
$$

where $\tilde{\alpha}$ is a reparametrization of the integral curve of $\tilde{\nabla} f$;

In the case that $f^{-1}(a)$ and $f^{-1}(b)$ are singular leaves of codimension 1 ,

c. there exist $r_{c, a}^{ \pm}, r_{c, b}^{ \pm}>0$ such that $f^{-1}(c)=\tilde{C}_{r_{c, a}^{ \pm}}^{ \pm}\left(f^{-1}(a)\right)=\tilde{C}_{r_{c, b}^{ \pm}}^{ \pm}\left(f^{-1}(b)\right)$, for every $c \in(a, b)$. In particular,

(-) for every $c, d \in[a, b], f^{-1}(c)$ is equidistant to $f^{-1}(d)$ with respect to $\tilde{F}$, i.e. $f^{-1}(c) \| f^{-1}(d)$ and $f^{-1}(d) \| f^{-1}(c)$ with respect to $\tilde{F}$.

Proof. First note that by Lemma 1.5.1, $(M, \tilde{F})$ is a Finsler space. Let us show that $f$ is $\tilde{F}$ transnormal. Observe that since by hypothesis, for every $c \in[a, b], f^{-1}(c)$ is equidistant to $f^{-1}(b)$, one can say that for every $p \in f^{-1}(c)$ there exists a unitary speed geodesic $\gamma$ with

$$
d_{F}\left(f^{-1}(b), f^{-1}(c)\right)=d_{F}\left(f^{-1}(b), p\right)=L\left(\left.\gamma\right|_{\left[0, r_{c}\right]}\right)=r_{c} .
$$

Since this geodesic also minimizes the distance from $f^{-1}(b)$ to $f^{-1}(c)$, then it is orthogonal to both these submanifolds. So one can define a vector field $\xi$ on $M$ in such a way that at each point $p$ we have

$$
\xi(p)=\xi\left(\gamma\left(r_{c}\right)\right)=\gamma^{\prime}\left(r_{c}\right)
$$

Indeed $\xi(p) \in \mathfrak{C}_{p}^{1}\left(f^{-1}(c)\right)$ and notice that, as the geodesic $\gamma$ is minimizing the distance between the leaves, it is on the opposite side of $\nabla f$ with respect to the hyperplane $T_{p} f^{-1}(c)$. Define $t \longrightarrow$ $\tilde{\gamma}(t):=\gamma(-t)$. According to Lemma 1.5.4, $\tilde{\gamma}$ is a geodesic with respect to $\tilde{F}$ and it is orthogonal to both $f^{-1}(c)$ and $f^{-1}(b)$. Put $\tilde{\xi}=-\xi$ and note that

$$
\tilde{\xi}(\tilde{\gamma}(t))=\tilde{\xi}(\gamma(-t))=-\xi(\gamma(-t))=-\gamma^{\prime}(-t)=\tilde{\gamma}^{\prime}(t)
$$


We are about to calculate $\tilde{F}^{2}(\tilde{\nabla} f)$, that is the norm gradient of $f$ with respect to $\tilde{F}$. Observe that

$$
\tilde{\nabla} f \in \tilde{\mathfrak{C}}\left(f^{-1}(c)\right)
$$

where $\tilde{\mathfrak{C}}$ is the orthogonal cone with respect to $\tilde{F}$. Furthermore as $f^{-1}(c)$ is a hypersurface, $\tilde{\nabla} f$ is in the same side of $\tilde{\xi}$ or in the opposite side of this with respect to the hyperplane $T_{\tilde{\gamma}} f^{-1}(c)$. Since with respect to Comment 3.2.3, $f$ must be increasing in the direction of $\tilde{\nabla} f, \tilde{\nabla} f$ is in the same side of $\tilde{\xi}$ with respect to the hyperplane $T_{\tilde{\gamma}} f^{-1}(c)$. In other words,

$$
\tilde{\nabla} f=\lambda \tilde{\xi}
$$

where $\lambda: M \longrightarrow \mathbb{R} \geq 0$ which is strictly positive in regular points. See Picture 3.13 below. Let us

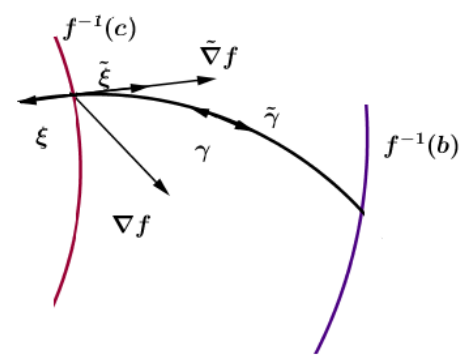

Figure 3.13

find $\tilde{F}^{2}(\tilde{\nabla} f)$. First observe that by positive homogeneity of $\tilde{F}$ we have

$$
\tilde{F}^{2}(\tilde{\nabla} f)=\tilde{F}^{2}(\lambda \tilde{\xi})=\lambda^{2} F^{2}(\xi)=\lambda^{2} .
$$

Moreover

$$
\tilde{F}^{2}(\tilde{\nabla} f)=g_{\tilde{\nabla} f}(\tilde{\nabla} f, \tilde{\nabla} f)=d f(\tilde{\nabla} f)=\lambda d f(\tilde{\xi})=-\lambda d f(\xi) .
$$

From EQs. 3.27 and 3.28 we have

$$
\tilde{F}(\tilde{\nabla} f)=-d f(\xi)
$$

Claim 3.5.11. Let $f^{o}=\left.f\right|_{f^{-1}(a, b)}$ and $M^{o}=f^{-1}(a, b)$. Given a regular point $p \in M^{o}$, consider $d f_{p}^{o}: T_{p} M^{o} \longrightarrow T_{f^{o}(p)}(a, b)$. Then for every $w \in T_{f^{o}(p)}(a, b)$ there exists a unique $\mu_{w} \in \mathbb{R}$ such that $d f_{p}^{o}\left(\mu_{w} \xi\right)=w$.

Proof. Note that for the map $d f_{p}^{o}: T_{p} M^{o} \longrightarrow T_{f^{o}(p)}(a, b)$, as $p$ is not a critical point we have that

$$
d f_{p}^{o}(\xi) \neq 0
$$

It means $d f_{p}$ is a surjective map and so one can write $d f_{p}^{o}(\xi)=\frac{1}{\mu_{w}} w$ for some $\mu_{w} \in \mathbb{R}$. Also if for some $\mu_{w}$ and $\bar{\mu}_{w}$ we have $d f_{p}^{o}\left(\mu_{w} \xi\right)=d f_{p}^{o}\left(\bar{\mu}_{w} \xi\right)=w$, then by linearity of $d f_{p}^{o}$ we must have $\mu_{w}=\bar{\mu}_{w}$, which concludes the unicity.

Lemma 3.5.12. $f^{o}:\left(M^{o}, g_{\xi}\right) \longrightarrow\left((a, b),\|\cdot\|_{2}\right)$, in which $\|w\|_{2}=\left|\mu_{w}\right|$, is a Riemannian submersion. 
Proof. As it was discussed in the proof of Claim 3.5.11, given any regular point $p \in M^{o}, d f_{p}^{o}$ is a surjective map and so we have a submersion. Observe that indeed $\left\{d f_{p}^{o}(\xi)\right\}$ is a basis of $T_{f^{o}(p)}(a, b)$. Now consider $\left.d f_{p}^{o}\right|_{\mathcal{H}_{p}}:\left(\mathcal{H}_{p}, g_{\xi}\right) \longrightarrow\left(T_{c}(a, b),|| \cdot \|_{2}\right)$, where $f^{o}(p)=c$ and

$$
\mathcal{H}_{p} \oplus T_{p} f^{-1}(c)=T_{p} M^{0}
$$

is the decomposition associated to the Riemannian metric $g_{\xi}$. Observe that for every $w \in T_{c}(a, b)$ there exists some $v \in \mathcal{H}_{p}$ such that $d f_{p}^{o}(v)=w$ and also, according to Claim 3.5.11, there exists a unique $\mu_{w} \in \mathbb{R}$ such that $w=d f_{p}^{o}\left(\mu_{w} \xi\right)$. As such a $v$ can be written uniquely as a multiplication of $\xi$, from above discussion we must have $v=\mu_{w} \xi$. It guarantees that $\left.d f_{p}^{o}\right|_{\mathcal{H}_{p}}$ is injective and so we have a linear isomorphism. We shall show that $\left.d f_{p}^{o}\right|_{\mathcal{H}_{p}}$ is an isometry. For every $v \in \mathcal{H}_{p}$,

$$
\|v\|=\sqrt{g_{\xi}(v, v)}=\sqrt{g_{\xi}\left(\mu_{w} \xi, \mu_{w} \xi\right)}=\left|\mu_{w}\right|
$$

Next by hypothesis,

$$
\left\|d f_{p}^{o}(v)\right\|_{2}=\left\|d f_{p}^{o}\left(\mu_{w} \xi\right)\right\|_{2}=\left|\mu_{w}\right| .
$$

Comparing EQs. 3.30 and 3.31, we conclude that $d f_{p}^{o}$ is an isometry and so we have a Riemannian submersion.

Claim 3.5.13. $\xi$ is a projectable vector field.

Proof. Define $T: f^{-1}(c) \longrightarrow T_{c}((a, b)), p \mapsto T(p):=d f_{p}(\xi)$. With respect to the points that $\xi$ at each point is an orthogonal unitary vector and $\left.f\right|_{M^{0}}$ is a Riemannian submersion, we deduce that

$$
\|T(p)\|_{2}=1
$$

for every $p \in f^{-1}(c)$. Thus $T$ in fact takes values in $\{-1,1\} \subset T_{c}((a, b))$. As $f^{-1}(c)$ is connected and $T$ is continuous, we conclude that $T$ is constant (which in our case because of the direction of $\gamma, T \equiv-1)$. I.e. $\xi$ is projectable and so $d f(\xi)=\bar{\xi}(f)$ where $\bar{\xi} \in \mathfrak{X}((a, b))$ (indeed $\bar{\xi}$ is the constant vector -1$)$.

Now from EQ. 3.29 and Claim 3.5.13, one has $\tilde{F}(\tilde{\nabla} f)=-d f(\xi)=-\bar{\xi}(f)$ with which we conclude that $\tilde{F}(\tilde{\nabla} f)$ depends only on $f$ and it is constant along each leaf. Finally as a consequence of above discussion and EQ. 3.28, one can write

$$
\tilde{F}^{2}(\tilde{\nabla} f)=\tilde{\mathfrak{b}} \circ f
$$

where $\tilde{\mathfrak{b}} \circ f=(d f(\xi))^{2}=\left(g_{\nabla f}(\nabla f, \xi)\right)^{2}$, which means $f$ is $\tilde{F}$-transnormal. Finally to find the formula in item $(a)$, we have

$$
\tilde{\mathfrak{b}}(f)=\left(g_{\nabla f}(\nabla f, \xi)\right)^{2}=F^{2}(\nabla f)\left(g_{\frac{\nabla f}{F(\nabla f)}}\left(\frac{\nabla f}{F(\nabla f)}, \xi\right)\right)^{2}=\mathfrak{b}(f)\left(g_{\frac{\nabla f}{F(\nabla f)}}\left(\frac{\nabla f}{F(\nabla f)}, \xi\right)\right)^{2} .
$$

Let us prove item $(b)$. The fact that $f$ is $\tilde{F}$-transnormal with $\tilde{\mathfrak{b}}(f)=\left(g_{\nabla f}(\nabla f, \xi)\right)^{2}$ together 
with Proposition 3.2.1 guarantee that for every $q \in f^{-1}(c)$,

$$
d_{\tilde{F}}\left(q, f^{-1}(d)\right)=d_{\tilde{F}}\left(f^{-1}(c), f^{-1}(d)\right)=\int_{c}^{d} \frac{d s}{\sqrt{\tilde{\mathfrak{b}}(s)}}=L(\tilde{\alpha}),
$$

where $\tilde{\alpha}$ is the reparametrization of the integral curve of $\tilde{\nabla} f$ as $\tilde{\alpha}\left(\frac{t}{\tilde{F}(\tilde{\nabla} f)}\right)$.

Claim 3.5.14. For every $q \in f^{-1}(c)$,

$$
d_{\tilde{F}}\left(q, f^{-1}(d)\right)=d_{F}\left(f^{-1}(d), q\right) .
$$

Proof. Given $q \in f^{-1}(c)$, let $\tilde{\gamma}$ be the unitary speed geodesic such that

$$
d_{\tilde{F}}\left(q, f^{-1}(d)\right)=L\left(\left.\tilde{\gamma}\right|_{[0, r]}\right)=r
$$

Then by Lemma 1.5.4 and the relation $\tilde{F}\left(\tilde{\gamma}^{\prime}\right)=F\left(-\tilde{\gamma}^{\prime}\right)=F\left(\gamma^{\prime}\right)=1, \gamma(t):=\tilde{\gamma}(-t)$ is a unitary speed geodesic with $\gamma(-r)=\tilde{\gamma}(r) \in f^{-1}(d)$ and $\gamma(0)=\tilde{\gamma}(0)=q$. See Picture 3.14. Hence $d_{F}\left(f^{-1}(d), q\right) \leq r$. Suppose that $\beta$ is a unitary speed geodesic with

$$
d_{F}\left(f^{-1}(d), q\right)=L\left(\left.\beta\right|_{[o, s]}\right)=s<r .
$$

Then by Lemma 1.5.4, $\tilde{\beta}(t):=\beta(-t)$ is a geodesic such that $\tilde{\beta}(0) \in f^{-1}(d)$ and $\tilde{\beta}(-s)=q$ that means $d_{\tilde{F}}\left(q, f^{-1}(d)\right)<r$ which is a contradiction and so

$$
d_{F}\left(f^{-1}(d), q\right)=r=d_{\tilde{F}}\left(q, f^{-1}(d)\right) .
$$
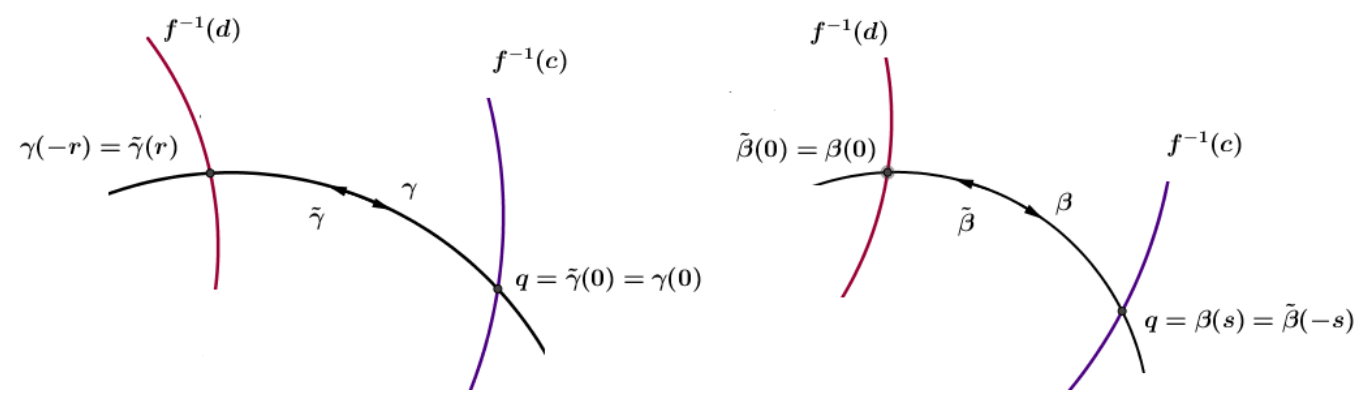

Figure 3.14

To conclude the proof, by Claim 3.5.14 and EQ. 3.32 we have that for every $q \in f^{-1}(c)$,

$$
d_{F}\left(f^{-1}(d), q\right)=\int_{c}^{d} \frac{d s}{\sqrt{\tilde{\mathfrak{b}}(s)}}=L(\tilde{\alpha})
$$


and because we have the same integral for every $q$, one cam get the following equality

$$
d_{F}\left(f^{-1}(d), q\right)=d_{F}\left(f^{-1}(d), f^{-1}(c)\right)=\int_{c}^{d} \frac{d s}{\sqrt{\tilde{\mathfrak{b}}(s)}}=L(\tilde{\alpha}) .
$$

To prove item $(c)$, with respect to the fact that $f$ is $\tilde{F}$-transnormal, Theorem 3.3.1, and Lemma 1.5.6 we have this item proved.

Corollary 3.5.15. Let $f:(M, F) \longrightarrow[a, b]$ be a F-transnormal function for which $\mathcal{F}=\left\{f^{-1}(c)\right\}_{c \in[a, b]}$ is a singular Finsler foliation. Then

$$
g_{\nabla f}(\nabla f, \xi)=\text { constant }
$$

along each regular leaf $f^{-1}(c)$, where $\xi$ is an orthogonal unitary vector field along the regular leaves which is on the opposite side of $\nabla f$ with respect to the hyperplane $T_{\pi(\xi)} f^{-1}(c)$.

Moreover,

$$
\cos \tilde{\theta}(\nabla f, \xi)(f)=\frac{d f(\xi)}{F(\nabla f)}
$$

That is, considering EQ. 3.26, the angle between $\nabla f$ and $\xi$ is constant along each leaf.

$\star$ In particular if the angle $\tilde{\theta}$ is not constant along each leaf, then the foliation cannot be Finslerian.

Proof.

By Claim 3.5.13, $\xi$ is a projectable vector field and so

$$
g_{\nabla f}(\nabla f, \xi)=d f(\xi)=\text { constant }
$$

along each regular leaf.

For the next assertion, with respect to Lemma 3.5.15 and the point that $f$ is $F$-transnormal function, the proof is at hand.

Example 3.5.16. We recall Example 2.4.7. In this example we have a foliation $\mathcal{F}=\left\{\Sigma_{r}^{h}\right\}_{r>0}$ with $f(x)=\|x\|^{2}$ and $F^{2}(\nabla f)=4 f$. First of all, at each $x \in f^{-1}(c)$, the vectors $\frac{x}{\|x\|}$ and $\frac{-x}{\|x\|}$ are orthogonal to $f^{-1}(c)$ with respect to the metric $h$. So by Corollary 2.2.5 they are unitary vectors and so note that by Corollary 2.2.7,

$$
\frac{x}{\|x\|}+W(x) \text { and } \frac{-x}{\|x\|}+W(x)
$$

are orthogonal to $f^{-1}(c)$ with respect to $F$. By considering the directions of these two vectors according to plane $T_{x} f^{-1}(c)$, we have that $\frac{x}{\|x\|}+W(x)$ is a multiplication of $\nabla f$ and also

$$
-\frac{x}{\|x\|}+W(x)=\xi
$$

Let us calculate the inner product between $\xi$ and $\nabla f$ with respect to the Riemannian metric $g_{\nabla f}$.

$$
g_{\nabla f}(\nabla f, \xi)=g_{\nabla f}\left(\nabla f, \frac{-x}{\|x\|}+W(x)\right)=-d f\left(\frac{x}{\|x\|}\right)=-2 \sqrt{f},
$$


which means this inner product is constant along each leaf as it was expected by Corollary 3.5.15. Here we used the point that $W$ is tangent to each leaf and so

$$
g_{\nabla f}(\nabla f, W(x))=0 .
$$

Now let us, using formula 3.26, calculate the angle from $\nabla f$ to $\xi$. According to above calculations one gets

$$
\cos \theta=\frac{g_{\nabla f}(\nabla f, \xi)}{F(\nabla f)}=\frac{-2 \sqrt{f}}{2 \sqrt{f}}=-1 .
$$

Corollary 3.5.17. Note that in Proposition 3.5.10 one can also say that $f$ is $\tilde{F}$-transnormal with

$$
\tilde{\mathfrak{b}}(f)=\mathfrak{b}(f) g_{\zeta}^{2}(\zeta, \xi)
$$

where $\xi, \zeta \in \mathfrak{C}^{1}\left(f^{-1}(c)\right)$ are, resp., in the opposite side and the same side of $\nabla f$ with respect to the hyperplane $T_{\pi(\xi)} f^{-1}(c)$ for every regular value $c$.

In particular the inner product of two vectors $\xi$ and $\zeta$ with respect to metric $g_{\zeta}$ is constant along each regular leaf.

Proof. According to item (a) of Proposition 3.5.10 one can write

$$
\begin{aligned}
\tilde{\mathfrak{b}}(f) & =\mathfrak{b}(f)\left(g_{\frac{\nabla f}{F(\nabla f)}}\left(\frac{\nabla f}{F(\nabla f)}, \xi\right)\right)^{2} \\
& =\mathfrak{b}(f)\left(g_{\zeta}(\zeta, \xi)\right)^{2},
\end{aligned}
$$

where $\zeta:=\frac{\nabla f}{F(\nabla f)}$. Observe that as it is mentioned in the expression of Proposition 3.5.10, $\nabla f$ and $\xi$ are in the different side of the hyperplane $T_{\pi(\xi)} f^{-1}(c)$ for every regular value $c$.

Lemma 3.5.18. Let $f:(M, F) \longrightarrow[a, b]$ be a F-transnormal function for which $\mathcal{F}=\left\{f^{-1}(c)\right\}_{c \in[a, b]}$ is a singular Finsler foliation and $\theta$ be the angle between $\nabla f$ and $\xi$. Then $\theta$ is constant along each regular leaf $f^{-1}(c)$, if and only if Hess $F(\xi, \xi)$ is constant along $f^{-1}(c)$. Here $\xi \in \mathfrak{C}\left(f^{-1}(c)\right)$ which is on the opposite side of $\nabla f$ with respect to the hyperplane $T_{\pi(\xi)} f^{-1}(c)$.

Proof. According to Equation 3.25 one can write

$$
\cos \theta=\frac{g_{\nabla f}(\nabla f, \xi)}{F(\nabla f) \sqrt{g_{\nabla f}(\xi, \xi)}}
$$

In Lemma 3.5.15 we prove that $g_{\nabla f}(\nabla f, \xi)$ is constant along each leaf. So observe that we have

$$
g_{\nabla f}(\nabla f, \xi)=\left.\frac{1}{2} \frac{\partial F^{2}}{\partial s}(\nabla f+s \xi)\right|_{s=0}=F(\nabla f) d F_{\nabla f} \xi
$$

which implies that $d F(\nabla f) \xi$ is constant along each leaf. Moreover,

$$
\begin{aligned}
g_{\nabla f}(\xi, \xi) & =\left.\frac{1}{2} \frac{\partial^{2} F^{2}}{\partial s \partial t}(\nabla f+s \xi+t \xi)\right|_{s=t=0} \\
& =\left.\frac{\partial}{\partial s}\left[\left.F(\nabla f+s \xi) \frac{\partial^{2} F}{\partial s \partial t}(\nabla f+s \xi+t \xi)\right|_{t=0}\right]\right|_{s=0} \\
& =\left(d F_{\nabla f} \xi\right)^{2}+F(\nabla f) \operatorname{Hess} F(\xi, \xi),
\end{aligned}
$$


which concludes that, along each leaf, $g_{\nabla f}(\xi, \xi)$ is constant if and only if $\operatorname{Hess} F(\xi, \xi)$ is constant. It completes the proof.

Let us close this part with a Lemma.

Lemma 3.5.19. Let $f:(M, F) \longrightarrow[a, b]$ be a F-transnormal function such that there is not any critical values in the interval $(a, b)$. Then $f:\left(f^{-1}(a, b), g_{\nabla f}\right) \longrightarrow\left((a, b), g_{2}\right)$, where $g_{2}=\frac{1}{\mathfrak{b}} d x^{2}$, is a Riemannian submersion. Also $\mathcal{F}=\left\{f^{-1}(c)\right\}_{c \in(a, b)}$ is a Riemannian foliation with metric $g_{\nabla f}$.

Proof. Let $f^{-1}(a, b)=M^{o}$ and note that for the map $d f_{p}: T_{p} M^{o} \longrightarrow T_{f(p)}(a, b)$ we have that

$$
d f_{p}(\nabla f)=F^{2}(\nabla f) \neq 0
$$

for every $p \in M^{o}$. It means $d f_{p}$ is a surjective map and so we have a submersion. Now consider $\left.d f_{p}\right|_{\mathcal{H}_{p}}:\left(\mathcal{H}_{p}, g_{\nabla f}\right) \longrightarrow\left(T_{c}(a, b), g_{2}\right)$, where $f(p)=c$ and

$$
\mathcal{H}_{p} \oplus T_{p} f^{-1}(c)=T_{p} M^{o}
$$

is the decomposition associated to the Riemannian metric $g_{\nabla f}$. We shall show that $\left.d f_{p}\right|_{\mathcal{H}_{p}}$ is an isometry. Note that $\left\{d f_{p}(\nabla f)\right\}$ is the basis of $T_{p} M^{o}$ and so each $w \in T_{P} M^{o}$ can be written in a unique way as $w=\mu_{w} . d f_{p}(\nabla f)$. Observe that also for every $v \in \mathcal{H}_{p}, v=\mu_{v} \nabla f(p)$ for some unique $\mu_{v} \in \mathbb{R}$. From these facts and also the linearity of $d f_{p}$ we deduce the injectivity. It means we have a linear isomorphism. Now, first

$$
\begin{aligned}
\|v\|=\sqrt{g_{\nabla f}(v, v)}=\sqrt{g_{\nabla f}\left(\mu_{v} \nabla f, \mu_{v} \nabla f\right)} & =\left|\mu_{v}\right| \sqrt{g_{\nabla f}(\nabla f, \nabla f)} \\
& =\left|\mu_{v}\right| \sqrt{F^{2}(\nabla f)}=\left|\mu_{v}\right| \sqrt{\mathfrak{b}(f)},
\end{aligned}
$$

Next,

$$
\begin{aligned}
\left|d f_{p}(v)\right|=\sqrt{g_{2}\left(d f_{p}(v), d f_{p}(v)\right)} & =\left|\mu_{v}\right| \sqrt{g_{2}\left(d f_{p}(\nabla f), d f_{p}(\nabla f)\right)}=\left|\mu_{v}\right| \sqrt{g_{2}(\mathfrak{b}(f), \mathfrak{b}(f))} \\
& =\left|\mu_{v}\right| \sqrt{\frac{1}{\mathfrak{b}(f)}(\mathfrak{b}(f))^{2}}=\left|\mu_{v}\right| \sqrt{\mathfrak{b}(f)}
\end{aligned}
$$

Comparing EQs. 3.34 and 3.35 we conclude that $d f_{p}$ is an isometry and so we have a Riemannian submersion.

to prove the last assertion observe that by Proposition 1.3.31, the gradient of $f$ with respect to $g_{\nabla f}$ coincides with $\nabla f$ and both have the same norm. As $f$ is $F$-transnormal, by Remark 1.3.35, it is also $g_{\nabla f}$-transnormal on $M^{o}$. Therefore by Wang's Theorem,

$$
\mathcal{F}=\left\{f^{-1}(c)\right\}_{c \in(a, b)}
$$

is a Riemannian foliation. 


\section{Chapter 4}

\section{Applications}

\subsection{Introduction}

For the application part, two applications on the propagation of waves of fire and water are introduced. Indeed, applying the obtained results in the Finsler case together with some concepts in Physics, one can have some results related to the natural phenomena. For this purpose we first give some elementary concepts of Physics.

\subsection{Huygens's principle}

One can think of Huygens' principle as a mathematical principle rather than a principle of physics. Indeed the waves including sound, water, light, fire, electromagnetic waves, etc. obey the principle. So we consider briefly the fundamental notions of geometric optics and note that we will have the same concepts and facts for the behavior of the wave in other mentioned ambients. According to the extremal principle of Fermat [Arn13], light travels from a point $p$ to a point $q$ in the shortest possible time. The speed of the light can depend on both the point $q$ and on the direction of the ray. The characteristic of a medium can be described by giving the indicatrix in the tangent space at each point $q$.

Definition 4.2.1. Suppose that we are given a point $p$ which is also a source that emits waves. Let $t>0$ and look at the set of all points $q$ to which the wave from $p$ reaches after time $t$. Note that such a set consists of points to which wave can travel in time $t$ and no faster. The set $\{q\}$ is called the wavefront of the point $p$.

Note that in particular we have

$$
d(p,\{q\})=t .
$$

Also observe that this point can be replaced with a plane, a curve, etc. So, for example, for a sound wave spreading out from a point source we are faced with a spherical wavefront and a plane wavefront for a parallel beam of light, such as that emitted from a laser. Roughly speaking, the wavefront has different shapes depending on the nature of the source, the medium, and the obstructions encountered. See Picture 4.1.

Definition 4.2.2. Imagine that each point on the wavefront acts as a point source that emits spherical wavefronts. These wavefronts travel with the same velocity in the medium. At any later 


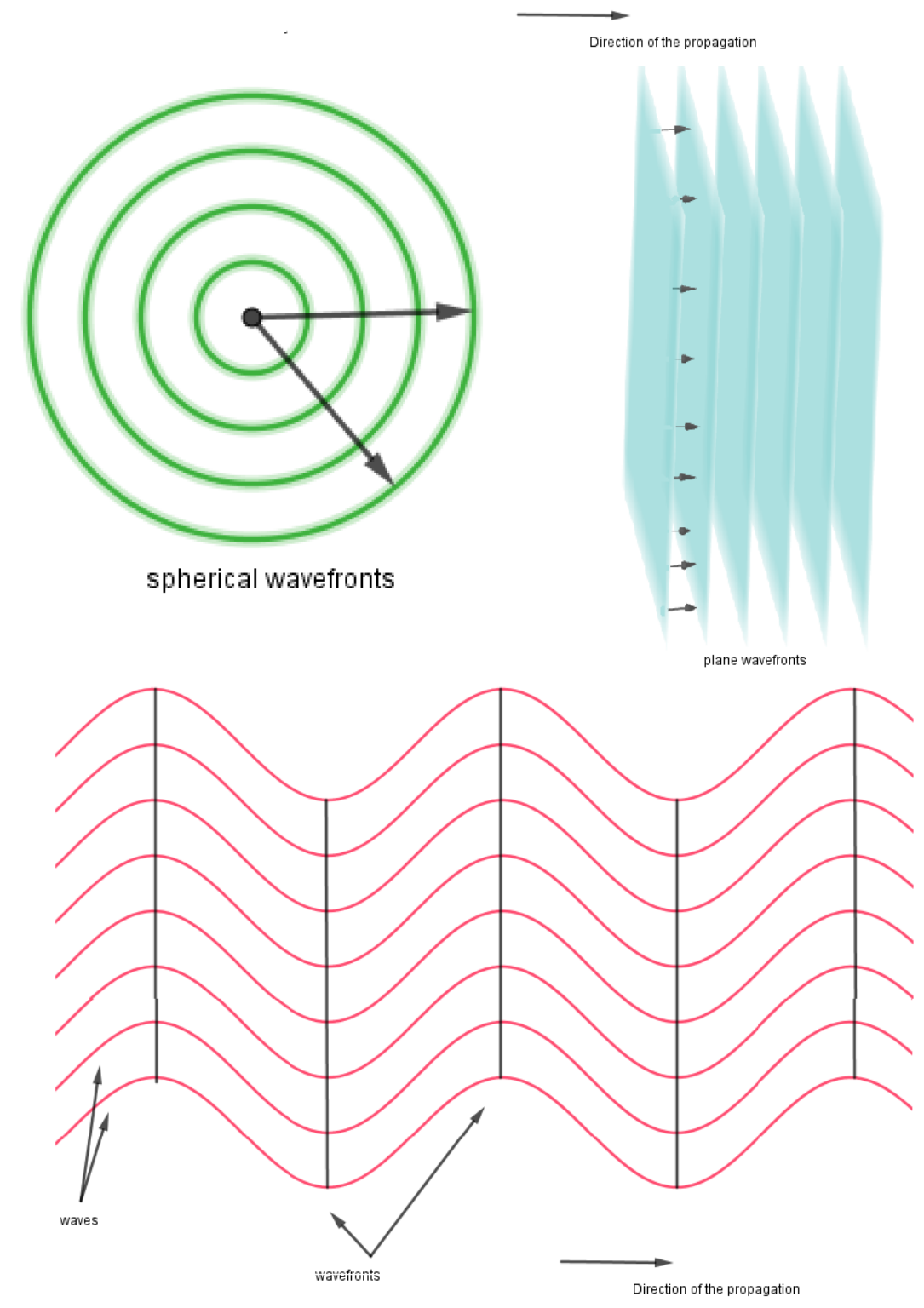

Figure 4.1

time, a surface tangent to each wavefront (at a single point) is called the envelope of the spherical wavefronts.

Observe that in particular we have

$$
d(\{q\}, \text { envelope })=c \Delta t,
$$

where $c \Delta t$ is the radius of each wavefront. See Picture 4.2 to see the wavefront and envelope in two special cases

Note that a wavefront could be the surface over which the wave has a maximum (the crest of a water wave, for example) or a minimum (the trough of the same wave) value. The shape of a wavefront is usually determined by the geometry of the source. A point source has wavefronts that are spheres whose centers are at the point source. A fluorescent tube would have wavefronts that are cylinders concentric with the tube itself. A very large sheet of material that is uniformly illuminated would generate wavefronts that are plane waves parallel to the sheet. The direction of 


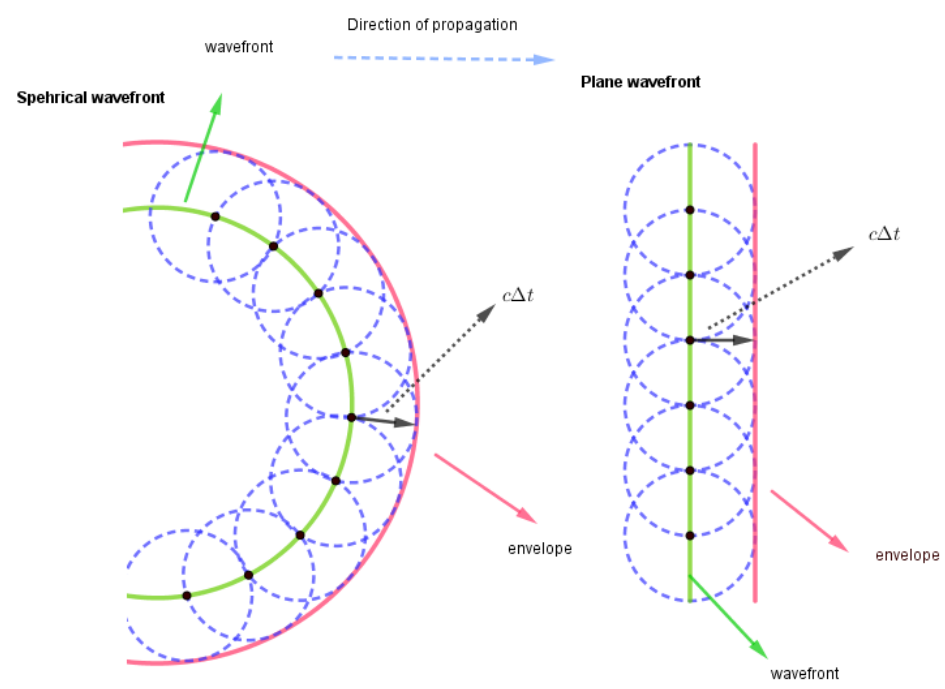

Figure 4.2

propagation of the wave is always perpendicular to the surface of the wavefront at each point. Thus, the wavefronts of a point source are spheres and the wave propagates radially outward the radius of a sphere is perpendicular to its circumference at each point. The same thing is true of the radius of the cylindrical wavefronts that would be generated by a fluorescent tube. Although the wavefronts produced by a point source are always concentric spheres in principle, when the source is very far away the radii of the spheres are so large that they look like plane waves to an observer (Just as the Earth looks flat when viewed from a point near its surface.).

There is a remarkable relation between the wavefronts corresponding to different values of $t$. This relation was discovered in 1678 by the great Dutch physicist Christian Huygens (1629-1695) who was a contemporary of Newton and wrote a treatise called "Traite de la Lumiere"on the wave theory of light. Huygens' principle describes how a wavefront moves in space. Although Huygens' principle was initially stated without any proof, a slightly modified form of it was later (about 1815) derived by Fresnel from the mathematical theory of waves. We state this principle as the following theorem which its proof is given in [Arn13].

Huygens' Theorem. [Arn13] Let $\phi_{p}(t)$ be the wavefront of the point $p$ after time $t$. For every point $q$ of this wavefront, consider the wavefront after time s, i.e. $\phi_{q}(s)$. Then the wavefront of the point $p$ after time $s+t, \phi_{p}(s+t)$, will be the envelope of the wavefronts $\phi_{q}(s)$, for $q \in \phi_{p}(t)$.

Actually the principle states that

1. every point on the wavefront of a propagating wave (the primary wave) is the source of secondary spherical wavefronts; and

2. the envelope of the wavefronts of all those secondary waves is the wavefront of the primary wave.

A key point is that all those waves propagate at the same velocity. This is clearer on a drawing. See the picture 4.3 and note that the wavefront emanating from $q$ is in fact an spherical wavefronts. In this theorem the point $p$ could be replaced by a curve, surface, or in general by a closed set. Also we have this theorem on any smooth manifold and the propagation of light can be replaced by the 


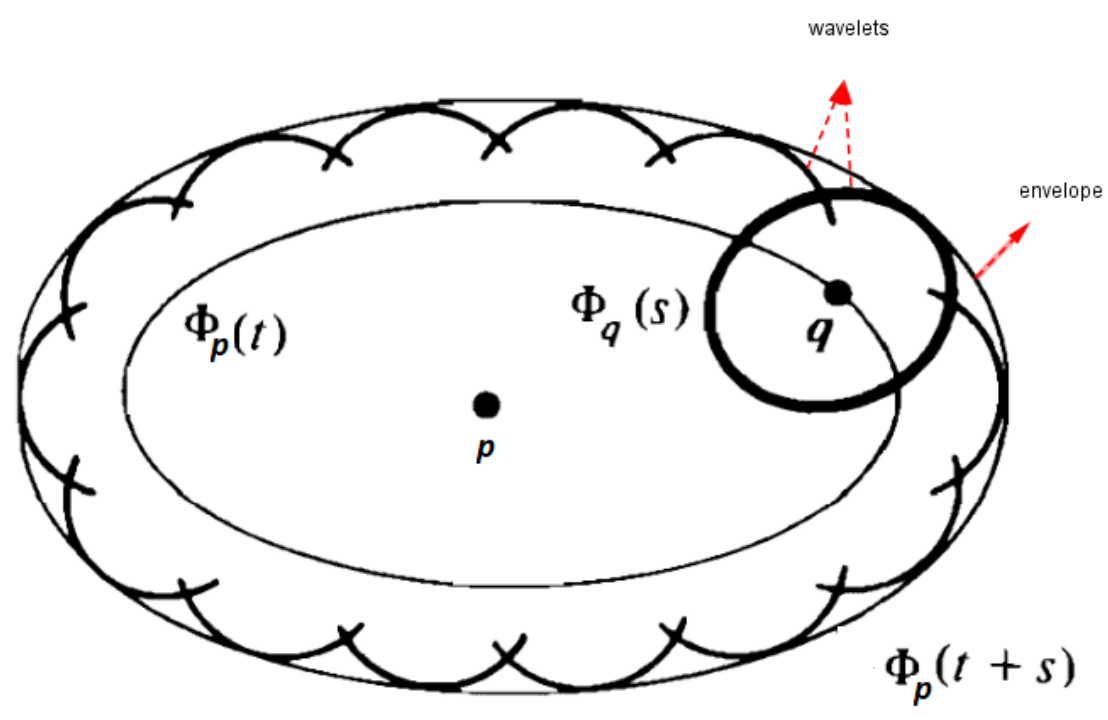

Figure 4.3: Envelope of wavefronts [Arn13]

propagation of any disturbance transmitting itself locally. Before continue with the part to which we are interested more, we explain some of the physical concepts a bit more. ${ }^{1}$

Note that when a wave travels in a single medium at a constant speed, the Huygens' construction preserves the general form of the wavefront. That is, spheres propagate and become larger spheres, cylinders become larger cylinders, etc. If a portion of the wavefront enters a different medium (enters glass from air, for example), then the wavefronts generated by each portion of the wavefront travel with the velocity that is appropriate for the medium that the wavefront is in. That is, the wavefronts in the medium where the speed of light is less will have smaller radii than the wavefronts in the original medium.

\subsubsection{The translation of Huygens' principle into the language of mathematics}

Provided that the leaves of a given foliation satisfy in the principle, Huygens' principle says that given a leaf $L$ of the foliation, one can consider the indicatrices centered at the points of $L$ and then draw the hypersurface that is tangent to each indicatrix (in a single point). This hypersurface is another leaf of the foliation. Following this methodology one can predict the behavior of a propagation (foliation).

\subsection{A mathematical modeling for wildfire}

In this part we give a mathematical paradigm of the fire spread in a submanifold of a manifold $M$. Two type of fire fields are verified. First a fire filed of grass which is uniform and the fire starts at time 0 from some area $A$ and we study its spread from time 0 to some time $t$. The other field of fire that we are interested in is the field which contains some uniform fuel such as oil in which the fire starts from an area $A$ at time 0 and the fuels burns for a while. In both these two cases we study the spread according to the area $A$ as an reference. Note that this area $A$ might be a point, a wavefront or some subset. Observe that the difference in these two cases is that in the first case

\footnotetext{
${ }^{1}$ Some parts of this text is based on notes of Professor Judah Levine at University of Colorado Boulder (www.colorado.edu/physics/phys $1230 /$ phys $1230_{\text {f }}$ a01/topic 14. html).
} 
the fire starts from $A$ at time 0 and as time passes the wavefronts get distance from $A$ and the particles of fire do not return toward the initial wavefront. However in the second case the fire starts at time 0 from $A$ and spreads in such a way that first the wavefronts get distance from the initial wavefront and then we have some new wavefronts that get closer to $A$. Indeed in this case as long as we have the fuel, some of the particles of fire move toward $A$ and some of them toward the opposite direction. In order to study these two phenomena we shall restate the Huygens's principle in the language of fire. As Anderson and et al already in [ACDMP82] gave a reasonable interpretation we just mention what they said in their work. Consider an arbitrary wavefront at time $t_{0}$ (Figure 4.4). Imagine the fire to be suddenly extinguished and at the same time a new fire lit at one point $p$ of

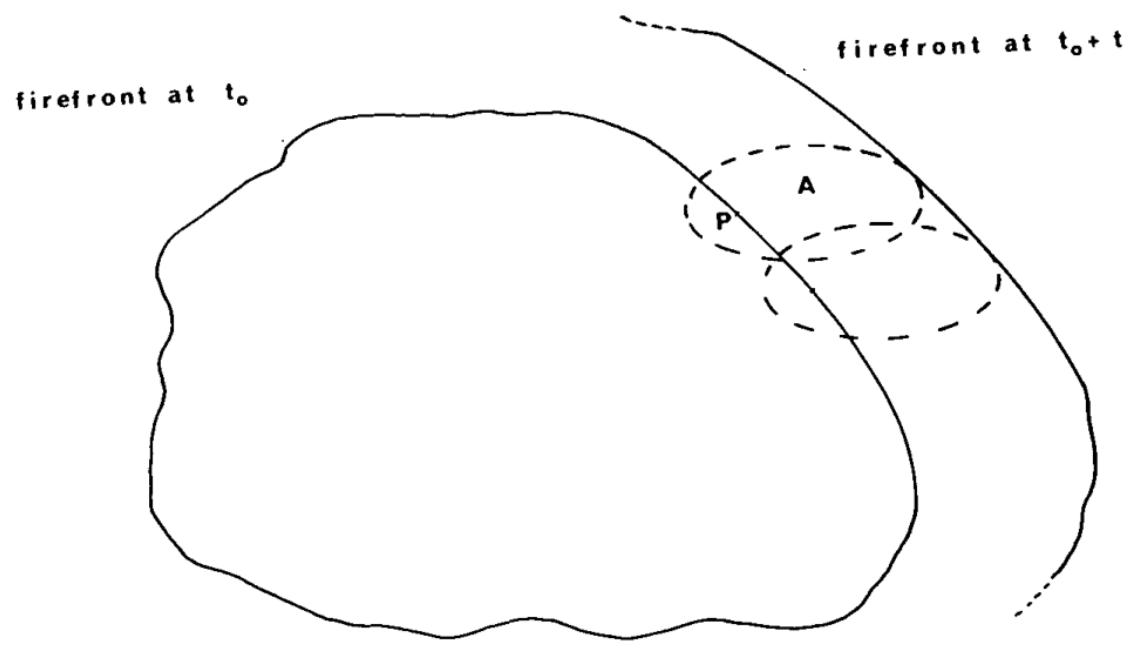

Figure 4.4: Huygens' principle applied to wavefronts

the wavefront. This new fire is left to burn for time $t$, as in Figure 4.4, forming a new wavefront. If conditions are uniform during this time from $t_{0}$ to $t_{0}+t$ then the new wavefront (firelet) will grow spherically into the unburnt region by the previous section. Consider now the region $A$ burnt out by this new wavefront (firelet). As the point $p$ moves around the original wavefront, the region $A$ will sweep out a large region around the original wavefront. Our application of Huygens' principle states that this region is in fact the region that would be burnt out by the original wavefront in the time $t_{0}$ to $t_{0}+t$. Equivalently, the wavefront at time $t_{0}+t$ is the envelope of the new sphere around $p$ as the point $p$ moves along the time $t_{0}$ front. This approach is illustrated in Figure 4.4. Huygens' principle gives us a method of predicting the wavefront at a future time if we know its present position.

Let's continue with the results. Through this subsection we assume that $M$ is a smooth manifold on some part of which a fire is spreading and $U \subset M$ is the domain burnt out by the fire particles during time from $t=0$ to $t=r$ which is also a smooth manifold. The manifold $M$ is equipped with a Finsler metric $F$ which is given by the indicatrices at points $p \in M$.

Proposition 4.3.1. Let $\rho: M \longrightarrow \mathbb{R}, \rho(p)=d_{F}(A, p)$ where $A$ is a compact subset of $M$ and $\rho(U)=[s, r]$, where $0<s<r$. Suppose that we do not have any cut loci in $\rho^{-1}(s, r)$. Then each $\rho^{-1}(t)$, for $t \in[s, r]$, is contained in a future cylinder at time $t-s$.

Proof. Let $\rho:=\rho_{+}$and observe that by Remark 1.4.17, $\left.\rho\right|_{U}$ is smooth. According to Lemma 1.4.18, $\rho$ is a transnormal function with $\mathfrak{b}=1$. Using Theorem 3.2.1 one has that, for every $t>s$ and 
$q \in \rho^{-1}(t)$

$$
d_{F}\left(\rho^{-1}(s), q\right)=d_{F}\left(\rho^{-1}(s), \rho^{-1}(t)\right)=\int_{s}^{t} d t=t-s
$$

which means each fire particle after time $t-s$, reaches to $\rho^{-1}(t)$ and so according to Definition 4.2.1, $\rho^{-1}(t)$ is the wavefront. Now let us verify the Huygens' principle. Assume that $e(\delta)$ is the forward envelope of radius $\delta$ of the wavefront $\rho^{-1}\left(t_{0}\right)$, for some $t_{0}$. Then according to Definition 4.2.2 for every $p \in e(\delta)$,

$$
d_{F}\left(\rho^{-1}\left(t_{0}\right), p\right)=d_{F}\left(\rho^{-1}\left(t_{0}\right), e(\delta)\right)=\delta .
$$

Observe that as $p \in e(\delta)$, there exists a path from some point $p_{0} \in \rho^{-1}\left(t_{0}\right)$ to the point $p$ along which the time of travel of a particle of the fire equals $\delta$. As $\rho^{-1}\left(t_{0}\right)$ is the wavefront, this particle emanates from some point in $\rho^{-1}(s)$ and reaches to point $p_{0}$ at time $t_{0}$ and as $p \in e(\delta)$, this particle reaches to point $p$ after $\delta$ seconds. So

$$
d_{F}\left(\rho^{-1}(s), p\right) \leq t_{0}+\delta
$$

Suppose that $d_{F}\left(\rho^{-1}(s), p\right)<t_{0}+\delta$. It means there exists a path from $\rho^{-1}(s)$ to $p$ through which a particle of fire travels in a time shorter than $t_{0}+\delta$. As

$$
d_{F}\left(\rho^{-1}(s), \rho^{-1}\left(t_{0}\right)\right)=t_{0}
$$

this particle meets $\rho^{-1}\left(t_{0}\right)$ at exactly time $t_{0}$. So the only possibility for the non-equality is when this particle reaches from $\rho^{-1}\left(t_{0}\right)$ to $p$ at time less than $\delta$ which is a contradiction with the fact that $d_{F}\left(\rho^{-1}\left(t_{0}\right), p\right)=\delta$. As a result,

$$
d_{F}\left(\rho^{-1}(s), p\right)=t_{0}+\delta:=t
$$

which means $p$ belong to the wavefront $\rho^{-1}(t)$ and so we have $e(\delta) \subset \rho^{-1}(t)$. Now let's prove the other relation that is $\rho^{-1}(t) \subset e(\delta)$. Assume that $p \in \rho^{-1}(t)$. Using Theorem 3.2.1, one has

$$
d_{F}\left(\rho^{-1}\left(t_{0}\right), p\right)=t-t_{0}=\delta
$$

and consequently $p \in e(\delta)$.

To terminate the proof, observe that each particle of fire emanates from a point belong to $f^{-1}(s)$ and reaches to $f^{-1}(c)$ in the shortest time. Hence with respect to the definition of the geodesics in Finsler spaces the track of each fire particle coincides with the track of a a geodesic of $F$. Now assume that $\alpha$ is the unit speed geodesic such that

$$
d_{F}\left(\rho^{-1}(s), \rho^{-1}(t)\right)=d_{F}\left(\rho^{-1}(s), p\right)=L\left(\left.\alpha\right|_{[0, t]}\right)=t .
$$

According to Theorem 3.2.1, $\left.\alpha\right|_{[0, r]}$ is an extension of the integral curve of $\nabla \rho$. So $\left.\alpha\right|_{(0, r)}$ is the integral curve of $\nabla \rho$ and by Lemma 1.3 .32 it is orthogonal to each $\rho^{-1}(t)$. As we have this fact for every $t \in(0, r)$, by taking a limit one can get the desires assertion.

proposition 4.3.1 is useful when one wants to model the fire spreading in, for example, a field of grass. Now consider the case in which the field of fire is, for example, a fuel and the fire already has 
started and in some time $r$ we start verifying whose spread considering $A$ as the reference. We are interested in modeling the behavior of the particles at an interval of time $[-r,-s]$. Here by time $-r$ we mean $r$ seconds before the particles reach to $A$. One can have the similar result as Proposition 4.3.1 in which $\rho_{-}$is replaced with $\rho_{+}$as follows.

Lemma 4.3.2. Define $\rho_{-}: M \longrightarrow \mathbb{R}, \rho_{-}(p)=-d_{F}(p, A)$ where $A$ is a compact subset of $M$ and let $\rho_{-}(U)=[-r,-s]$. Suppose that we do not have any cut loci in $\rho_{-}^{-1}(-r,-s)$. Then each $\rho_{-}^{-1}(t)$, for $t \in[-r,-s]$, is the wavefront at time $t$ and the Huygens' principle is satisfied by

$$
\left\{\rho_{-}^{-1}(t)\right\}_{t \in[-r,-s]}
$$

Furthermore, the track of each fire particle is the geodesic of $F$ and it is orthogonal to each wavefront $\rho_{-}^{-1}(t)$ at time $t$.

The proof can be done by the similar way as the mentioned Proposition (4.3.1) mutatis mutandis.

Example 4.3.3. As a simple example let us recall Example 2.3.1. That is consider $\mathbb{R}^{2}$ while we have a wind $W=\left(0, \frac{1}{2}\right)$ with the associated Randers metric F. Indeed we have a fire spreading in a fuel field in $\mathbb{R}^{2}$ meanwhile a wind $W$ is blowing. Now consider the partitions given by 3.2.2 which are

$$
\mathcal{F}=\left\{\rho_{+}^{-1}(r)\right\}_{r \geq 0} \text { and } \mathcal{G}=\left\{\rho_{-}^{-1}(s)\right\}_{s \leq 0} .
$$

Then we are faced with two bunches of wavefronts, $\mathcal{F}=\left\{\rho_{+}^{-1}(r)\right\}_{r \geq 0}$ which is associated to the fire particles that are getting far from the origin and $\mathcal{G}=\left\{\rho_{-}^{-1}(s)\right\}_{s \leq 0}$ that is associated to the fire particles that are traveling toward the origin. As it can be seen in Picture 3.1 these two partitions are quite disjoint and do not coincide. Now consider the geodesics $\alpha(t)=\frac{p}{\|p\|} t$, where $p \in \mathbb{R}^{2}$ and $t>0$, in $\mathbb{R}^{2}$ with the Euclidean metric. Then according to Lemma 2.2.14

$$
\gamma(t)=\varphi(t, \alpha(t))=\left(0, \frac{t}{2}\right)+\frac{p}{\|p\|} t
$$

is the associated F-geodesic where $\varphi(t, p)=\left(0, \frac{t}{2}\right)+p$ is the flow of $W$. Note that according to Corollary 2.2.7, as $\alpha^{\prime}(t)$ is in the horizontal space with respect to the Euclidean metric then $\gamma^{\prime}(t)$ is orthogonal to each wavefront and also it meets all of the wavefronts given by $\mathcal{F}$ (as it is expected with respect to Proposition 4.3.1). One can say the same things about the geodesics $\tilde{\alpha}(t)=-\frac{p}{\|p\|} t$ and whose associated geodesics

$$
\tilde{\gamma}=\left(0, \frac{t}{2}\right)-\frac{p}{\|p\|} t
$$

That is $\tilde{\gamma}$ is orthogonal to all the wavefronts $\mathcal{G}$ and it intersects all.

Remark 4.3.4. Let $\rho_{+}(U)=[a, b]$ and there are just finite many critical values $r_{1}, \ldots, r_{m}$ for $\rho_{+}$at $[a, b]$ such that the critical sets are disjoint subsets. Then Proposition 4.3 .1 is valid for each interval $\left[r_{i}, r_{i+1}\right]$, for $i=1, \ldots, m-1$, provided that $\rho_{+}^{-1}\left[r_{i}, r_{i+1}\right]$ is a smooth submanifold of $M$. The same result is valid for $\rho_{-}$.

Corollary 4.3.5. For the wavefront $\rho_{+}^{-1}(t)$ one can write

$$
\rho_{+}^{-1}(t) \subset\left\{\exp _{p}(t v) \mid p \in \rho_{+}^{-1}(0) \text { and } v \perp \rho_{+}^{-1}(0) \text { with } F(v)=1\right\} \text {. }
$$


Proof. According to Remarks 3.2.11 and 1.4.24 the proof is at hand.

\subsubsection{A special case}

As it was mentioned in Chapter 1, there are some methods to find the Finsler metric using the equation of the indicatrix in the tangent space. Let us give a method in the special case of a Randers space $(M, F)$, where $M$ is of dimension 2, which has been discussed by [Mar16]. Assume that we are given an indicatrix field $\Sigma(p)=E_{(u, v)}$ at the tangent spaces $T_{p} M$ for $p \in M$. As it was mentioned in Subsection 1.2.2, in Riemannian geometry the indicatrix is a quadratic surface with coefficients equal to the fundamental tensor $h_{i j}$ which is in our case a rotated ellipse. Also by Lemma 2.2.4, the indicatrix in the associated Randers space is nothing but a translation of the Riemannian indicatrix by the wind. So at the tangent space $\left.T_{p} M, E_{(} u, v\right)$ can be parametrized as following

$$
E_{(u, v)}=\operatorname{Rot}(\theta(u, v)) \cdot\left(\left[\begin{array}{l}
a(u, v) \cos \beta \\
b(u, v) \sin \beta
\end{array}\right]+\left[\begin{array}{l}
w_{1}(u, v) \\
w_{2}(u, v)
\end{array}\right]\right)
$$

where

$$
\operatorname{Rot}(\theta(u, v))=\left[\begin{array}{rr}
\cos \theta(u, v) & \sin \theta(u, v) \\
-\sin \theta(u, v) & \cos \theta(u, v)
\end{array}\right]
$$

which is a rotation in the clock-wise direction and $W=\left(w_{1}, w_{2}\right)$ is the wind which is sufficiently small such that the origin of the tangent plane is still contained in the indicatrix. Note that this indicatrix field, as it was mentioned above, in the associated Riemannian space $(M, h)$ can be simply written as

$$
\left[\begin{array}{l}
x(\beta) \\
y(\beta)
\end{array}\right]=\operatorname{Rot}(\theta(u, v)) \cdot\left[\begin{array}{l}
a(u, v) \cos \beta \\
b(u, v) \sin \beta
\end{array}\right] .
$$

Let us find the classical quadratic equation $Q(x, y)=1$ associated to this parametrization. From EQ. 4.3 one gets

$$
\operatorname{Rot}^{-1}(\theta(u, v)) \cdot\left[\begin{array}{l}
x(\beta) \\
y(\beta)
\end{array}\right]=\left[\begin{array}{l}
a(u, v) \cos \beta \\
b(u, v) \sin \beta
\end{array}\right] .
$$

which gives

$$
\cos \beta=\frac{x \cos \theta(u, v)-y \sin \theta(u, v)}{a(u, v)} \quad \text { and } \quad \sin \beta=\frac{x \sin \theta(u, v)+y \cos \theta(u, v)}{b(u, v)} .
$$

So

$$
\begin{aligned}
1 & =\cos ^{2} \beta+\sin ^{2} \beta \\
& =\left(\frac{x \cos \theta(u, v)-y \sin \theta(u, v)}{a(u, v)}\right)^{2}+\left(\frac{x \sin \theta(u, v)+y \cos \theta(u, v)}{b(u, v)}\right)^{2} \\
& =Q(x, y) .
\end{aligned}
$$

This equation can also be written as

$$
\left[\begin{array}{ll}
x & y
\end{array}\right] h(u, v)\left[\begin{array}{l}
x \\
y
\end{array}\right]=1
$$


where

$$
h(u, v)=\frac{1}{2} \operatorname{Hess} Q(x, y)=\left[\begin{array}{cc}
\frac{\cos ^{2} \theta}{a^{2}}+\frac{\sin ^{2} \theta}{b^{2}} & \frac{-\cos \theta \sin \theta}{a^{2}}+\frac{\sin \theta \cos \theta}{b^{2}} \\
\frac{-\cos \theta \sin \theta}{a^{2}}+\frac{\sin \theta \cos \theta}{b^{2}} & \frac{\cos ^{2} \theta}{b^{2}}+\frac{\sin ^{2} \theta}{a^{2}}
\end{array}\right]
$$

that is the Riemannian metric $h$ on $M$. Comparing with Definition 1.2 .13 , it can be seen that this relation is completely reasonable. Now that we could have found the Riemannian metric $h$, using Proposition 2.2.2, the Randers metric $F$ is obtained just by some replacements.

Now suppose that we are given a fire igniting from a point on the plane. We can consider this point as the origin and assume that the fire ignites from the origin $(0,0) \in \mathbb{R}^{2}$. The goal is to predict the propagation of the fire having the following information about the indicatrix $\Sigma^{h}$, where $h$ is a Riemannian metric on $\mathbb{R}^{2}$, and the wind $W$.

$$
a(u, v)=\frac{1}{2}, \quad b(u, v)=\frac{3}{2}, \quad W(u, v)=\left(\frac{1}{4}, \frac{1}{2}\right), \quad \theta(u, v)=v-1 .
$$

According to EQ. 4.2, the corresponding indicatrix $\Sigma^{F}$ (ellipse) is given by the following formula:

$$
\left\{\begin{array}{l}
x=\cos (v-1)\left(\frac{1}{2} \cos \beta+\frac{1}{4}\right)+\sin (v-1)\left(\frac{3}{2} \sin \beta+\frac{1}{2}\right) \\
y=-\sin (v-1)\left(\frac{1}{2} \cos \beta+\frac{1}{4}\right)+\cos (v-1)\left(\frac{3}{2} \sin \beta+\frac{1}{2}\right),
\end{array}\right.
$$

where $\beta \in[0,2 \pi]$. By Proposition 4.3.1, the propagation of the fire satisfies in the Huygens's principle. That is one needs to first find $\Sigma^{F}$, that is nothing but a translation of $\Sigma^{h}$ by $W$, and then find $\Sigma_{2}^{F}$, using the indicatrices $\Sigma^{F}(q)$ centered at points $q$ of $\Sigma^{F}$, and so on. Here in Picture 4.5, in the left hand picture the indicatrix $\Sigma^{F}$ is determined with blue color and then the indicatrices $\Sigma^{F}(q)$, where $q \in \Sigma^{F}$, with pink. In the right hand picture, as well as these indicatrices, the fire location at time $t=2$, that is the curve closed to the indicatrices, is determined with the light blue color. In Picture 4.6, in the left hand picture the locations of fire at times $t=1$ and $t=2$, and in the right hand picture, its locations at times $t=1, t=2$, and $t=3$ are determined.

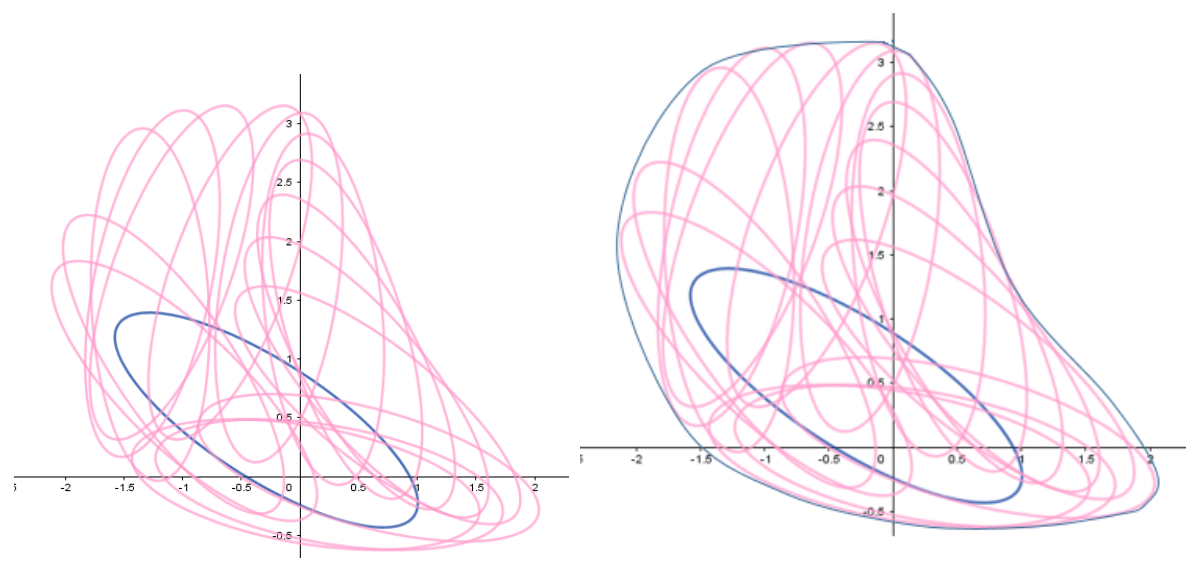

Figure 4.5: the indicatrices

\section{4 the propagation of water waves}

Huygens' principle works for all types of waves, including water waves, sound waves, and light waves. We will use it to show that the propagation of waves in water, under some conditions, in any 

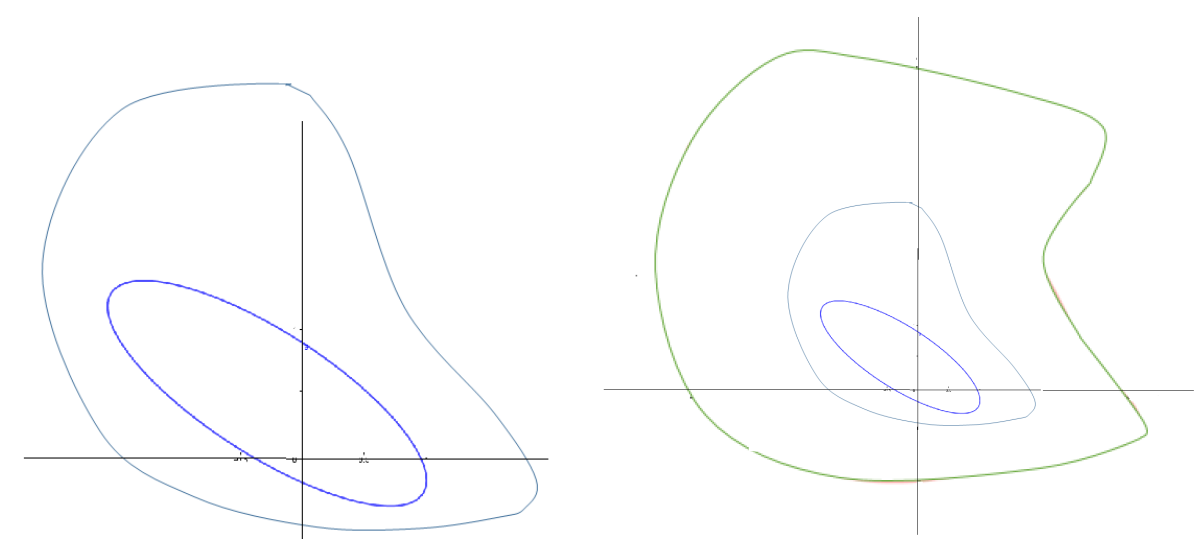

Figure 4.6: propagation of fire

dimensions coincides with the foliation related to some smooth function $f$, as we discussed it for the fire spread. Observe that, generally, the Huygens' principle is valid only in an odd dimensional space. To see what it means let's see a case. Drop a stone in a calm pond. A circular wave emanates

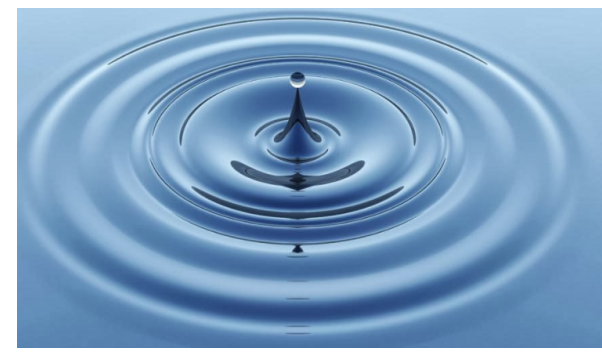

Figure 4.7: dropping a stone in a pond

outward. If the principle was valid for all dimensions, the surface of the water must stay perfectly calm after the wave passes, while the surface of the pond keeps differing slightly from its static. The same thing happens in any even dimensional space. Of course, the leading edge of a wave always propagates at the same speed, regardless of the dimension of the space. To be more accurate, Huygens' principle is more significant for what it says about what happens behind the leading edge of the disturbance, which is not our case in this work, indeed. Essentially it just says that all the phases propagate at the same speed and this is exactly what we need in this work. Now let's see how to obtain the wavefronts using the Huygens' principle. First note that, the waves have moving crests (or peaks) and troughs. A crest is the highest point the medium rises to and a trough is the lowest point the medium sinks to. Crests and troughs on a transverse wave are shown in Figure 4.8. Assume that we have the wavefront at some time $t_{0}$. We put the crest wavefronts and the trough

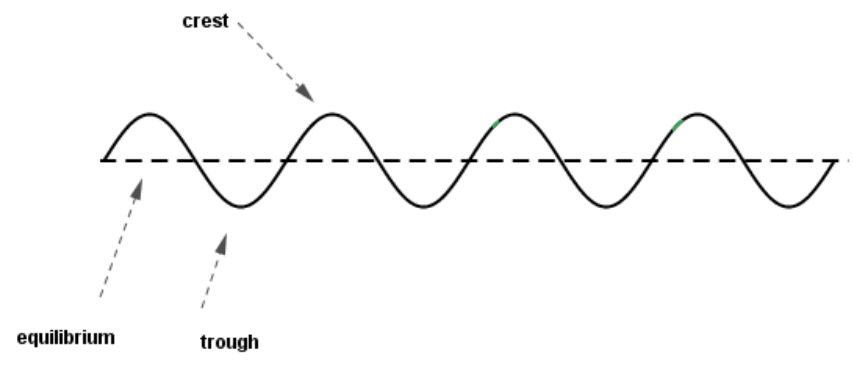

Figure 4.8: crest and drop 
wavefronts on it. More wavefronts we have, more easier we will be able to locate the next wavefront. See picture 4.9, where a plane wavefront with both the crest and trough wavefronts on it are shown. Now the surface tangent to the wavefronts is the wavefront. Observe that by the wavefront we

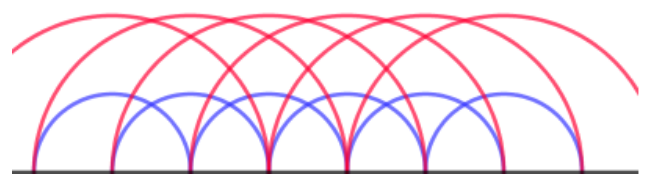

Figure 4.9: crest and trough wavefronts

mean both crest and trough wavefronts. See picture 4.10. In this case we have a proposition similar

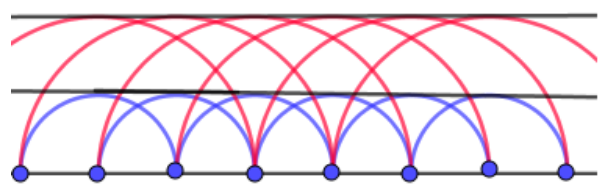

Figure 4.10: wavefront

to Proposition 4.3.1 in which the same proof is applicable, mutatis mutandis. Assume that $M$ is a smooth manifold which is also some domain of water. The manifold $M$ is equipped with some Finsler metric $F$.

Proposition 4.4.1. Given a compact subset $A$ of Finsler manifold $M$, define $\rho_{+}: M \backslash A \longrightarrow \mathbb{R}$ as $\rho_{+}(p)=d_{F}(A, p)$ and let $\rho_{+}(M \backslash A)=(0, r]$, where $0<r$. Suppose that a spread of water waves has started with the wavefront at time $t=0$ equals to $\partial A$ and also assume that we do not have any cut loci in $\rho_{+}^{-1}(0, r)$. Then each $\rho_{+}^{-1}(t)$, for $t \in(0, r]$, is the wavefront at time $t$ and the Huygens' principle is satisfied by the wavefronts

$$
\left\{\rho_{+}^{-1}(t)\right\}_{t \in(0, r]} \text {. }
$$

Furthermore, the track of each water molecule is the geodesic of $F$ and also it is orthogonal to all wavefronts $\rho_{+}^{-1}(t)$ at time $t$.

Let us rewrite Proposition 4.4.1 for the case in which a stone drops in a $n$-dimensional pond. We show this pond with $M$ which is also a Finsler space and $A$ is the spot where the stone enters the water. We also assume that there is not any other forces that disturb the water and the waves are spreading regularly. That is we do not have any cut locus. One has the following corollary.

Corollary 4.4.2. Consider $\rho_{+}: M \backslash A \longrightarrow \mathbb{R}, \rho_{+}(p)=d_{F}(A, p)$ and let $\rho_{+}(M \backslash A)=(0, r]$, where $0<r$. Then, for $t \in(0, r]$, each $\rho_{+}^{-1}(t)$ is the geometrical sphere that coincides with the wavefront at time $t$. Moreover the track of each molecule of water coincides with a radius of this sphere. See Picture 4.11.

Example 4.4.3. Let us recall Example 2.4.8. Assume that $D$ is a source of water and $W$ is the wind. Consider the small circles of radius less than 1 around the origin and observe that because the wind is tangent to each such circles, by Lemma 2.2.4, the indicatrices associated to $F$ coincide 


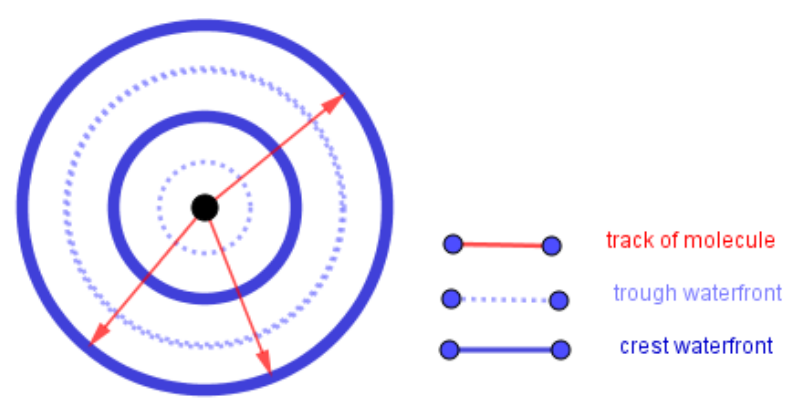

Figure 4.11: propagation of water in pond

with these circles. Now consider the function $f: D \longrightarrow \mathbb{R}, f(x, y)=x^{2}+y^{2}$. One can say that the preimage of each values $r \in[0,1)$ coincides with a circle of radius less than 1 . As $W$ is tangent to the leaves, according to Lemma 2.2.10, we have that

$$
F(\nabla f)=\|\bar{\nabla} f\|
$$

where $\bar{\nabla} f$ and $\nabla f$ are the gradients of $f$, resp., with respect to the metrics $h$ and $F$. Here $h$ is the canonical Euclidean metric on $\mathbb{R}^{2}$. According to this discussion, $F^{2}(\nabla f)=2 f$ that is $f$ is $F$ transnormal. According to Proposition 4.4.1, the circles satisfy the Huygens' principle and also the track of each molecule of water is a geodesic emanating from the origin and meets all of the leaves orthogonally. In picture 4.12 the geodesic

$$
\gamma(t)=\frac{\sqrt{2} t}{2}(\cos t-\sin t, \sin t+\cos t)
$$

with some of the leaves are shown.

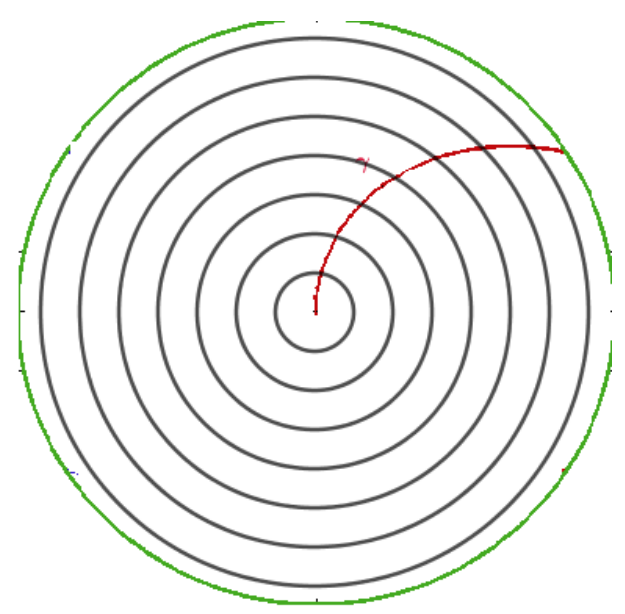

Figure 4.12: The open disc $D$

Here one can have the same result as Proposition 4.3.1 for the case of water and each Ftransnormal function $f: M \longrightarrow \mathbb{R}$ and also an application of Theorem 3.3.1 on the propagation of water waves as following:

Proposition 4.4.4. Suppose that $M$ is a source of water and $f:(M, F) \longrightarrow \mathbb{R}$ is a $F$-transnormal function with $F^{2}(\nabla f)=\mathfrak{b}(f)$ and $f(M)=[a, b]$. Assuming that we have a propagation of water waves for which $f^{-1}(a)$ is the wavefront at time 0 , then 
a) for every $c \in[a, b], f^{-1}(c)$ is the wavefront at time $r_{a, c}=\int_{a}^{c} \frac{d s}{\sqrt{\mathfrak{b}(s)}}$,

b) $\left\{f^{-1}(c)\right\}_{c \in[a, b]}$ satisfies in Huygens' principle,

c) the track of each molecule of water is a geodesic of $F$ that joins $f^{-1}(a)$ to $f^{-1}(b)$ which is also orthogonal to each wavefront,

If moreover, $f$ is analytic, $M$ is compact, $a$ and $b$ are the only critical values, and $f^{-1}(a)$ and $f^{-1}(b)$ are of codimensions greater than 1, then one can consider $f^{-1}(b)$ as the wavefront at time 0 for the (return) propagation of the waves and say

d) for every $c \in[a, b], f^{-1}(c)$ is the wavefront at time $r_{c, b}=\int_{c}^{b} \frac{d s}{g_{\nabla f}(\nabla f, \xi)}$, where $\xi \in \mathfrak{C}^{-1}\left(f^{-1}(c)\right)$ which is on the opposite side of $\nabla f$ with respect to the hyperplane $T_{\pi(\xi)} f^{-1}(c)$,

e) $\left\{f^{-1}(c)\right\}_{c \in[a, b]}$ satisfies in Huygens' principle,

$f)$ the track of each molecule of water is a geodesic of $F$ that joins $f^{-1}(b)$ to $f^{-1}(a)$ which is also orthogonal to each wavefront.

Proof. The proof of the three first items is just the repetition of the proof of Proposition 4.3.1, mutatis mutandis. To prove the other items, first by Theorem 3.3.1 and the hypothesis, we have that for every $c, d \in[a, b], f^{-1}(d)$ is equidistant to $f^{-1}(c)$. Hence according to Proposition 3.5.10,

$$
d_{F}\left(q, f^{-1}(c)\right)=d_{F}\left(f^{-1}(b), f^{-1}(c)\right)=\int_{c}^{b} \frac{d s}{g_{\nabla f}(\nabla f, \xi)}:=r_{c, b},
$$

for every $q \in f^{-1}(b)$. This means that $f^{-1}(c)$ is the wavefront at time $r_{c, b}$. Now again by the same argument provided for Proposition 4.3.1, the proof can be done, mutatis mutandis. To prove item $(f)$, as each particle of fire emanates from a point belong to $f^{-1}(b)$ and reaches to $f^{-1}(c)$ in the shortest time, with respect to the definition of the geodesics in Finsler spaces the track of each fire particle coincides with the track of a a geodesic of $F$. Moreover by Theorem 3.3.1, this geodesic remains orthogonal to all of the level set (wavefront).

Example 4.4.5. As an example let us recall Example 3.3.12. In this example assume that $M=S^{n}$ is a source of water in which the wind $W$ is blowing on the surface of the water. As we saw in the mentioned example $\tilde{f}$ is a F-transnormal function and we are faces with a SFF. In particular the leaves $\tilde{f}^{-1}(c)$, for $c \in[-1,1]$, satisfy in the Huygens' principle. Moreover, let's also recall Example 2.4.6 in which some of the geodesics for such a space are given. Then all of these geodesics meet once all of the leaves from $\tilde{f}^{-1}(-1)$ to $\tilde{f}^{-1}(1)$ and once from $\tilde{f}^{-1}(1)$ to $\tilde{f}^{-1}(-1)$. In Picture 4.13, the geodesic

$$
\gamma(t)=\left(\sin t \cos \frac{t}{2}, \sin t \sin \frac{t}{2}, \cos t\right)
$$

with some of the leaves are shown. 


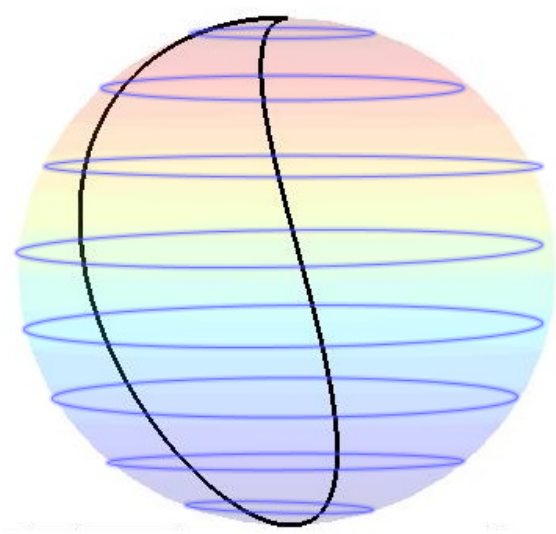

Figure 4.13 


\section{Chapter 5}

\section{some questions and suggestions for future works}

In the future we have plan to work on the following conjectures.

- Let $f: M \longrightarrow \mathbb{R}$ be $F$-transnormal and analytic with $f(M)=[a, b]$. Suppose that the level sets are connected. Then

- each regular level set is equifocal,

- the critical level sets $f^{-1}(a)$ and $f^{-1}(b)$ are embedded submanifolds,

$-\mathcal{F}=\left\{f^{-1}(c)\right\}_{c \in[a, b]}$ is a SFF.

- Let $(M, F)$ be a compact, connected, complete, and smooth Finsler manifold and $f: M \rightarrow$ $[a, b]$ be a smooth $F$-transnormal function with $F^{2}(\nabla f)=\mathfrak{b}(f)$, where $\mathfrak{b}$ is a $C^{1}$ function on $[a, b]$. Suppose that:

(a) the level sets are connected,

(b) $a$ and $b$ are the only singular values at $[a, b]$,

(c) $\mathfrak{b}^{\prime}(a) \neq 0 \neq \mathfrak{b}^{\prime}(b)$,

(d) the regular level sets are equidistant.

Then the singular level sets are submanifolds.

- Let $f:(M, F) \longrightarrow[a, b]$ be a $F$-transnormal function for which $\mathcal{F}=\left\{f^{-1}(c)\right\}_{c \in[a, b]}$ is a SFF. Then

$$
\cos \theta(f)=\frac{g_{\nabla f}(\nabla f, \xi)}{\sqrt{\mathfrak{b}(f) g_{\nabla f}(\xi, \xi)}},
$$

where $\xi \in \mathfrak{C}^{1}\left(f^{-1}(c)\right)$ which is on the opposite side of $\nabla f$ with respect to the hyperplane $T_{\pi(\xi)} f^{-1}(c)$ and $\theta=\varangle(\nabla f, \xi)$, for the regular value $c$.

In particular if the angle $\theta$ is not constant along each leaf, then the foliation cannot be Finslerian. 


\section{Appendix A}

\section{Pictures}

\section{A.0.1 Geodesics before and after the wind}

In following you may see the indicatrix and geodesic of example (2.3.1) at some points before and after the wind:
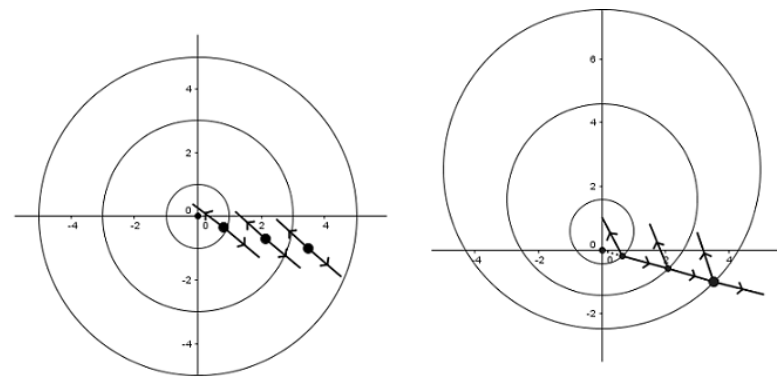

Figure A.1

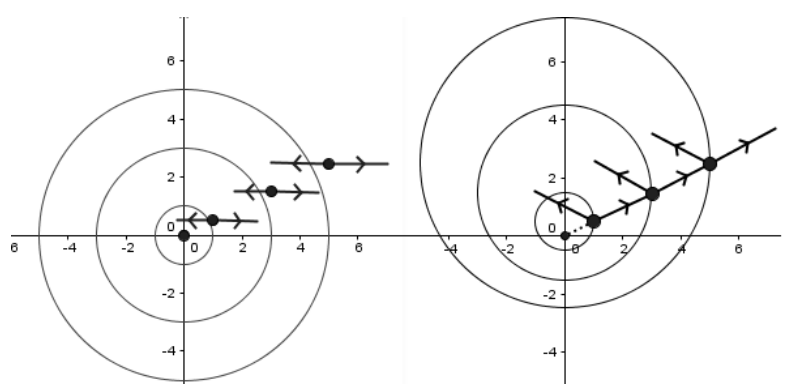

Figure A.2
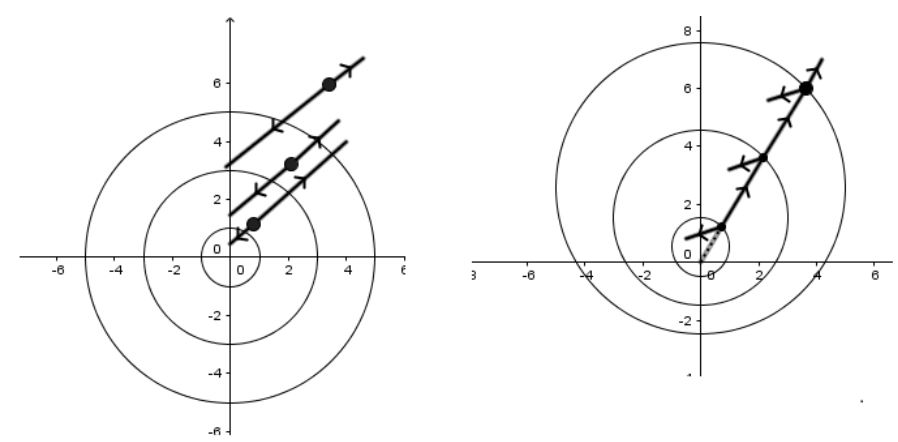

Figure A.3 


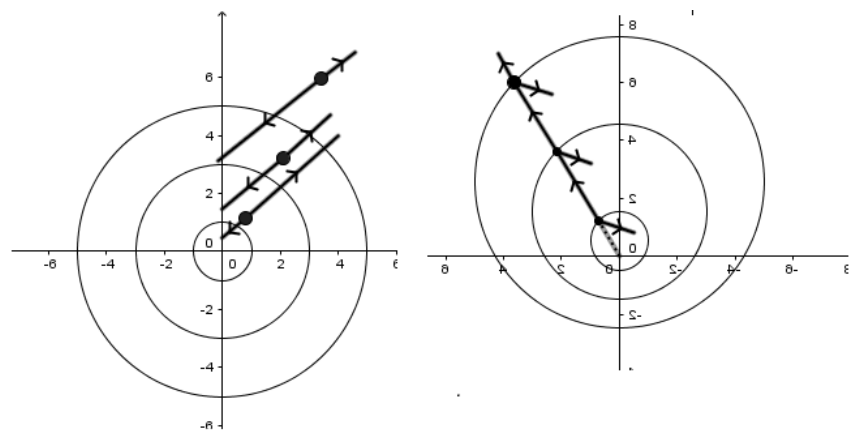

Figure A.4

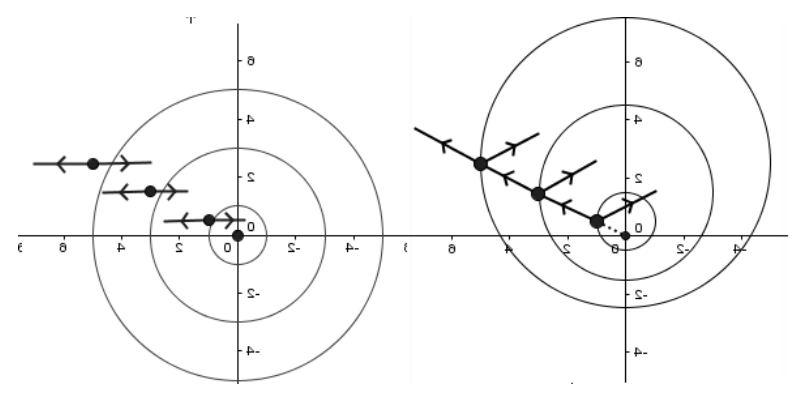

Figure A.5

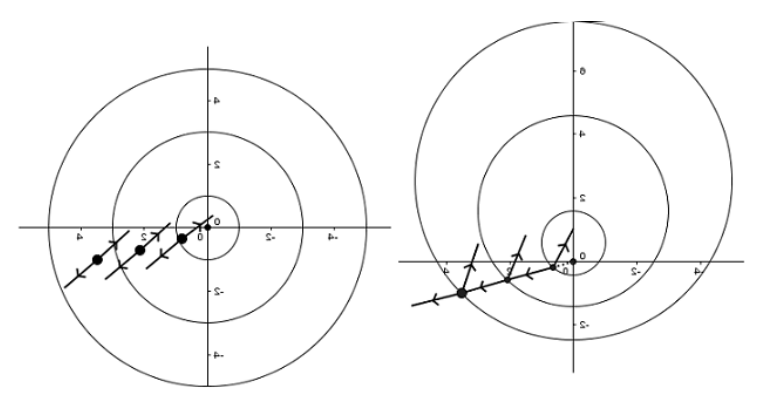

Figure A.6 


\section{Bibliography}

[AAJ17] Marcos M Alexandrino, Benigno O Alves e Miguel Angel Javaloyes. On singular finsler foliation. arXiv preprint arXiv:1708.05457, 2017. 82, 84

[ABS03] Peter L Antonelli, Andrej Bóna e Michael A Slawiński. Seismic rays as finsler geodesics. Nonlinear analysis: real world applications, 4(5):711-722, 2003. 4, 39

[ABT13] Marcos M Alexandrino, Rafael Briquet e Dirk Töben. Progress in the theory of singular riemannian foliations. Differential Geometry and its Applications, 31(2):248267, 2013. 1

[ACDMP82] DH Anderson, EA Catchpole, NJ De Mestre e T Parkes. Modelling the spread of grass fires. The ANZIAM Journal, 23(4):451-466, 1982. 105

[AIM13] Peter L Antonelli, Roman S Ingarden e Makoto Matsumoto. The theory of sprays and Finsler spaces with applications in physics and biology, volume 58. Springer Science \& Business Media, 2013. 5

[AJ11] Marcos M Alexandrino e Miguel Angel Javaloyes. On closed geodesics in the leaf space of singular riemannian foliations. Glasgow Mathematical Journal, 53(3):555-568, 2011. 26

[AJ17] Benigno Alves e Miguel Angel Javaloyes. A note on the existence of tubular neighbourhoods on finsler manifolds and minimization of orthogonal geodesics to a submanifold. arXiv preprint arXiv:1710.01699, 2017. 29

[Ale04] Marcos M Alexandrino. Integrable riemannian submersion with singularities. Geometriae Dedicata, 108(1):141-152, 2004. 1

[Ale06] Marcos M Alexandrino. Proofs of conjectures about singular riemannian foliations. Geometriae Dedicata, 119(1):219-234, 2006. 1

[Ale10] Marcos M Alexandrino. Desingularization of singular riemannian foliation. Geometriae Dedicata, 149(1):397-416, 2010. 1

[ÁPD01] J Álvarez Paiva e C Durán. Isometric submersions of finsler manifolds. Proceedings of the American Mathematical Society, 129(8):2409-2417, 2001. 25

[AR16a] Marcos M Alexandrino e Marco Radeschi. Closure of singular foliations: the proof of molino's conjecture. arXiv preprint arXiv:1608.03552, 2016. 1

[AR16b] Marcos M Alexandrino e Marco Radeschi. Mean curvature flow of singular riemannian foliations. The Journal of Geometric Analysis, 26(3):2204-2220, 2016. 1

[Arn13] Vladimir Igorevich Arnol'd. Mathematical methods of classical mechanics, volume 60. Springer Science \& Business Media, 2013. 101, 103, 104

[AT08] Marcos Alexandrino e Dirk Töben. Equifocality of a singular riemannian foliation. Proceedings of the American Mathematical Society, 136(9):3271-3280, 2008. 1 
[BCO16] Jurgen Berndt, Sergio Console e Carlos Enrique Olmos. Submanifolds and holonomy, volume 21. CRC Press, 2016. 25

[BCS12] David Bao, S-S Chern e Zhongmin Shen. An introduction to Riemann-Finsler geometry, volume 200. Springer Science \& Business Media, 2012. 1, 5, 8, 15, 18

[BH10] Augustin Banyaga e David Hurtubise. Morse-bott homology. Transactions of the American Mathematical Society, 362(8):3997-4043, 2010. 21

[BM07] Ioan Bucataru e Radu Miron. Finsler-Lagrange geometry: Applications to dynamical systems. Editura Academiei Romane Bucuresti, 2007. 4, 39

[BR04] David Bao e Colleen Robles. Ricci and flag curvatures in finsler geometry. A sampler of Riemann-Finsler geometry, 50:197-259, 2004. 5

$\left[\mathrm{BRS}^{+}\right.$04] David Bao, Colleen Robles, Zhongmin Shen et al. Zermelo navigation on riemannian manifolds. Journal of Differential Geometry, 66(3):377-435, 2004. 4, 39, 40

[CG12] M Cvetič e Gary W Gibbons. Graphene and the zermelo optical metric of the btz black hole. Annals of Physics, 327(11):2617-2626, 2012. 4, 39

[CJS14] Erasmo Caponio, Miguel Angel Javaloyes e Miguel Sánchez. Wind finslerian structures: from zermelo's navigation to the causality of spacetimes. arXiv preprint arXiv:1407.5494, 2014. 4, 39

[Got09] Srikanth Gottipati. Finsler optimal control theory. City University of New York, 2009. 4,39

[GW11] GW Gibbons e CM Warnick. The geometry of sound rays in a wind. Contemporary Physics, 52(3):197-209, 2011. 4, 39

[HYS16] Qun He, Songting Yin e Yibing Shen. Isoparametric hypersurfaces in minkowski spaces. Differential Geometry and its Applications, 47:133-158, 2016. 68

[Jav12] Miguel Angel Javaloyes. Conformally standard stationary spacetimes and fermat metrics. Em Recent Trends in Lorentzian Geometry, páginas 207-230. Springer, 2012. 4, 39

[Jav13] Miguel Angel Javaloyes. Chern connection of a pseudo-finsler metric as a family of affine connections. arXiv preprint arXiv:1303.6263, 2013. 6

[JS14] Miguel Angel Javaloyes e Bruno Learth Soares. Geodesics and jacobi fields of pseudofinsler manifolds. arXiv preprint arXiv:1401.8149, 2014. 1, 12, 15

[Kat73] Anatole B Katok. Ergodic perturbations of degenerate integrable hamiltonian systems. Mathematics of the USSR-Izvestiya, 7(3):535, 1973. 53

[Kri10] Alexandru Kristály. Economic Optimization Problems via Riemann-Finsler Geometry. Tese de Doutorado, Central European University, 2010. 4, 39

[Mar16] Steen Markvorsen. A finsler geodesic spray paradigm for wildfire spread modelling. Nonlinear Analysis: Real World Applications, 28:208-228, 2016. 4, 39, 108

[Miy13] Reiko Miyaoka. Transnormal functions on a riemannian manifold. Differential Geometry and its Applications, 31(1):130-139, 2013. 1

[PW12] Christian Pfeifer e Mattias NR Wohlfarth. Beyond the speed of light on finsler spacetimes. Physics Letters B, 712(3):284-288, 2012. 4, 39 
[QT15] Chao Qian e Zizhou Tang. Isoparametric functions on exotic spheres. Advances in Mathematics, 272:611-629, 2015. 21, 82, 87

[Ran41] Gunnar Randers. On an asymmetrical metric in the four-space of general relativity. Physical Review, 59(2):195, 1941. 7

[Rob07] Colleen Robles. Geodesics in randers spaces of constant curvature. Transactions of the American Mathematical Society, páginas 1633-1651, 2007. 1, 47, 53

[She01a] Zhongmin Shen. Finsler metrics with $\mathrm{k}=0$ and $\mathrm{s}=0$. arXiv preprint math $/ 0109060$, 2001. 39,47

[She01b] Zhongmin Shen. Lectures on Finsler geometry, volume 2001. World Scientific, 2001. $1,5,6,10,15,17,19,20,30$

[She13] Zhongmin Shen. Differential geometry of spray and Finsler spaces. Springer Science \& Business Media, 2013. 5

[Voi11] Nicoleta Voicu. On the fundamental equations of electromagnetism in finslerian spacetimes. Progress In Electromagnetics Research, 113:83-102, 2011. 4, 39

[Wan87] Qi-Ming Wang. Isoparametric functions on riemannian manifolds. i. Mathematische Annalen, 277(4):639-646, 1987. 1, 72

[Zil83] Wolfgang Ziller. Geometry of the katok examples. Ergodic Theory and Dynamical Systems, 3(1):135-157, 1983. 53, 54 